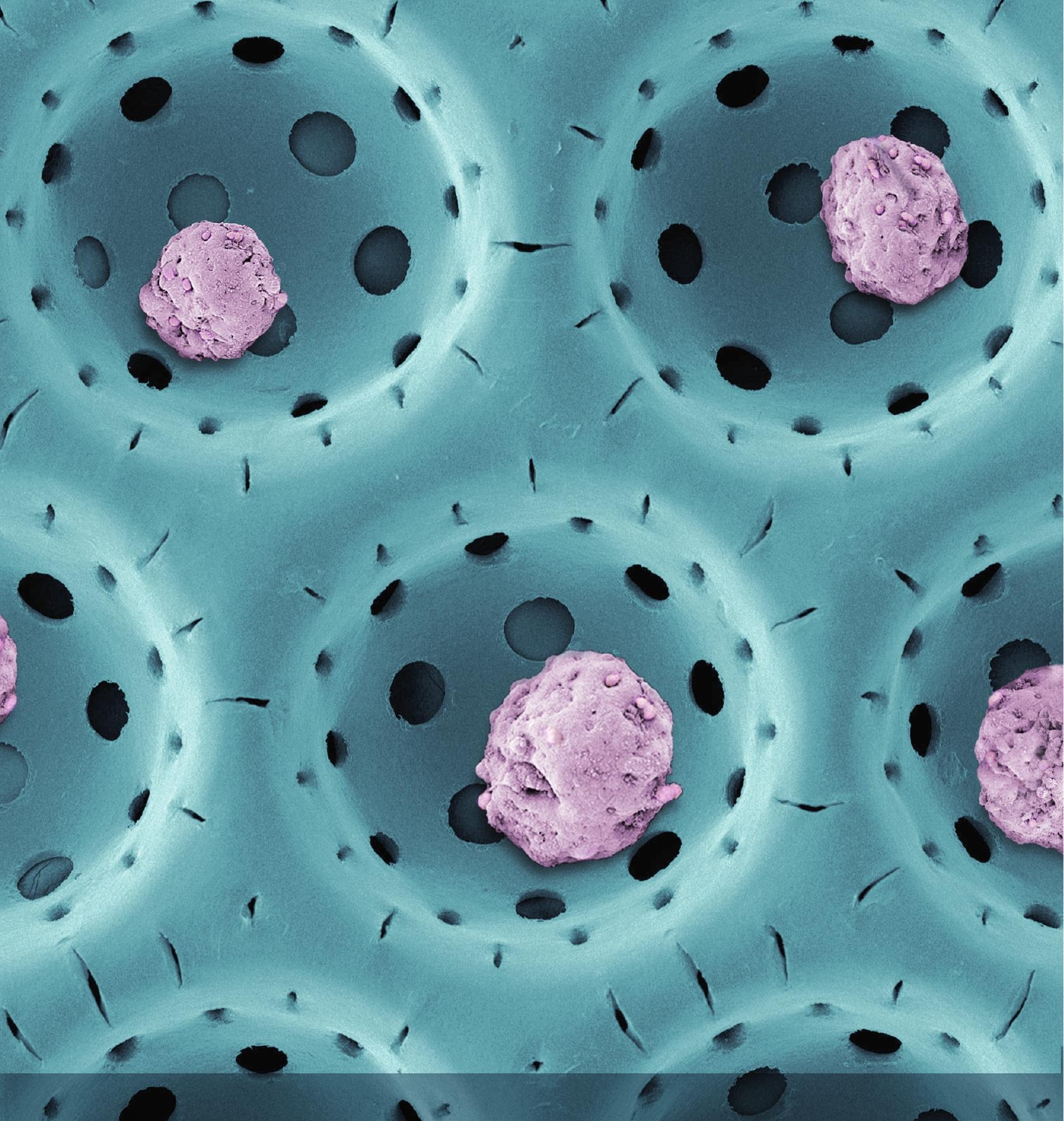

A Tissue Engineering Approach Towards Treatment of Type 1 Diabetes







\section{A Tissue Engineering Approach Towards Treatment of Type 1 Diabetes}

Elahe Hadavi

2018 


\section{Graduation Committee}

\section{Chairman}

prof. Dr.ir J.W. Hilgenkamp

University of Twente

\section{Promotor}

Prof. Dr. H.B.J. Karperien

University of Twente

\section{Co-promotor}

Dr. A.A. van Apeldoorn

University of Twente

\section{Members}

Prof. Dr. R.C.J.J. Passier

University of Twente

Prof. Dr. D. W. Grijpma

University of Twente

Prof. Dr. D. Stamatialis

University of Twente

Prof. Dr. P. de Vos

University Medical Center Groningen

Prof. Dr. R.A. Bank

University Medical Center Groningen

Dr. R. Truckenmüller

Maastricht University

\section{A Tissue Engineering Approach Towards Treatment of Type 1 Diabetes}

Elahe Hadavi, 2018

The work in this PhD thesis was performed in the department of Developmental BioEngineering (DBE) within the MIRA Institute for Biomedical Technology and Technical Medicine, and the Faculty of Science and Technology of the University of Twente, Enschede, The Netherlands.

The research was funded by the Dutch Diabetes Cell Therapy (DCTI) and the Dutch diabetes Research Foundation.

The printing of this thesis was supported by the Netherlands society for Biomaterials and Tissue Engineering (NBTE).

\section{MIRA UNIVERSITY

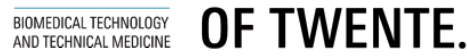



Cover atr: Ivan Lorenzo-Moldero and Elahe Hadavi Printed by Optima, Rotterdam, The Netherlands

(C) E. Hadavi, The Netherlands, 2018

ISBN: 978-94-6361-087-2



DOI: $10.3990 / 1.9789463610872$ 


\title{
A TISSUE ENGINEERING APPROACH TOWARDS TREATMENT OF TYPE 1 DIABETES
}

\section{DISSERTATION}

\author{
to obtain \\ the degree of doctor at the University of Twente, \\ on the authority of the rector magnificus, \\ prof. dr. T.T.M. Palstra \\ on account of the decision of the graduation committee, \\ to be publicly defended \\ on Thursday, March $29^{\text {th }} 2018$, at 12.45
}

by

\section{Elahe Hadavi}

Born on September $21^{\text {st }}, 1984$

in Ardebil, Iran 
The dissertation has been approved by:

Prof. Dr. H.B.J. Karperien (Supervisor)

Dr. A.A. van Apeldoorn (Co- Supervisor) 
"ان•

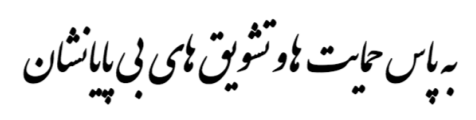

This thesis is dedicated to my beloved parents for their endless support and inspiration 



\section{Contents}

Chapter 1 General introduction

Chapter 2 Tissue engineering of islets for the treatment of type 1 diabetes

Chapter 3 Degradation kinetics and changes of Poly(ester-Urethane) and poly(ethylene glycol terephtalate-polybutylene terephthalate) thin films used for internal biomedical applications.

Chapter 4 Microcontact printing of extracellular matrix induces selfassembly of INS-1E cells in islet-like organoids with increased insulin secretion

Chapter 5 Mimicking the islet extracellular matrix niche in microwell islet delivery devices using collagen-IV and laminin 111 leads to improved insulin secretion in human islets

Chapter 6 PEOT-PBT and poly(ester urethane) microwell scaffolds for extrahepatic islet transplantation

Chapter 7 Endothelial growth factor delivery systems from assembled polymer nanolayers

Chapter 8 Conclusion and outlook

Summary/ Samenvatting

Acknowledgements

List of publications 

Chapter 1

General introduction 


\section{Diabetes type 1}

Type 1 diabetes is an autoimmune disease in which the immune system destroys the insulin producing $\beta$-cells of the pancreatic islets, which results in absolute insulin deficiency [1]. The global prevalence of diabetes was estimated to be 415 million people in 2015 and it is predicted to rise to 642 million in 2040. Based on the reports from high income countries, the prevalence of type 1 diabetes accounts for $7-12 \%$ of all people with diabetes $[1,2]$.

The most common method to control blood glucose levels in type 1 diabetic patients is multiple daily administrations of exogenous insulin accompanied with regular blood glucose checks. This treatment lacks precise glycemic control, and consequently for these patients results in an increased risk in long term complications such as, cardiovascular disease, neuropathy, retinopathy, and nephropathy [3]. Whole pancreas transplantation represents an alternative treatment. Advantageously, this provides a much better and natural blood glucose control, and diminishes the negative effects associated with long-term insulin injections. However, whole pancreas transplantation is limited by donor shortage, the availability of good quality organs, the life-long use of immunosuppressive medicines, and risk of tissue rejection, and it includes complicated abdominal surgery [4].

\section{Clinical islet transplantation}

Clinical islet transplantation (CIT) is a valuable alternative to whole organ transplantation and can be performed by a minimally invasive intervention. Lacy and Kostianovsky are considered the pioneers of clinical islet transplantation due to their discovery to correct hyperglycemia by isolating metabolically active, and intact islets from rodent pancreas [5]. This technique was improved in several human follow up studies, however only $9 \%$ of the islet recipients were insulin independent for less than one year. In 2000, the Edmonton group was able to achieve insulin independence in 7 patients by using a particular 
immunosuppressive therapy. The current Edmonton protocol is based on infusion of an adequate allogenic islet mass $(11,547 \pm 1604$ islet equivalents per kilogram of the recipient's body weight) into the portal vein of a diabetic patient. The material is usually collected from pancreatic tissue of more than one donor (Figure 1) in order to obtain a clinically relevant amount of good quality islets [6]. The initial clinical trials resulted in one year insulin independency of all 7 patients treated with the new protocol. However, less than 50\% of the recipients remained insulin independent for more than one year, and only $10 \%$ of the treated patients did not need exogenous insulin after 5 years [6]. Although this protocol provided a new way for treatment of this auto immune disease by $\beta$-cell replacement, improvements were clearly needed. A major problem is the loss of $60-70 \%$ of the islets within two weeks after transplantation. The loss of donor tissue is caused by a complex multifactorial adverse reaction to the transplant, involving an immediate blood mediated inflammatory reaction, allo and autoimmunity, lack of revascularization as well as high concentrations of glucose, lipids and immunosuppressive drugs in the liver. As a result, transplantation of donor islets has to be repeated several times before patients become fully insulin independent. Clearly novel methods are needed to enable the survival and function of transplanted islets over longer periods of time and make CIT a more widespread therapy.

Since 2000, many studies aim to improve the outcome of the initial Edmonton protocol by developing more effective immunosuppression therapies, and by improving the islet isolation procedures. The goal is to reduce the number of pancreas donors currently required to treat one patient to a single donor, however, still multiple transplantations are needed. Today, in some cases insulin independency was reached for more than 7 years, but still the majority of transplanted patients fall back to some form of exogenous insulin therapy. 




(C) 2005 Diabetes Research Institute, U. of Miami

Artist: Robert Margulies

Figure 1. Schematic islet transplantation into the portal vein. (Reproduced with permission from Diabetes Research Institute, University of Miami).

Understanding the factors which negatively influence CIT can help to significantly improve the efficiency of this therapy. The decrease of the injected islet mass is likely caused by mechanical stress during embolization of the islet in the liver's vasculature, the lack of oxygen and nutrients due to impaired vascularization, disruption of islet extracellular matrix (ECM) due to enzymatic degradation during isolation, the instant blood mediated inflammatory reaction (IBMIR), and longterm allo- and autoimmunity [7-10]. Although, many studies have been done, and are ongoing, to address the aforementioned obstacles there is still no definitive solution to tackle this multifactorial problem.

\section{Aim and outline of the thesis}

In this thesis, we report on several strategies to improve islet transplantation. One of our goals was to boost the endocrine function and survival of islets with a specific combination of ECM molecules relevant to islets. We also aimed to design an optimal supportive biocompatible scaffold for islets, which would support 
revascularization when implanted. Finally, we studied the in vivo performance of a scaffold at a potential extrahepatic islet transplantation location in rats.

\section{Chapter 2}

This chapter sets the stage by providing the reader with a comprehensive review to grant a contextual understanding of the pancreatic islet microenvironment. Specifically, we focused on key elements that can be used as a blueprint for the engineering of a suitable islet microenvironment in a future synthetic islet delivery device. This chapter focusses on the most important biological and bioengineering parameters that can influence the outcome of islet transplantation including; islet morphology, islet cell sources, intra islet cell interactions, the pancreatic islet extracellular matrix, biomaterials used for the preparation of scaffolds for islet transplantation, the immune reaction to transplanted islets, strategies to induce revascularization of a transplanted, and a potential in vivo site for transplanting an islet delivery device.

\section{Chapter 3}

Scaffolds are supportive structures that can be tuned to resemble the microenvironment of islets depending on the biomaterials applied. In this chapter the microstructure, physical and mechanical properties and hydrolytic degradation of two polymers, a poly(ester urethane) and polyethyleneglycol-terephtalate polybutylene-terephtalate (PEOT-PBT) were compared. These two distinct biomaterials were used to fabricate thin-film microwell array scaffolds for islet transplantation as described in the subsequent chapters.

\section{Chapter 4}

The biological interaction of biomaterials can be further augmented by incorporating specific biomolecules such as ECM proteins relevant to $\beta$-cells. In this chapter we investigated microcontact printing to prepare islets-sized areas coated with specific ECM proteins and study their influence on the function, survival and behavior of $\beta$-cell lines. We created a controllable pattern consisting of 100 
micrometer diameter small circles on different non-cell-adhesive substrates and coated these spots with different ECM molecules based on a method previously published by the Jonkheijm lab [11]. The size of the spots was chosen such that seeded $\beta$-cells will form so-called pseudoislets. We specifically investigated covalent coupling of fibronectin, collagen-IV and laminin-111 to the substrates, because these ECM molecules may support cell attachment and stimulate islet cells to form aggregates resembling native islets $[12,13]$.

\section{Chapter 5}

The islets native ECM contains an intricate combination of particular molecules, mostly derived from the basal lamina of vascular origin, and the peri-islet matrix. After having investigated the influence of single ECM molecules on $\beta$-cell lines (chapter 4), a medium throughput analysis was performed on different combinations of these ECM molecules on human islets. The goal was to preserve the natural morphology of islets and examine the effect of ECM molecules on glucose responsiveness. In addition, we applied these proteins as a coating on thinfim microwell array scaffolds, which can act as an islet delivery device, in order to improve the function of islets seeded into these devices. We used the two polymers decribed in chapter 3 as base biomaterials to produce the thin-film microwell array scaffolds. Both materials were functionalized with a similar set of molecules. Fibronectin, collagen type IV, laminin-111, or laminin-332 individually, and combinations of these, were investigated for their influence on human islets from multiple donors.

\section{Chapter 6}

In the previous chapters, we have described some of the necessary bioengineering steps to design and optimize a porous thin-film microwell scaffold platform for delivery of $\beta$-cells or islets. In this chapter, we describe an in vivo study on the biocompatibility of these microwell scaffolds at an intraperitoneal and subcutaneous site in Albino Oxford (AO) rats. In addition, we also performed an in 
vivo pilot study to investigate the performance and handling of the thin-film microwell array scaffold for islet transplantation in the intra peritoneal cavity. In this model, we transplanted Albino Oxford rat islets in diabetic immunocompromised nude Rowett rats.

\section{Chapter 7}

A major challenge encountered in islet implantation using microwell scaffolds is the nutrition and oxygen supply to the islets. Vascularization of the device after implantation is thus one of the decisive factors to improve viability and potentially enhance the glucose responsiveness of transplanted islets. The development of a dense vascular network directly after transplantation could lead to a high survival rate of endocrine cells. In an initial study we applied two strategies for controlled delivering of vascular endothelial growth factor (VEGF) from PEOT-PBT and poly(ester urethane) polymer films in order to promote neoangiogenesis. In the first method, layer-by layer (LBL) self-assembly of polyethylenimine (PEI), poly(sodium 4-styrenesulfonate) (PSS), heparin and VEGF was developed to apply a coating to the substrates. In the second method, the polymer films were functionalized by only VEGF and heparin. The release profile as well as the ability to induce tubular formation by these different constructs was studied in vitro using human umbilical vein cells (HUVECs).

\section{Chapter 8}

In the final chapter of this thesis the main conclusions of this work have been summarized and placed in perspective. Furthermore an outlook is provided for future work. 


\section{References}

1. Rathmann, W. and Giani, G. (2004) Global prevalence of diabetes: estimates for the year 2000 and projections for 2030. Diabetes care 27 (10), 2568-2569.

2. Ogurtsova, K. et al. (2017) IDF Diabetes Atlas: Global estimates for the prevalence of diabetes for 2015 and 2040. Diabetes Research and Clinical Practice 128, 40-50.

3. Nathan, D.M. (1993) Long-term complications of diabetes mellitus. New England Journal of Medicine 328 (23), 1676-1685.

4. Sutherland, D.E. et al. (2001) Pancreas transplantation for treatment of diabetes mellitus. World journal of surgery 25 (4), 487-496.

5. Lacy, P.E. and Kostianovsky, M. (1967) Method for the isolation of intact islets of Langerhans from the rat pancreas. Diabetes 16 (1), 35-39.

6. Shapiro, A.J. et al. (2000) Islet transplantation in seven patients with type 1 diabetes mellitus using a glucocorticoid-free immunosuppressive regimen. New England Journal of Medicine 343 (4), 230-238.

7. Carlsson, P.-O. et al. (2001) Markedly decreased oxygen tension in transplanted rat pancreatic islets irrespective of the implantation site. Diabetes 50 (3), 489-495.

8. Moberg, L. et al. (2002) Production of tissue factor by pancreatic islet cells as a trigger of detrimental thrombotic reactions in clinical islet transplantation. The Lancet 360 (9350), 2039-2045.

9. Eich, T. et al. (2007) Visualization of early engraftment in clinical islet transplantation by positron-emission tomography. New England Journal of Medicine 356 (26), 2754-2755.

10. d'Aleo, V. et al., Functional and survival analysis of isolated human islets, Transplantation proceedings, Elsevier, 2010, pp. 2250-2251.

11. Voskuhl, J. et al. (2014) Advances in contact printing technologies of carbohydrate, peptide and protein arrays. Current opinion in chemical biology 18, 1-7.

12. Kaido, T. et al. (2004) Regulation of human $\beta$-cell adhesion, motility, and insulin secretion by collagen IV and its receptor a1ß1. Journal of Biological Chemistry 279 (51), 53762-53769.

13. Bosco, D. et al. (2000) Importance of cell-matrix interactions in rat islet beta-cell secretion in vitro: role of alpha6beta1 integrin. Diabetes 49 (2), 233-243. 




\title{
Chapter 2
}

Tissue engineering of islets for the treatment of type 1 diabetes

\author{
E. Hadavi \\ J. Leijten \\ H.B.J. Karperien \\ A.A. van Apeldoorn
}




\section{Abstract}

Transplanting islets into a patient's portal vein is currently the most promising method to treat type one diabetes. However, the long-term efficiency of this method has only been demonstrated in a small number of the treated patients. In the majority of patients, the treatment ultimately fails and they will still suffer from labile blood glucose control. Although it is known that multiple factors play an important role in the loss of donor cells, such as the immediate blood mediated inflammatory response, allo and auto immune reaction, poor revascularization and high levels of glucose, lipids and immunosuppressive drugs. Another factor, which more recently is being studied, is the effect of islet isolation, which can induced damage to the $\beta$-cell's microenvironment. Specifically, in order to isolate islets the pancreas is perfused with collagenase, this leads to enzymatic digestion of the islets extra cellular matrix causing loss of the general extracellular matrix (ECM) network architecture, and supportive ECM molecules. Another important factor, is insufficient rapid vascularization after transplantation leading to poor mass transport of oxygen and nutrients to the metabolically very active $\beta$-cells leading to cell stress, apoptosis and loss of function. The ECM in islets consists predominantly of a basement membrane associated with the dense microvasculature surrounding the endocrine cells. Consequently, it is expected that islet transplantation can be significantly improved by re-establishing the islet microenvironment. Here, we review the state-of-the-art in engineering islet microenvironments, and provide a comprehensive understanding of the relationship between pancreatic islet cells and its surrounding microenvironment within the islet. Additionally, we highlight the progress and challenges related to the biological, biochemical, and biophysical aspects of engineering the islet micro-environment for transplantation purposes. 


\section{Introduction}

Type 1 diabetes mellitus (T1DM) is a disorder that results in autoimmunemediated destruction of pancreatic islet $\beta$-cells [1]. The consequent damage or loss of the majority of $\beta$-cells leads to insulin deficiency and a pathologically high level of blood glucose. Based on the world health organization's global report of 2016, more than 422 million people are suffering from diabetes and it is estimated that $5-10 \%$ of all diabetes cases are T1DM [2].

Current therapies focus on diet, exercise, and medication [3]. Insulin injections either by insulin pumps or subcutaneous injection are mostly used to effectively lower blood glucose levels. However, this therapy does not avoid the incidence of diabetic related microvascular disorders such as retinopathy, nephropathy, and neuropathy, which are the main causes of morbidity and mortality of diabetic patients [4]. Whole pancreas transplantation is an alternative treatment, but faces several obstacles including donor shortage, life-long use of immunosuppressive medicines, and risk of tissue rejection [5]. Allogeneic islet transplantation is another alternative therapeutic method to treat patients with type 1 diabetes.

Although allogeneic islet transplantation was initially regarded as a promising therapy, its application has been limited due to several obstacles, including shortage of islet donors, early loss of islets at the intrahepatic transplantation site, and risk of portal vein thrombosis or bleeding. Specifically, $\sim 60 \%$ of the transplanted islet mass is lost within 3 days post-implantation and only $\sim 10 \%$ of patients stay insulin independent for up to five years post-transplantation [6]. One of the factors that is postulated to negatively impact on islet survival is the enzymatic digestion required to isolate the donor islets. It is widely accepted that the enzymatic digestion of the islet's extracellular matrix (ECM) during the isolation procedure causes degradation of the ECM and a loss of relevant ECM proteins, the disruption of the islets dense microvascular network, which negatively impact on the endocrine function and cell survival [7]. 
Re-engineering the islets' microenvironment using a bioengineering strategy could aid in re-establishing these important biological factors by recreation of the islets' intercellular niche. Engineering a well-defined microenvironment may not only lead to improved cell survival and endocrine function, but could perhaps also positively affect beta cell differentiation in case of induced pluripotent or embryonic stem cells. A profound understanding of the islet ECM architecture, its biomechanical properties and biophysical characteristics, and biological processes such as cell signaling and homeostasis is crucial in the design of such a microenvironment.

In recent years, several groups have reviewed the parameters involved in the islet microenvironment to improve the outcome of islet transplantation [8-13]. These papers focused on a potential cell source for $\beta$-cell replacement, islet-ECM interactions, development of scaffolds for islet transplantation and controlled revascularization of islets [8-13]. However, a comprehensive overview on the influence of microenvironmental parameters on the function and efficiency of the pancreatic endocrine cells has remained lacking. In this review, we focus on key elements that define an islet's microenvironment in order to provide a blue print for the engineering of proper islet microenvironments that could lead to better islet transplantation outcomes.

\section{The islet's cellular composition}

Only $2-3 \%$ of the total pancreas mass is constituted of islets of Langerhans. Islets are composed of 5 endocrine cell types that are defined by the hormones they release and the expression of genetic markers. Specifically, islets contain glucagonproducing a-cells (15-20\%), insulin producing $\beta$-cells (60-80\%), somatostatin producing $\delta$-cells (5-10\%), ghrelin expressing $\varepsilon$-cells, and pancreatic-polypeptide (PP) producing cells $(<2 \%)$. The continuous interaction between these cells is crucial for maintaining systemic glucose hemostasis [14, 15]. Interestingly, the islet size is similar in larger animals, like humans, pigs and primates, compared to 
smaller animals like rabbits and mice. However, the larger species have a larger number of islets to compensate for a larger body volume. This suggests that the functionality of islets is related to a certain optimal size and cell number in relation to organism's metabolism [16]. Additionally, the cytoarchitecture of human, porcine and primate islets differ from those in rodents [17]. Rats and mice islets typically have core-mantle structure with $\beta$-cells located in the core. The $a, \delta$, and PP cells are located on the outside, while the number of a-cells can slightly vary depending on the location of islets in the pancreas $[17,18]$. In contrast, $\beta$-cells in human islets seem to be randomly dispersed with the other endocrine cell types [19]. In addition, the number of both $a$ - and $\beta$-cells can differ based on their location in PP-rich (ventral) or PP-poor (dorsal) regions of the human pancreas [20, 21].

\section{Engineering new $\beta$-cells}

Replenishing $\beta$-cells either by in vivo regeneration, or in vitro expansion of cells and then transplanting them into diabetic patients could potentially solve the problem of pancreas donor shortages. Understanding the mechanisms that regulate the neogenesis of $\beta$-cells can result in better glucose positive $\beta$-cell replenishment therapies [22]. New $\beta$-cells can be obtained in vitro via several methods including trans differentiation of primary cells and reprogramming of stem cells [22]. For instance, in vitro transformation of liver cells into $\beta$-like cells has been achieved by over expression of a master gene controlling pancreatic development (PDX1) in hepatic cells. However, this technique can induce formation of both pancreatic endocrine and exocrine cell types [23].

There are different hypotheses regarding the origin of islet cells (Figure 1A). The neogenesis of $\beta$-cells could originate from ductal or acinar cells [24], or intra-islet cells including a- or $\beta$-cells. Since duct cells surround islets in a mature pancreas, it is assumed that progenitor cells are residing amongst ductal cells, which can differentiate into endodermal islet cells and ultimately into $\beta$-cells $[25,26]$. Hui et 
al. reported on the differentiation of a human pancreatic ductal cell line (PANC-1) into insulin secreting $\beta$-cells, in the presence of Glucagon like peptide 1 (GLP-1). However, no expression of the PDX1 gene was found [24]; PDX1 expression seems essential for differentiation towards insulin producing $\beta$-cells [27].

Another beta cell source which was studied are acinar cells. In these studies, acinar cells were either stimulated by epidermal growth factor (EGF) and ciliary neurotrophic factor (CNF) [28], or by over expression of three endocrine transcription factors (NGN3, PDX1 and MAFA) [29]. Although, the generated $\beta$ cells improved the blood glucose levels in mice, and expressed $\beta$-cell-specific genes, while typical islet morphological characteristics were observed, they did not form natural islet-like clusters [29]. Another strategy involves the conversion of $\beta$-cells from a-cells using genetic manipulation achieved by ectopically expressing Pax4 or Nkx6.1, two $\beta$-cell specific reprogramming factors. Using this approach functional $\beta$ cells at least at an early age were generated that were able to repopulate the islets of diabetic mice and normalize blood glucose levels [30-33].

In addition to islet cells and pancreatic cells to create new insulin producing cells, rare pancreatic multipotent precursor (PMP) cells can also be used to generate mature and glucose responsive $\beta$-cells. PMP cells that express insulin, and low levels of the glucose transporter (Glut2), have been identified in mice and human islets $[34,35]$. More recently promising results have been obtained in the differentiation of induced Pluripotent Stem cells (iPS) or embryonic stem cells into functional insulin producing $\beta$-cells that can restore normoglycemia upon transplantation in mice [36-41] (Figure 1A).

Differentiation of islet cells from progenitor cells could contribute in the replacement of defected $\beta$-cells and thus functional islets. This can extend clinical islet transplantation to millions of patients and bypass the problem of donor shortage. 


\section{Islet cell interactions}

\section{- $\quad a-$ and $\beta$-cells}

The islet cells communicate through homotypic or heterotypic cell-cell interaction. The close cellular proximity is essential for a normal pattern of insulin secretion as well as islet survival in the long term (Figure 1B). To effectively engineer functional islets, it is essential to understand the relevant cellular interactions taking place within islets. The importance of the adhesion molecules, and the density and size of the cell aggregates of the islet have been investigated in a few studies [42, 43].

In an islet, $\beta$ - and a-cells have a close relationship. The a-cells are responsible for the regulation of insulin and glucagon at low blood glucose levels. Glucagon gives a positive autocrine feedback to its own secretion. Glucagon activates a-cells ionotropic glutamate receptors (iGluR), which leads to influx of $\mathrm{Na}^{+}$and $\mathrm{Ca}^{2+}$ and results in depolarization of the a-cell's membrane and opening of the voltage gated$\mathrm{Ca}^{2+}$ channel. Consequently, more glucagon, and glutamate, are released by activation of $\mathrm{Ca}^{2+}$-driven exocytosis $[44,45]$. $\beta$-cell can secrete factors such as insulin, GABA and $\mathrm{Zn}^{2+}$, which all can have an inhibitory effect on glucagon secretion (Figure 1C). Insulin can modulate the a-cell's secretory function by decreasing the sensitivity of K-ATP channels to ATP. Moreover, GABA secreted by $\beta$-cells activates the $\mathrm{Cl}^{-}$inward membrane current in a-cells, and results in the hyperpolarization of the a-cell's membrane, causing the voltage dependent $\mathrm{Ca}^{2+}$ channels to close, this subsequently inhibits the secretion of glucagon [46-49]. $\mathrm{Zn}^{2+}$ ions can reduce the electrical activity of a-cells and therefore decrease glucagon secretion [50]. Moreover, it is reported that a-cells in specific situations, for instance when islets are under metabolic stress [51] or exposed to inflammatory stimuli [52], will produce GLP-1. GLP-1 can increase insulin secretion, insulin gene expression, inhibit $\beta$-cells apoptosis, and lead to expansion of islet cell mass. GLP1 can thereby improve the endocrine function and preserve the morphology of islets 
$[53,54]$. This data indicates the importance of a-cells in the engineering of functional islets. In several attempts to recreate islets from single cells, various ratios of $\alpha$ - and $\beta$-cells, including $1: 2,1: 1,2: 1,1: 3,1: 8$, and only $\beta$-cells were used. The highest insulin secretion was found at a 1:8 ratio of a and $\beta$-cells [55]. Although $\beta$-cells are the primary actor in insulin secretion for a successfully engineered islet one should perhaps consider a-cells as well due to their cross talk with $\beta$-cells.

\section{- $\delta$-cells}

The $\delta$-cells produce somatostatin which is transported via the local microcirculation and interstitium, and has an inhibiting effect on both insulin and glucagon secretion of neighboring $\beta$ - and a-cells $[56,57]$. Somatostatin interacts with $\mathrm{G}$ protein-coupled receptors (GPCRs), activating $\mathrm{K}^{+}$-channels and inhibiting the $\mathrm{Ca}^{2+}$-independent pathway causing hyperpolarization of the $\beta$ - and a-cell membrane. Moreover, somatostatin can inhibit adenylate cyclase (AC), which decreases production of cAMP affecting protein kinase A activity leading to a decrease in glucagon and insulin secretion $[58,59]$. Ramachandran et al. added $\delta$ cells to generate islets for therapeutic purposes [60]. They reported that if $a-, \beta-$ and $\delta$-cells with a similar cellular composition as native islets were re-aggregated in micromolds the engineered islet aggregates remained responsive to varying glucose levels. However, follow-up in vivo experiments demonstrated fluctuation of blood glucose levels and stable normoglycemia was not achieved. Therefore, more research is necessary to understand the importance of $\delta$-cells for islet engineering.

\section{- $\varepsilon$-cells}

$\varepsilon$-cells locally regulate insulin release by producing the hormone ghrelin. The number of $\varepsilon$-cells reduce after birth, and only small numbers of $\varepsilon$-cells remain in adult islets [61]. The cells inhibit insulin release by interaction with $\mathrm{G}$ protein coupled receptors (GHS-R) on $\beta$-cells $[62,63]$. Since the population of $\varepsilon$-cells in native mature islets is relatively low and these cells have an inhibitory effect on insulin secretion, research into engineered islets for treatment of type 1 diabetes 
hardly includes $\varepsilon$-cells. However, injection of an GHS-R antagonist in rats indicated a rise in insulin concentration in their blood [64].

The presence of sufficient numbers of $\beta$-cells in islets is necessary for the proper function of islets. A study from Wojtusciszyn et al. indicated that dispersed, or a low density of, $\beta$-cells can decrease the amount of insulin release. When the number of $\beta$-cells is low ( $<50 \beta$-cells), less cell-to-cell contact is made and their sensitivity to release insulin at high glucose levels is decreased [65-67]. On the other hand, a high number of $\beta$-cells (100-200 $\beta$-cells) does not necessary lead to an increase in insulin secretion, suggesting that aggregates containing a high number of $\beta$-cells are not necessarily better [65]. Large aggregates $(>150 \mu \mathrm{m})$ are more susceptible to necrosis due to the lack of nutrients and oxygen in the core of these aggregates. Having aggregates composed of too many $\beta$-cells can result in a reduction of insulin secretion [66, 68-70]. Ideally, an optimal $\beta$-cell number of 50-100, and aggregate sizes with dimensions between 50-125 $\mu \mathrm{m}$ are preferred if one wants to create islets with a proper sustainable endocrine function $[65,71]$. Additionally, the study of Wojtusciszyn et al. demonstrated the importance of heterogeneous activity of the $\beta$-cells in terms of insulin release [67]. Using $\beta$-cells aggregates they found that around $20 \%$ of all $\beta$-cells are responsible for more than $70 \%$ of the total amount of secreted insulin. In addition, it is suggested that the pulsatile secretory behavior of $\beta$-cells aggregates is influenced by multiple factors such as, the communication between $\beta$-cells, the concentration of glucose, the mode of glucose delivery to the cells, and cAMP-dependent mechanisms [72].

\section{Cell adhesion and $\beta$-cells function}

$\beta$-cell function is also affected by neural cell adhesion molecules (NCAM), cadherins, gap junction protein connexin-36, Eph receptor, ephrin ligands, and secreted insulin [73-76]. E-cadherin is a calcium dependent transmembrane protein that facilitates cell-to-cell adhesion. It has a role in islet formation, insulin release, and gap junction communication. The cadherins on the extracellular domain of 
adjacent cells tend to form a calcium-dependent homodimer. It was shown that this process facilitates cell adhesion and formation of aggregates during the development of the mouse pancreas. Moreover, it controls gap junctional communication [77-79]. NCAM is another cell adhesive molecule that influences homophilic and heterophilic cell to cell interactions. Lack of NCAM results a-cells separating from islets, and abnormal islet morphology causing a decrease in insulin secretion [80, 81]. Gap junctions produce tubular intracellular channels between $\beta$-cells in order to exchange cytoplasmic ions and metabolites between the adjacent cells [82]. These gap junctions are important for storage and release of insulin, and fine-tuning biosynthesis [83]. Eph and ephrin are expressed in human and mouse islets. They both communicate with each other and initiate bidirectional signaling. At low glucose levels, EpH suppresses insulin release while at high glucose levels ephrin sends signals to increase insulin release [75]. The roles of adhesion molecules, gap junctions, protein receptors and their ligands, as well as interactions between individual endocrine cells must be intensively analyzed to facilitate effective reconstitution of engineered islets for use in regenerative medicine.

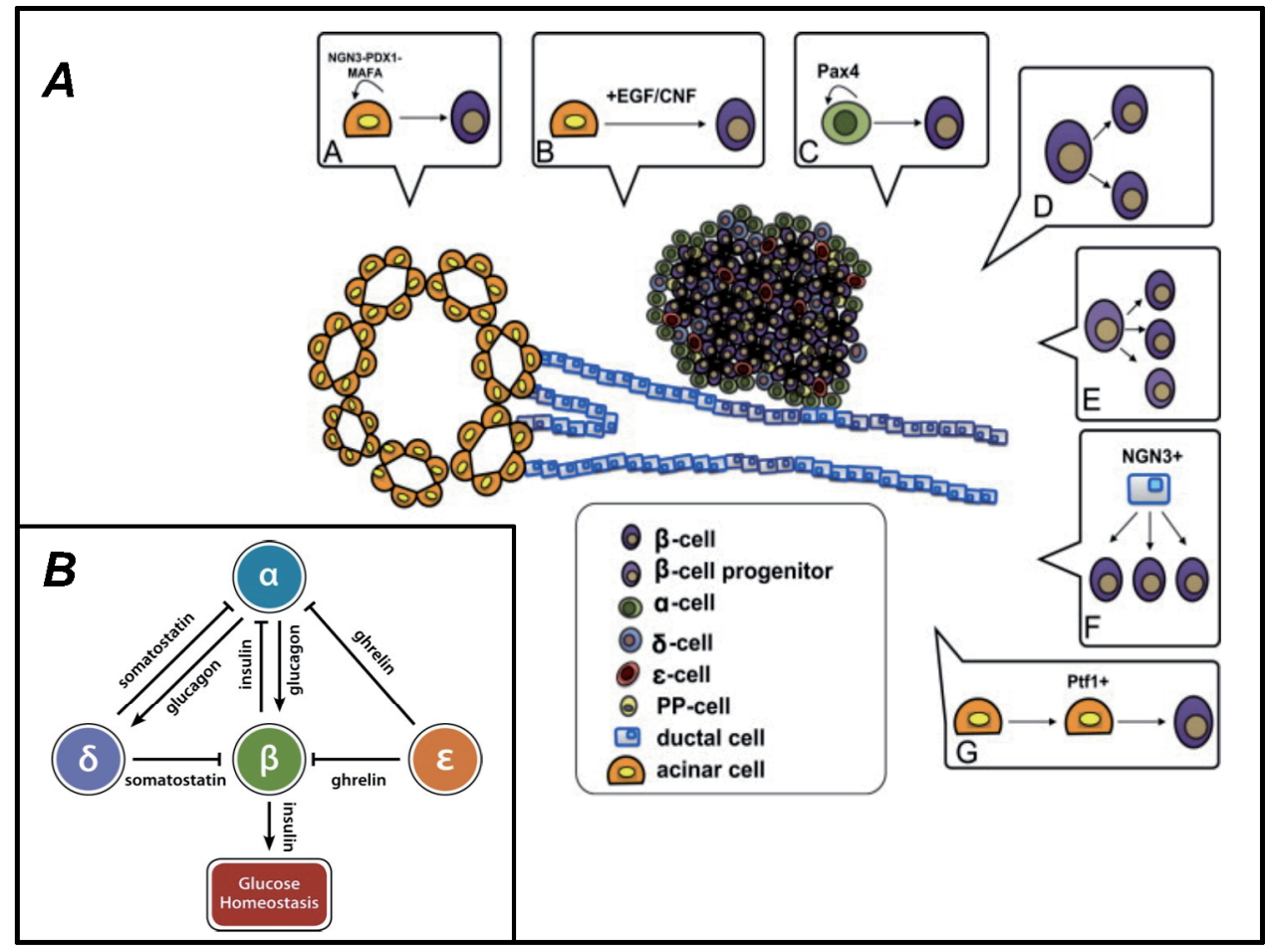




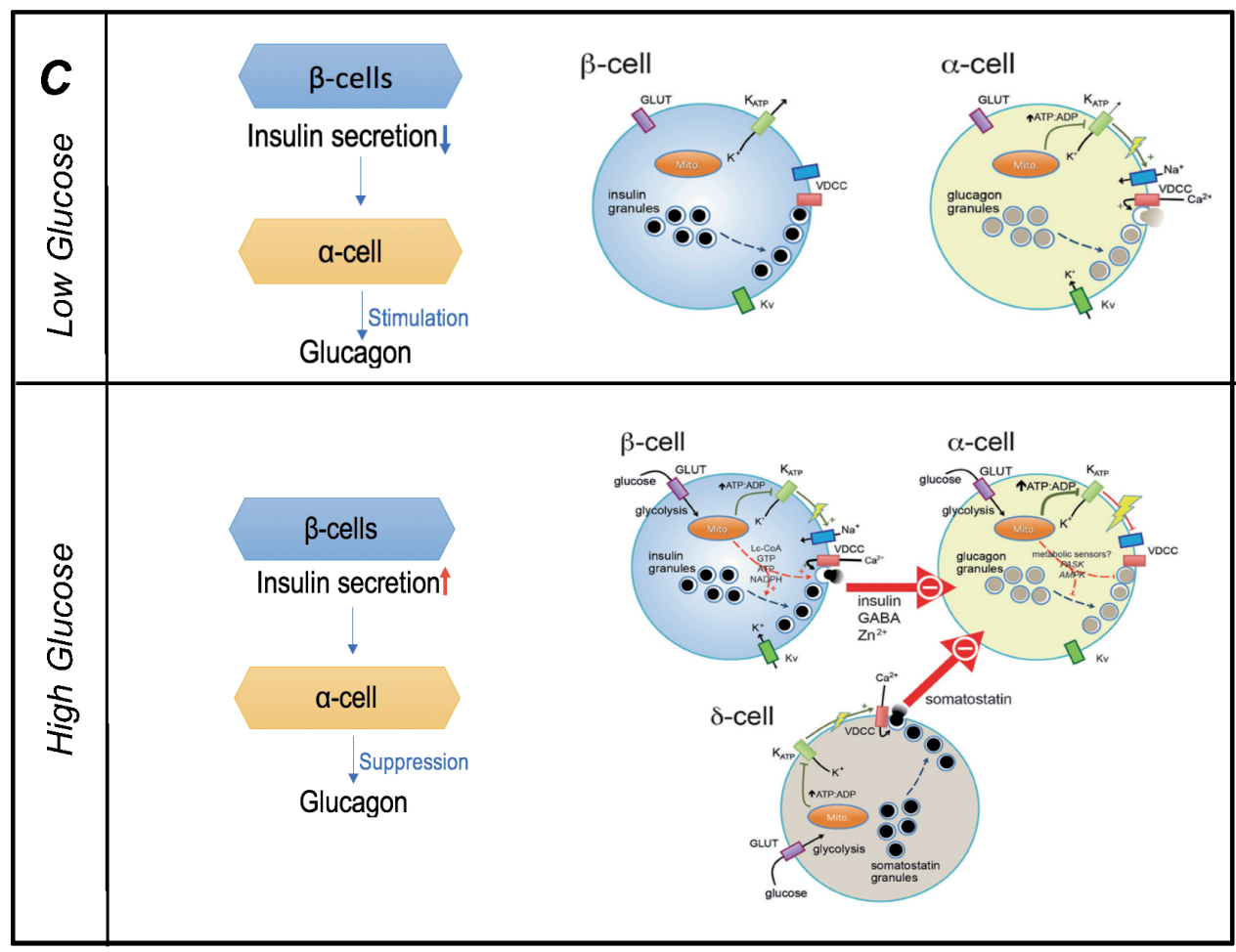

Figure 1. Islet cells interactions. A) Potential ways of $\beta$-cell regeneration, B) interaction between pancreatic islet cells, C) $\beta$-cell- $\beta$-cell interaction controls insulin release at low and high glucose level. Adapted from[46, 84, 85]

\section{Extracellular matrix molecules in pancreatic islets}

The extracellular matrix (ECM) is a highly important constituent in bioengineering due to its structural and biochemical supportive roles [86] and its influence on many cellular processes including proliferation, differentiation, adhesion, and cell-cell communication [87]. ECM is composed of distinct proteins, polysaccharides, and lipids and each tissue has its own specific composition [87]. ECM molecules can mediate their effect either directly on cells or by cross talk between signaling pathways and cells. 
Before engineering any kind of tissue, having a comprehensive knowledge regarding the types of the existing and dominant molecules in ECM and their function and influence on cells, is essential. Here, we provide a detailed survey of the ECM of pancreatic islets that should be considered to better predict the outcome of engineered islet delivery devices.

In islets, two major compartments constitute the ECM: the stromal ECM and the basement membrane (BM). The stromal ECM provides mechanical stability and is organized as a thin layer located immediately adjacent on the outside to the periislet BM as well as surrounding large ducts and blood vessels [88]. Stromal ECM of islets is characterized by the presence of fibrillar collagens such as Collagen type 1 (Col1) and type 2 (Col2), non fibrillar collagen type 4 (Col4) and glycoproteins like fibronectin (FN), tenascin, vitronectin, proteoglycans like chondroitin or dermatan sulfate, and fibrillin-2 and matrilin-2 [89].

The BM acts as a barrier between epithelial and endothelial layers inside islets by surrounding the islet and peri-islets microvessels. It consists of laminins (LN) and Col4 linked by nidogen/entactin molecules [90]. Studies on mouse islets have shown that the endocrine cells interact directly with the vascular endothelial BM [91]. In contrast, human islets are surrounded by two distinct layers of BM. These layers are endocrine $\mathrm{BM}$ and perivascular BM. The islet BM layer surrounds the perivascular BM, including Col4, LN, nidogen, and perlecan [11]. Several studies have focused on the role and influence of ECM molecules like FN, Col, LN, and vitronectin to augment the efficiency of engineered islet or $\beta$-cells for islet transplantation.

\section{- Fibronectin}

Fibronectin (FN) is a dimeric glycoprotein with high molecular weight that exists in soluble (in plasma) and fibrillary forms [92]. FN has different domains that can non-covalently interact with molecules such as heparin, collagen, chondroitin sulfate, and integrins, as well as FN itself [93]. FN is localized in the adult islet periphery, islet ductal pole and perivascular area and its presence is usually 
accompanied with Col1, Col3, Col4, and LN. Islet cells usually have interaction with ECM molecules via heterodimeric transmembrane receptors consisting $a$ and $\beta$ subunits, the integrins [11]. The most well-known peptide signaling sequence of FN is arginine-glycine-aspartic acid (RGD), which is recognized by different integrins such as $a 3 \beta 1, a 4 \beta 1, a 5 \beta 1, a 4 \beta 7, a 8 \beta 1, a v \beta 1, a v \beta 3, a v \beta 5, a v \beta 6$, and aIIbß3 [94].

Cirulli et al. indicated a significant decrease in the number of insulin positive cells by transplanting fetal islets in nude mice, which were treated with a competitive RGD blocking peptide [95]. Moreover, it is reported that interaction of integrins with the ECM influences undifferentiated human fetal pancreatic epithelial cell migration in the early stages of islet development $[95,96]$ and protects, in mature human islets, cells from apoptosis [97]. The addition of soluble FN to islets in suspension cultures can increase islet survival about 1.5-, and insulin secretion about 2-fold, compared to untreated suspension cultures [98]. In contrast, cells from dissociated porcine islets showed strong adhesion to, and spreading of adhered cells, on FN coated substrates with no significant influence on their endocrine function $[96,99]$. A study on the effect of FN on human cells revealed strong adhesion of $\beta$-cells to Fib, with low rates of survival and migration of both adult and fetal cells [96]. In addition to integrin-binding RGD, there are some nonintegrin receptors of FN consisting of dystroglycan and syndecan [100]. The function of dystroglycan and syndecan on islet cell is not known well. It is reported that syndecan may have a role in binding of islet cells to Col and LN [100]. Dystroglycan has been implicated to play a role in differentiation and survival of laminin-1-induced $\beta$-cells in fetal mouse pancreas [101] but there is not any information in order to its role in binding with FN.

\section{- Collagen}

Collagens represent about $30 \%$ of the total amount of the body's proteins. Collagens not only provide mechanical support, but also have a role in cell adhesion, differentiation, morphogenesis, and wound healing [102, 103]. The 
different types of collagen identified in islets are Col1, Col2, and Col5, which have a fibrillar structure, Col4, which forms non-fibrillar networks, and Col4, which forms beaded filaments $[89,104]$. Based on quantitative analysis of collagen subtypes in peripheral ECM islets, Hughes et al. reported that the prevalence of Col4 is significantly higher than Col1 and Col4 in the adult pancreas [105]. Also, among all sub-types of collagen, Col4 along with FN and vitronectin were identified in early precursor islet tissue emerging from ducts. The combination of Col4 and LN was also identified in immature developing islets [95], as well as the perivascular $\mathrm{BM}$ of mature islets in direct or close contact with $\beta$-cells [106]. However, Kaido et al. reported that most of the binding interaction of $\beta$-cells around microvessels is with LN rather than Col4 [107]. Collagens interact via integrins, which include $a 1 \beta 1, a 2 \beta 1$, a3 $\beta 1$ which can bind and induce certain signal transduction pathways [108]. Blocking the $\beta 1$ integrin receptor in islets cultured on Col4 coated well plates results in inhibition of cell adhesion to the substrate, leading to a decrease in insulin expression, and increase in apoptosis [109]. Islets cultured on well plates coated with Col4 show a significant increase in glucose responsiveness and insulin secretion. However, the physiological effect of Col4 on islets is not entirely clear [97]. Also, comparing the influence of ECM coated scaffolds, with Col4, FN, or LN, on encapsulated islets after transplantation in mice demonstrated enhanced function of islets with Col4 [110]. Col1 has predominantly a role in mediation cell adhesion, binding to other ECM molecules, and interaction of cells with tissue calcification factors and glycation [111].

Col4 can also bind with other ECM molecules such as LN via intermediary glycoproteins nidogen, or entactin, or via direct interaction to heparin sulfate proteoglycans [112]. LRE, a LN derived recognition sequence peptide, in combination with Col4 seems to enhance insulin secretion synergistically [113]. Nagata et al., reported that rat islets cultured in Col1hydrogels in combination with Col4 improves insulin secretion [114]. They also stated that culture of rat islets in Col1 hydrogels mixed with Col2, Col4, and LN, improves their survival [115]. Nevertheless, this combination had no effect on the mRNA expression of insulin, 
glucagon and somatostatin[115]. They also did not observe any effect of collagen on the secretion of the hepatocyte growth factors (HGF), interleukin (IL)-1a, and $\mathrm{IL}-1 \beta$ by the islets [115].

The pancreatic cells are also known to bind arginine-glycin-aspartic acid (RGD) sequences on ECM molecules by integrin receptors $a_{3} \beta_{1}, a_{5} \beta_{1}$, and $a_{v} \beta_{3}$, which can promote survival of isolated islets. Although, it is demonstrated that the RGD domain in collagens are inactive in the native conformation, enzymatic digestion makes the RGD sequence accessible for isolated islets [11]. Addition of ECM molecules to scaffolds, especially Col4, can improve the function of transplanted human islets in mice $[116,117]$. Taken together, Col1 and Col4 seem to possess a higher potential to improve the function and survival of transplanted islets as compared than any of the other collagen types.

\section{- Laminins}

Laminin (LN) form a major $(\sim 80 \%)$ component of the BM were it provides structural support and helps regulate cell processes like proliferation, differentiation and cell migration [109, [118]. LN is a heterotrimeric glycoprotein that consists of 3 different polypeptide chains termed $a, \beta$ and $\gamma$. For instance, LN111 consists of $a 1, \beta 1$ and $\gamma 1$ polypeptide chains. Up to now, 18 different isoforms of LN have been identified in humans and animals. Various isoforms of LNs are differently expressed in different basement membranes. The laminin, a2, a4, $\beta 1, \beta 2, \gamma 1, \gamma 2$, and $\gamma 3$ chains are commonly found in the peri-islet BM of both human and mouse islets, but a5 chains are only expressed in humans, while a4 chains are only found in mice $[90,119,120]$. The Laminins found in BM surrounding blood vessels consist of either a 4 and a5 chains, together with $\gamma 1$ and $\beta 1$ chains in both humans and mice $[90,121]$. These polypeptide chains can play an important role in $\beta$-cell adhesion, proliferation, and insulin secretion [122]. Jian et al., reported that LN111 promotes survival of islets and differentiation of fetal pancreatic cells to $\beta$-cells via a-dystroglycan integrin [123]. LN111 is also involved in the proliferation of pancreatic precursor cells via $a_{6}$ integrins [101]. 
Furthermore, the role of LN511 in preservation of the human $\beta$-cell phenotype was reported from in vitro studies [124]. LNs can bind to other ECM molecules and form crosslinks.

It was long assumed that LN332 was not present in islets, however Parnaud et al. indicated that LN332 is associated with a-cells of rats and human islets [125]. The authors were able to show that blocking the interaction of LN332 with its associate integrin $(\beta 1)$, reduces glucose-stimulated insulin secretion [125]. In addition, the presence of LN411, 421, 511, and 521 in the BM of human islets was reported [91]. The role of these laminins is not known well. Nikolova et al. reported that LN411 has a role in upregulation of Insulin gene expression of $\beta$-cells [122]. Brandhorst et al. reported that LN411 and 511 increase the viability of islets [126].

LNs in addition to their well known interactions with integrins also can have interaction with non-integrin receptors such as dystroglycan and LN-receptor-1 [101, 127]. The interaction with non-integrins is important for survival, differentiation and morphogenesis of islets [101]. To date, some peptide sequences are identified on $\beta 1$ chain of LN111 and LN511 including YIGSR [128], PDSGR [129], RYVVLPR [129], and LGTIPG [128] which play a role in these interactions. Some other cell binding sequences were identified in the a1 chain of LN111, including IKVAV [130], IKLLI [131], and VAYI [132]. However, the effect of these sequences on islet cell function and behavior has remained largely unknown. Regardless, Liacua et al., reported that the laminin derived sequences of RGD and PDSGR peptides could improve the insulin release and survival of transplanted islets when applied at concentrations ranging from 0.01 to $1.0 \mathrm{mM}$. Moreover, they observed a synergistic effect of the LRE sequence of LN combined with Col4 on insulin release [113]. Interestingly, it was reported that the LN a5 chain has affinity to Lutheran (Lu) glycoprotein of islet cells in human [133, 134], while this affinity was not observed in rodent islets. This observation indicates that the effect of LNs may differ in various species. Overall, studying the influence of LN on islet cells can give insights regarding its application for islet engineering purpose. Among all 
laminins, laminin $111,332,411-421$, and $511-521$ are the most promising to include in the ECM of an engineered islet.

\section{- Vitronectin}

Vitronectin is a glycoprotein that consists of 3 different domains. One domain with an RGD sequence is involved in the binding of integrin receptors av $\beta 1$, av $\beta 3$, avß5, and aIIb $\beta 3$ [135]. The second domain binds to plasminogen activator inhibitor-1 (PAI-1), which could regulate proteolysis. The third domain binds heparin following its activation by the thrombin-antithrombin III complex [136]. Within islets, vitronectin only is present during fetal development [95]. It was reported that vitronectin is present during development of islets, but it is absent in mature islets [137]. Since integrin av $\beta 1$ is essential for the migration of fetal $\beta$ cells, it is suggested that vitronectin has a role in islet morphogenesis [100]. Despite the natural occurrence of vitronectin in islets, it has unfavorable effects on engineered islets. Culturing $\beta$-cells derived from both fetal and adult islets on human vitronectin diminished the gene expression of insulin, while a reduction in insulin content was observed in fetal cells [107].

\section{Decellularization - recellularization technology}

Decellularization leaves the native composition and structure of the ECM of tissue largely intact and provides a minimal immunogenic scaffold. Decellularized tissues are biocompatible and still contains basic proteins, ECM molecules and matrix bound growth factors that were originally present although concentrations may be lower than in native tissue. It has the potential to provide an ideal biomaterial platform for regenerative medicine [138-140]. Carlo et al., indicated that on an acellular pancreatic matrix rat islets were functional and viable for 42 days of culture. In contrast, rat islets cultured on liver derived ECM showed a progressive reduction in endocrine function [141]. Interestingly, when the decellularized pancreatic matrix was incorporated with islets in a poly (vinyl alcohol) (PVA)/ poly (ethylene glycol) (PEG) tube hyperglycemia was reduced [141]. 
Conrad et al., reported preserved cell viability and function of human islets cultured in a recellularized murine pancreatic matrix, in vitro [142]. Interestingly, Mirmalek-Sani et al. demonstrated a successful regeneration of pancreatic tissue using a decellularized porcine pancreas by seeding human stem cells and porcine pancreatic islets [143]. Their approach using human stem cells with porcine pancreatic ECM may represent a platform for regeneration of insulin producing cells [143].

More investigation in the role of combination of ECM molecules and their combinatorial effects are needed to engineer more realistic islet microenvironments.

\section{Biomaterial technology for islet engineering}

ECM molecules by themselves are unlikely to provide all factors such as mechanical characteristics, stiffness, elasticity and strength which contribute significantly to the differentiation, proliferation, survival, migration, and behavior of cells [144]. These characteristics can be engineered via the use of biomaterials. Nowadays, there is a wide range of natural and synthetic materials or combinations available that can be used for building a scaffold structure that could be combined with ECM molecules. Naturally derived materials are generally purified ECM molecules or mixtures of them (matrigel) as well as decellularized organs. Natural materials can also be derived from plants or animal constituents such as alginate, chitosan, silk, and agarose. The advantages of natural components are largely based on their biocompatibility and biological activity. In contrast, the advantages of synthetic materials are based on their controllable physicochemical properties as well as manufacturing variability. It is important to select an appropriate polymer with desired physical, mechanical, and topological properties as a scaffold material for transplantation. Scaffold materials may affect the signaling pathways, function, and viability of the $\beta$-cells as well as the rate of vascularization and immunosuppressive effect. 
Smink et al., investigated the influence of different types of polymers on islet viability and functionality. The results revealed that the type of polymer used can affect the function of human islets. The experiments were performed by seeding human islets on glass petri dishes spin-coated with different synthetic polymers, such as poly(D,L-lactide-co-e-caprolactone), 4000PEOT30PBT70 block copolymer, and polysulfone [145].

Lacy et al., studied the influence of surface roughness on immune reactions to rat islets in 2 different types of poly(acrylontrile-co-vinyl chloride) hollow fibers, after encapsulating the islets first in alginate. One of the fibers had a smooth outer wall and the other was fenestrated. The fibers were implanted in intraperitoneal and subcutaneous sites of non-immunosuppressed mice. A similar biocompatibility for both fibers was reported. Moreover, the immobilization process did not affect the transport of nutrients and insulin in vitro. Improved immune-protection and control of glycemic levels were observed for the smooth fibers than for the fenestrated fibers [146]. Other studies indicated that the smooth and continuous outer wall of scaffolds could minimize the host fibrotic response [146-148].

\section{- Macroencapsulation}

Macroencapsulation is based on enhancing the function and survival of transplanted islets. The advantage of macroencapsulation is that a large number of islets can be incorporated in the scaffold with sufficient mechanical integrity. Furthermore, the scaffold can easily retrieved in case of severe adverse responses after implantation. However, the large size can cause some problems like folding and fragmentation after implantation [149]. Islet aggregation and fusion after immobilization in the capsule are other obstacles for some of these devices. Moreover, in most of the designs the use of immunosuppressive medication after transplantation is still needed. Macroencapsulation devices are usually designed as micro-porous, macro-porous, tubules, planar sheets or microwell implants (Figure 2A, B and C). 
Tubular membranes are usually based on hollow fibers with immobilized islets inside of the fibers. These constructs are usually applied as extra-vascular as well as intra-vascular devices. Lanza et al., compared the efficiency of extra-vascular and intra-vascular hollow fiber systems, which were fabricated from permselective acrylic membranes. The results indicated shorter survival times of the transplanted porcine islets in diabetic rat for the extra-vascular than intra-vascular system. This was mainly due to the shortage of oxygen and nutrients as well as diffusion of metabolites [147, 150]. Moreover, they demonstrated long-term survival of allogeneic dog islets transplanted in diabetic pancreatectomized dogs using the permselective acrylic membranes fibers. A device transplanted into the peritoneal cavity of dogs for 51 to 81 days indicated graft survival, glucose control and adequate insulin release of the islets [151]. Prevost et al. stated that transplanted islets in rats using AN69® (polyacrylonitrile-sodium methallylsulfonate) hollow fibers remained normoglycemic (70 days) for longer period of time in comparison to a control group (21 days). Moreover, the biocompatibility of the hydrogel proved to be satisfactory with no tissue necrosis and cellular inflammation during the syngeneic implantation period, though needs optimization for perfusion potential [152]. Smink et al. reported an efficient macrocapsule for islet transplantation using a prevasculared scaffold made of PDLLCL, which has a protective effect on the viability and function of the islets, though its efficiency was lower than that of transplanted islet under the kidney capsule. The PDLLCL porous scaffold had two polyethylene (PE) rods with high hydrophobicity. The scaffold was implanted subcutaneously in diabetic mice to allow for vascularization of the scaffold. After 4 weeks, the PE rods were removed from the scaffold and islets were transplanted inside the channels for 10 weeks [153]. The porous nature of this scaffold did not provide immuneprotection (Figure 2B).

Also other studies have focused on application of porous polymer scaffolds. These type of scaffolds were not intended to serve as an immune barrier. Rather, they were specifically designed to provide a support for islets for cellular infiltration and vascularization within the transplanted graft. Blomeier et al., transplanted murine 
islets using microporous scaffolds, which were fabricated from copolymers of lactide and glycolide (PLG) into intraperitoneal fat tissue of syngenic diabetic mice. They reported that the efficiency of the transplanted islets in the scaffold was similar to islets under the kidney capsule, but it was better than the group without scaffold. Moreover, they observed a preserved morphology of the islets transplanted by means of scaffold [154].

Recently a porous microwell scaffold for islet transplantation has been developed (Figure 2C). The advantage of this design is that the microwell structure prohibits islet aggregation and fusion, ensuring maintenance of the native rounded morphology and size of islets. When implanted in the epididymal fat tissue of diabetic mice the results revealed that the microwell scaffold supported the viability of islets and restored the systemic glycemic control, though significant reduction in $\beta$-cells mass was observed after transplantation $[155,156]$.

\section{- Microencapsulation}

Microencapsulation (Figure 2 D and E) is mainly directed to the elimination of life-long immunosuppressive medication after transplantation. To date, the main obstacle for microencapsulation reported is poor biocompatibility of the capsule material, inadequate immune isolation, and hypoxia due to failure of revascularization [157]. A second problem is the difficulty to retrieve microcapsules (microspheres) after transplantation in the case of an immune reaction or cellular adherence. To address these problems, the application of different materials such as alginate, polysulphone (PS), poly(ethylene glycol) (PEG) poly(vinyl alcohol), and dimethylamioethyl methacrylate-methyl methacrylate copolymer were examined for microencapsulation of islets. For instance, several investigations using different chemical composition of alginate based capsules which varied in [158, 159] purity [160, 161], size[162, 163], integrity, and mechanical properties [164, 165] were performed to improve islet transplantation. Making a balance between all these factors is essential to have an adequate encapsulation, molecular diffusion and prevention of immune reactions. 
Sun et al. demonstrated normal glycemic control in the long term by intraperitoneally transplantation of Porcine islets in alginate-polylysine-alginate microcapsules into spontaneously diabetic monkeys. They reported that strength of the capsular membrane and purity of the islets as well as the purity of the alginate were important factors for the systems biocompatibility [166]. Omar et al. reported successful differentiation, maturation, and proliferation of microencapsulated porcine neonatal pancreatic cell clusters in barium alginate after transplantation in diabetic mice. They observed normoglycemia in $81 \%$ of the transplanted mice up to 20 weeks [167]. De Vos et al. stated that different compositions of alginate microcapsules could influence the encapsulation process. The amount of incomplete encapsulation depends on the composition and dimension of the alginate-capsules. They indicated that the alginate with high guluronic acid content and a viscosity close to the filtration limit results in minimal numbers of inadequate encapsulation [158]. Moreover, it was reported that binding of poly-l-lysine (PLL) to guluronic acid in alginate could induce an immune reaction. No fibrotic response was observed for different kinds of alginate in the absence of a PLL membrane [167]. It was reported that the coating of Alginate-PLL capsules with polyacrylic acid [168] or Ca-alginate layers [169] could prohibit immune reactions by covering the inflammatory PLL and by providing a smooth surface (Figure 2E). It was demonstrated that the addition of ECM molecules to the microspheres such as alginate could improve islet function. Gibly et al. transplanted encapsulated human islets with porous Poly(lactide-co-glycolide) (PLG) to the intraperitoneal fat of immune-deficient mice. The PLG capsules were treated with Col4 or mixtures with human ECM molecules consisting of LN, Col4, and heparin sulfate proteoglycan. They indicated that addition of ECM molecules, specially Col4, to the PLG microcapsules could significantly enhance the insulin release comparing to nontreated ECM microcapsules [117]. Liacua, by transplantation of the encapsulated islets with alginate combined with different sequence of ECM molecules indicated the importance of RGD and PDSGR sequences of LN on improving the function and survival of islets [113]. 


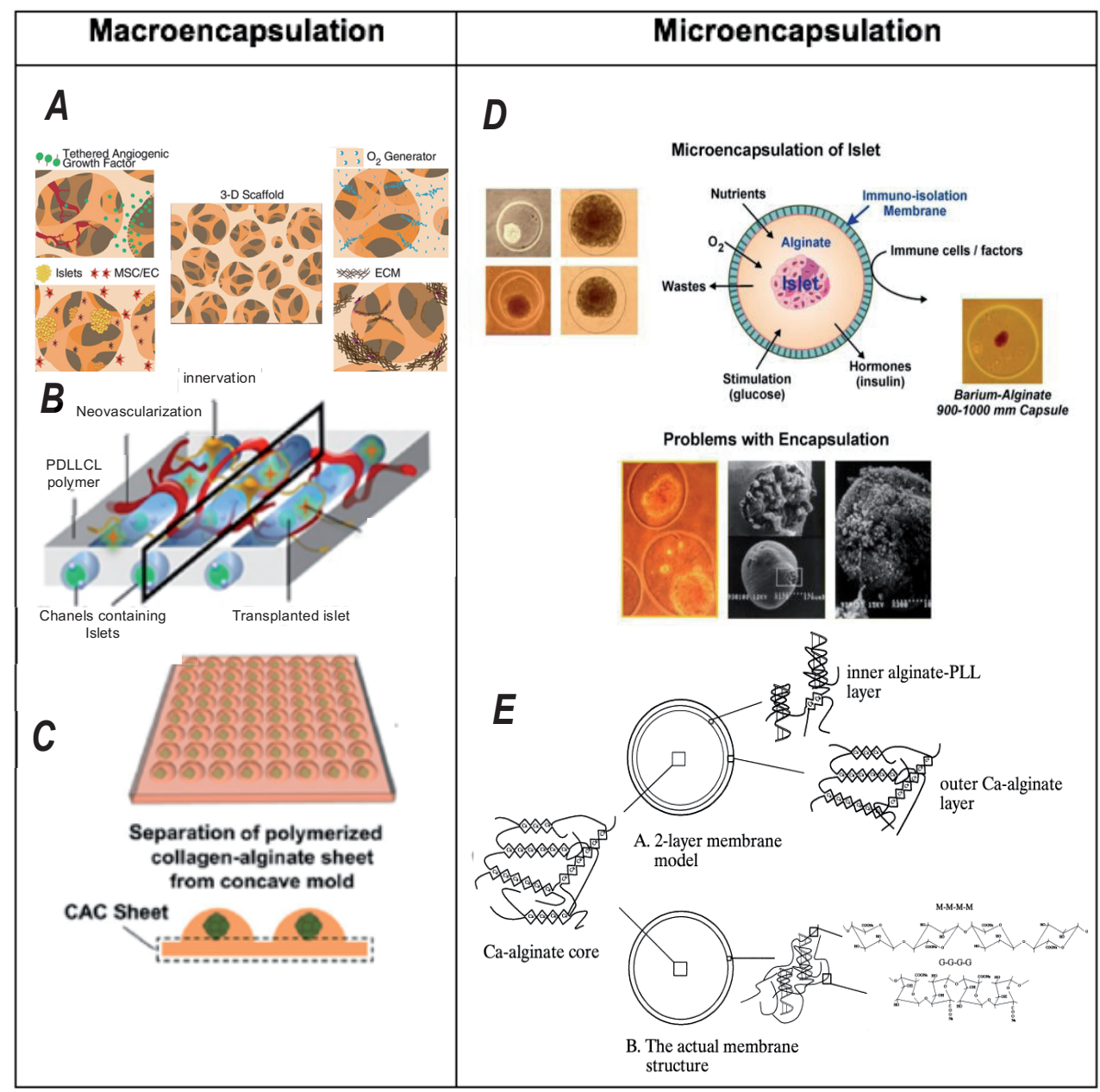

Figure 2. Schematic illustration of Marco- and Micro-encapsulation of islets. Adapted from[9, 170-173]. A) Macroporous scaffold including MSc, ECM, growth factors, $\mathrm{O}_{2}$ generator to improve the efficiency of scaffold for islet transplantation, B) Tubular prevascularized scaffold $\mathbf{C}$ ) Immunoprotective planar sheet scaffold D) Immunoprotective microencapsulation of islets. It allows for the transfer of insulin and wastes and nutrients while protecting from immune reaction. Problems with microencapsulation consiste of protrusion of islets which can result in immue reaction and graft failure by rupture of the capsules. E) Multilayer microcapsules.

\section{The dynamic niche: inflammation and scarring}

Autoimmunity causes Type 1 diabetes via a T-cell mediated destruction of $\beta$-cells [174]. The immune reaction is caused by genetic or environmental etiological factors such as viral infections, dietary factors, toxin, stressful life, etc. [175, 176]. 
In response to these factors, islets produce inflammatory mediators and upregulate the expression of the major histocompatibility complex class I (MCH-I). The major autoantigens that are involved in $\beta$-cell-specific autoimmunity are proinsulin, acid decarboxylase (GAD), tyrosine phosphatase-like protein, insulinoma antigen 2(IA2), phogrin (IA-2b), zinc transport 8, glutamic, and Carboxypeptidase H [177, 178]. The autoimmune reaction against $\beta$-cells starts by $T$ cells which escaped from the negative selection of the thymus. The $\beta$-cells release autoantigens that are taken up by antigen presenting cells (APC). This process initiates the diabetogenic response and leads to migration of T-cells into the circulation and initiates insulitis. The CD4+ cells recognize the autoantigens that are presented locally by $\beta$-cells. It is followed by stimulation of APC to secrete cytokines and nitric oxid (NO). The released cytokines and NO activate the $\mathrm{CD} 8+$. The CD8 + T-cells are differentiated to effector T-cells, which kill the $\beta$-cells $[177,179,180]$. As a consequence, the pancreas cannot perform its function regarding regulating blood glucose levels by secretion of insulin.

Whole pancreas or clinical islet transplantation of non-autologous origin - as possible treatments for type 1 diabetes - inherently activate immune responses of the body against the grafted tissue, which influences the efficacy of the treatments. In most cases islets can also immediately be destroyed due to cellular stress caused by the isolation process, which brings the islets in a pro-inflammatory state [181]. The pro-inflammatory cytokines activate different immune responses, which result in loss of islet mass [182]. The patients which have a successful islet transplantation do require systemic life-long administration of immunosuppressive drugs to prevent rejection. Patients usually suffer from side effects of these anti-rejection drugs, among which are increased susceptibility to infection, renal dysfunction, hyperlipidemia, anemia, mouth ulcers, and increased risk of cancer [183]. To prevail these problems short-term or local immunomodulators have been investigated. For instance, local inflammation could be controlled by engineering materials to release cytokines, such as transforming growth factor-b (TGFb) and IL10, to locally weaken the inflammatory responses [184]. However, such an 
immunomodulating strategy has often a short-term effect, for as long as the material releases the cytokine at pharmacologically relevant concentrations.

In order to provide long lasting immunosuppressive strategies, researchers have considered both macro- or microencapsulation strategies to protect the islets from the immune system. Macroencapsulation can trap a large quantity of islets in between a semi-permeable biocompatible barrier. By this technique both cellmediated and complement-mediated immune effectors are limited from contact with the transplanted islets. In addition, microencapsulation enables islets to be immuno isolated during transplantation. Therefore, patients do not need systemic administration of immunosuppression.

Alternatively, chemical modifications of materials have the potential to protect the transplanted islets from immune responses. For instance, Vegas et al. was able to decrease the inflammatory effect by means of chemically modified alginate hydrogels [185]. Overall, the generation of techniques to inhibit the host body's immune reaction can be further enhanced by efficient engineering to facilitate applications valuable to islet transplantation in diabetic patients.

\section{Vascular niche of islets}

Pancreatic islets are highly vascularized multicellular micro-organs. The islets receive $5-15 \%$ of total pancreatic blood flow in rats, $2-5 \%$ in mice and $15-20 \%$ in rabbits. Regarding humans there are still no methods to measure islet blood flow. Considering that the vessels around each islet have the essential function of providing oxygen and nutrient to islet cells and serve for transportation of the islet hormones, it is of particular importance to study vascularization of islets, it is influence on islets and the possibilities to imply this in an engineeringstrategy for islets.

The supply and diffusion of oxygen into tissues, varies in differnet tissues and even it might differ in various parts of a tissue. For instance, the surface oxygen 
tension in islets is $40 \mathrm{mmHg}$, which is 10 times higher than oxygen tension in the exocrine pancreas. Many studies emphasized on the importance of oxygen in order to have highly active metabolic cells [186, 187]. In healthy islets of Langerhans, the oxygen is provided through a dense, glomerular-like network of capillaries[187]. This highly dense network of microcapillaries ensures islets are provided with a high amount of oxygen, since even moderate levels of oxygen tension can result in loss of function [188]. Therefore, one of the important parameters to include in islet transplantation is to maximize oxygen transport into islets.

Continuously providing a sufficient amount of oxygenation following transplantation plays a crucial role in islet viability and function. It was shown that the oxygen tension in transplanted islets, regardless of their engraftment site, is lower than in native islets and leads to deficiency in glucose responsiveness or islet apoptosis [189]. To address the hypoxia problem, Ludwig et al., used a macrochamber, which was specifically engineered for islet transplantation. This chamber filled with encapsulated islets was implanted subcutaneously in diabetic rodents and oxygen diffusion was facilitated through a gas permeable membrane to supply islets with adequate levels of oxygen. Results demonstrated enhanced function of encapsulated islets, which was attributed to the oxygen supply as well as the pretreatment of islets with a growth hormone-releasing hormone agonist [190]. Alternatively, Jung et al. developed hypoxia resistant islets by transducing an antioxidant protein (metallothionein) into islets [191]. Subcutaneous transplantation of the metallothionein treated islets in mice resulted in similar glucose responsiveness to normal mice for 5 days [191]. Another approach to prevail hypoxia is to incorporate oxygen releasing materials such as PEGylated hemoglobin during islet engineering [192-194]. After all, there still there is no effective strategy to prevent islets hypoxia after transplantation and more studies in this area are necessary.

Besides the role of the dense vascular network around islets to prevent hypoxia and supply cells with nutrients, the endothelium senses and responds to blood 
glucose levels. The cell-cell interactions between $\beta$-cells and adjacent endothelial cells contributes to control the amount of insulin released. In addition, blood circulation affects $\beta$-cell proliferation, function and differentiation by adjacent ECM molecules and releasing growth factors [195-197]. Thus, researchers have generally focused on improving vascularization in the transplanted islet in order to address all the limitations caused by poor vascularity. For instance, some were considered to stimulate revascularization by means of different growth factors (e.g. VEGF) or hormones (e.g. prolactin) [198-200], prevascularization of scaffolds [153], and genetic overexpression of HIF1a [201].

Yin et al., developed VEGF conjugated alginate hydrogels to encapsulate islets. Subcutaneous implantation of these constructs resulted in promotion of neoangiogenesis. Consequently, the mice demonstrated better control over blood glucose levels [200]. Johansson et al. demonstrated that prolactin stimulates angiogenesis in endogenous islets and improves the transplantation outcome by increasing the density of the blood vessels. In this study, human islets were incubated in prolactin before transplanting under the renal capsule of C57BL/ 6 mice. A month after transplantation, the evaluation of the islets indicated enhanced revascularization as well as improved blood perfusion and oxygen tension, which increased transplantation success rate [199]. There are numerious other molecules (e.g. fibroblast growth factors) that are involved in the angiogenesis of the islets that can accelerate revascularization in islet transplants [202]. Therefore, it is important to consider vascularization-promoting growth factors in engineering the islet microenvironment.

Another approach to improve islet transplants is to utilize revascularized scaffolds. In this regard, Vlahos et al. created vascularized tissue implants by coating collagen cylinders with endothelial cells. Subcutaneous injection of islets in the endothelial coated module exhibited normoglycemia in diabetic mice within 14-21 days. The most interesting finding of this study was the ability of the module to integrate with the host's vasculature leading to revascularization of the 
transplanted islet [203]. However, the efficiency of this approach should be examined for longer period of time.

\section{Islet transplantation site}

The distinct microenvironments found within the various islet implantation sites strongly influences the vulnerability, function, and survival of transplanted islets. A suitable implantation site should provide an adequate oxygen tension and sufficient nutrients to support islet survival immediately after transplantation and allow for rapid vascularization of the implant afterwards. Moreover, the implantation site should prevent, or at least minimize, islet loss due to immune reactions. In addition, accessibility and minimal side effects of post transplantation are important factors. To date several implantation sites have been explored, including the liver, pancreas, kidney capsule, spleen, gastrointestinal wall, omentum, subcutaneous space, and bone marrow.

\section{- Liver}

The liver is currently the most common used site for clinical islet transplantation. However, it is associated with several complications such as bleeding, portal hypertension or portal vein thrombosis [204]. However, the risk of these complications can be decreased by improving islet purity and heparin supplementation prior to islet infusion [204]. Moreover, significant loss of islet mass was reported shortly after transplantation due to macrophage and Kupffer cell mediated inflammations, activation of hemostasis, thrombosis as well as the high levels of immunosuppressive drugs [205, 206].

\section{- Pancreas}

The pancreas, as the native microenvironment of islets, is also suggested as a suitable site for islet transplantation. Islet transplantation in the pancreases of the rat requires lower islet numbers in comparison to infusion in the portal vein. However, the invasive procedure with risk of inducing pancreatitis and 
autoimmunity of the diabetic patients may make it a poor site for islets transplantation [207].

\section{- Kidney subcapsule}

The kidney capsule is the most preferred transplantation site in experimental models usually in rodents due to the easy surgical procedure, retrievability by nephrectomy for histological and functional analyses, low mortality and absence of internal organ damaging. Although the oxygen tension in the kidney is lower than in the pancreas, about $12.5 \%$ to $25 \%$ of total islet mass could induce normoglycemia after transplantation under the kidney capsule of rats.

It was demonstrated that the low oxygen tension and blood flow does not seem to impair islet function in rodents [187]. Clinical islet transplantation into the kidney capsule of humans requires more islet mass compared to transplantation into the portal vein. The need for high numbers of islets as well as the invasive procedure for transplantation of islets into the kidney subcapsule may limit this site for clinical and experimental applications in human.

\section{- Spleen}

The rich vascular blood supply within the spleen is one of the major advantages of this site for transplantation, which is comparable to pancreatic parenchyma [208]. Intrasplenic islet transplantation indicated improvement in the function and preservation of the morphology of the islets [209, 210]. Islets transplanted in the spleen of a patient with type 1 diabetes resulted in normoglycemia without exogenous insulin for one year after transplantation. However, the increase in the number of lymphocytes and risk of bleeding in the spleen limits the application of this site for islet transplantation [211].

\section{- Gastrointestinal wall}

The gastrointestinal wall is an promising alternative implantation site for islet transplantation due to its dense vascular network, fast glucose sensing, and ease- 
of-access for surgical procedures [212]. Islet transplantation in gastric submucosa of mini pigs indicated better function compared to islets transplanted in the kidney subcapsule [213]. Moreover, islet transplantation in duodenal submucosal space of Syria golden hamsters indicated higher rates of revascularization within two weeks and lower rates of host inflammatory reactions [214, 215]. In addition, no sign of bleeding or inflammatory reaction was observed in the transplanted site in the pigs [215].

\section{- Omentum}

Omentum has a potential for islet transplantation because it is a well-vascularized site, has a good arterial pedicle, numerous lymphatic vessels and provides a large space for implantation [216, 217]. Moreover, it is suggested as an immuneprivileged site because of survival of the transplanted allogenic islets in the greater omentum of non-immunosuppressed guinea pigs. To achieve glycemic control in mice, the omental pouch was reported as a better site compared to kidney, liver and muscle sites [218]. However, a higher number of islets was required to achieve normoglycemia in comparison to the spleen site in a dog model [219]. In addition, it was suggested as a better site for transplantation of unpurified islets, which can increase the risk of coagulopathy and hypertension by transplantation into the portal vein [220]. More long-term studies are required with animal models to confirm the efficiency of this site for clinical application in islet transplantation.

\section{- Subcutaneous}

Despite of easy accessibility of subcutaneous sites for transplantation, poor oxygen tension, limited blood supply, and lack of early neovascularization may result in a poor outcome. The neovascularization in this site could improve the survival of islets [221]. Pepper et al. indicated that inducing the immune reaction in a controlled manner could increase the local neovascularization. Prior to islet transplantation at this site, a hollow catheter was subcutaneously implanted to induce neovascularization at the site. After removing the catheter, transplantation of islets resulted in normoglycemia in diabetic mice [222]. Moreover, it is reported 
that angiogenesis induction by applying growth factors in this site could improve the function and survival of transplanted islets [221]. Despite of sufficient results by transplantation of islets subcutaneously in rats, the investigation for primates and human are missing.

\section{- Bone marrow}

Bone marrow provides an easy accessible site for transplantation. It is an immuneprivileged site for islets by preservation of the islets survival without using immunesuppression in both diabetic and non-diabetic transplanted mice [223, 224].

\section{Conclusion}

There are a large number of investigations going on regarding possible methods for treatment of type 1 diabetes. For instance one of the most promising treatment methods is engineering of cell aggregates and its surrounding microenvironment. Research has focused on the improvement of the efficiency of this method for clinical applications. Although there are several parameters that one should consider for islet engineering, numerous promising results have been achieved in this field. The interaction of islet cells with the ECM has a significant influence on morphology, function, and viability of islets. The type of applied biomaterials to encapsulate islets can have direct effect on the behavior of cells. Having thorough control over the number of cells as well as the morphology of islet aggregates inside a scaffold are other important requirements for successful islet engineering. It is believed that ECM molecules may play an important role in developing these strategies. Another important aspect is the supply of oxygen. The islets' high demand for oxygen and nutrition which should be provided by a vascular system, via revascularization at the site of the transplant, is essential for a successful transplant outcome. The endocrine function of islets depends on the blood flow in and around their microenvironment to adequately sense changes in blood glucose concentrations and efficiently secrete the endocrine hormones. Based on the 
morphology of the engineered islets, different sites for transplantation could be selected, which will result in long-term efficiency of the transplant. Overall, engineered islet microenvironments could be a versatile platform to address many of the key challenges, which hinder nowadays the success of islet transplantation. 


\section{References}

1. Atkinson, M.A. and Maclaren, N.K. (1994) The Pathogenesis of Insulin-Dependent Diabetes Mellitus. New England Journal of Medicine 331 (21), 1428-1436.

2. Roglic, G. (2016) WHO Global report on diabetes: A summary. International Journal of Noncommunicable Diseases 1 (1), 3.

3. Broadbent, E. et al. (2011) Illness and treatment perceptions are associated with adherence to medications, diet, and exercise in diabetic patients. Diabetes care 34 (2), 338-340.

4. Muñana, K.R. (1995) Long-term complications of diabetes mellitus, Part I: Retinopathy, nephropathy, neuropathy. Veterinary Clinics of North America: Small Animal Practice 25 (3), 715-730.

5. Sutherland, D.E. et al. (2001) Pancreas transplantation for treatment of diabetes mellitus. World journal of surgery 25 (4), 487-496.

6. Medarova, Z. and Moore, A. (2008) Non-invasive detection of transplanted pancreatic islets. Diabetes, Obesity and Metabolism 10 (s4), 88-97.

7. Cross, S.E. et al. (2008) Collagenase penetrates human pancreatic islets following standard intraductal administration. Transplantation 86 (7), 907-911.

8. Amer, L.D. et al. (2014) Tissue engineering approaches to cell-based type 1 diabetes therapy. Tissue Engineering Part B: Reviews 20 (5), 455-467.

9. Coronel, M.M. and Stabler, C.L. (2013) Engineering a local microenvironment for pancreatic islet replacement. Current opinion in biotechnology 24 (5), 900-908. (C)2013 Coronel, M.M. and Stabler, C.L.. Available from: https://doi.org/10.1016/j.copbio.2013.05.004

10. Kaviani, M. and Azarpira, N. (2016) Insight into microenvironment remodeling in pancreatic endocrine tissue engineering: Biological and biomaterial approaches. Tissue Engineering and Regenerative Medicine 13 (5), 475-484.

11. Stendahl, J.C. et al. (2009) Extracellular matrix in pancreatic islets: relevance to scaffold design and transplantation. Cell transplantation 18 (1), 1-12.

12. Lemos, N.E. et al. (2017) Use of additives, scaffolds and extracellular matrix components for improvement of human pancreatic islet outcomes in vitro: A systematic review. Islets, e1335842.

13. Olerud, J. et al. (2009) Vascular niche of pancreatic islets. Expert Review of Endocrinology \& Metabolism 4 (5), 481-491.

14. Bonner-Weir, S. (1991) Anatomy of the islet of Langerhans. The endocrine pancreas, 1527.

15. Edlund, H. (2002) Pancreatic organogenesis-developmental mechanisms and implications for therapy. Nature Reviews Genetics 3 (7), 524-532.

16. Kim, A. et al. (2009) Islet architecture: A comparative study. Islets 1 (2), 129-36.

17. Cabrera, O. et al. (2006) The unique cytoarchitecture of human pancreatic islets has implications for islet cell function. Proceedings of the National Academy of Sciences of the United States of America 103 (7), 2334-2339. 
18. Brissova, M. et al. (2005) Assessment of human pancreatic islet architecture and composition by laser scanning confocal microscopy. Journal of Histochemistry \& Cytochemistry 53 (9), 1087-1097.

19. Brissova, M. et al. (2005) Assessment of human pancreatic islet architecture and composition by laser scanning confocal microscopy. J Histochem Cytochem 53 (9), 1087-97.

20. Orci, L. et al. (1976) Hypertrophy and hyperplasia of somatostatin-containing D-cells in diabetes. Proc Natl Acad Sci U S A 73 (4), 1338-42.

21. Gersell, D.J. et al. (1979) Regional distribution and concentration of pancreatic polypeptide in the human and canine pancreas. Diabetes 28 (1), 11-5.

22. Thowfeequ, S. et al. (2007) Transdifferentiation in developmental biology, disease, and in therapy. Developmental Dynamics 236 (12), 3208-3217.

23. Horb, M.E. et al. (2003) Experimental conversion of liver to pancreas. Current Biology 13 (2), 105-115.

24. Hui, H. et al. (2001) Glucagon-like peptide 1 induces differentiation of islet duodenal homeobox-1-positive pancreatic ductal cells into insulin-secreting cells. Diabetes 50 (4), 785796.

25. Porat, S. and Dor, Y. (2007) New sources of pancreatic beta cells. Current diabetes reports 7 (4), 304-308.

26. Xu, X. et al. (2008) $\beta$ cells can be generated from endogenous progenitors in injured adult mouse pancreas. Cell 132 (2), 197-207.

27. Piper, K. et al. (2004) Beta cell differentiation during early human pancreas development. Journal of Endocrinology 181 (1), 11-23.

28. Baeyens, L. et al. (2014) Transient cytokine treatment induces acinar cell reprogramming and regenerates functional beta cell mass in diabetic mice. Nature biotechnology 32 (1), 76-83.

29. Puri, S. and Hebrok, M. (2010) Cellular plasticity within the pancreas-lessons learned from development. Developmental cell 18 (3), 342-356.

30. Collombat, P. et al. (2003) Opposing actions of Arx and Pax4 in endocrine pancreas development. Genes \& development 17 (20), 2591-2603.

31. Biressi, S. et al. (2008) The homeobox gene Arx is a novel positive regulator of embryonic myogenesis. Cell Death \& Differentiation 15 (1), 94-104.

32. Collombat, P. et al. (2009) The ectopic expression of Pax4 in the mouse pancreas converts progenitor cells into $a$ and subsequently $\beta$ cells. Cell 138 (3), 449-462.

33. Schaffer, A.E. et al. (2013) Nkx6. 1 controls a gene regulatory network required for establishing and maintaining pancreatic Beta cell identity. PLoS Genet 9 (1), e1003274.

34. Seaberg, R.M. et al. (2004) Clonal identification of multipotent precursors from adult mouse pancreas that generate neural and pancreatic lineages. Nature biotechnology 22 (9), 11151124.

35. Smukler, S.R. et al. (2011) The adult mouse and human pancreas contain rare multipotent stem cells that express insulin. Cell stem cell 8 (3), 281-293. 
36. Assady, S. et al. (2001) Insulin production by human embryonic stem cells. Diabetes 50 (8), 1691-1697.

37. D'Amour, K.A. et al. (2005) Efficient differentiation of human embryonic stem cells to definitive endoderm. Nature biotechnology 23 (12), 1534.

38. D'Amour, K.A. et al. (2006) Production of pancreatic hormone-expressing endocrine cells from human embryonic stem cells. Nature biotechnology 24 (11), 1392.

39. Kroon, E. et al. (2008) Pancreatic endoderm derived from human embryonic stem cells generates glucose-responsive insulin-secreting cells in vivo. Nature biotechnology 26 (4), 443.

40. Nostro, M.C. et al. (2011) Stage-specific signaling through TGF $\beta$ family members and WNT regulates patterning and pancreatic specification of human pluripotent stem cells. Development 138 (5), 861-871.

41. Cheng, X. et al. (2012) Self-renewing endodermal progenitor lines generated from human pluripotent stem cells. Cell stem cell 10 (4), 371-384.

42. Kelly, C. et al. (2011) Role of islet structure and cellular interactions in the control of insulin secretion. Islets 3 (2), 41-47.

43. Ichihara, Y. et al. (2016) Size effect of engineered islets prepared using microfabricated wells on islet cell function and arrangement. Heliyon 2 (6), e00129.

44. Jiang, G. and Zhang, B.B. (2003) Glucagon and regulation of glucose metabolism. American Journal of Physiology-Endocrinology And Metabolism 284 (4), E671-E678.

45. Cabrera, O. et al. (2008) Glutamate is a positive autocrine signal for glucagon release. Cell metabolism 7 (6), 545-554.

46. Gaisano, H. et al. (2012) Glucagon secretion and signaling in the development of diabetes. Frontiers in physiology 3, 349. (C) 2012 Gaisano, MacDonald and Vranic. Available from: https://doi.org/10.3389/fphys.2012.00349

47. Xu, E. et al. (2006) Intra-islet insulin suppresses glucagon release via GABA-GABA A receptor system. Cell metabolism 3 (1), 47-58.

48. Wendt, A. et al. (2004) Glucose inhibition of glucagon secretion from rat a-cells is mediated by GABA released from neighboring $\beta$-cells. Diabetes 53 (4), 1038-1045.

49. Rorsman, P. et al. (1989) Glucose-inhibition of glucagon secretion involves activation of GABAA-receptor chloride channels.

50. Ishihara, $H$. et al. (2003) Islet $\beta$-cell secretion determines glucagon release from neighbouring a-cells. Nature cell biology 5 (4), 330-335.

51. Hansen, A. et al. (2011) Upregulation of alpha cell glucagon-like peptide 1 (GLP-1) in Psammomys obesus - an adaptive response to hyperglycaemia? Diabetologia 54 (6), 13791387.

52. Ellingsgaard, $H$. et al. (2011) Interleukin-6 enhances insulin secretion by increasing glucagon-like peptide-1 secretion from L cells and alpha cells. Nature medicine 17 (11), 14811489.

53. Kjems, L.L. et al. (2003) The influence of GLP-1 on glucose-stimulated insulin secretion. Diabetes 52 (2), 380-386. 
54. Farilla, L. et al. (2003) Glucagon-like peptide 1 inhibits cell apoptosis and improves glucose responsiveness of freshly isolated human islets. Endocrinology 144 (12), 5149-5158.

55. Kojima, N. et al., Engineering of pseudoislets: effect on insulin secretion activity by cell number, cell population, and microchannel networks, Transplantation proceedings, Elsevier, 2014, pp. 1161-1165.

56. Cejvan, $K$. et al. (2003) Intra-islet somatostatin regulates glucagon release via type 2 somatostatin receptors in rats. Diabetes 52 (5), 1176-1181.

57. Bonner-Weir, S. and Orci, L. (1982) New perspectives on the microvasculature of the islets of Langerhans in the rat. Diabetes 31 (10), 883-889.

58. Schwetz, T.A. et al. (2013) Neuropeptide $Y$ and somatostatin inhibit insulin secretion through different mechanisms. American Journal of Physiology-Endocrinology and Metabolism 304 (2), E211-E221.

59. Schuit, F. et al. (1989) Sensitivity of rat pancreatic $A$ and $B$ cells to somatostatin. Diabetologia 32 (3), 207-212.

60. Ramachandran, K. et al. (2012) Engineering islets for improved performance by optimized reaggregation in a micromold. Tissue Engineering Part A 19 (5-6), 604-612.

61. Heller, R.S. et al. (2005) Genetic determinants of pancreatic e-cell development. Developmental biology 286 (1), 217-224.

62. Dezaki, K. et al. (2008) Ghrelin is a physiological regulator of insulin release in pancreatic islets and glucose homeostasis. Pharmacology \& therapeutics 118 (2), 239-249.

63. Kageyama, $H$. et al. (2005) Morphological analysis of ghrelin and its receptor distribution in the rat pancreas. Regulatory peptides 126 (1), 67-71.

64. Dezaki, K. et al. (2006) Blockade of pancreatic islet-derived ghrelin enhances insulin secretion to prevent high-fat diet-induced glucose intolerance. Diabetes 55 (12), 3486-3493.

65. Nittala, A. et al. (2007) Investigating the role of islet cytoarchitecture in its oscillation using a new $\beta$-cell cluster model. PLoS One 2 (10), e983.

66. Nam, K.-H. et al. (2010) Size-based separation and collection of mouse pancreatic islets for functional analysis. Biomedical microdevices 12 (5), 865-874.

67. Wojtusciszyn, A. et al. (2008) Insulin secretion from human beta cells is heterogeneous and dependent on cell-to-cell contacts. Diabetologia 51 (10), 1843.

68. O'sullivan, E. et al. (2010) Rat islet cell aggregates are superior to islets for transplantation in microcapsules. Diabetologia 53 (5), 937-945.

69. Halban, P.A. et al. (1982) The possible importance of contact between pancreatic islet cells for the control of insulin release. Endocrinology 111 (1), 86-94.

70. Krishnan, R. et al. (2016) Strategies to Combat Hypoxia in Encapsulated Islet Transplantation. Surgery: Current Research 6 (2), 1-10.

71. MacGregor, R. et al. (2006) Small rat islets are superior to large islets in in vitro function and in transplantation outcomes. American Journal of Physiology-Endocrinology and Metabolism 290 (5), E771-E779. 
72. Chou, H.-F. and Ipp, E. (1990) Pulsatile insulin secretion in isolated rat islets. Diabetes 39 (1), 112-117.

73. Cirulli, V. (2015) Cadherins in Islet $\beta$-Cells: More Than Meets the Eye. Diabetes 64 (3), $709-$ 711 .

74. Penko, D. et al. (2015) Endothelial progenitor cells enhance islet engraftment, influence $\beta$ cell function, and modulate islet connexin 36 expression. Cell transplantation 24 (1), 37-48.

75. Konstantinova, I. et al. (2007) EphA-Ephrin-A-mediated $\beta$ cell communication regulates insulin secretion from pancreatic islets. Cell 129 (2), 359-370.

76. Eberhard, D. and Lammert, E. (2009) The pancreatic $\beta$-cell in the islet and organ community. Current opinion in genetics \& development 19 (5), 469-475.

77. Dahl, U. et al. (1996) Cadherins regulate aggregation of pancreatic beta-cells in vivo. Development 122 (9), 2895-2902.

78. Jaques, F. et al. (2008) Dual effect of cell-cell contact disruption on cytosolic calcium and insulin secretion. Endocrinology 149 (5), 2494-2505.

79. Rogers, G.J. et al. (2007) E-cadherin and cell adhesion: a role in architecture and function in the pancreatic islet. Cellular Physiology and Biochemistry 20 (6), 987-994.

80. Esni, F. et al. (1999) Neural cell adhesion molecule (N-CAM) is required for cell type segregation and normal ultrastructure in pancreatic islets. The Journal of cell biology 144 (2), 325-337.

81. Olofsson, C.S. et al. (2009) Impaired insulin exocytosis in neural cell adhesion molecule-/mice due to defective reorganization of the submembrane F-actin network. Endocrinology 150 (7), 3067-3075.

82. Rocheleau, J.V. et al. (2006) Critical role of gap junction coupled KATP channel activity for regulated insulin secretion. PLoS biology 4 (2), e26.

83. Bavamian, S. et al. (2007) Islet-cell-to-cell communication as basis for normal insulin secretion. Diabetes, Obesity and Metabolism 9 (s2), 118-132.

84. Migliorini, A. et al. (2014) Islet cell plasticity and regeneration. Molecular metabolism 3 (3),268-274. (C)2011 Migliorini, A. et al. Available from:

https://doi.org/10.1016/j.molmet.2014.01.010

85. Jiang, F.-X. and Morahan, G. (2011) Pancreatic Stem Cells: Unresolved Business. In Stem Cells in Clinic and Research, InTech. (C) 2011 Jiang FX, Morahan G. Published in [short citation] under CC BY-NC-SA 3.0 license. Available from: http://dx.doi.org/10.5772/23760.

86. Michel, G. et al. (2010) The cell wall polysaccharide metabolism of the brown alga Ectocarpus siliculosus. Insights into the evolution of extracellular matrix polysaccharides in Eukaryotes. New Phytologist 188 (1), 82-97.

87. Abedin, M. and King, N. (2010) Diverse evolutionary paths to cell adhesion. Trends in cell biology 20 (12), 734-742.

88. Bogdani, M. et al. (2014) Extracellular matrix components in the pathogenesis of type 1 diabetes. Current diabetes reports 14 (12), 552.

89. Deijnen, J. et al. (1994) Distribution of collagens type I, type III and type V in the pancreas of rat, dog, pig and man. Cell and tissue research 277 (1), 115-121. 
90. Korpos, É. et al. (2013) The peri-islet basement membrane, a barrier to infiltrating leukocytes in type 1 diabetes in mouse and human. Diabetes 62 (2), 531-542.

91. Virtanen, I. et al. (2008) Blood vessels of human islets of Langerhans are surrounded by a double basement membrane. Diabetologia 51 (7), 1181-1191.

92. Singh, P. et al. (2010) Assembly of fibronectin extracellular matrix. Annual review of cell and developmental biology 26, 397-419.

93. Wang, R. et al. (2005) Fibronectin's central cell-binding domain supports focal adhesion formation and Rho signal transduction. Journal of Biological Chemistry 280 (31), 2880328810.

94. Koivunen, E. et al. (1994) [17] Peptides in cell adhesion research. Methods in enzymology 245, 346-369.

95. Cirulli, V. et al. (2000) Expression and Function of av $\beta 3$ and av $\beta 5$ Integrins in the Developing Pancreas. The Journal of cell biology 150 (6), 1445-1460.

96. Kaido, T. et al. (2004) av-Integrin utilization in human $\beta$-cell adhesion, spreading, and motility. Journal of Biological Chemistry 279 (17), 17731-17737.

97. Pinkse, G.G. et al. (2006) Integrin signaling via RGD peptides and anti- $\beta 1$ antibodies confers resistance to apoptosis in islets of Langerhans. Diabetes 55 (2), 312-317.

98. Wang, R. and Rosenberg, L. (1999) Maintenance of beta-cell function and survival following islet isolation requires re-establishment of the islet-matrix relationship. Journal of Endocrinology 163 (2), 181-190.

99. Edamura, K. et al. (2003) BRIEF COMMUNICATION: Effect of Adhesion or Collagen Molecules on Cell Attachment, Insulin Secretion, and Glucose Responsiveness in the Cultured Adult Porcine Endocrine Pancreas: A Preliminary Study. Cell transplantation 12 (4), 439-446.

100. Cheng, J.Y. et al. (2011) Matrix components and scaffolds for sustained islet function. Tissue Engineering Part B: Reviews 17 (4), 235-247.

101. Jiang, F.-X. et al. (2001) Regulation of laminin 1-induced pancreatic beta-cell differentiation by alpha6 integrin and alpha-dystroglycan. Molecular Medicine 7 (2), 107.

102. Kaido, T. et al. (2004) Regulation of human $\beta$-cell adhesion, motility, and insulin secretion by collagen IV and its receptor a1ß1. Journal of Biological Chemistry 279 (51), 53762-53769.

103. Hopcroft, D. et al. (1985) Structure-function relationships in pancreatic islets: support for intraislet modulation of insulin secretion. Endocrinology 117 (5), 2073-2080.

104. Heino, J. (2003) Cellular signaling by collagen-binding integrins. I Domains in integrins. Georgetown, TX: Landes Bioscience, 143-152.

105. Hughes, S.J. et al. (2006) Characterisation of collagen VI within the islet-exocrine interface of the human pancreas: implications for clinical islet isolation? Transplantation 81 (3), 423426.

106. Bonner-Weir, S. (1988) Morphological evidence for pancreatic polarity of $\beta$-cell within islets of Langerhans. Diabetes 37 (5), 616-621.

107. Kaido, T. et al. (2006) Impact of defined matrix interactions on insulin production by cultured human $\beta$-cells. Diabetes 55 (10), 2723-2729. 
108. Hamaia, S. and Farndale, R.W. (2014) Integrin recognition motifs in the human collagens. In I Domain Integrins, pp. 127-142, Springer.

109. Wang, R. et al. (2005) Role for $\beta 1$ integrin and its associated a3, a5, and a6 subunits in development of the human fetal pancreas. Diabetes 54 (7), 2080-2089.

110. Salvay, D.M. et al. (2008) Extracellular matrix protein-coated scaffolds promote the reversal of diabetes after extrahepatic islet transplantation. Transplantation 85 (10), 1456.

111. Hadley, J.C. et al. (1998) Glycation changes the charge distribution of type I collagen fibrils. Glycoconjugate journal 15 (8), 835-840.

112. Olsen, B. (1993) Basement membrane collagens (type IV). Guidebook to the Extracellular Matrix and Adhesion Proteins.

113. Llacua, A. et al. (2016) Extracellular matrix components supporting human islet function in alginate-based immunoprotective microcapsules for treatment of diabetes. Journal of Biomedical Materials Research Part A 104 (7), 1788-1796.

114. Nagata, N. et al. (2001) Evaluation of insulin secretion of isolated rat islets cultured in extracellular matrix. Cell transplantation 10 (4-1), 447-451.

115. Nagata, N. et al. (2002) Co-culture of extracellular matrix suppresses the cell death of rat pancreatic islets. Journal of Biomaterials Science, Polymer Edition 13 (5), 579-590.

116. Yap, W.T. et al. (2013) Collagen IV-modified scaffolds improve islet survival and function and reduce time to euglycemia. Tissue Engineering Part A 19 (21-22), 2361-2372.

117. Gibly, R.F. et al. (2013) Porous scaffolds support extrahepatic human islet transplantation, engraftment, and function in mice. Cell transplantation 22 (5), 811-819.

118. Colognato, H. and Yurchenco, P.D. (2000) Form and function: the laminin family of heterotrimers. Developmental Dynamics 218 (2), 213-234.

119. Jiang, F.-X. et al. (2002) Distinct distribution of laminin and its integrin receptors in the pancreas. Journal of Histochemistry \& Cytochemistry 50 (12), 1625-1632.

120. Irving-Rodgers, $H$. et al. (2008) Molecular composition of the peri-islet basement membrane in NOD mice: a barrier against destructive insulitis. Diabetologia 51 (9), 1680-1688.

121. Otonkoski, T. et al. (2008) Unique basement membrane structure of human pancreatic islets: implications for $\beta$-cell growth and differentiation. Diabetes, obesity and metabolism 10 (s4), 119-127.

122. Nikolova, G. et al. (2006) The vascular basement membrane: a niche for insulin gene expression and $\beta$ cell proliferation. Developmental cell 10 (3), 397-405.

123. Jiang, F.-X. et al. (1999) Laminin-1 promotes differentiation of fetal mouse pancreatic beta-cells. Diabetes 48 (4), 722-730.

124. Banerjee, M. et al. (2012) Proliferation and plasticity of human beta cells on physiologically occurring laminin isoforms. Molecular and cellular endocrinology 355 (1), 7886.

125. Parnaud, G. et al. (2006) Blockade of $\beta 1$ integrin-laminin-5 interaction affects spreading and insulin secretion of rat $\beta$-cells attached on extracellular matrix. Diabetes 55 (5), 14131420 . 
126. Brandhorst, D. et al. (2015) Co-culture with extracellular matrix proteins reduces hypoxiainduced human islet cell death. Xenotransplantation 22, S192.

127. Belkin, A.M. and Stepp, M.A. (2000) Integrins as receptors for laminins. Microscopy research and technique 51 (3), 280-301.

128. Graf, J. et al. (1987) Identification of an amino acid sequence in laminin mediating cell attachment, chemotaxis, and receptor binding. Cell 48 (6), 989-996.

129. Kleinman, H.K. et al. (1989) Identification of a second active site in laminin for promotion of cell adhesion and migration and inhibition of in vivo melanoma lung colonization. Archives of biochemistry and biophysics 272 (1), 39-45.

130. Kleinman, $H$. et al. (1988) Laminin receptors for neurite formation. Proceedings of the National Academy of Sciences 85 (4), 1282-1286.

131. TASHIRO, K.-i. et al. (1999) An IKLLI-containing peptide derived from the laminin a1 chain mediating heparin-binding, cell adhesion, neurite outgrowth and proliferation, represents a binding site for integrin a3 $\beta 1$ and heparan sulphate proteoglycan. Biochemical Journal 340 (1), 119-126.

132. Nomizu, M. et al. (1998) Cell binding sequences in mouse laminin a1 chain. Journal of Biological Chemistry 273 (49), 32491-32499.

133. Parsons, S.F. et al. (2001) Lutheran blood group glycoprotein and its newly characterized mouse homologue specifically bind a5 chain-containing human laminin with high affinity. Blood 97 (1), 312-320.

134. Lammert, E. and Kragl, M. (2015) Basement Membrane in Pancreatic Islet Function. In Islets of Langerhans, pp. 39-58, Springer.

135. Felding-Habermann, B. and Cheresh, D.A. (1993) Vitronectin and its receptors. Current opinion in cell biology 5 (5), 864-868.

136. Høgåsen, K. et al. (1992) Heparin-binding properties of vitronectin are linked to complex formation as illustrated by in vitro polymerization and binding to the terminal complement complex. Journal of Biological Chemistry 267 (32), 23076-23082.

137. Kreis, T. and Vale, R. (1999) Guidebook to the extracellular matrix, anchor, and adhesion proteins, Sambrook and Tooze Publications.

138. Song, J.J. and Ott, H.C. (2011) Organ engineering based on decellularized matrix scaffolds. Trends in molecular medicine 17 (8), 424-432.

139. Orlando, G. et al. (2012) Production and implantation of renal extracellular matrix scaffolds from porcine kidneys as a platform for renal bioengineering investigations. Annals of surgery 256 (2), 363-370.

140. Wang, $Y$. et al. (2011) Lineage restriction of human hepatic stem cells to mature fates is made efficient by tissue-specific biomatrix scaffolds. Hepatology 53 (1), 293-305.

141. De Carlo, E. et al. (2010) Pancreatic acellular matrix supports islet survival and function in a synthetic tubular device: in vitro and in vivo studies. International journal of molecular medicine 25 (2), 195-202.

142. Conrad, C. et al. (2010) Bio-engineered endocrine pancreas based on decellularized pancreatic matrix and mesenchymal stem cell/islet cell coculture. Journal of the American College of Surgeons 211 (3), S62. 
143. Mirmalek-Sani, S.-H. et al. (2013) Porcine pancreas extracellular matrix as a platform for endocrine pancreas bioengineering. Biomaterials 34 (22), 5488-5495.

144. Hynes, R.O. (2009) The extracellular matrix: not just pretty fibrils. Science 326 (5957), 1216-1219.

145. Smink, A.M. et al. (2016) Selection of polymers for application in scaffolds applicable for human pancreatic islet transplantation. Biomedical Materials 11 (3), 035006.

146. Lacy, P.E. et al. (1991) Maintenance of normoglycemia in diabetic mice by subcutaneous xenografts of encapsulated islets. Science 254 (5039), 1782-1784.

147. Lanza, R. et al., Successful bovine islet xenografts in rodents and dogs using injectable microreactors, Transplantation proceedings, 1995, pp. 3211-3211.

148. Lanza, R.P. et al. (1992) Transplantation of encapsulated canine islets into spontaneously diabetic BB/Wor rats without immunosuppression. Endocrinology 131 (2), 637-642.

149. Storrs, R. et al. (2001) Preclinical development of the Islet Sheet. Annals of the New York Academy of Sciences 944 (1), 252-266.

150. Lanza, R.P. et al. (1994) XENOGENEIC HUMORAL RESPONSES TO ISLETS TRANSPLANTED IN BIOHYBRID DIFFUSION CHAMBERS. Transplantation 57 (9), 1371-1375.

151. Lanza, R.P. et al. (1992) Treatment of severely diabetic pancreatectomized dogs using a diffusion-based hybrid pancreas. Diabetes 41 (7), 886-889.

152. Prevost, P. et al. (1997) Application of AN69® hydrogel to islet encapsulation. Annals of the New York Academy of Sciences 831 (1), 344-349.

153. Smink, A.M. et al. (2017) The Efficacy of a Prevascularized, Retrievable Poly (D, L,-lactideco-[epsilon]-caprolactone) Subcutaneous Scaffold as Transplantation Site for Pancreatic Islets. Transplantation.

154. Blomeier, H. et al. (2006) Polymer scaffolds as synthetic microenvironments for extrahepatic islet transplantation. Transplantation 82 (4), 452.

155. Buitinga, M. et al. (2017) Micro-fabricated scaffolds lead to efficient remission of diabetes in mice. Biomaterials 135, 10-22.

156. Buitinga, M. et al. (2013) Microwell scaffolds for the extrahepatic transplantation of islets of Langerhans. PloS one 8 (5), e64772.

157. Vaithilingam, V. and Tuch, B.E. (2011) Islet transplantation and encapsulation: an update on recent developments. The review of diabetic studies: RDS 8 (1), 51.

158. De Vos, P. et al. (1996) Factors influencing the adequacy of microencapsulation of rat pancreatic islets1. Transplantation 62 (7), 888-893.

159. De Vos, P. et al., Is it possible to use the standard alginate-PLL procedure for production of small capsules?, Transplantation proceedings, Elsevier, 1998, pp. 492-493.

160. De Vos, P. et al. (1997) Improved biocompatibility but limited graft survival after purification of alginate for microencapsulation of pancreatic islets. Diabetologia 40 (3), 262270 .

161. Cochrum, K. et al., Successful xenografts in mice with microencapsulated rat and dog islets, Transplantation proceedings, 1995, p. 3297. 
162. De Vos, P. et al. (1996) Association between capsule diameter, adequacy of encapsulation, and survival of microencapsulated rat islet allografts1. Transplantation 62 (7), 893-899.

163. Chicheportiche, D. and Reach, G. (1988) In vitro kinetics of insulin release by microencapsulated rat islets: effect of the size of the microcapsules. Diabetologia 31 (1), 54-57.

164. De Vos, P. et al., Possible relationship between fibrotic overgrowth of alginate-polylysinealginate microencapsulated pancreatic islets and the microcapsule integrity, Transplantation proceedings, 1994, pp. 782-783.

165. Lanza, R.P. et al. (1999) Xenotransplantation of cells using biodegradable microcapsules. Transplantation 67 (8), 1105-1111.

166. Sun, Y. et al. (1996) Normalization of diabetes in spontaneously diabetic cynomologus monkeys by xenografts of microencapsulated porcine islets without immunosuppression. Journal of Clinical Investigation 98 (6), 1417.

167. Omer, A. et al. (2003) Survival and maturation of microencapsulated porcine neonatal pancreatic cell clusters transplanted into immunocompetent diabetic mice. Diabetes 52 (1), 6975 .

168. Bünger, C. et al. (2003) Biocompatibility and surface structure of chemically modified immunoisolating alginate-PLL capsules. Journal of Biomedical Materials Research Part A 67 (4), 1219-1227.

169. de Vos, P. et al. (2003) Long-term biocompatibility, chemistry, and function of microencapsulated pancreatic islets. Biomaterials 24 (2), 305-312.

170. Lee, B.R. et al. (2012) In situ formation and collagen-alginate composite encapsulation of pancreatic islet spheroids. Biomaterials 33 (3), 837-845. (C)2012 Lee, B.R. et al. Available from: https://doi.org/10.1016/j.biomaterials.2011.10.014

171. Mihalicz, D. et al. (2011) Porcine Islet Xenotransplantation for the treatment of Type 1 diabetes. In Type 1 Diabetes-Pathogenesis, Genetics and Immunotherapy, InTech. (C) 2011 Mihalicz D, Rajotte RV, Rayat GR. Published in [short citation] under CC BY 3.0 license. Available from: http://dx.doi.org/10.5772/22206.

172. Alexander, M. et al. (2017) Avoiding Immunosuppression for Islet Transplantation: Use of Protective Biomaterials. In Challenges in Pancreatic Pathology, InTech. (C) 2017 Alexander $M$, Nguyen H, Flores A, Li S, De Vos P, Botvinick E, Lakey J. Published in [short citation] under CC BY 3.0 license. Available from: http://dx.doi.org/10.5772/66492.

173. de Vos, P. et al. (2009) Multiscale requirements for bioencapsulation in medicine and biotechnology. Biomaterials 30 (13), 2559-2570. (C)2009 de Vos, P. et al. Available from: https://doi.org/10.1016/j.biomaterials.2009.01.014

174. Homo-Delarche, F. and Boitard, C. (1996) Autoimmune diabetes: the role of the islets of Langerhans. Immunology today 17 (10), 456-460.

175. Akerblom, H.K. et al. (2002) Environmental factors in the etiology of type 1 diabetes. American journal of medical genetics 115 (1), 18-29.

176. Askenasy, N. (2016) Mechanisms of diabetic autoimmunity: II-Is diabetes a central or peripheral disorder of effector and regulatory cells? Immunologic research 64 (1), 36-43. 
177. Thrower, S. and Bingley, P. (2010) What is type 1 diabetes? Medicine 38 (11), 592-596.

178. Roep, B.O. and Peakman, M. (2011) Diabetogenic T lymphocytes in human type 1 diabetes. Current opinion in immunology 23 (6), 746-753.

179. Lehuen, A. et al. (2010) Immune cell crosstalk in type 1 diabetes. Nature Reviews Immunology 10 (7), 501-513.

180. Graham, K.L. et al. (2012) Pathogenic mechanisms in type 1 diabetes: the islet is both target and driver of disease. Rev Diabet Stud 9 (4), 148-168.

181. Bottino, R. et al. (2004) Response of human islets to isolation stress and the effect of antioxidant treatment. Diabetes 53 (10), 2559-2568.

182. Azzi, J. et al. (2010) Immunological aspects of pancreatic islet cell transplantation. Expert review of clinical immunology 6 (1), 111-124.

183. Gruessner, R., Immunosuppression after pancreas transplantation, Transplantation proceedings, Elsevier, 2005, pp. 1663-1666.

184. Desai, T. and Shea, L.D. (2017) Advances in islet encapsulation technologies. Nature Reviews Drug Discovery 16 (5), 338-350.

185. Vegas, A.J. et al. (2016) Combinatorial hydrogel library enables identification of materials that mitigate the foreign body response in primates. Nature biotechnology 34 (3), 345.

186. Carlsson, P.-O. et al. (1998) Measurements of oxygen tension in native and transplanted rat pancreatic islets. Diabetes 47 (7), 1027-1032.

187. Carlsson, P.-O. et al. (2001) Markedly decreased oxygen tension in transplanted rat pancreatic islets irrespective of the implantation site. Diabetes 50 (3), 489-495.

188. Dionne, K.E. et al. (1993) Effect of hypoxia on insulin secretion by isolated rat and canine islets of Langerhans. Diabetes 42 (1), 12-21.

189. Ziello, J.E. et al. (2007) Hypoxia-Inducible Factor (HIF)-1 regulatory pathway and its potential for therapeutic intervention in malignancy and ischemia. Yale J Biol Med 80 (2), 5160 .

190. Barkai, U. et al. (2013) Enhanced oxygen supply improves islet viability in a new bioartificial pancreas. Cell transplantation 22 (8), 1463-1476.

191. Jung, H.S. et al. (2013) Hypoxic resistance of hypodermically transplanted pancreatic islets by using cell-absorbable antioxidant Tat-metallothionein. Journal of Controlled Release 172 (3), 1092-1101.

192. McQuilling, J.P. and Opara, E.C. (2017) Methods for Incorporating Oxygen-Generating Biomaterials into Cell Culture and Microcapsule Systems. Cell Microencapsulation: Methods and Protocols, 135-141.

193. Pedraza, E. et al. (2012) Preventing hypoxia-induced cell death in beta cells and islets via hydrolytically activated, oxygen-generating biomaterials. Proceedings of the National Academy of Sciences 109 (11), 4245-4250.

194. Chae, S.Y. et al. (2002) Effect of cross-linked hemoglobin on functionality and viability of microencapsulated pancreatic islets. Tissue engineering 8 (3), 379-394. 
195. Jansson, L. and Hellerström, C. (1983) Stimulation by glucose of the blood flow to the pancreatic islets of the rat. Diabetologia 25 (1), 45-50.

196. Carlsson, P.-O. et al. (1996) Pancreatic islet blood flow in normal and obese-hyperglycemic (ob/ob) mice. American Journal of Physiology-Endocrinology And Metabolism 271 (6), E990E995.

197. Lifson, N. et al. (1989) Blood Flow to the Rabbit Pancreas with Special Reference to the Islets of Gngerhans. Gastroenterology 79, 408-473.

198. Brissova, M. et al. (2006) Pancreatic islet production of vascular endothelial growth factor$a$ is essential for islet vascularization, revascularization, and function. Diabetes 55 (11), 29742985.

199. Johansson, M. et al. (2008) Prolactin treatment improves engraftment and function of transplanted pancreatic islets. Endocrinology 150 (4), 1646-1653.

200. Yin, N. et al. (2016) VEGF-conjugated alginate hydrogel prompt angiogenesis and improve pancreatic islet engraftment and function in type 1 diabetes. Materials Science and Engineering: C 59, 958-964.

201. Miao, G. et al. (2006) Dynamic Production of Hypoxia-Inducible Factor-1a in Early Transplanted Islets. American journal of transplantation 6 (11), 2636-2643.

202. Linn, T. et al. (2003) Angiogenic capacity of endothelial cells in islets of Langerhans. The FASEB journal 17 (8), 881-883.

203. Vlahos, A.E. et al. (2017) Modular tissue engineering for the vascularization of subcutaneously transplanted pancreatic islets. Proceedings of the National Academy of Sciences 114 (35), 9337-9342.

204. Ryan, E.A. et al. (2002) Successful islet transplantation. Diabetes 51 (7), 2148-2157.

205. Berman, D.M. et al. (2007) Interference with tissue factor prolongs intrahepatic islet allograft survival in a nonhuman primate marginal mass model. Transplantation 84 (3), 308315.

206. Barshes, N.R. et al. (2005) Inflammation-mediated dysfunction and apoptosis in pancreatic islet transplantation: implications for intrahepatic grafts. Journal of leukocyte biology 77 (5), 587-597.

207. Stagner, J. et al., Reconstructing the pancreas: restoration of normoglycemia, exocrine function, and islet innervation by islet transplantation to the pancreas, Transplantation proceedings, Elsevier, 2008, pp. 452-454.

208. Ellis, H. (2013) Anatomy of the pancreas and the spleen. Surgery (Oxford) 31 (6), 263266.

209. Alderson, D. et al. (1984) Islet cell transplantation in diabetic dogs: studies of graft function and storage. British journal of surgery 71 (10), 756-760.

210. Kaufman, D.B. et al. (1990) PURIFIED CANINE ISLET AUTOGRAFTS: FUNCTIONAL OUTCOME AS INFLUENCED BY ISLET NUMBER AND IMPLANTATION SITE. Transplantation 50 (3), 385-390.

211. Largiader, F. et al. (1979) Successful allotransplantation of an island of Langerhans. Schweizerische Medizinische Wochenschrift 109 (45), 1733-1736. 
212. Xiaohui, T. et al., Small intestinal submucosa improves islet survival and function in vitro culture, Transplantation proceedings, Elsevier, 2006, pp. 1552-1558.

213. Caiazzo, R. et al., Evaluation of alternative sites for islet transplantation in the minipig: interest and limits of the gastric submucosa, Transplantation proceedings, Elsevier, 2007, pp. 2620-2623.

214. Tchervenivanov, N. et al. (2002) Morphological and functional studies on submucosal islet transplants in normal and diabetic hamsters. Cell transplantation 11 (6), 529-537.

215. Wszola, M. et al. (2008) TransEndoscopic Gastric SubMucosa Islet Transplantation (eGSM-ITX) in pigs with streptozotocine induced diabetes-technical aspects of the procedurepreliminary report. Annals of transplantation 14 (2), 45-50.

216. Vaubel, E. (1983) The Greater Omentum: Anatomy, Physiology, Pathology, Surgery, with an Historical Survey, Springer Verlag.

217. Outzen, H.C. and Leiter, E.H. (1981) TRANSPLANTATION OF PANCREATIC ISLETS INTO CLEARED MAMMARY FAT PADS. A NEW MODEL. Transplantation 32 (2), 101-105.

218. Kim, H.-I. et al. (2010) Comparison of four pancreatic islet implantation sites. Journal of Korean medical science 25 (2), 203-210.

219. Ao, Z. et al. (1993) Survival and function of purified islets in the omental pouch site of outbred dogs. Transplantation 56 (3), 524-529.

220. Al-Abdullah, I.H. et al. (1995) Site for unpurified islet transplantation is an important parameter for determination of the outcome of graft survival and function. Cell transplantation 4 (3), 297-305.

221. Balamurugan, A. et al. (2003) Bioartificial pancreas transplantation at prevascularized intermuscular space: effect of angiogenesis induction on islet survival. Pancreas 26 (3), 279285.

222. Pepper, A.R. et al. (2015) A prevascularized subcutaneous device-less site for islet and cellular transplantation. Nature biotechnology 33 (5), 518-523.

223. Salazar-Bañuelos, A. et al. (2008) The bone marrow as a potential receptor site for pancreatic islet grafts. Archives of medical research 39 (1), 139-141.

224. Cantarelli, E. et al. (2009) Bone marrow as an alternative site for islet transplantation. Blood 114 (20), 4566-4574. 



\title{
Chapter 3
}

Degradation kinetics and changes of poly(ester-urethane) and poly(ethylene glycol terephtalatepolybutylene terephthalate) thin films used for internal biomedical applications.

\author{
E. Hadavi \\ A.A. van Apeldoorn \\ H.B.J. Karperien
}




\section{Abstract}

In this study we have compared the structure, properties and degradation behavior of two distinct polymers, a poly(ester urethane) and block copolymer consisting of polyethyleneglycol-terephthalate polybutylene-terephthalate (PEOT-PBT), that are used as biomaterials for the fabrication of scaffolds in cell delivery devices. The polyester urethane in this study is a degradable segmented copolymer composed of oligoester segments, based on the random copolymerization of lactide and $\mathcal{\varepsilon}$ caprolactone, and short segments containing carbamate groups, and is produced by Polyganics BV in the Netherlands. The PEOT-PBT block copolymer used in this study has quite distinct properties compared to the polyurethane based polymer and contains single PEOT segments connected to PBT segments with a degree of polymerization of 30. The molecular weight of the polyetheylene glycol used in the synthesis is 4000, while the mass ratio percentage of PEOT to PBT is 30:70\% (400OPEOT3OPBT70). Stress-strain and DSC experiments revealed that PEOT-PBT is a semi-crystalline material with high modulus (260 MPa) and melting point of $218{ }^{\circ} \mathrm{C}$, while the poly(ester urethane) is an amorphous material with an E-modulus of $32 \mathrm{MPa}$. Moreover, the maximum stress of the PEOT-PBT (11,1 MPa) was higher than that of the poly(ester urethane) $(7,2 \mathrm{MPa})$, whereas the elongation at break of both materials was 225\%. A degradation study on both polymers was performed at pH 5, 7.4 and 9 using phosphate buffered saline (PBS) at $37^{\circ} \mathrm{C}$ for 34 weeks. At intermittent time points, polymer weight loss was measured. ${ }^{1} \mathrm{H} N M R,{ }^{13} \mathrm{C} N M R$, FTIR spectroscopy, differential scanning calorimetry (DSC) and scanning electron microscopy were used to analyze the remaining material. Results indicated that degradation of the poly(ester urethane) and PEOT-PBT proceded through hydrolysis of ester groups resulting in polymer segments containing hydroxyl and carboxylic acid functional groups. The PEOT-PBT polymer displayed minimal mass loss over time, while the poly(ester urethane) had lost $60 \%$ of its initial mass in 34 weeks. In addition, the poly(ester urethane) became brittle after 5 weeks, and disintegrated by complete fragmentation of the samples after 34 weeks. In contrast, the PEOT-PBT retained its shape during 34 weeks while the sample surface showed increased roughness and irregularities. The results show that the poly(ester urethane) is a less suitable material to be applied in 
long term tissue supporting, or cell delivery, devices while PEOT-PBT is more suitable to produce longterm form stable three dimensional tissue engineered scaffolds.

\section{Introduction}

During the past fifty years research on biodegradable materials has led to a wide range of polymeric materials that can be used in various biomedical applications like drug delivery systems, implantable medical devices and scaffolds for tissue engineering. Since their development and application as flexible and elastic materials used in surgical sutures, biodegradable aliphatic polyesters composed of e.g. glycolyl, lactyl and caprolactoyl moieties have attracted most interest [1-3]. These types of polyesters are biocompatible and their degradation products generated by hydrolysis do not evoke cytotoxic effects $[4,5]$. Based on these early successes several other biodegradable polymeric materials were developed for both biomedical, and environmental, applications such as; poly(ester urethane)s [6], poly(ester amide)s [7], polydepsipeptides [8], poly(anhydride)s [9], poly(ortho ester)s [10] and poly(ester)s based on terephthalates (PEOT-PBT) [11].

Polymers composed of the aforementioned specific monomer units allow control of material properties to comply with the intended clinical application, and further optimization to match and support tissue repair at the location of interest. Living ring-opening polymerization of lactones, like glycolide, lactide and $\varepsilon$-caprolactone, have provided much insight in the mechanistic aspects of the synthesis reaction and control over the resulting molecular structure of these polymers. Nowadays, poly(esters) can be prepared in a reproducible accurate manner using a wide variety of catalysts leading to materials with a precise molecular weight distribution and specific molecular weight. Nevertheless, restrictions in sequential polymerization of the above mentioned monomers limit possible outcomes. For example in block copolymerization, $\varepsilon$-caprolactone can only be polymerized first 
and then followed by lactide, but not vice versa [12]. Additionally, catalyst and reaction conditions can greatly affect transesterification and racemization reactions which can lead to undesired polymer microstructures.

A commonly used catalyst in the ring opening polymerization of lactones to aliphatic polyesters is stannous octoate, which combined with an alcohol as an initiator can be applied to reliably synthesize polymers in bulk at elevated temperatures [13]. Although the final polymer composition may reflect the feed ratio of the monomers, the differences in monomer reactivity can lead to tapered polymer chains. All these aforementioned effects combined will ultimately have significant influence on the thermal and mechanical properties, and hydrolytic degradation of the final product. Precise control over the polymerization temperature, reaction time and purity of the lactones used as building blocks therefore is needed to ensure that the desired molecular structure is produced in an efficient manner [13].

Poly(ester urethane)s are generally prepared by an addition reaction of oligoesters, containing hydroxyl functional end-groups and diisocyanates, or isocyanate end-functionalized oligoesters and low molecular weight diols $[14,15]$. Poly(ester urethane)s have been investigated as biomaterials used as meniscal inserts [16], endovascular prosthesis and vascular tissue engineering [17], and as a gastro-intestinal anastomosis protector [6, 18], and are clinically used as Nasopore-NF® produced by Polyganics BV, the Netherlands. In general these materials are amorphous [19], elastic [5, 20] and their degradation time can range from a few weeks to a few months depending on the $\mathrm{pH}[21]$.

PEOT-PBT polymers having different molecular compositions have been extensively investigated as cement restrictor in revision hip implant surgery, drugdelivery systems and tissue engineered chondrocyte delivery devices in a cartilage repair clinical trial [22-24]. The main advantage of these block-copolymers is that, depending on the clinical application the polymer composition, by varying the soft (PEOT) to hard (PBT) block ratio, and the chain length of polyethylene glycol used 
during synthesis, the material properties can be tuned to cover a wide spectrum of physical and mechanical and degradation characteristics.

Here we report on the relationship between the polymer microstructural changes of a PEOT-PBT and poly(ester urethane) and their physical and mechanical properties during a controlled degradation study to predict in vivo behavior. The changes in material properties were compared after a controlled in vitro degradation study at $\mathrm{pH} 5,7.4$ and 9 at $37^{\circ} \mathrm{C}$ over a period of 34 weeks. Microstructural and chemical analysis of these polymeric materials was performed using ${ }^{1} \mathrm{H}$ NMR and ${ }^{13} \mathrm{C}$ NMR spectroscopic techniques, and weight loss. The analysis was subsequently related to the polymer physical and mechanical properties and structural changes.

\section{Materials and Methods}

\section{Materials}

Similar sized thin films of a PEOT-PBT and poly(ester urethane) were made by solvent casting. PEOT-PBT was produced by Polyvation BV (Groningen, the Netherlands, under the trade name PolyActive ${ }^{\mathrm{TM}}$ ). It is composed of poly (ethylene oxide) with a molecular weight ( $\mathrm{Mw}$ ) of 4000 , and the weight percentage $(\mathrm{wt} \%)$ of PEOT and PBT blocks was $30 \mathrm{wt} \%$ and $70 \mathrm{wt} \%$, respectively (4000PEOT30PBT70) [25]. The poly(ester urethane) was provided by Polyganics BV (Groningen, the Netherlands). For preparation of polymer solutions chloroform (Merck, Darmstadt, Germany) and 1,1,1,3,3,3-hexafluoro-2-isopropanol (Biosolve, Valkenswaard, the Netherlands) were used as solvents $[5,6]$.

\section{Polymer film fabrication}

Thin films with a thickness of $40 \mu \mathrm{m}$ were fabricated using solvent casting. The PEOT-PBT was dissolved in a 65:35 (w/w) mixture of chloroform and 1,1,1,3,3,3- 
hexafluoro-2-isopropanol at a concentration of $15 \mathrm{wt} \%$ and casted on silicon wafers at room temperature. The polymer films were dried under a continuous nitrogen stream for $4 \mathrm{~h}$ followed by immersion in ethanol to remove residual solvent and easily peeling from the substrate. Subsequently, the polymer films were dried in a vacuum oven (Heraeus, Hanau, Germany) at $30^{\circ} \mathrm{C}$ for 3 days. Poly(ester urethane) films with a thickness of $40 \mu \mathrm{m}$ were fabricated in a similar way from a $5 \mathrm{wt} \%$ polymer solution in chloroform.

\section{Spectroscopy}

Each polymer was analyzed using Fourier Transformed Infrared (FTIR) spectroscopy (Spectrum 2000, Perkin- Elmer, UK) in the $400-4000 \mathrm{~cm}^{-1}$ wavelength region. The polymer composition and chain microstructure were determined using NMR spectroscopy (Bruker Avance spectrometer operating at $400 \mathrm{MHz}\left({ }^{1} \mathrm{H} \mathrm{NMR}\right)$ and $\left.110.6 \mathrm{MHz}\left({ }^{13} \mathrm{C} \mathrm{NMR}\right)\right)$. The PEOT-PBT and poly(ester urethane) were dissolved in $\mathrm{CDCl}_{3}$.

\section{Degradation}

Controlled in vitro hydrolytic degradation was carried out by immersing $20 \mathrm{mg}$ polymer films in phosphate-buffered saline solution (PBS) at $\mathrm{pH} 5, \mathrm{pH} 7.4$ or $\mathrm{pH}$ 9 at $37^{\circ} \mathrm{C}$. The samples were incubated on a table top orbital shaker at $150 \mathrm{rpm}$. At predetermined time points samples $(n=3)$ were removed, rinsed in Mili-Q water and air dried. Subsequently, samples were weighed and FTIR spectra, followed by 1H NMR spectra of dissolved samples in DMSO or CDCl3 were taken. The changes in surface morphology of the degraded polymer films were examined at subsequent time points using scanning electron microscopy (JEOL JSM-6400 SEM, Japan). Before scanning electron microscopy samples were gold coated using a Cressington 108 auto sputter coater (Cressington, Watford UK). 


\section{Mechanical properties}

The mechanical properties of dry polymer films were determined using a universal tensile testing device (Zwick Z020, Germany). Samples with a thickness of $40 \mu \mathrm{m}$ were cut according to ASTM specifications ( $50 \mathrm{~mm} \times 4 \mathrm{~mm}$ ). Tensile tests were carried out at room temperature using a $0.01 \mathrm{~N}$ pre-load, a crosshead speed of $50 \mathrm{~mm} / \mathrm{min}$ and an initial grip to grip separation of $30 \mathrm{~mm}$.

\section{Contact angles}

Water contact angles were measured by sessile contact angle waterdrop measurements $(\sim 10 \mu \mathrm{L})$ using an electronically regulated Hamilton syringe containing a curved needle. Contact angles were determined using SCA20 software (DataPhysics Instruments GmbH, Filderstadt, Germany).

\section{Results and Discussion}

The PEOT-PBT was synthesized by the polycondensation of dimethyl terephthalate, 1,4-butanediol and a poly(ethylene oxide) (PEO) MW $4000 \mathrm{~g} / \mathrm{mol}$ (Scheme 1A)[25]. The poly(ester-urethane) requires a multistep preparation and was synthesized by reaction of an a, $\omega$-isocyanate end functionalized pre-polyester and an a, $\omega$-hydroxy bis-carbamate (Scheme 1b). The pre-polyester is prepared by ring-opening polymerization of DL-lactide and $\varepsilon$-caprolactone using 1,4-butanediol as an initiator. The hydroxyl end groups are subsequently reacted with an excess of 1,4-butanediisocyanate providing isocyanate end-groups. The hydroxyl functional biscarbamate chain extender was prepared by reaction of 1,4-butanediisocyanate 
in an excess of 1,4-butanediol. Condensation of the two compounds ultimately results in the poly(ester urethane) [26].

\section{A PolyActive}
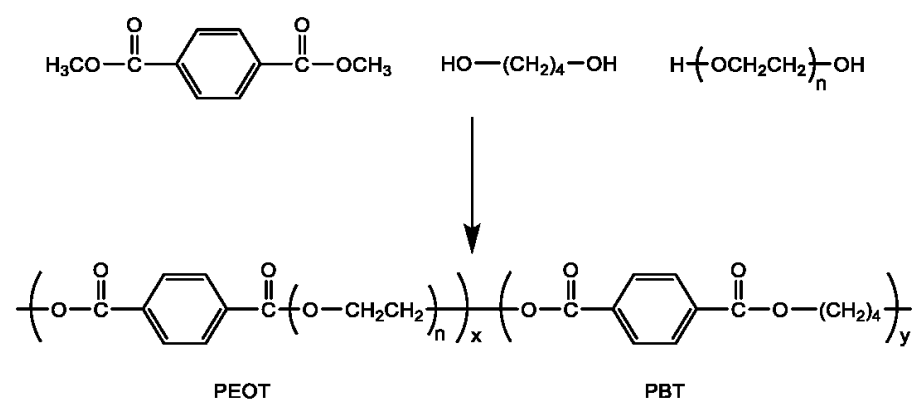

\section{B Poly(ester urethane)}

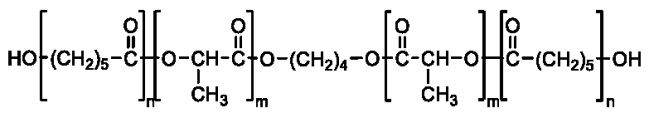

$$
\begin{aligned}
& \downarrow \mathrm{O}=\mathrm{C}=\mathrm{N}-\left(\mathrm{CH}_{2}\right)_{4}-\mathrm{N}=\mathrm{C}=\mathrm{O}
\end{aligned}
$$

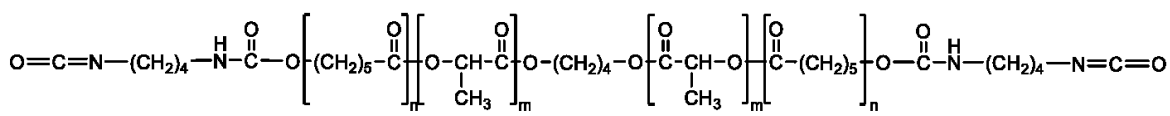

$$
\begin{aligned}
& \mathrm{HO}-\left(\mathrm{CH}_{2}\right)_{4}-\mathrm{O}-\mathrm{O} \mathrm{C}-\mathrm{H}-\left(\mathrm{CH}_{2}\right)_{4}-\mathrm{N}-\stackrel{\mathrm{O}}{\mathrm{C}}-\mathrm{O}-\left(\mathrm{CH}_{2}\right)_{4}-\mathrm{OH}
\end{aligned}
$$

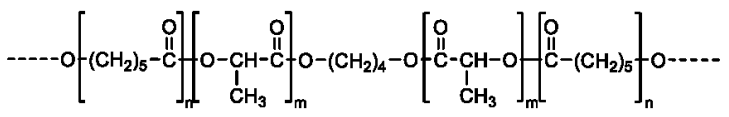

$$
\begin{aligned}
& \square
\end{aligned}
$$

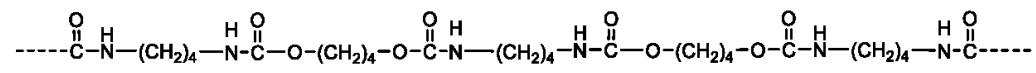


Scheme 1. A) General scheme of the synthesis and structure of PEOT-PBT. B) Synthesis and structure of the segmented poly(ester-urethane) prepared from i) a prepolyester by random copolymerization of lactide and $\varepsilon$-caprolactone and end group modified with a diisocyanate and ii) chain extension was done with a a,w-diol prepared from 1,4diisocyanatobutane and 1,4-butanediol.

The ${ }^{1} \mathrm{H}-\mathrm{NMR}$ spectrum of the PEOT-PBT is presented in Figure 1, left. From the ratio of the signals at 3.4 (PEO), 4.4 (butyl ester) and 8.1 (aromatic) ppm the molar ratio and subsequently weight of the PEOT and PBT segments was calculated. The microstructure of PEOT-PBT polymer chains can be described as PEOT segments connected to butyl terephthalate (PBT) segments with a degree of polymerization of at least 30 .

The ${ }^{1} \mathrm{H}$-NMR spectrum of the poly(ester urethane) (Figure 1, right) reveals that the integral ratio between the signal of the D,L-lactide methine protons at 4.85$5.25 \mathrm{ppm}$ and the caprolactone $\mathrm{CH}_{2} \mathrm{C}(\mathrm{O})$ protons at $2.34 \mathrm{ppm}$ have a ratio of 1.25:1. The splitting of the signals at $\sim 2.30$ ppm shows that both CL-CL sequences $(2.25$ ppm) and DLLA-CL sequences (2.30 ppm) are present.

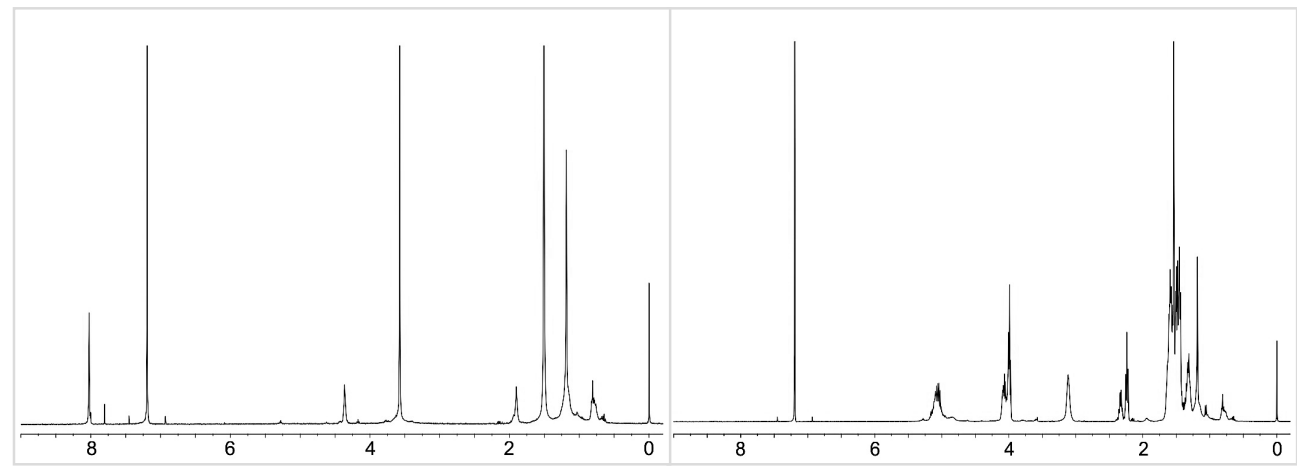

Figure 1. ${ }^{1} \mathrm{H}$ NMR spectrum of the PEOT-PBT (left) and poly(ester urethane) (right). Solvent $\mathrm{CDCl}_{3}$.

Pre-polyesters with a molecular weight of approximately 2000, applied in the synthesis of the poly(ester-urethane) are usually prepared by the ring-opening polymerization of a 50/50 (mol/mol) mixture of DL-lactide and $\varepsilon$-caprolactone using 1,4-butanediol as an initiator and stannous octoate as a catalyst at $130^{\circ} \mathrm{C}$ 
(Figure 2). According the patent and manufacturer description (Polyganics BV) the pre-polyester polymerization was performed at a monomer to 1,4-dutanediol molar ratio of $0.28 / 0.01887(\mathrm{~mol} / \mathrm{mol})$. Based on the molar ratio the calculated prepolyester chain length will contain on average 7.4 units on each side of the butane diol central unit, leading to a total molecular weight (MW) of $2076 \mathrm{~g} / \mathrm{mol}$ assuming equimolar incorporation of both monomer units.

The initiating species formed in the equilibrium between the stannous octoate and the alcohol is the OctSnOR and finally the $\mathrm{Sn}(\mathrm{OR})_{2}$ (Figure 3). This is followed by a coordination insertion reaction of the stannous alcoholate with the monomer. Considering the applied excess amount of initiator over the catalyst and high temperature, the alcohol can act as a chain transfer agent leading to transesterification reactions. As described by Baimark and Molloy [26] such reactions will increase the number of chains initiated. Although no straightforward effect of these processes on molecular weight were found, the measured molecular weight generally resembles the molar ratio of monomer to initiating groups applied in the polymerization reaction.

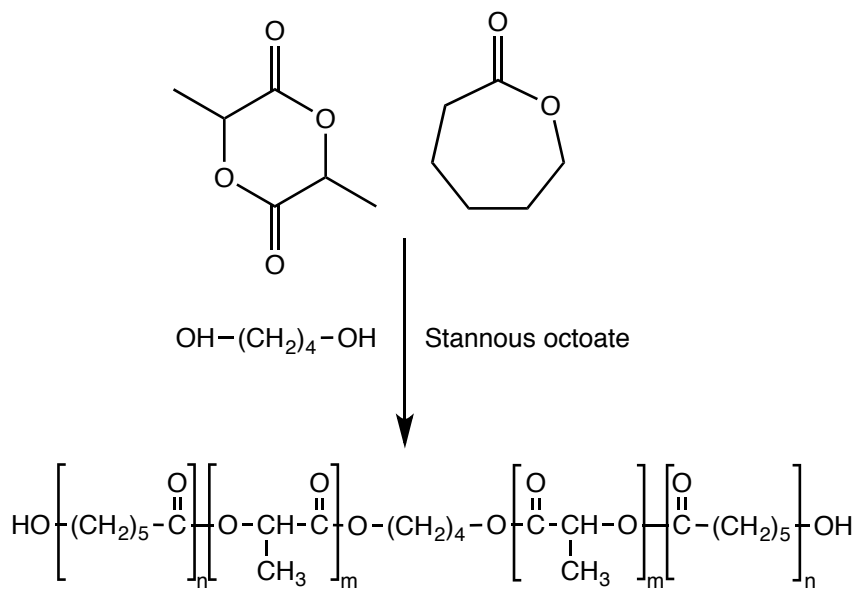


Figure 2. Structure of the pre-polyester prepared by the bulk ring opening polymerization of DL-lactide and $\varepsilon$-caprolactone using stannous octoate as a catalyst and 1,4-butanediol as an initiator.

A.



B.

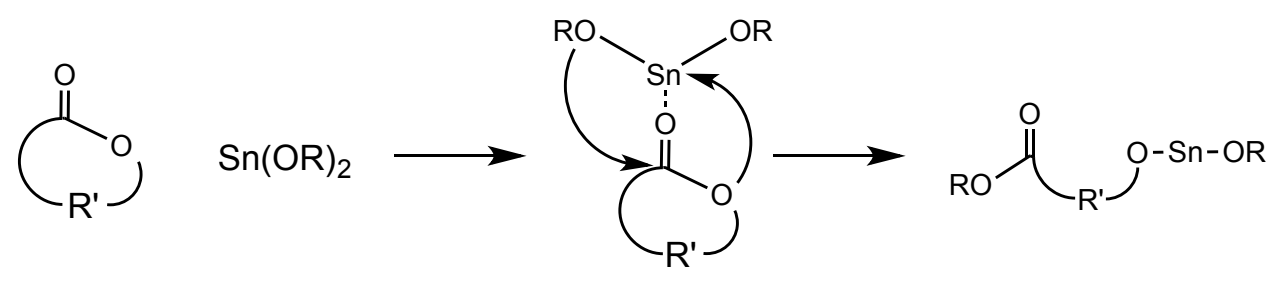

Figure 3. Stannous alkoxide formation (A), and ring opening polymerization mechanism of lactones (B).

As an example of such a pre-polyester, the long reaction time of 24 hours used in this ring-opening polymerization ensured almost complete conversion of the monomers as shown by its ${ }^{1} \mathrm{H}$ NMR spectrum (Figure 4). Only a very small amount of remaining monomer can be observed by the broad signal at $2.7 \mathrm{ppm}$, which belongs to the methylene protons of the $\varepsilon$-caprolactone next to the carboxyl group. A small amount of DL-lactide monomer was observed by the signal at $4.3 \mathrm{ppm}$. The proton signals of the 1,4-butanediol initiator overlap with the monomer signals present at 4.1 and $1.5 \mathrm{ppm}$. Addition of trifluoroacetic anhydride to the prepolyester dissolved in $\mathrm{CDCl}_{3}$ shifted the end group signal downfield to 4.3-4.5 ppm. The integral ratio of the end-group to monomer protons allowed the calculation of the molecular weight (Mn), which was $\sim 2000$, close to the theoretical Mn. 
The molar ratio of lactyl to caprolactoyl units in the pre-polyester, 1.25:1 was calculated from the integral ratio of the signal at $5.1 \mathrm{ppm}$ (lactyl units) and 2.3 ppm (caprolactoyl $\alpha$-methylene protons). The higher amount of lactyl units in the pre-polyester is the result of the well-known higher rate of ring-opening polymerization of lactide compared to $\varepsilon$-caprolactone.

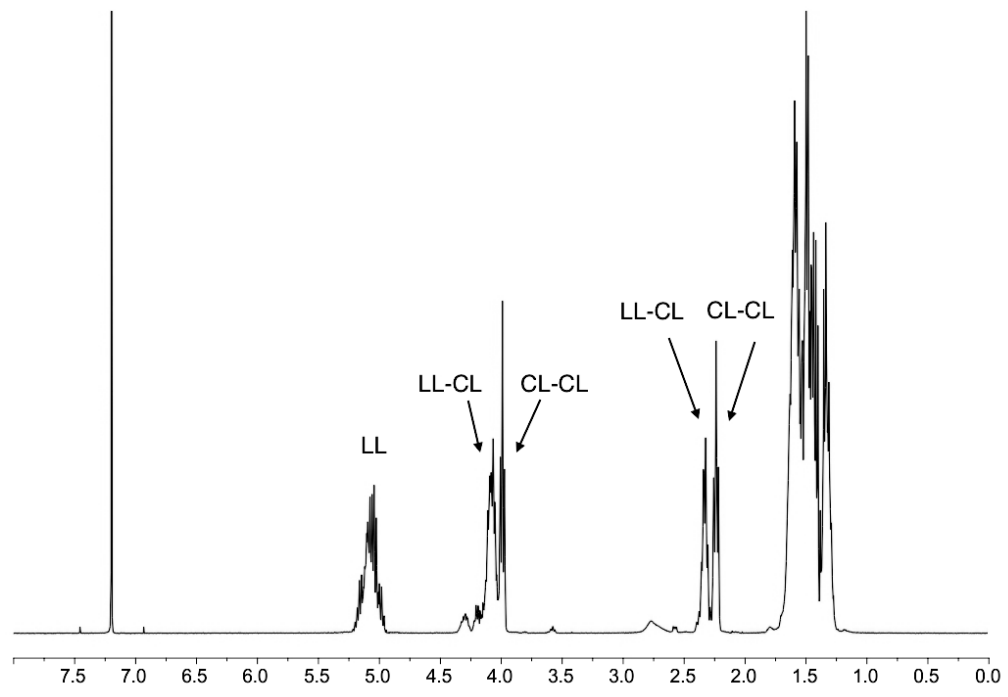

Figure 4. ${ }^{1} \mathrm{H}$ NMR spectrum of the pre-polyester with a Mn of 2000. Sequences of lactyl (L) and caprolactoyl (CL) are indicated $\left(\mathrm{CDCl}_{3}\right)$.

The molecular structure of the polyester segments in the poly(ester urethane) can be analyzed by the average sequence lengths of the monomer units, and the randomness of the polymer chains applying equations 1 to 5 . It is important to note that in the following formulae a caprolactoyl unit is abbreviated as CL and a lactide unit as LL, thus comprising two lactyl units.

The number average sequence lengths are expressed by;

$$
l_{L L}=\frac{2(L L)}{(L L-C L)}
$$




$$
l_{C L}=\frac{2(C L)}{(L L-C L)}
$$

in which (LL) and (CL) are the two monomer molar fractions and (LL-CL) is the average dyad relative molar fraction.

The random number sequence lengths are given by;

$$
\begin{aligned}
& \left(l_{L L}\right)_{\text {random }}=\frac{1}{(C L)} \\
& \left(l_{C L}\right)_{\text {random }}=\frac{1}{(L L)}
\end{aligned}
$$

The randomness of the monomers in the polymer chains is presented by;

$$
R=\frac{(L L-C L)}{2(L L)(C L)}
$$

In case of a block copolymer $\mathrm{R}$ will be 0 and in case of a random copolymer $\mathrm{R}$ is 1.

The applied monomer molar feed ratio was 50/50, whereas from the ${ }^{1} \mathrm{H}$ NMR spectrum the LL/CL ratio was calculated $47 / 53$. The random factors are therefore $1.89\left(\left(l_{L L}\right)_{\text {random }}\right)$ and $2.13\left(\left(l_{C L}\right)_{\text {random }}\right)$, respectively. The molar fractions of the different dyads were calculated from equations 6-8, in which LL-LL and CL-CL are the molar fractions of pure lactide-lactide sequences and caprolactoyl-caprolactoyl sequences, respectively, and LL-CL is the average lactyl-caprolactoyl dyad relative 
molar fraction. These values were calculated from the integral ratios of CL-CL sequences and CL-LL sequences of the signals in the ${ }^{1} \mathrm{H}$ NMR spectrum.

$$
\begin{aligned}
& (L L-C L)=1-(L L-L L)-(C L-C L) \\
& (L L-L L)=0.47-\frac{I_{2.30}}{I_{2.2-2.4}+I_{5.2}} \\
& (C L-C L)=0.53 * \frac{I_{2.2}}{I_{2.2-2.4}}
\end{aligned}
$$

The values calculated for (LL-LL), (CL-CL) and (LL-CL) were 0.20, 0.27 and 0.53, respectively. The random factor $\mathrm{R}$ has a value of 1.06, which reveals an almost random character of the polymer chains. The number average sequence lengths are 1.8 for the lactyl units and 2 for the caprolactoyl units according equations 1 and 2.

The ${ }^{1} \mathrm{H}$ NMR spectrum provides no information on the presence of caprolactoyllactyl-caprolactoyl (CL-L-CL) sequences due to possible transesterification reactions and therefore only give an indication on the molecular structure of the pre-polyester chains.

The sequence of monomer units in the polyester chains can also be obtained from the ${ }^{13} \mathrm{C}$ NMR spectrum according to an analysis by Vanhoorne et al. [27]. This method provides additional information for the carbonyl region of the spectrum, to which triad sequences of lactyl and caprolactoyl units can be assigned. As depicted in Figure 5 the different sequences of CL to L units in the carbonyl region are presented. It has to be noted that a representative sample of the prepolyester was not available. The average monomer block lengths $\mathrm{L}_{\mathrm{LL}}$ and $\mathrm{L}_{\mathrm{C}}$ of the polyester segments was calculated according equations 9 and 10. 


$$
\begin{aligned}
& L_{L L}=\frac{1}{2}\left[\frac{l_{L L L}+\frac{l_{L L C}+l_{C L L}}{2}}{\frac{\left(l_{L L C}+l_{C L L}\right)}{2}+l_{C L C}}\right]+1 \\
& L_{C}=\frac{l_{C C C}+l_{L C C}}{l_{C C L}+l_{L C L}}+1
\end{aligned}
$$
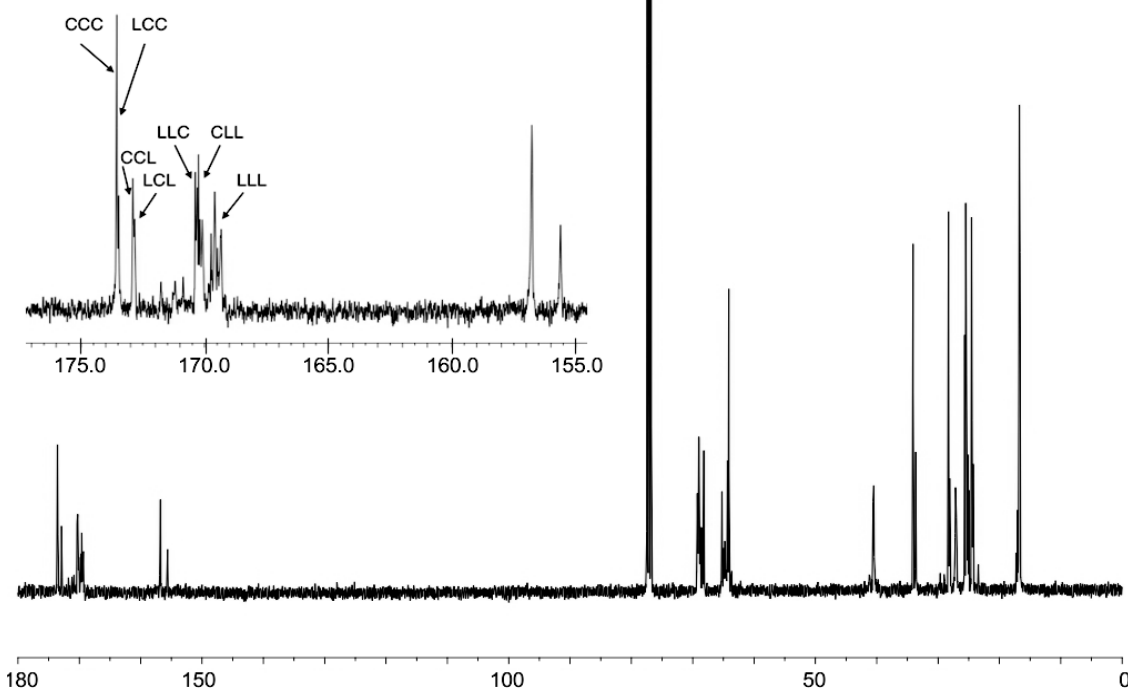

Figure 5. ${ }^{13} \mathrm{C}$ NMR spectrum of the poly(ester urethane) with in the insert the assignment of lactyl (L) caprolactoyl (C) sequences in the carbonyl region.

The values calculated from the peak intensities of $\mathrm{L}_{\mathrm{LL}}$ and $\mathrm{L}_{\mathrm{C}}$ were both 2 . These values reveal a largely randomized molecular structure. The small signal at 170.9, characteristic of CLC sequences, reveals that some inter-chain transesterification reactions have been taken place.

The pre-polyester was end-group modified with 1,4-butanediisocyanate in excess. A large excess of the diisocyanate was applied to decrease the chances of oligomerization reactions from occurring, which cannot be fully excluded. 


\section{Degradation}

In vitro degradation of the poly(ester urethane) and PEOT-PBT was followed by incubation of equally sized polymer films $(\sim 20 \mathrm{mg})$ in PBS buffer with a $\mathrm{pH}$ of 5 , 7.4 or 9 to mimic possible changes in material properties which could occur in vivo in a controlled manner over time. At regular time intervals polymer samples were removed from the incubation buffer and rinsed in water and air dried. Sample weight and thermal properties were determined, FTIR spectra and ${ }^{1} \mathrm{H}$ NMR spectra were recorded, and structural changes were assessed by scanning electron microscopy of each sample.

Macroscopically the PEOT-PBT films appeared unchanged during the entire incubation period of 34 weeks, whereas the poly(ester urethane) films completely disintegrated into small fragments after 7 weeks. Electron micrographs of poly(ester urethane) films revealed that after 5 weeks cracks start to appear at the surface. After 34 weeks, the films showed complete fragmentation (Figure 6). In contrast, PEOT-PBT films can still be handled up to 34 weeks without any macroscopically visible signs of deterioration. High magnification electron micrographs revealed some surface irregularities and that some cracks start to show at 34 weeks incubation. These findings indicate that extensive bulk degradation of the poly(ester urethane) takes place starting before 7 weeks, in stark contrast to PEOT-PBT, which only shows signs of some surface erosion after 34 weeks $[28,29]$.
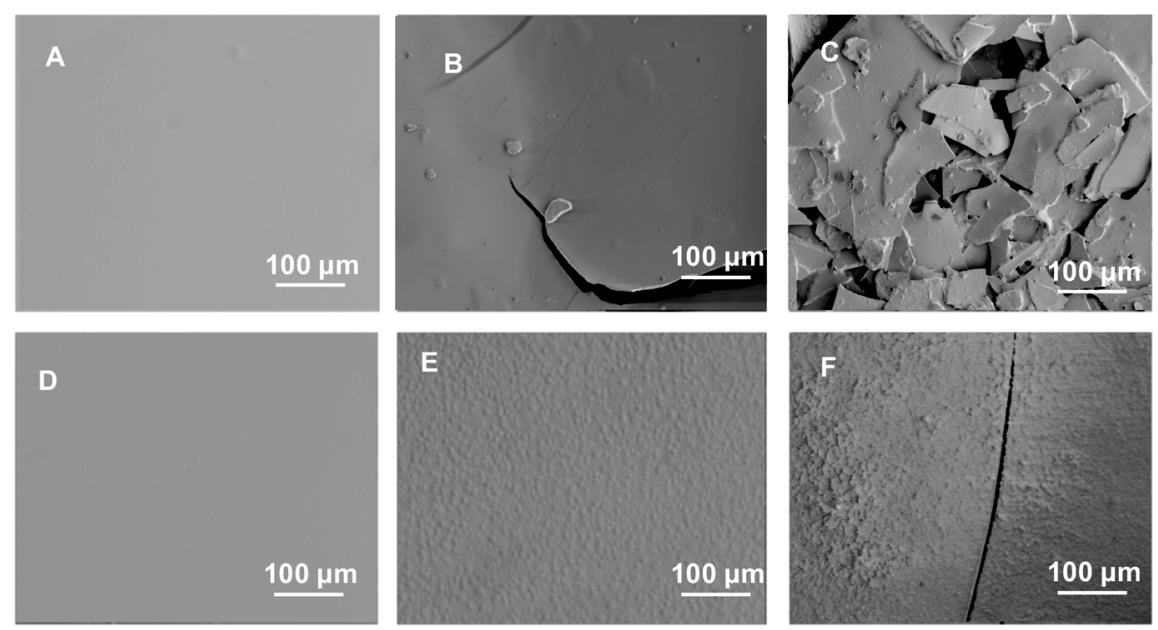
Figure 6. SEM images of the surface of the poly(ester urethane) and PEOT-PBT polymer films at different time points of degradation. A) poly(ester urethane) film at day 1 ; B) poly(ester urethane) film after 5 weeks in $\mathrm{PBS}$ at $\mathrm{pH}$ 9; C) poly(ester urethane) film after 34 weeks in PBS at pH 9; D) PEOT-PBT film at day1; E) PEOT-PBT film after 12 weeks in PBS at $\mathrm{pH}$ 9; F) PEOT-PBT film after 34 weeks in PBS at pH 9.

Degradation of the PEOT-PBT and poly(ester urethane) polymers proceeds in both materials through hydrolysis of the ester groups, leading to polymer segments with hydroxyl and carboxylic acid functional groups [30-32]. The carboxylic acid groups may accelerate the hydrolysis of the polyester segments in an autocatalytic process $[33,34]$. The degradation rate of PEOT-PBT is very slow and was highest at $\mathrm{pH}$, a value which is not physiologically relevant. After 34 weeks of incubation, the films retained their shape and showed minor weight loss at a $\mathrm{pH}$ of 7.4. Degradation of the poly(ester urethane) appeared much faster and was independent of $\mathrm{pH}$. After 34 weeks only fragments of these polymer films were observed. Besides hydrolysis as the main driving force for chain scission and degradation of these materials, it cannot be entirely excluded that also oxidation reactions can contribute to the overall degradation processes of both polymers [35].
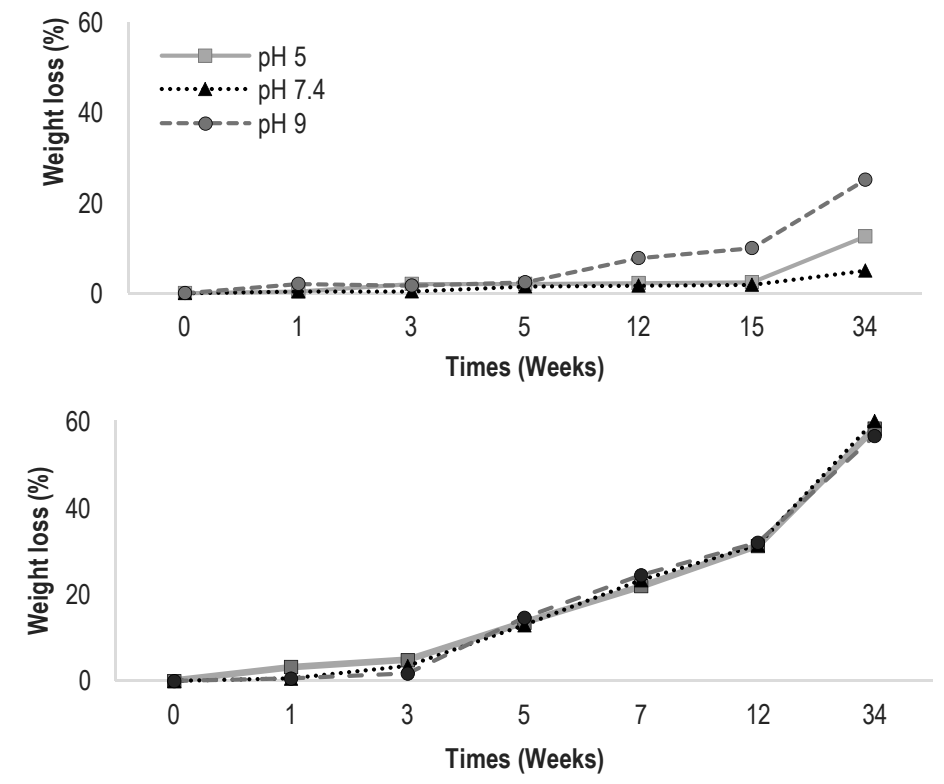

Figure 7. Weight loss of the PEOT-PBT (upper panel) and poly(ester urethane) polymer films (lower panel) incubated in PBS at $\mathrm{pH} 5,7.4$ or 9 in time. 
Differential scanning calorimetry (DSC) revealed that PEOT-PBT is a semicrystalline material with a glass transition temperature of approximately $-45^{\circ} \mathrm{C}$ and a melting transition temperature at $218^{\circ} \mathrm{C}$. No changes in these values were observed after prolonged incubation in PBS at different $\mathrm{pH}$ values during 34 weeks. The thermal properties of the amorphous poly(ester urethane) revealed a glass transition temperature at $-6.1^{\circ} \mathrm{C}$ with a $\Delta \mathrm{Cp}$ value of $0.47 \mathrm{~J} \cdot \mathrm{g}^{-1} .{ }^{\circ} \mathrm{C}^{-1}$. At $105^{\circ} \mathrm{C}$ a very small transition was observed, which can likely be explained by the organization in the polymer due to hydrogen bonding of the carbamate linkages. The $\mathrm{Tg}$ fully disappeared showing that polyester units had hydrolyzed into smaller segments.

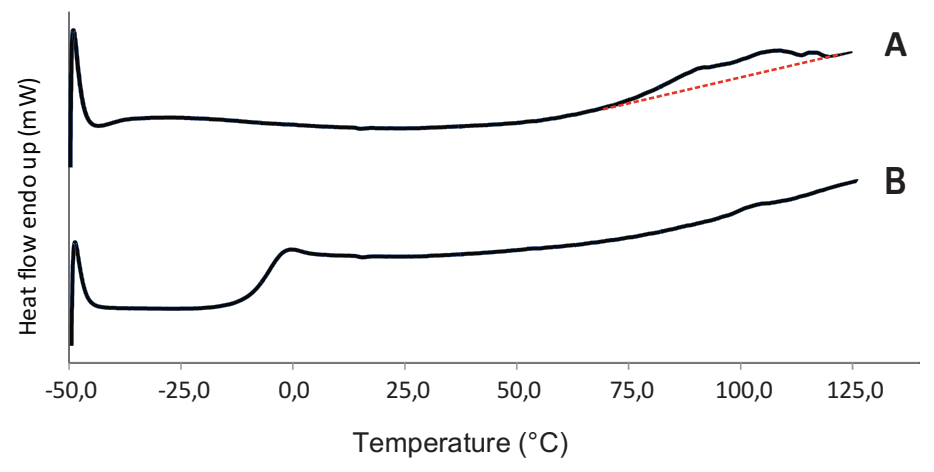

Figure 8. DSC thermograms of the poly(ester urethane) (A) before and (B) after degradation during 7 weeks in $\mathrm{PBS}, \mathrm{pH} 9$ and at $37^{\circ} \mathrm{C}$.

Upon degradation by hydrolysis the transition at $\sim 100^{\circ} \mathrm{C}$ becomes more defined but it still is very broad. The observed increase in crystallinity of the poly(ester urethane) may be due to the formation of tetra-carbamate hydrogen bonded segments with short oligoester moieties on the ends as presented in Figure 9. 


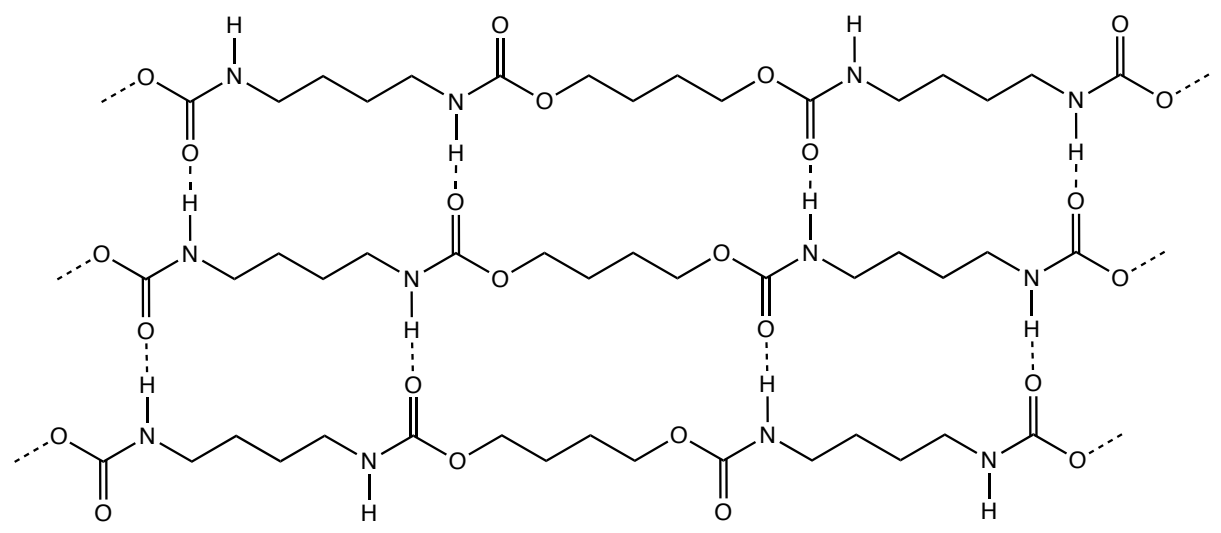

Figure 9. Representation of hydrogen bonded tetra-carbamate segments. Oligoester moieties present on both ends are not depicted.

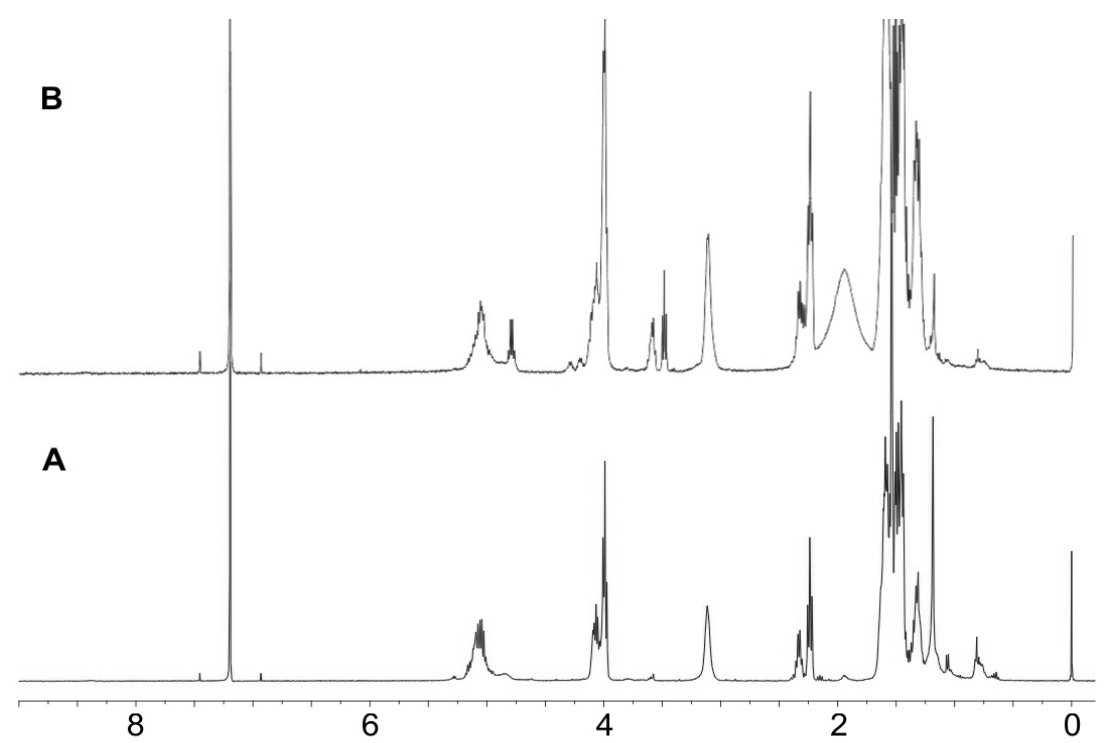

Figure 10. ${ }^{1} \mathrm{H}$ NMR spectrum of the non-degraded poly(ester urethane) (A) and after degradation for 12 weeks at $\mathrm{pH} 5$ (B) The polymer was dissolved in CDCl3.

A characteristic ${ }^{1} \mathrm{H}$ NMR spectrum of the poly(ester urethane) determined after degradation in PBS at pH 5 after 12 weeks is presented in Figure 10. The spectral data show the presence of lactyl and caprolactoyl end groups, revealing hydrolysis of ester groups has taken place. At later time points the recovered polymer fragments became difficult to dissolve in deuterated chloroform. The material 
could, however, be dissolved in deuterated DMSO, and the ${ }^{1} \mathrm{H}$ NMR data revealed a decrease in the signal of lactyl methine and caprolactoyl $\mathrm{CH}_{2}-\mathrm{C}(\mathrm{O})$ methylene protons compared to the $3.1 \mathrm{ppm} \mathrm{CH}_{2} \mathrm{NHC}(\mathrm{O})$ proton signal.

Besides NMR, the degradation of the polymer films was also monitored by FTIR spectroscopy. In poly(ester urethane) films, hydrolysis of ester bonds can clearly be observed (Figure $12 \mathrm{~A}$ ) from the ratio between the carbonyl absorption of ester bonds at $1728 \mathrm{~cm}^{-1}$ and carbamate bonds at $1680 \mathrm{~cm}^{-1}$. The appearance of a broad absorption peak at $3700 \mathrm{~cm}^{-1}$ reveals the presence of hydroxyl and carboxylic acid groups as a result of ester hydrolysis. Only minor changes were observed for the PEOT-PBT after degradation. Hydrolysis of the ester bonds between the PEO and terephthalate units have been described by others to be most susceptible to hydrolysis [36, 37]. Because of the low number of these linkages in the polymer chain and the long PBT segments only minor changes in film structure and consistency were observed. The minor changes in crystallinity and brittle characteristic of the polymer films upon degradation indicate this material degrades at a very slow rate by both surface erosion and bulk degradation.

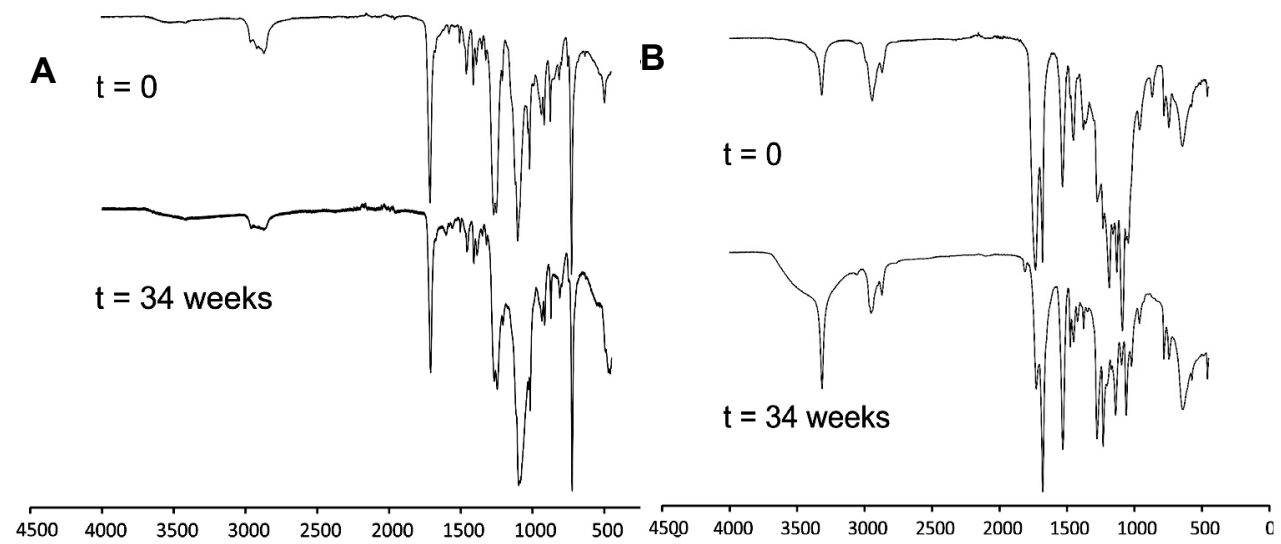

Figure 12. FTIR spectrum of A) PEOT-PBT films at day 1 and 34 weeks after degradation in $\mathrm{PBS}$ at $\mathrm{pH} 9 \mathrm{~B}$ ) poly(ester urethane) films at day 1 and 34 weeks after degradation test in $\mathrm{PBS}$ at $\mathrm{pH} 9$. 
Besides differences in degradation the mechanical properties of these two polymer are also different. The stress-strain curves of the polymers indicated that the E-modules of the poly(ester urethane) (32 MPa) was 8 times lower than that of PEOT-PBT (260 MPa) (Figure 11). Moreover, the maximum stress of PEOT-PBT (11.1 Mpa) was higher than the maximum stress of the poly(ester urethane) (7.2 Mpa). Interestingly, both polymers have an almost similar elongation at break of $225 \%$.

A previous study of Deschamps et al. [38] on the tensile behavior of PEOT-PBT polymers, 1000PEOT30PBT70, 1000PEOT55PBT45, and 1000PEOT72PBT28, with increasing ratios of the hydrophilic blocks, revealed that an increase in the weight percentage of PEOT soft segments reduces the polymer strength and stiffness, accompanied by an increase in elasticity of the material. Based on those data it was concluded that the mechanical properties of this biomaterial can be tuned to match the required mechanical properties for a specific intended medical application [38].

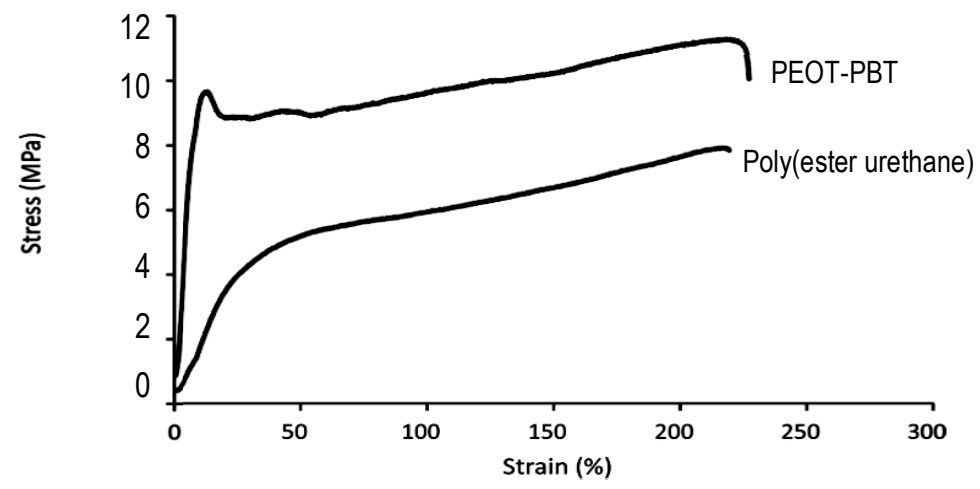

Figure 11. Stress strain curves of the PEOT-PBT and poly(ester urethane). The measurements were performed on dry specimens.

In terms of hydrophobicity, water contact angle measurements revealed considerable differences between both polymers. As expected, PEOT-PBT, containing a high PBT ratio, is a hydrophilic material $\left(39 \pm 2^{\circ}\right)$, but the poly(ester 
urethane) appears less hydrophilic $\left(75 \pm 6^{\circ}\right)$, which likely is due to the high content of carbamate groups in the polymer. The differences in hydrophobicity may not only play an important role in degradation kinetics, but also in the way proteins can adsorb to the material surface, which possibly leads to different tissue material interactions. The differences in mechanical properties show that when form stable implants or tissue engineered constructs are required, PEOT-PBT is preferred over the much softer and elastic poly(ester urethane) polymer. PEOT-PBT is clearly the most stable biomaterial in comparison to the poly(ester urethane). While the latter could serve as a short term support structure when newly formed tissue can quickly replace the implant made from this polymer, the first is more suitable as a long term implant which can provide mechanical stability and protection.

\section{Conclusions}

The physical properties of the PEOT-PBT and poly(ester urethane) segmented copolymers largely depend on the molecular structure of the polymer chains. PEOTPBT is a hydrophobic polymer with long PBT segments of semi-crystalline nature, and slowly degrades in physiological media. PEOT-PBT films retain their shape up to 34 weeks, but their surface becomes irregular and the polymer becomes increasingly brittle over time. The poly(ester urethane) has a much shorter degradation time, and polymer films lose their integrity and become brittle after 5 weeks. This poly(ester urethane) has the characteristics of a fast and bulk degradable material, showing some increase in crystallinity due to hydrogen bonding of remaining carbamate containing segments. We propose that PEOT-PBT can be used to produce stable three dimensional tissue engineered scaffolds for long-term use. The poly(ester urethane), due to its short degradation time, is less suitable for application in permanent tissue supporting devices, or long term cell delivery devices. 


\section{References}

1. Shafiq, M. et al. (2015) In situ vascular regeneration using substance P-immobilised poly

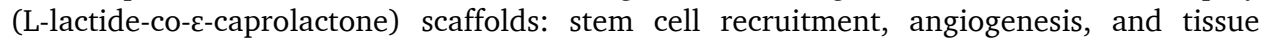
regeneration. Eur Cell Mater 30, 282-302.

2. Engelhardt, E.-M. et al. (2011) A collagen-poly (lactic acid-co-ع-caprolactone) hybrid scaffold for bladder tissue regeneration. Biomaterials 32 (16), 3969-3976.

3. Ikada, Y. and Tsuji, H. (2000) Biodegradable polyesters for medical and ecological applications. Macromolecular rapid communications 21 (3), 117-132.

4. Lim, Y.-B. et al. (2000) Biodegradable polyester, poly [a-(4-aminobutyl)-L-glycolic acid], as a non-toxic gene carrier. Pharmaceutical research 17 (7), 811-816.

5. Bruin, P. et al. (1988) Design and synthesis of biodegradable poly (ester-urethane) elastomer networks composed of non-toxic building blocks. Macromolecular Rapid Communications 9 (8), 589-594.

6. Bakker, I.S. et al. (2012) The C-seal trial: colorectal anastomosis protected by a biodegradable drain fixed to the anastomosis by a circular stapler, a multi-center randomized controlled trial. BMC surgery 12 (1), 23.

7. Rodriguez-Galan, A. et al. (2010) Degradable poly (ester amide) s for biomedical applications. Polymers 3 (1), 65-99.

8. Feng, Y. and Guo, J. (2009) Biodegradable polydepsipeptides. International journal of molecular sciences 10 (2), 589-615.

9. Griffin, J. et al. (2011) Salicylic acid-derived poly (anhydride-ester) electrospun fibers designed for regenerating the peripheral nervous system. Journal of Biomedical Materials Research Part A 97 (3), 230-242.

10. Daniels, A. et al. (1994) Evaluation of absorbable poly (ortho esters) for use in surgical implants. Journal of Applied Biomaterials 5 (1), 51-64.

11. Bartha, L. et al. (2013) A clinical feasibility study to evaluate the safety and efficacy of PEOT/PBT implants for human donor site filling during mosaicplasty. European Journal of Orthopaedic Surgery \& Traumatology 23 (1), 81-91.

12. Grijpma, D. and Pennings, A. (1991) Polymerization temperature effects on the properties of 1-lactide and ع-caprolactone copolymers. Polymer Bulletin 25 (3), 335-341.

13. Jérôme, C. and Lecomte, P. (2008) Recent advances in the synthesis of aliphatic polyesters by ring-opening polymerization. Advanced drug delivery reviews 60 (9), 10561076.

14. Bogdanov, B. et al. (1999) Thermal properties and morphology of poly (ester-urethanes) prepared from polycaprolactone-diol. Journal of thermal analysis and calorimetry 56 (3), 1115-1121.

15. Storey, R.F. et al. (1994) Hydrolyzable poly (ester-urethane) networks from L-lysine diisocyanate and D, L-lactide/€-caprolactone homo-and copolyester triols. Journal of Polymer Science Part A: Polymer Chemistry 32 (12), 2345-2363. 
16. Van Tienen, T.G. et al. (2002) Tissue ingrowth and degradation of two biodegradable porous polymers with different porosities and pore sizes. Biomaterials 23 (8), 1731-1738.

17. Nerem, R.M. and Seliktar, D. (2001) Vascular tissue engineering. Annual review of biomedical engineering 3 (1), 225-243.

18. Kolkert, J.L. et al. (2011) Protection of stapled colorectal anastomoses with a biodegradable device: the C-Seal feasibility study. The American Journal of Surgery 201 (6), 754-758.

19. Kylmä, J. and Seppälä, J.V. (1997) Synthesis and characterization of a biodegradable thermoplastic poly (ester - urethane) elastomer. Macromolecules 30 (10), 2876-2882.

20. Guan, J. et al. (2002) Synthesis, characterization, and cytocompatibility of elastomeric, biodegradable poly (ester-urethane) ureas based on poly (caprolactone) and putrescine. Journal of biomedical materials research 61 (3), 493-503.

21. Loh, X.J. et al. (2006) The in vitro hydrolysis of poly (ester urethane) s consisting of poly [(R)-3-hydroxybutyrate] and poly (ethylene glycol). Biomaterials 27 (9), 1841-1850.

22. Jansen, E.J. et al. (2009) PEOT/PBT based scaffolds with low mechanical properties improve cartilage repair tissue formation in osteochondral defects. Journal of Biomedical Materials Research Part A 89 (2), 444-452.

23. Emans, P. et al. (2013) Tissue-engineered constructs: the effect of scaffold architecture in osteochondral repair. Journal of tissue engineering and regenerative medicine 7 (9), 751756.

24. Moroni, L. et al. (2006) Polymer hollow fiber three-dimensional matrices with controllable cavity and shell thickness. Biomaterials 27 (35), 5918-5926.

25. Buitinga, M. et al. (2013) Microwell scaffolds for the extrahepatic transplantation of islets of Langerhans. PloS one 8 (5), e64772.

26. Baimark, Y. and Molloy, R. (2004) Synthesis and characterization of poly (L-lactide-co$\varepsilon$-caprolactone) copolymers: Effects of stannous octoate initiator and diethylene glycol coinitiator concentrations. Science Asia 30, 327-334.

27. Vanhoorne, P. et al. (1992) Macromolecular engineering of polylactones and polylactides. 7. Structural analysis of copolyesters of ie-caprolactone and L-or D, L-lactide initiated by triisopropoxyaluminum. Macromolecules 25 (1), 37-44.

28. Van Blitterswijk, C. et al. (1993) The effect of PEO ratio on degradation, calcification and bone bonding of PEO/PBT copolymer (PolyActive). Cells and Materials 3 (1), 2.

29. Umare, S.S. and Chandure, A.S. (2008) Synthesis, characterization and biodegradation studies of poly (ester urethane) s. Chemical Engineering Journal 142 (1), 65-77.

30. Schoonover, J.R. et al. (2001) Infrared linear dichroism study of a hydrolytically degraded poly (ester urethane). Polymer degradation and stability 74 (1), 87-96.

31. Lee, J.-W. and Gardella, J.A. (2001) In vitro hydrolytic surface degradation of poly (glycolic acid): role of the surface segregated amorphous region in the induction period of bulk erosion. Macromolecules 34 (12), 3928-3937.

32. Brown, D.W. et al. (1980) Kinetics of hydrolytic aging of polyester urethane elastomers. Macromolecules 13 (2), 248-252. 
33. Stokes, K. et al. (1995) Polyurethane elastomer biostability. Journal of biomaterials applications 9 (4), 321-354.

34. Lelah, M.D. and Cooper, S.L. (1986) Polyurethanes in medicine, CRC press.

35. Takahara, A. et al. (1991) Effect of soft segment chemistry on the biostability of segmented polyurethanes. I. In vitro oxidation. Journal of biomedical materials research 25 (3), 341-356.

36. Deschamps, A. et al. (2004) In vivo and in vitro degradation of poly (ether ester) block copolymers based on poly (ethylene glycol) and poly (butylene terephthalate). Biomaterials 25 (2), 247-258.

37. Gaharwar, A.K. et al. (2014) Amphiphilic beads as depots for sustained drug release integrated into fibrillar scaffolds. Journal of controlled release 187, 66-73.

38. Deschamps, A.A. et al. (2001) Poly (ethylene oxide)/poly (butylene terephthalate) segmented block copolymers: the effect of copolymer composition on physical properties and degradation behavior. Polymer 42 (23), 9335-9345. 



\title{
Chapter 4
}

Microcontact printing of extracellular matrix induces selfassembly of INS-1E cells in islet-like organoids with increased insulin secretion

\author{
E. Hadavi \\ J. Leijten \\ P. Jonkheijm \\ A.A. van Apeldoorn \\ H.B.J. Karperien
}




\section{Abstract}

Extracellular matrix molecules play critical roles in regulating $\beta$-cell function and viability within the pancreatic islets by providing mechanical and biological support. The isolation of islets from their native microenvironment disrupts the extracellular matrix (ECM) molecules, while interaction between the islet cells and ECM molecules such as fibronectin, collagen IV and laminin is essential for the islets function and survival. It's also thought that three-dimensional culturing of $\beta$-cells can improve cell function by providing a niche in which cells can interact with each other in a more natural manner. Conventional suspension cultures with $\beta$-cells result generally in a heterogeneous population with small and large aggregates in which cells experience different nutrient diffusion limitations that can negatively affect the physiology and functionality of these cells.

In this study, we have explored the effect of fibronectin, collagen-IV and laminin-111 on INS-1E insulinoma cells using a microcontact printing method to investigate whether a controlled environment and aggregate size would improve their endocrine function. Using this method, we produced a uniform pattern of well-defined circular spots of fibronectin, collagen-IV and laminin-111 on polydimethylsiloxane (PDMS), which resulted in autonomous assembly of cells into three-dimensional islet-like aggregates. Additionally, we indicated that the aggregates are extremely uniform in size and shape, and that the cell culture can be easily controlled leading to a highly reproducible method for three-dimensional $\beta$-cell culturing. In addition, we showed that ECM molecules can act as anchoring points for $\beta$-cells on non-adhesive materials and they can improve both function and viability of insulinoma cells. We found a significant increase in secretion of insulin by INS-1E cells cultured on microcontact printed fibronectin and collagen-IV substrates in comparison to cells that were cultured in monolayers on substrates without ECM molecules. 


\section{Introduction}

Clinical islet transplantation requires the isolation of islets from their pancreatic native environment. Isolation of islets is based on the enzymatic digestion of the pancreatic extracellular matrix (ECM), using collagenase, to separate the islets from the exocrine tissue which also affects the islet's ECM [1, 2]. ECM molecules are key microenvironmental factors that regulate numerous cellular processes in islets like, islet morphology [3], cell differentiation [4, 5], intracellular signaling [6, 7], gene expression [8, 9], cell adhesion [10], cell migration [10, 11], cell proliferation [12], insulin secretion [10, 13], and cell survival [12]. It has been reported that enzymatic disruption of the $\beta$-cell-ECM interaction is partially responsible for the rapid loss of islets post-transplantation [1]. In particular, fibronectin (FN), collagen (Col) and laminin (LN) are of high importance to $\beta$-cells function and survival [2, 14-18].

The enzymatic degradation of particular ECM molecules can lead to a loss of integrin binding, which can induce changes in phenotypic characteristics in $\beta$-cells. Consequently, this affects the islet's endocrine cell function, morphology, and survival [19]. Most notably, the interaction of integrins such as $a_{3} \beta_{1}$ and $a_{6} \beta_{1}$ with ECM was proven to affect insulin release, while $a_{v} \beta_{3}$ and $a_{v} \beta_{5}$ regulate differentiation, adhesion and migration of the $\beta$-cells $[20,21]$. Moreover, $\beta$-cells express the collagen type IV (Col4) binding $a_{v} \beta_{1}$ integrin, which is associated with insulin release, cell motility, and cell adhesion $[14,22]$. The pancreatic $\beta$-cells are also known to bind arginine-glycine-aspartic acid (RGD) sequences on FN by expressing integrin $a_{3} \beta_{1}, a_{5} \beta_{1}$, and $a_{v} \beta_{3}$, which promotes cell survival [23, 24]. The density of these integrins decreases after enzymatic degradation of the islet basement membrane during isolation, which negatively affects islet cells function [19]. 
Reintroducing ECM molecules has been reported to improve $\beta$-cell survival and function. For example, culturing $\beta$-cells on ECM secreted by bovine corneal endothelial cells improves insulin release [25], islet survival, and proliferation rate of cultured $\beta$-cells [26]. Furthermore, purified individual ECM molecules such as collagen type I (Col1), Col4, LN and FN can increase the adhesion and insulin release of $\beta$-cells $[14,22,27,28]$. Although previous studies have provided valuable insights, they were limited by conventional culturing of $\beta$-cells, which can negatively affect their metabolism and functionality [29, 30]. Microcontact printing can be used to create arrays of specific patterns of proteins on a non-cell adherent material surface with high spatial resolution in a highly reproducible manner. By carefully selecting a specific pattern using a particular ECM molecule, or a mixture of ECM molecules, one is able to design a cell culture substrate allowing for studying $\beta$-cell behavior and functionality on a micrometer scale. This microcontact printing platform can provide in depth insight in the role of ECM proteins on $\beta$ cells. Here, we set-out to study the influence of circular spatially patterned isletssized ECM patches on the function and survival of $\beta$-cells. We hypothesize that $\beta$ cells function and viability can be improved by particular ECM proteins, and that growing cells in three dimensional aggregates in the presence of the proteins further improves their endocrine function. Specifically, we explored covalent microcontact printing of FN, Col4, and LN111 on glucose responsiveness of INS-1E rat insulinoma cells. We confirm that uniform coatings of FN and Col4 as well as microcontact printed patterned coatings significantly increases the insulin secretion of INS-1E cells as compared to non-coated surfaces. Interestingly, LN111 adversely affected insulin secretion in comparison to the non-coated substrate.

Notably, all microcontact printed coatings outperformed monolayer coatings in terms of INS-1E insulin secretion. Remarkably, micropatterning revealed to be an effective manner to engineer islet-like cell clusters via self-organization of INS-1E cells. Together this study provides valuable insights in the engineering of islet-like microenvironments, which are expected to be of significant importance in successful engineering of islets to remedy type 1 diabetes. 


\section{Materials and methods}

\section{Fabrication of a microcontact printed substrate}

To fabricate uniform patterns of ECM molecules on a substrate, we modified the method published by Mendelsohn et al [31]. In short, a silicon based master wafer was prepared by micro photolithography to create a pattern of UV crosslinked SU8. The pattern's design consists of 2500 circular spots $/ \mathrm{cm}^{2}$, each spot having a diameter of $100 \mu \mathrm{m}$. PDMS stamps with a thickness of 1-1.5 mm were prepared by negative replica molding a master wafer using a 1:10 w/w mixture of Sylgaard 184 elastomer (Dow Corning, USA). The stamps were treated by plasma oxidization (Plasma-Preb II plasma ether, SPI supplies, USA) at an oxygen pressure of 1.0 bar and at $40 \mathrm{~mA}$ for $20 \mathrm{~s}$ to increase the hydrophilicity of PDMS and to clean the surface from organic contaminants. Subsequently, the stamps were inked with 50 $\mu \mathrm{l}$ of solubilized FN, Col4, or LN111 (100 $\mu \mathrm{g} / \mathrm{ml}$ in PBS) labeled with the fluorophore DyLight 488 or 549 NHS Ester (Thermo Fisher Scientific). The stamps were washed with Milli-Q water and were dried under nitrogen flow. Afterwards, the excess inking solution of FN, Col4, or LN111 was removed from the stamp and dried under a stream of $\mathrm{N}_{2}$. PDMS films with a thickness of 3-5 mm were created by casting on flat silicon wafers to serve as inert cell culture substrates. The PDMS stamp and substrate was treated by plasma oxidization at an oxygen pressure of 1.0 bar and at $40 \mathrm{~mA}$ for $20 \mathrm{~s}$ and $90 \mathrm{~s}$, respectively. The micropatterned stamp was placed on the flat PDMS substrate for $20 \mathrm{~min}$. A $25 \mathrm{gr} / \mathrm{cm}^{2}$ weight was placed on top of the stamp to ensure homogeneous transferring of the molecules on the substrate. Subsequently, the non-printed areas of the substrate were blocked via incubation of $10 \% \mathrm{w} / \mathrm{v}$ Pluronic F108 in PBS for 3 hours at room temperature. Substrates were then gently washed with PBS, disinfected with $70 \%$ ethanol for 2 minutes, washed with sterile PBS, and imaged using fluorescence microscopy. 
Using this stamp, $20 \%$ of the surface of the substrate $\left(1.9 \times 10^{7} \mu \mathrm{m}^{2}\right)$ was microcontact printed with ECM molecules.

\section{Cell culture}

INS-1E rat insulinoma cells were cultured in RPMI 1640 medium with $2.05 \mathrm{mM}$ L-glutamine (Invitrogen) supplemented with 5\% FBS, 100U/ml penicillin (GIBCO, Bleiswijk, the Netherlands), $100 \mathrm{U} / \mathrm{ml}$ streptomycin (Lonza, Verviers, Belgium), 10 mM HEPES, $1 \mathrm{mM}$ sodium pyruvate, and $50 \mu \mathrm{M}$ freshly added $\beta$-mercaptoethanol. Cells were incubated in humidified air $(5 \% \mathrm{CO} 2)$ at $37^{\circ} \mathrm{C}$.

\section{Cell seeding density}

In order to have an equal cell density on the micropatterned substrates and tissue culture plastic (TCP), $40 \times 10^{4}$ INS-1E cells $/ \mathrm{cm}^{2}$ were seeded on microcontact printed FN, Col4, and LN111 patterned PDMS, while $10 \times 10^{4}$ INS-1E cells/cm ${ }^{2}$ were seeded on uniformly coated TCP plates and non-coated TCP plates (control group). After 6 hours, the samples were supplemented with fresh medium. The cell seeding density was determined by quantifying the DNA content using a Quant-iT picogreen dsDNA assay kit (Invitrogen) according to the manufacturer's protocol.

\section{Morphology of the cells}

To study the effect of the ECM molecules on the INS-1E cell morphology, samples were fixed in 4\% $(\mathrm{w} / \mathrm{v})$ paraformaldehyde in PBS for 1 hour at room temperature after 1, 3, and 7 days of culture. Subsequently, samples were dehydrated using an increasing concentration series from $70 \%$ to $100 \%$ ethanol for 30 minutes in each step. Afterwards, samples were critically point dried (CPD 030, BAL-TEC), gold coated by a gold sputter coater (Cressington UK) and imaged using scanning electron microscopy (XL30 ESEM-FEG, Philips, FEI, The Netherlands). To further assess cell and aggregate morphology, samples of each condition were fixed in 4\% $(\mathrm{w} / \mathrm{v})$ paraformaldehyde in PBS for 1 hour at room temperature after 3 and 7 of culture and imaged using bright-field microscopy (Nikon TE300, Japan). 


\section{Cell viability}

A two-color fluorescent LIVE/DEAD viability/cytotoxicity Kit (Invitrogen, Carlsbad, CA) was used to evaluate the viability of INS-1E cells at each time point. The assay was performed on cells cultured on uniformly coated TCP plates, and microcontact printed PDMS samples functionalized with ECM molecules after 3 and 7 days. Green fluorescent Calcein-AM indicates live cells by labeling intracellular esterase, and red fluorescent Ethidium homodimer-1 indicates dead cells by complexation to DNA, which can only occur when plasma membranes are damaged. Samples were imaged using fluorescence microscopy (Olympus 1X71, Japan). Viability was quantified as percentage of viable cells per total cell number (Figure 2).

\section{Glucose stimulated insulin secretion test}

Glucose-stimulated insulin secretion (GSIS) tests were performed on INS-1E cells after 3 and 7 days of culture ( $n=3$ /condition). Each sample was pre-incubated for 1 hour in a glucose-free incubation buffer consisting of a modified Krebs-Ringer bicarbonate buffer with HEPES and $10 \mathrm{mM}$ theophylline $(\mathrm{KRBH})$ containing 115 mmol/L NaCl, 5 mmol/L KCL, 24 mmol/L NaHCO3, $2.2 \mathrm{mmol} / \mathrm{L} \mathrm{CaCl}_{2}, 1 \mathrm{mmol} / \mathrm{L}$ $\mathrm{MgCl}_{2}, 20 \mathrm{mmol} / \mathrm{L}$ HEPES, and $2 \mathrm{~g} / \mathrm{L}$ human serum albumin at $\mathrm{pH}$ 7.4. Subsequently, samples were incubated with three consecutive steps consisting of low glucose (1.6 mmol/L), high glucose (16.7 $\mathrm{mmol} / \mathrm{L})$, and another low glucose (1.6 mmol/L) in $\mathrm{KRBH}$ buffer for $90 \mathrm{~min}$ at $37{ }^{\circ} \mathrm{C}$. The media samples were collected after each incubation step and analyzed for insulin content using an ELISA assay (Mercodia, Uppsala, Sweden) according to the manufacturer's protocol. Finally, cell seeding densities were determined via DNA quantification as described above. The DNA quantities were used to normalize the released insulin.

\section{Statistical analysis}

Results are presented as mean \pm standard deviation. Statistical analysis was performed using a Student's T-test, One-way ANOVA and a least-significant 
difference (LSD) multiple comparison test by application of SPSS statistic software (Chicago, USA). Statistical significance was considered at $\mathrm{P}<0.05$.

\section{Results}

\section{Microcontact printing of extracellular matrix molecules}

To evaluate the effect of ECM molecules on function, viability and morphology of the INS-1E cells, three different ECM molecules were applied as uniform or microcontact printed coatings (Figure 1A). Specifically, FN, Col4, and LN111 were selected due to their abundance in islets. Cell cultures on non-coated TCP plates served as negative controls. Fluorescence microscopy indicated that on the patterned substrates $100 \mu \mathrm{m}$ diameter circular spots were uniformly coated with FN, Col4, or LN111 (Figure 1B and C). Evaluation of the fluorescent intensity indicated that the covalently immobilized FN, Col4, and LN111 are stable for at least 7 days after microcontact printing (Figure 1D). After seeding the INS-1E cells on microcontact printed substrates, the cells spontaneously self-assembled into uniform cell clusters forming well rounded aggregates (Figure 1E).

Since cell-cell interactions can play an important role in $\beta$-cell function we ensured that identical cell concentrations for each condition were used. Since only $20 \%$ of the PDMS surface $\left(1.9 \times 10^{7} \mu \mathrm{m}^{2}\right.$ of $\left.1 \mathrm{~cm}^{2}\right)$ was treated with microcontact printing of circular ECM spots, cells that were unable to adhere to the microprinted spots of ECM were removed $6 \mathrm{~h}$ after seeding when the medium was refreshed. In order to assure that the same number of cells on microcontact printed, non-coated and uniformly coated samples was seeded, $40 \times 10^{4}$ INS-1E cells $/ \mathrm{cm}^{2}$ on microcontact printed FN, Col4 and LN111 substrates, and $10 \times 10^{4}$ INS-1E cells/cm ${ }^{2}$ on non-coated, and uniformly coated samples was used (Figure 1F). These numbers were verified after a series of cell seeding optimization steps combined with determining the total amount of DNA present after cell culture. 




Patterned master

B

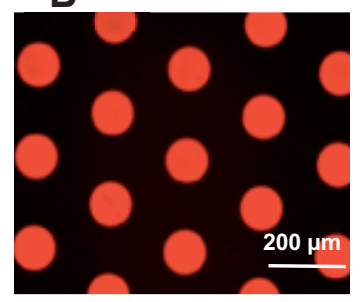

PDMS molding
Coating PDMS stamp with ECM
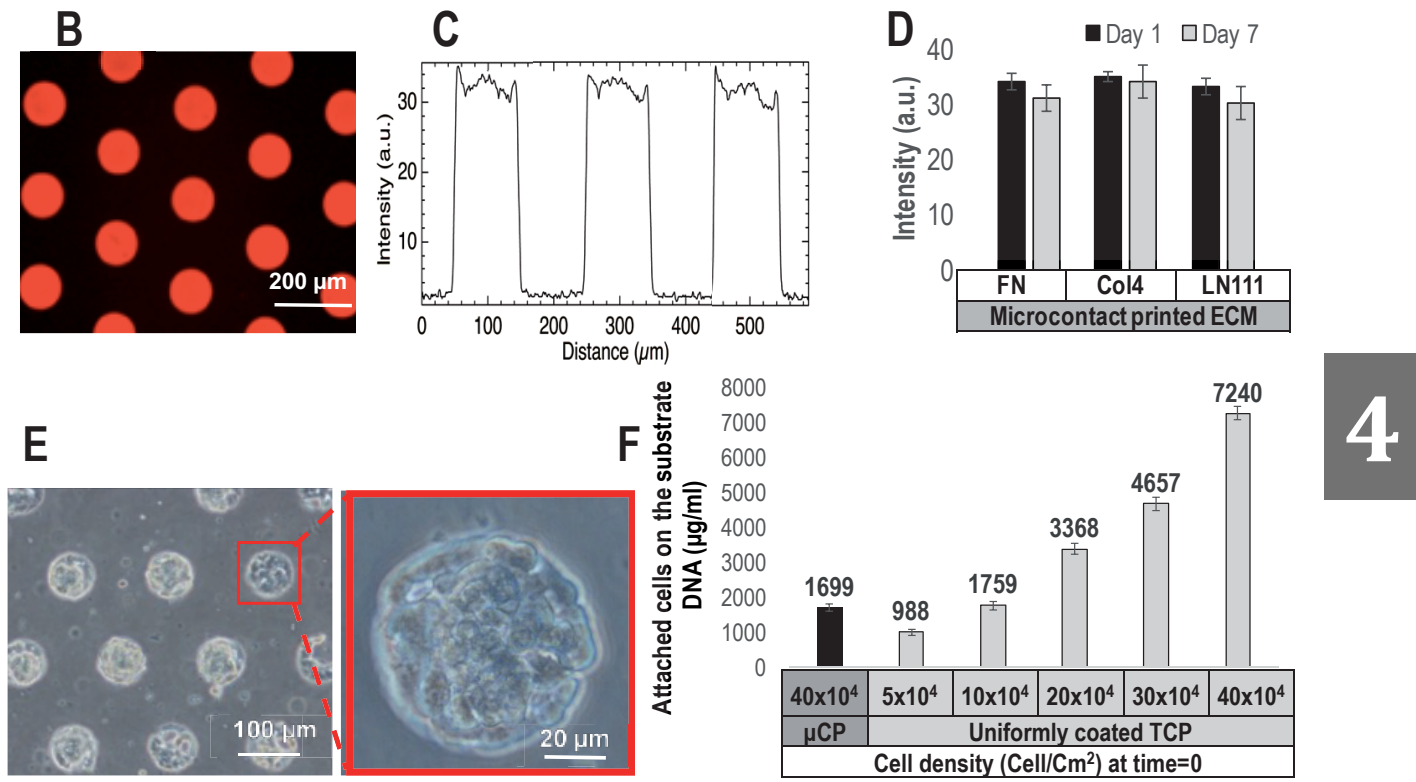

Figure 1. Fabrication of cell seeded ECM microcontact printed arrays. A) Schematic outline of the creation of the microcontact printed ECM arrays. B) Homogenous transfer of fluorescently labelled fibronectin validated proper transfer of ECM proteins. C) Homogeneity of the microcontact printed spots was confirmed using fluorescence intensity distribution quantification. D) Consecutive fluorescent intensity measurements of the microcontact printed fibronectin (FN), collagen-IV (Col4) and laminin-111 (LN111) demonstrated that the patterns were stable in cell culture medium at $37^{\circ} \mathrm{C}$ for at least 7 days. E) INS-1E cells were able to grow on microcontact printed spots, but not on non-printed areas. F) An equal cell density between patterned and non-patterned, or uniformly coated substrates was determined using DNA quantification.

\section{Viability of INS-1E cells on micropatterns of ECM}

To validate the cytocompatibility of INS-1E cells on ECM micropatterned substrates, the viability of INS-1E cells cultured on conventional ECM coatings, and 
microcontact printed ECM spots was investigated after one and seven days (Figure 2 A). Uncoated TCP was used as a negative control. Live/dead staining revealed a cell viability of $>90 \%$ for all microcontact printed samples after one and seven days of culture. Notably, the percentage of live cells on microcontact printed ECM spots was consistently higher than those of INS-1E cells cultured on either uniformly coated, or TCP plates after 7 days (Figure 2B).

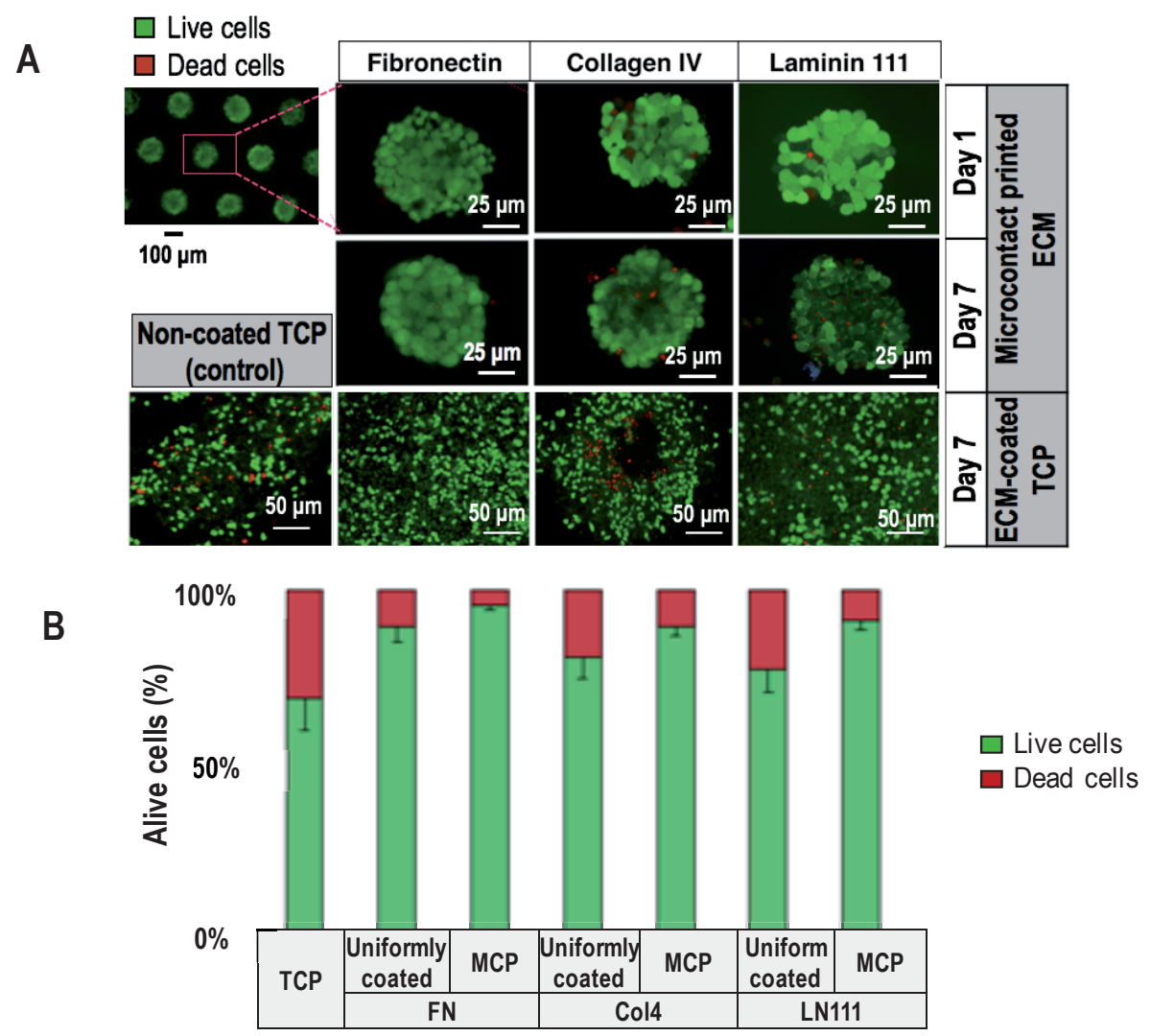

Figure 2. Evaluation of the cell viability in the presence of fibronectin, collagen IV, and laminin 111 on a uniformly coated TCP plate and microcontact printed PDMS. A) Live/Dead staining of INS-1E cells at day 1 and 7, B) Imaged-based semi-quantification of the amount of live and dead cells after 7 days 


\section{Microcontact printed ECM spots induce INS-1E cells to self-assemble in islet-sized aggregates}

We assessed the effect of microcontact printed FN, Col4, and LN111 on INS-1E cell morphology using SEM after 1, 3, and 7 days of culture. The results indicated that the cells were able to adhere homogeneously to the printed spots. Subsequently, the cells grew first in a monolayer at day 1, and then formed a multilayer at day 3, and self-assembled into 3D islet-sized micro-aggregates at day 7 (Figure 3A). The aggregates had a width of $72 \pm 10,76 \pm 9$ and $75 \pm 12 \mu \mathrm{m}$, and a height of $23.3 \pm 3.6,38.7 \pm 4,8$ and $41.5 \pm 4.2 \mu \mathrm{m}$ for cells cultured respectively on FN, Col4, and LN111 microcontact printed substrates (Figure 3B and C). In order to determine the number of INS-1E cells, which had adhered to the uniformly coated and microcontact printed samples after 3 and 7 days, we determined the total amount of DNA after culture. Although all samples had initially been seeded with the same number of cells at the start of the experiment we found that the DNA content on the LN111 microcontact printed samples was higher than those of the Col4 and FN printed samples (Figure 3D). In detail, the DNA content for control samples as well as uniform coated FN, Col4 and Lm respectively were, $15400 \pm 3000,25100 \pm 4500,35300 \pm 7000$, and $43400 \pm$ $9000 \mu \mathrm{g} / \mathrm{ml}$ at day 7. Moreover, the DNA content for microcontact printed FN, Col4 and Laminin respectively were $20600 \pm 3000,28200 \pm 5000$, and $380200 \pm 5700$ $\mu \mathrm{g} / \mathrm{ml}$ at day 7 (Figure 3D) 

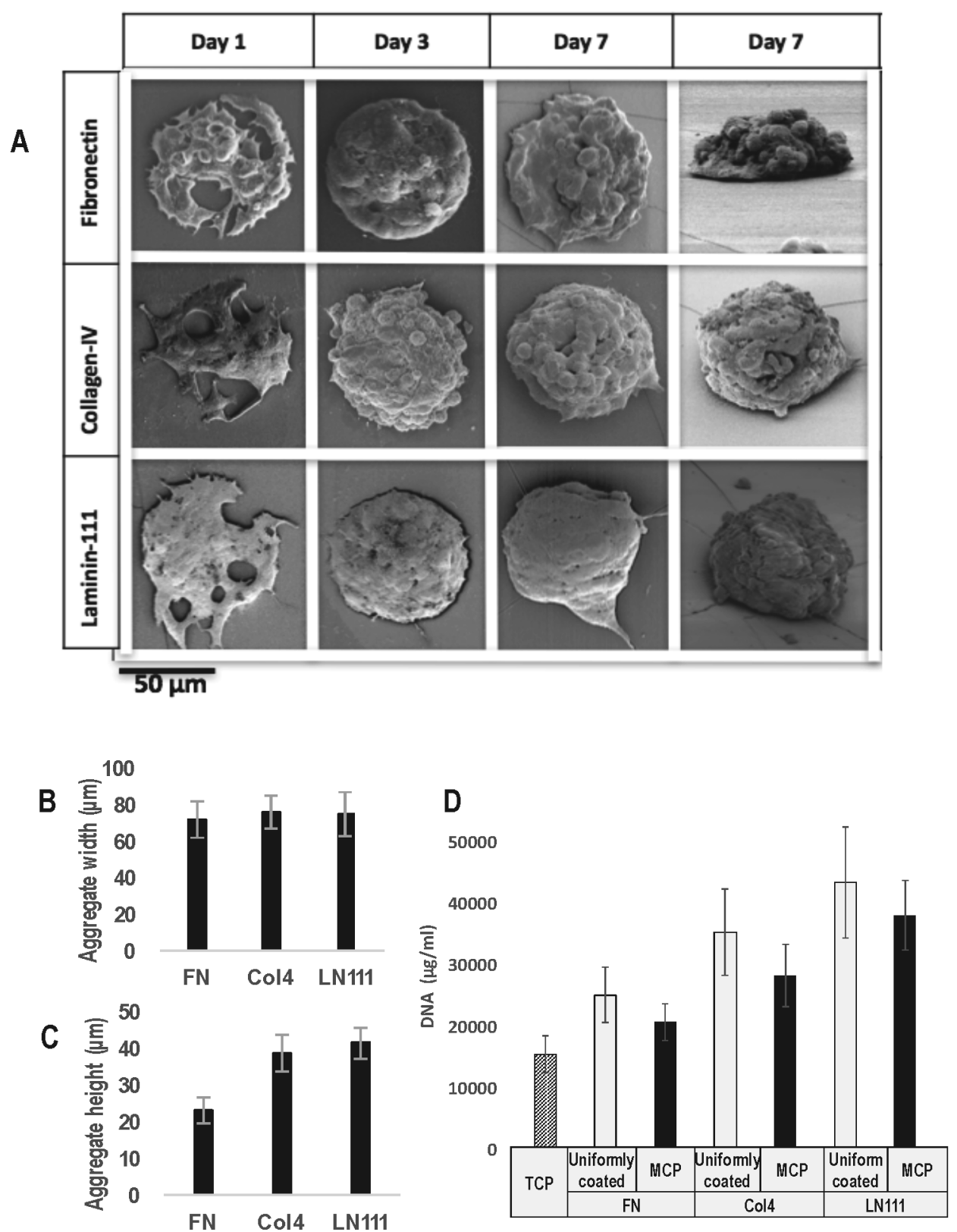

Figure 3. A) Electron micrographs of INS-1E cells cultured on microcontact printed (MCP) fibronectin (FN), collagen-IV (Col4) and laminin-111(LN111) substrates after 1, 3 and 7 days of culture. B) Height of aggregates on FN, Col4, and LN111 microcontact printed substrates after 7 days, C) width of the aggregates on FN, Col4, and LN111 microcontact printed substrates, D) The total cell number of INS-1E cells on microcontact printed and coated FN, Col4, and LN111 after 7 days of culture. 


\section{Glucose responsiveness of INS1E cell clusters}

Glucose stimulated insulin secretion of INS-1E cells was assessed in INS-1E cells cultured on microcontact printed PDMS substrates, uniformly coated TCP plates and non-coated TCP plates after 7 days. Insulin secretion was significantly higher in cells cultured on both uniform coated as well as microcontact printed Col4 (13.9 \pm 3.5 and $17.9 \pm 4.8$ picomol $/ \mu \mathrm{g}$ DNA respectively) and FN (11.6 \pm 1.5 and 15.5 $\pm 2 \mathrm{picomol} / \mu \mathrm{g}$ DNA respectively) as compared on TCP plates alone $(6.2 \pm 1.0$ picomol/ $\mu \mathrm{g}$ DNA). In contrast, cells cultured on both uniform coated as well as microcontact printed LN111 showed a strongly reduced insulin secretion (1.2 \pm 0.1 and $3.8 \pm 0.9 \mathrm{picomol} / \mu \mathrm{g}$ DNA, respectively) as compared to the non-coated control group. Regardless of which ECM was used as coating, the insulin secretion was significantly higher in cells cultured on microcontact printed substrates as compared to uniformly coated samples.

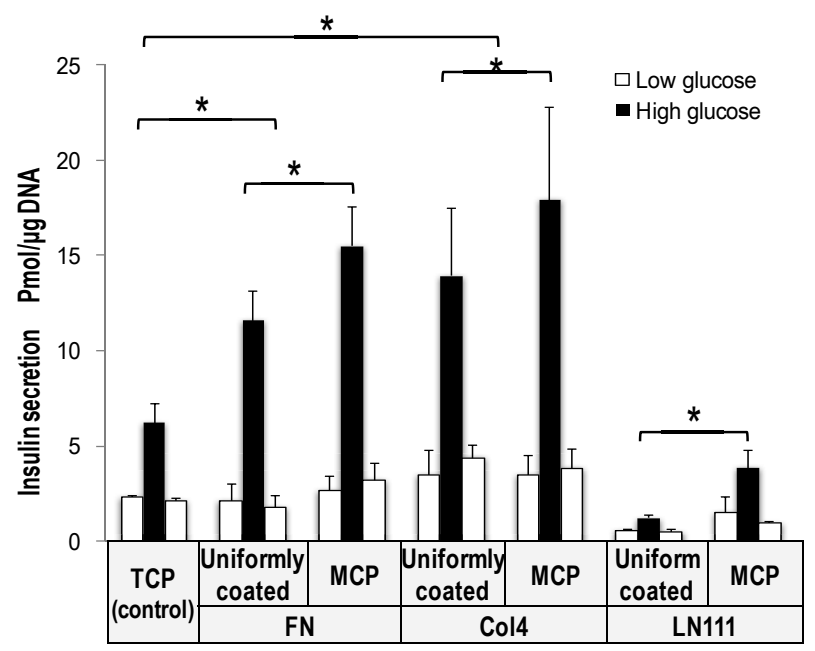

Figure 4. Glucose stimulated insulin secretion test of INS-1E cells on uniformly coated and microcontact printed (MCP) fibronectin(FN), collagen-IV (Col4) and laminin-111(LN111) after 7 days cell culturing. 


\section{Discussion}

ECM molecules influence the cellular function, survival, morphology, proliferation, and differentiation. Isolation of islets from their native microenvironment using enzymatic digestion disrupts the interaction between the islet cells and ECM molecules such as FN, Col4 and Lm [1, 2]. Introducing cell-ECM interaction using an ECM coating has the potential to improve $\beta$-cell function. However, studies exploring various methods to apply ECM molecules in islet or $\beta$ cell cultures are largely missing.

This study demonstrated the effect of FN, Col4, and LN111 on INS-1E cells using microcontact printing. Microcontact printing provides an easy platform to study the interaction between the cells and ECM proteins on a functionalized surface of PDMS to which cells normally don't adhere due to its hydrophobic properties. Recently, many studies focused on minimizing the drawbacks of microcontact printing on soft pliable surfaces, such as ink-transfer issues and stamp deformation. Based on these studies we created homogeneous and uniform patterns of FN, Col4 or LN111 circular spots with a diameter of $100 \mu \mathrm{m}$ on the surface of PDMS. Consecutive fluorescent intensity measurements of microcontact printed FN, Col4 and Lm patterns demonstrated that proteins were homogeneously transferred and remained stable in medium at $37^{\circ} \mathrm{C}$ for at least 7 days. After cell seeding and prolonged culture the micropatterned ECM spots enabled self-assembly of reproducible similarly shaped islet-like clusters from INS 1E cells.

Previous studies indicated that cell-cell contact and islet size are important for the function and survival of $\beta$-cells. Some studies indicate that relatively large aggregates $(>150 \mu \mathrm{m})$ quickly lead to apoptosis of $\beta$-cells, ultimately resulting in loss of endocrine function [32, 33]. Moreover, while $\beta$-cell clusters of $>100 \mu \mathrm{m}$ do not demonstrate a significant amount of cell loss by apoptosis, they still were 
reported to secrete less insulin compared to even smaller, $<100 \mu \mathrm{m}, \beta$-cell clusters [34].

Our results revealed that the FN and Col4 coated spots prepared by microcontact printing improve the function and survival of the INS-1E cells compared to conventional suspension cultures. We observed that the proliferation rate of INS1E cells cultured on LN111 was considerably higher than on FN and Col4. In contrast, the glucose responsiveness of cells in the presence of LN111 was significantly less than FN and Col4 as can be seen in figure 4. This suggests that LN111 plays an important role in cell proliferation rather than enhancing the endocrine function. Notably, regardless of which ECM was used as coating, the insulin secretion was significantly higher in cells cultured on microcontact printed substrates as compared to uniformly coated substrates. The intensified cell-cell contact, due to the self-assembly of INS-1E cells into islet-like cell clusters, on the microcontact printed spots could potentially explain this consistent observation.

Other studies support our results by indicating that FN and Col4 can improve the insulin secretion of $\beta$-cells $[35,36]$. Moreover, it has been reported that FN increases the insulin secretion at a same level of coated RGD to a substrate, [37] which indicates the importance of the RGD sequence in the molecular structure of FN. Some studies indicated the role of laminin 111 on survival, differentiation and insulin gene expression of the islets [38] [39]. However, information on the effect of different laminins has remained scarce and the effect of LN111 on insulin release until now remains unknown.

It is of note that we observed a positive effect of FN, Col4, and LN111 on cell viability, which could not be achieved via the relatively low survival rate of cells on uncoated substrates [13]. This observation might be obviated in different culture systems with higher basic cell survival rates. For example, encapsulation of the MIN6B1 cells in hydrogels without ECM molecules can result in high survival rates $[13,40]$. 
Overall, microcontact printing provides a great potential in the development of scaffolds for treatment of type 1 diabetes. By mimicking the size and round structure of islets significant improvements in the function of $\beta$-cells may be achieved.

Comparing the morphology of the aggregates indicated that the cells on microcontact printed spots of LN111 appear more compacted than cells on FN, Col4 which might negatively affect the insulin release of the cells. There is no study regarding the effect of laminins on compaction of $\beta$-cells. However, some studies indicated that extracellular stimuli as well as cell-cell adhesion molecules regulate cell signaling which are involved in morphological changes of epithelial cells [41].

\section{Conclusions}

We demonstrated that FN, Col4 and LN111 are homogenously printable by microcontact printing on a flat PDMS surface. Our results indicated that microcontact printing provides an effective method to reproducibly enable the selfassembly of cells into microaggregated cell clusters. Moreover, we revealed that microcontact printing induced self-assembly of cells which improved the function and survival of the INS-1E cells as compared to conventional cultures. Cells cultured on conventionally coated surfaces do not present a natural cellular morphology and functionally as compared to cells in their native microenvironment. Here, we reported that microcontact printing can be used to enable spontaneous selfassembly of cells to evaluate further the effect of cell-cell and ECM-cell interaction in a more biomimetic microenvironment. Consequently, this approach holds great potential for applications in tissue regeneration and drug discovery, stem cell research, and many other cell-based analyses and devices. Specifically, we propose that microcontact printing could aid in the development of next-generation scaffolds for islet transplantation strategies to treat type 1 diabetes. 
In this study, we show a novel method to generate $\beta$-cell clusters of 50-100 $\mu \mathrm{m}$ in a highly reproducible and controlled manner using microcontact printed circular spots. As such, microcontact printing offers an alternative platform to study the cluster size dependent $\beta$-cells function and survival. Advantageously, this method inherently enables the study of ECM coated substrates, a feat that has remained difficult to control in current platforms such as hanging drops and microwells. In addition, it is likely that by varying the microcontact printing spot size and seeding density a wide range of cell cluster sizes could be achieved, which is expected to be of value to other fields that rely on three dimensional cell culture.

\section{Acknowledgments}

We acknowledge financial support from the Diabetes Cell Therapy Initiative

(DCTI) FES 2009 program LSH-DCTI including the Dutch Diabetes Research Foundation (DF). 


\section{References}

1. Cross, S.E. et al. (2008) Collagenase penetrates human pancreatic islets following standard intraductal administration. Transplantation 86 (7), 907-911.

2. Rosenberg, L. et al. (1999) Structural and functional changes resulting from islet isolation lead to islet cell death. Surgery 126 (2), 393-398.

3. Thivolet, C. et al. (1985) Morphological and functional effects of extracellular matrix on pancreatic islet cell cultures. Experimental cell research 159 (2), 313322.

4. Montesano, R. et al. (1983) Collagen Matrix Promotes Pancreatic Endocrine Cell. The Journal of Cell Biology 97, 935-939.

5. Jiang, F.-X. et al. (1999) Laminin-1 promotes differentiation of fetal mouse pancreatic beta-cells. Diabetes 48 (4), 722-730.

6. Peiris, H. et al. (2014) The $\beta$-cell/EC axis: how do islet cells talk to each other? Diabetes 63 (1), 3-11.

7. Rondas, D. et al. (2012) Novel mechanistic link between focal adhesion remodeling and glucose-stimulated insulin secretion. Journal of Biological Chemistry 287 (4), 2423-2436.

8. Daoud, J. et al. (2010) The effect of extracellular matrix components on the preservation of human islet function in vitro. Biomaterials 31 (7), 1676-1682.

9. Boretti, M.I. and Gooch, K.J. (2008) Effect of extracellular matrix and 3D morphogenesis on islet hormone gene expression by Ngn3-infected mouse pancreatic ductal epithelial cells. Tissue Engineering Part A 14 (12), 1927-1937.

10. Edamura, K. et al. (2003) BRIEF COMMUNICATION: Effect of Adhesion or Collagen Molecules on Cell Attachment, Insulin Secretion, and Glucose Responsiveness in the Cultured Adult Porcine Endocrine Pancreas: A Preliminary Study. Cell transplantation 12 (4), 439-446.

11. Ryschich, E. et al. (2009) Promotion of tumor cell migration by extracellular matrix proteins in human pancreatic cancer. Pancreas 38 (7), 804-810.

12. Beattie, G.M. et al. (1996) Regulation of proliferation and differentiation of human fetal pancreatic islet cells by extracellular matrix, hepatocyte growth factor, and cell-cell contact. Diabetes 45 (9), 1223-1228.

13. Weber, L.M. et al. (2008) Cell-matrix interactions improve $\beta$-cell survival and insulin secretion in three-dimensional culture. Tissue Engineering Part A 14 (12), 1959-1968.

14. Kaido, T. et al. (2004) Regulation of human $\beta$-cell adhesion, motility, and insulin secretion by collagen IV and its receptor a1ß1. Journal of Biological Chemistry 279 (51), 53762-53769. 
15. Hamamoto, Y. et al. (2003) Beneficial effect of pretreatment of islets with fibronectin on glucose tolerance after islet transplantation. Hormone and metabolic research 35 (08), 460-465.

16. Jiang, F.-X. et al. (2002) Distinct distribution of laminin and its integrin receptors in the pancreas. Journal of Histochemistry \& Cytochemistry 50 (12), $1625-1632$.

17. Mandrup-Poulsen, T. (2001) beta-cell apoptosis: stimuli and signaling. Diabetes 50 (suppl 1), S58.

18. Lucas-Clerc, C. et al. (1993) Long-term culture of human pancreatic islets in an extracellular matrix: morphological and metabolic effects. Molecular and cellular endocrinology 94 (1), 9-20.

19. Wang, R.N. et al. (1999) Characterization of integrin expression in islets isolated from hamster, canine, porcine, and human pancreas. Journal of Histochemistry \& Cytochemistry 47 (4), 499-506.

20. Cirulli, V. et al. (2000) Expression and Function of $\operatorname{av\beta 3}$ and avß5 Integrins in the Developing Pancreas. The Journal of cell biology 150 (6), 1445-1460.

21. Kantengwa, S. et al. (1997) Identification and characterization of a $3 \beta 1$ integrin on primary and transformed rat islet cells. Experimental cell research 237 (2), 394402.

22. Kaido, T. et al. (2004) av-Integrin utilization in human $\beta$-cell adhesion, spreading, and motility. Journal of Biological Chemistry 279 (17), 17731-17737.

23. Stendahl, J.C. et al. (2009) Extracellular matrix in pancreatic islets: relevance to scaffold design and transplantation. Cell transplantation 18 (1), 1-12.

24. Pinkse, G.G. et al. (2006) Integrin signaling via RGD peptides and anti- $\beta 1$ antibodies confers resistance to apoptosis in islets of Langerhans. Diabetes 55 (2), 312-317.

25. BEATTIE, G.M. et al. (1991) Functional impact of attachment and purification in the short term culture of human pancreatic islets. The Journal of Clinical Endocrinology \& Metabolism 73 (1), 93-98.

26. Schuppin, G. et al. (1993) Replication of adult pancreatic-beta cells cultured on bovine corneal endothelial cell extracellular matrix. In Vitro Cellular \& Developmental Biology-Animal 29 (4), 339-344.

27. Hammar, E.B. et al. (2005) Activation of NF- $\kappa B$ by extracellular matrix is involved in spreading and glucose-stimulated insulin secretion of pancreatic beta cells. Journal of Biological Chemistry 280 (34), 30630-30637.

28. Nagata, N. et al. (2001) Evaluation of insulin secretion of isolated rat islets cultured in extracellular matrix. Cell transplantation 10 (4-1), 447-451. 
29. Edmondson, R. et al. (2014) Three-dimensional cell culture systems and their applications in drug discovery and cell-based biosensors. Assay and drug development technologies 12 (4), 207-218.

30. Abbott, A. (2003) Cell culture: biology's new dimension. Nature 424 (6951), 870-872.

31. Mendelsohn, A.D. et al. (2010) Patterning of Mono-and Multilayered Pancreatic ß-Cell Clusters. Langmuir 26 (12), 9943-9949.

32. MacGregor, R. et al. (2006) Small rat islets are superior to large islets in in vitro function and in transplantation outcomes. American Journal of PhysiologyEndocrinology and Metabolism 290 (5), E771-E779.

33. Lehmann, R. et al. (2007) Superiority of small islets in human islet transplantation. Diabetes 56 (3), 594-603.

34. Nittala, A. et al. (2007) Investigating the role of islet cytoarchitecture in its oscillation using a new $\beta$-cell cluster model. PLoS One 2 (10), e983.

35. Hammar, E. et al. (2004) Extracellular matrix protects pancreatic $\beta$-cells against apoptosis. Diabetes 53 (8), 2034-2041.

36. Langhofer, M. et al. (1993) The matrix secreted by $804 \mathrm{G}$ cells contains lamininrelated components that participate in hemidesmosome assembly in vitro. Journal of Cell Science 105 (3), 753-764.

37. Kuehn, C. et al. (2012) Culturing INS-1 cells on CDPGYIGSR-, RGD-and fibronectin surfaces improves insulin secretion and cell proliferation. Acta biomaterialia 8 (2), 619-626.

38. Jiang, F.-X. et al. (2001) Regulation of laminin 1-induced pancreatic beta-cell differentiation by alpha6 integrin and alpha-dystroglycan. Molecular Medicine 7 (2), 107.

39. Nikolova, G. et al. (2006) The vascular basement membrane: a niche for insulin gene expression and $\beta$ cell proliferation. Developmental cell 10 (3), 397-405.

40. Weber, L.M. and Anseth, K.S. (2008) Hydrogel encapsulation environments functionalized with extracellular matrix interactions increase islet insulin secretion. Matrix Biology 27 (8), 667-673.

41. Wakayama, Y. et al. (2011) EphrinA1-EphA2 signal induces compaction and polarization of Madin-Darby canine kidney cells by inactivating Ezrin through negative regulation of RhoA. Journal of Biological Chemistry 286 (51), 4424344253. 




\title{
Chapter 5
}

Mimicking the islet extracellular matrix niche in microwell islet delivery devices using collagen-IV and laminin 111 leads to improved insulin secretion in human islets

\author{
E. Hadavi \\ J. Leijten \\ P. Jonkheijm \\ M. Engelse \\ E. de Koning \\ A.A. van Apeldoorn \\ H.B.J. Karperien
}




\section{Abstract}

Intrahepatic islet-transplantation is a promising therapy for treatment of type 1 diabetes. During islet isolation, collagenase is used to extract islets from the pancreas, leading to loss of important cell-matrix interactions. Loss of the native pancreatic microenvironment is associated with apoptosis of islet cells, early graft failure, and poor islets function. In short, the degradation of the pancreatic microenvironment limits the clinical success of islet transplantation. The islet extracellular matrix is comprised of a specific combination of collagen, laminin, and fibronectin molecules. Reintroducing these molecules has been shown to boost the function, viability, morphology, and proliferation of $\beta$-cells. In this research, the effect of combinatorial ECM on islet function and survival was investigated. Specifically, thin film microwell array scaffolds made from two distinct biomaterials were coated with fibronectin, collagen type IV, laminin-111, laminin-332 or a combination thereof. We reveal that coatings containing a single type of ECM molecule, e.g. fibronectin or collagen, can improve short term islet function. However, these single proteins do not prevent loss of morphology and subsequent loss of islet function afterwards. In contrast, combining collagen-IV with laminin-111 at a ratio of 8:2 not only improved short term islet function, but also preserved islet structure and islet function on a longer term. This effect was reproducibly shown on poly(ester urethane) and poly(ethylene glycol terephthalate-b-butylene terephthalate) microwell islet delivery devices as well as tissue culture polystyrene. We concluded that bio-functionalization of inert biomaterials regardless of their molecular composition with a specific combination of islet ECM molecules can support and improve islet function over longer time-periods. Our data suggested that creating a biomimetic islet niche by bio-functionalization of biomaterials can significantly improve the endocrine function of $\beta$-cells. The creation of islet mimicking niches in islet delivery devices leads to an improvement of islet function by restoring part of the islet's extracellular matrix in these devices. 


\section{Introduction}

Intrahepatic islet transplantation is a minimally invasive therapy to treat type 1 diabetes by replacing lost $\beta$-cells. This treatment results in insulin independence in $50 \%$ of the transplanted patients for a period of 3 to 5 years [1]. The success of islet transplantation is significantly influenced by the process of islet isolation from the pancreas. Current islet isolation methods result in separation of islets from their surrounding microenvironment by enzymatic digestion of the pancreatic extracellular matrix (ECM). Degradation of the ECM affects not only islet physiology, but also negatively affects insulin gene expression [2], insulin secretion [3], proliferation [4], differentiation [4, 5], adhesion and survival of $\beta$-cell [6-8]. Therefore, degradation, or removal of islets ECM molecules such as collagen (Col), fibronectin (FN) and laminin (LN) during islet isolation can reduce the efficacy of islet transplantation by providing a suboptimal $\beta$-cell microenvironment.

To prevail the problems that are associated with the removal of ECM molecules after islet isolation, several researchers have investigated the addition of a new ECM to isolated islets. Results of these studies revealed the beneficial short term effects of a new ECM on islet function. For instance, the mechanical support and topological arrangement of the islet's endocrine cells were improved in presence of certain ECM molecules. Consequently, insulin secretion and islet survival were enhanced in these studies [9]. More specifically, it was reported that a combination of collagen type I (Col1) and collagen type IV (Col4) improves the insulin secretion of $\beta$-cell [10]. In another study, it was shown that culturing islets in Col1 hydrogels mixed with Col3, Col4 and LN improves the survival of the islets [11]. The ECM molecules secreted by $804 \mathrm{G}$ cells increase the insulin release of $\beta$-cells [12]. The specific sequence of LN511 promotes islet viability as well as insulin release. Moreover, the synergistic beneficial effect of the LRE sequence of LN in combination with Col4 was reported [13]. In addition, some studies focused on the interaction of ECM molecules with integrin and non-integrin receptors that are expressed by 
human islets. Such interactions regulate adhesion, differentiation, insulin secretion and survival of $\beta$-cells. For instance, human $\beta$-cell adhesion, motility and insulin secretion were associated with the interaction of Col4 and its specific integrin receptors $\left(a_{1} \beta_{1}\right)$ [3]. The $\beta$-cells are also known to bind arginine-glycin-aspartic acid (RGD) sequences present in ECM molecules by expressing integrin $a_{3} \beta_{1}, a_{5} \beta_{1}$, and $\mathrm{a}_{\mathrm{v}} \beta_{3}$, and thereby promoting cell survival [7]. Therefore, it is highly important to increase knowledge about the response of human islet cells upon their interaction with ECM molecules.

Previous research has mainly focused on the effect of specific individual ECM proteins on the function and survival of islet cells. However, studies on the combinatorial effects of different ECM molecules at different ratios on islets are lacking. Considering that the islet microenvironment is a complex system and the success of islet transplantation relies on better simulation of the islet microenvironment, a profound investigation of this complexity is necessary. It is important to discover the most effective combination of ECM molecules in a topologically similar microenvironment.

In our study, we used thin film microwell array scaffolds using a poly(ethyleneglycol-terephthalate-polybutylene-terephthalate) (4000PEOT30РBT70) abbreviated as (PEOT-PBT) and a poly(ester urethane) to encapsulate islets. The poly(ester-urethane) was synthesized by reaction of an a, $\omega$-isocyanate end functionalized pre-polyester and an a, $\omega$-hydroxy bis-carbamate (Chapter 3 ). The scaffolds were coated with combinations of FN, Col4 as well as various laminins, and afterwards the glucose responsiveness of human islet inside these devices was investigated. We showed that a combination of laminin-111 (LN111) and Col4 has a reproducible synergistic positive effect on the function of the human islets. Furthermore, the shape and dimensions of the microwell scaffolds used offer a potential advantage to support the three-dimensional structure of islets. Interestingly, the positive effect on endocrine function was independent of the biomaterial used to manufacture the microwell scaffold, and not only the amount 
of insulin secreted by the islets was significantly increased, but also their stimulation index was higher in the presence of Col4 and LN in a ratio of 8:2.

\section{Materials and Methods}

\section{Fabrication of PEOT-PBT and poly(ester urethane) films}

The PEOT-PBT block copolymer is composed of a soft hydrophilic segment poly(ethylene oxide terephthalate) and a hard hydrophobic segment poly(butylene terephthalate) and was produced by Polyvation BV (Groningen, the Netherlands, under the trade name PolyActive $\left.{ }^{\mathrm{TM}}\right)$. PEOT is synthesized using polyethyleneglycol with a molecular weight of 4000, while the weight percentage of PEOT and PBT is kept at 30:70 wt\%, respectively [14]. Films with a thickness of $40 \mu \mathrm{m}$ were created using a solvent casting method. Shortly, PEOT-PBT was dissolved in a 65:35 (w/w) mixture of chloroform (Merck, Darmstadt, Germany) and 1,1,1,3,3,3-hexafluoro-2-isopropanol (Biosolve, Valkenswaard, the Netherlands) at a concentration of $15 \mathrm{wt} \%$. After casting the polymer on a silicon wafer at room temperature, a nitrogen flow was applied for 4 hours to dry the film. Residual solvent was removed by immersing the polymer film in ethanol. Complete evaporation of residual solvents was achieved by placing the films in a vacuum oven (Heraeus, Hanau, Germany) at $30^{\circ} \mathrm{C}$ for 3 days. The same protocol was applied to fabricate poly(ester urethane) (Polyganics BV Groningen, the Netherlands) films with a thickness of $40 \mu \mathrm{m}$ from a $5 \mathrm{wt} \%$ polymer solution in chloroform. The poly(ester-urethane) was synthesized by reaction of an a, $\omega$-isocyanate end functionalized pre-polyester and an a,w-hydroxy bis-carbamate (Chapter 3).

\section{Preparation of PEOT-PBT and poly(ester urethane) microwell scaffolds}

Micro-back thermoforming was applied to manufacture microwell scaffolds with a three-dimensional microwell structure as was reported before [15]. The backing 
film materials used for PEOT-PBT, and poly(ester urethane) microwell scaffolds consisted of polyethylene (560 $\mu \mathrm{m}$ thickness), and paraffin (540 $\mu \mathrm{m}$ thickness) placed on top of a $20 \mu \mathrm{m}$ thick polyethylene film, respectively. For the poly(ester urethane) microwell scaffold, the initial mold pressure was set at $30 \mathrm{kN}$ at $25^{\circ} \mathrm{C}$, after which the temperature was gradually increased over time. During this process the pressure was stepwise increased at $30^{\circ} \mathrm{C}, 33^{\circ} \mathrm{C}$ and $41^{\circ} \mathrm{C}$ to $40 \mathrm{kN}, 50 \mathrm{kN}$ and $60 \mathrm{kN}$, respectively. The pressure was released as soon as the temperature reached $41^{\circ} \mathrm{C}$, followed by immersion in ice-cold ethanol for $15 \mathrm{~min}$. Subsequently, the films were gently peeled off their molds. Residual traces of paraffin were removed by immersing the microwells in xylene for 1 min followed by 5 times rinsing with $70 \%$ ethanol and then water. Fabrication of PEOT-PBT microwell scaffolds was done in a slightly different way. In short, the mold pressure was set at $40 \mathrm{kN}$ at $30^{\circ} \mathrm{C}$. The pressure was kept constant at $40 \mathrm{kN}$, while the temperature was increased to $85^{\circ} \mathrm{C}$. At $85^{\circ} \mathrm{C}$, the pressure was increased to $65 \mathrm{kN}$, and directly thereafter the temperature was set to $20^{\circ} \mathrm{C}$. As soon as the press device was cooled to $20^{\circ} \mathrm{C}$, the pressure was released. Microwell films were then placed in 70\% ethanol for 15 min at room temperature before removing the scaffold from the mold. Before seeding the islets the microwell scaffolds were disinfected by immersion in $70 \%$ ethanol overnight. The dimensions of individual microwells in the PEOT-PBT and poly(ester urethane) films were determined by measuring 10 random microwells of every third polymer scaffold manufactured.

\section{Immobilization of ECM molecules on culture substrates}

The thin-film PEOT-PBT and poly(ester urethane) microwell array devices were exposed to oxygen plasma at an oxygen pressure of $1.0 \mathrm{bar}$, at $40 \mathrm{~mA}$, and 200 mTorr for 10 and 20 seconds, respectively. The oxygen plasma treatment was used to modify the surface chemistry of the microwell scaffold, and generate aldehyde and epoxide groups that can react with molecules containing free amine groups present in the ECM proteins. Subsequently, microwell scaffolds and tissue culture 
polystyrene plates were coated with $10 \mu \mathrm{g} / \mathrm{ml}$ of ECM protein at room temperature for 2 hours. The ECM proteins include FN, Col4, LN111 (Merck, the Netherlands), LN332 (Biolamina, Sweden), a mixture of LN111, 211, 121, 311, 411 and 511 (Merck, the Netherlands), and pairwise combinations of FN, Col4 and LN at ratios of $5: 5,2: 8$ and 8:2. After three times rinsing in PBS, to remove any unbound proteins, the scaffolds were placed in cell culture inserts and transferred to ultralow-attachment cell culture plates (Corning, USA).

\section{Primary human islet cultures}

Human islets of Langerhans were isolated from pancreas tissue obtained from organ donors (Human Islet Isolation Laboratory, Leiden University Medical Center, Leiden, The Netherlands). Human donor islets were used if not deemed suitable for clinical islet transplantation, and if informed consent was given for research purposes according to national law and regulations. Isolated human donor islets were cultured in CMRL-1066 culture medium (Cellgro, Mediatech, VA, USA) containing $5.5 \mathrm{mM}$ D-glucose, supplemented with 10\% FBS, 100U/ml penicillin, $100 \mathrm{mg} / \mathrm{ml}$ streptomycin, $2 \mathrm{mM}$ L-glutamine, $10 \mathrm{mM} \mathrm{HEPES}$, and $1.2 \mathrm{mg} / \mathrm{ml}$ nicotinamide.

Human islets from two donors (49 year old male, and a 75 years old female) were carefully handpicked and then 50 islets seeded per microwell scaffold and per well on tissue culture plates $(n=3)$. Medium was refreshed every other day. Human islets were seeded on non-adherent polystyrene plates, PEOT-PBT, and poly(ester urethane) microwell scaffolds coated with ECM proteins. Several controls were prepared by seeding islets on ultra-low adhesive plates and BSA coated tissue culture plates, non-coated and BSA coated microwell scaffolds made from both individual polymers. 


\section{Immunofluorescence staining}

The morphology of islets was evaluated by histological analysis and microscopy. Following fixation in $4 \%(\mathrm{w} / \mathrm{v})$ paraformaldehyde and permeabilization by $0.3 \%$ Triton for 3 hours, human islets were immunofluorescent labeled for glucagon and insulin [16]. The islets were blocked with 10\% normal goat serum/0.15\% Triton-X $100 / 10 \mathrm{mM}$ PBS overnight at $4^{\circ} \mathrm{C}$. The primary and secondary antibodies were diluted in $1 \%$ BSA $/ 0.2 \%$ Triton X-100/10 mM PBS. Staining for glucagon was performed using primary rabbit anti-glucagon (1:100, Abcam, The Netherlands), and two different secondary antibodies including biotin anti-rabbit (1:200, Abcam, The Netherlands) and streptavidin-AlexaFluor 488 (1:200, Abcam, The Netherlands) to distinguish alpha from $\beta$-cell . In case of insulin positive $\beta$-cells, guinea pig anti-insulin (1:200, Abcam, The Netherlands) was used as the primary antibody while AlexaFluor-647 goat anti-guinea pig (1:500, Abcam, The Netherlands) was applied as the secondary conjugated antibody. Nuclei were stained using with 4',6'-diamidino-2-phenylindole dihydrochloride DAPI (Abcam, The Netherlands) for $10 \mathrm{~min}$. Each step was followed by subsequent washing in $1 \%$ BSA/ $0.2 \%$ Triton X-100 in $10 \mathrm{mM}$ PBS. Samples were subjected to optical sectioning in axial (z) dimension using a Zeiss confocal laser-scanning microscope.

\section{Glucose stimulated insulin secretion tests}

Glucose-stimulated insulin secretion (GSIS) tests were performed using triplicate samples of 50 human islets for each condition at 3 and 7 days after seeding. Each sample was first incubated for 1 hour in a glucose free incubation buffer, in a modified Krebs-Ringer bicarbonate buffer with HEPES (KRBH) containing 115 $\mathrm{mmol} / \mathrm{L} \mathrm{NaCl}, 5 \mathrm{mmol} / \mathrm{L} \mathrm{KCL}, 24 \mathrm{mmol} / \mathrm{L} \mathrm{NaHCO}_{3}, 2.2 \mathrm{mmol} / \mathrm{L} \mathrm{CaCl}_{2}, 1 \mathrm{mmol} / \mathrm{L}$ $\mathrm{MgCl}_{2}, 20 \mathrm{mmol} / \mathrm{L}$ HEPES, and $2 \mathrm{~g} / \mathrm{L}$ human serum albumin, $\mathrm{pH}$ 7.4. Samples were then exposed to low glucose $(1.6 \mathrm{mmol} / \mathrm{L})$, high glucose $(1.6 \mathrm{mmol} / \mathrm{L})$, and another 
low glucose (1.6 mmol/L) $\mathrm{KRBH}$ buffer each for $90 \mathrm{~min}$ at $37^{\circ} \mathrm{C}$. After each incubation step, samples' mediums were collected for insulin concentration measurements by ELISA (Mercodia, Uppsala, Sweden). Finally, the islets from each sample were collected for DNA quantification using a Quant-iT picogreen dsDNA assay (Invitrogen).

\section{Statistical analysis}

Results are presented as the mean \pm standard deviation. Statistical analysis was performed using Student's T-test, One-way ANOVA and the least-significant difference (LSD) multiple comparison test by application of SPSS statistic software (Chicago, USA). Statistical significance was considered at a p-value $\leq$ 0.05 .

\section{Results}

\section{Microwell scaffold morphology}

The poly(ester urethane) and PEOT-PBT microwell scaffolds were fabricated using micro thermoforming (Figure 1A). SEM analysis indicated identically shaped microwells distributed homogenously across the surface (Figure 1). The process was highly accurate and reproducible and all microwell scaffolds remained form stable during handling. PEOT-PBT microwells had a circular and tapered appearance with aperture diameter of $380 \pm 5 \mu \mathrm{m}$ and bottom diameter of $300 \pm 5$ $\mu \mathrm{m}$, and distance between aperture and base of $300 \pm 10 \mu \mathrm{m}$. Poly(ester urethane) microwells had a similar aperture diameter but a different base diameter of $250 \pm 5$ $\mu \mathrm{m}$. 




Film insertion
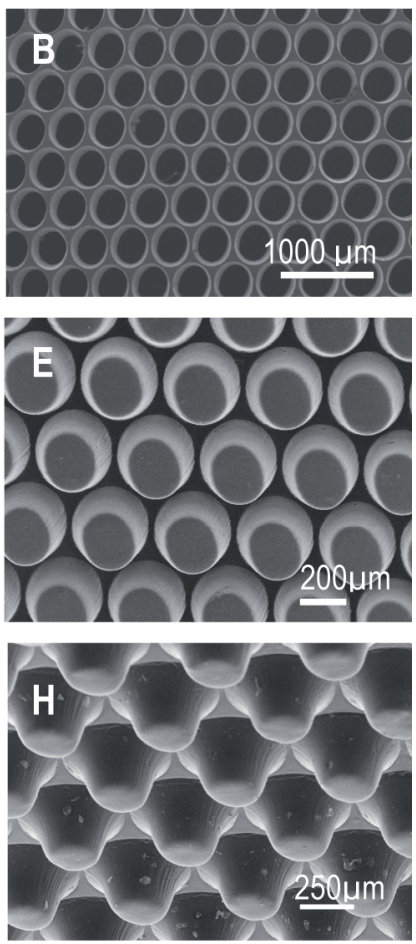


Figure1. The structure of microwell scaffolds. A) Schematic representation of the fabrication of microwell scaffolds using micro-back molding. A1) Press device; A2) Backing material; A3) PEOT-PBT or poly(ester urethane) film; A4) mold A5) Microthermoformed microwell scaffold. SEM images of B-D) mold structure for applying thermoforming on polymer films E, F) PEOT-PBT microwell scaffold G) human islets in PEOT-PBT scaffold $\mathbf{H}$, I) poly(ester urethane) microwell scaffold $\mathbf{J}$ ) human islets in poly(ester urethane) microwell scaffold. 


\section{Function of primary human islets}

To assess the effect of the culture substrate material (i.e. non-treated culture plastic, PEOT-PBT, and poly(ester urethane) and culture substrate topography (i.e. flat films versus microwells) islets functionality was studied. To this end, human islets were cultured for 7 days on the different culture substrates and topographies and subjected to a glucose stimulation insulin secretion test at day 3 and 7 . Islets cultured in the PEOT-PBT and poly(ester urethane) microwell scaffolds released significantly more insulin than the control islets that were cultured on ultra-low adhesive plastic plates (Figure 3). Moreover, the increase in insulin secretion showed a consistent yet discrete tendency to be highest in islets cultured on PEOTPBT groups. In addition, islets seeded in microwells secreted more insulin than islets seeded on flat membranes of the same materials. Furthermore, while islets on a flat surface showed a consistent decline in insulin release over time, islets cultured in microwells increased their insulin secretion over time.

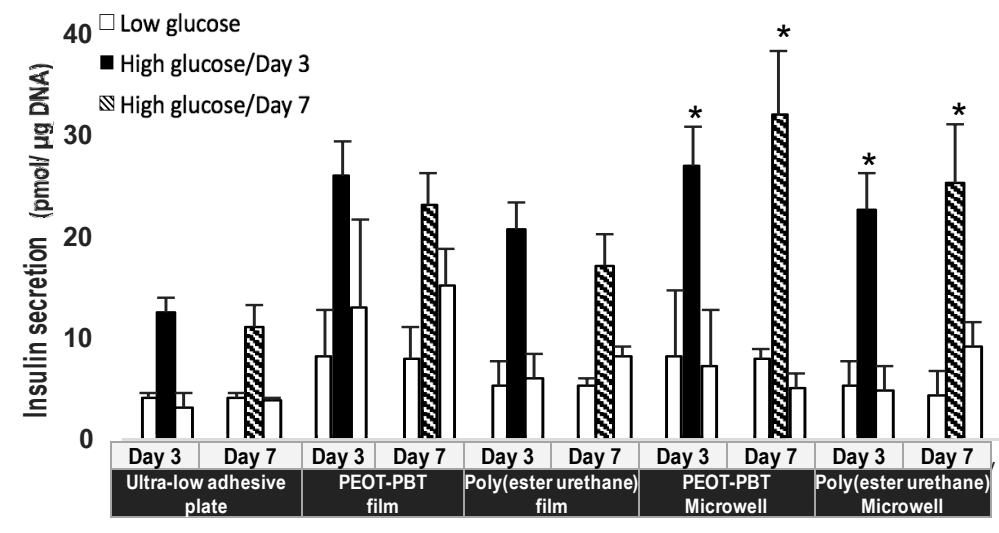

Figure 2. Glucose stimulated insulin secretion test of human islets on ultra-low adhesive plate (control), PEOT-PBT and poly(ester urethane) polymer film and microwell scaffold at day 3 and $7 . *=p<0.05$. 


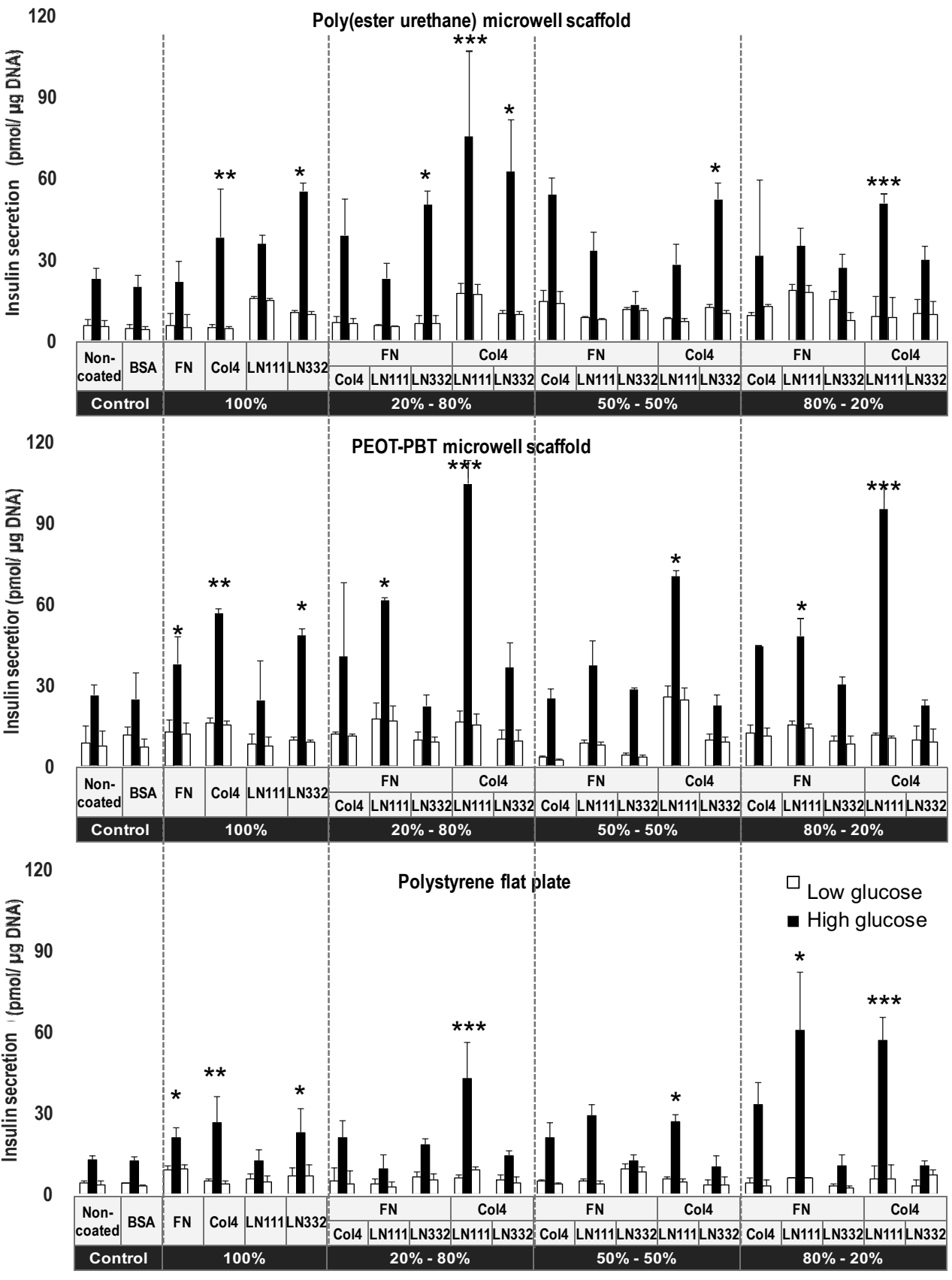

Figure 3. Glucose stimulated insulin secretion test of human islets on monolayer coated ECM proteins on poly(ester urethane) microwell films (top panel), PEOT-PBT microwell films (middle panel) and polystyrene tissue culture plastic (bottom panel) with different ratios of ECM proteins at day $3 .{ }^{*}, * *$, and $* * * \mathrm{p}<0.05,<$ 


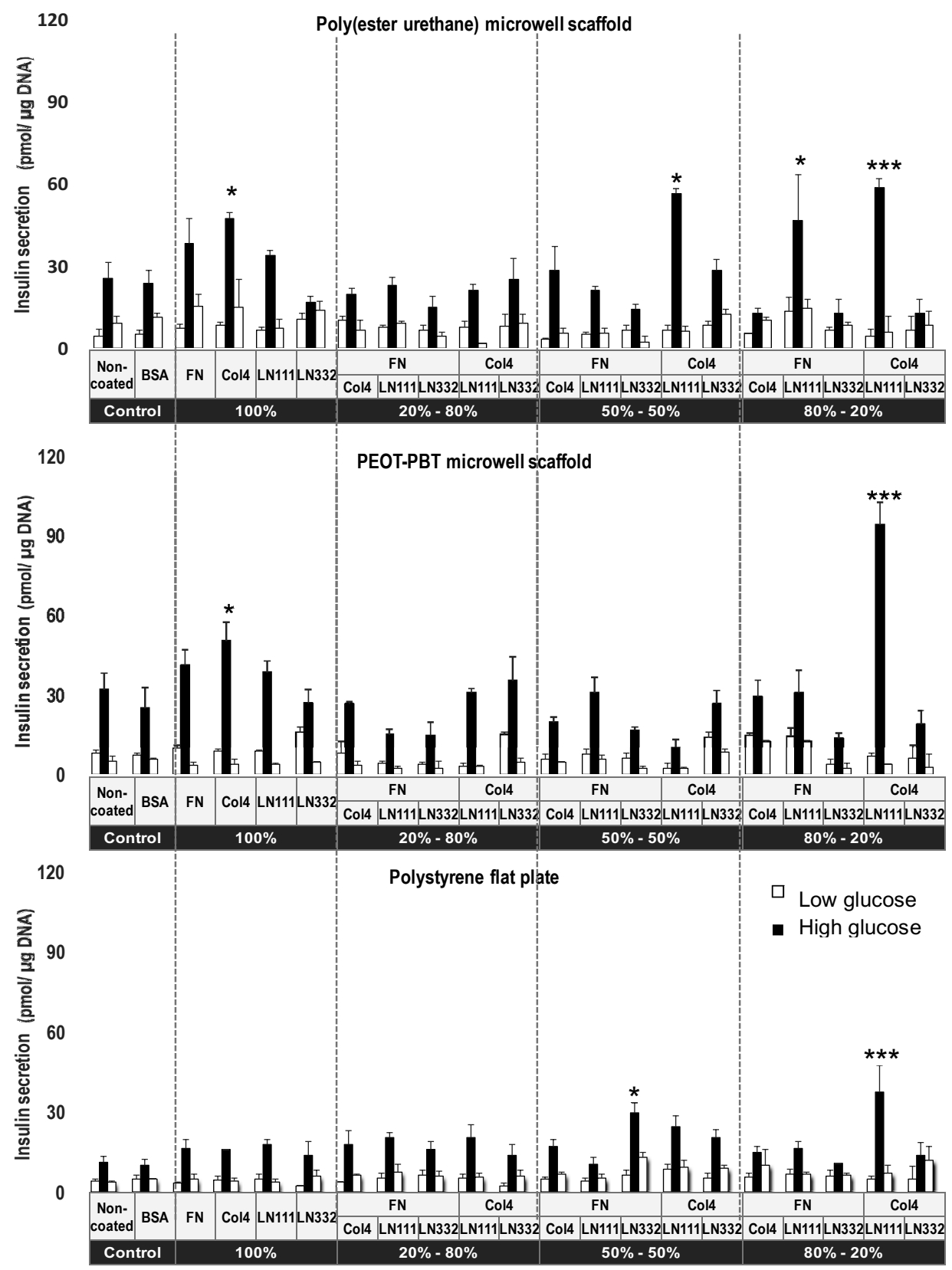

Figure 4. Glucose stimulated insulin secretion test of human islets on monolayer coated ECM proteins on poly(ester urethane) microwell films (top panel), PEOT-PBT microwell films (middle panel) and polystyrene tissue culture plastic (bottom panel) with different ratios of ECM proteins at day $7 . *{ }^{* *}$, and $* * * \mathrm{p}<0.05,<0.01$, and $<0.001$, respectively. 
The PEOT-PBT and poly(ester urethane) microwell scaffolds were coated with FN, Col4, LN111 or LN332. Additionally, these molecules were pairwise combined at a ratio of 5:5, 2:8 and 8:2. After seeding the human islets on coated microwells, the effect of the different ECM molecules on insulin secretion at day 3 and day 7 were assessed and values were normalized to the amount of insulin secreted per cell. The results were compared to control groups consisting of human islets cultured on non-coated and BSA coated PEOT-PBT and poly(ester urethane) microwells. Islets on flat polystyrene plates significantly increased their glucose secretion level in the presence of FN, Col4, LN 332, Col4:LN111 (5:5, 2:8 and 8:2), and FN: LN111 (8:2) at day 3 (Figure 3). Similar results were observed for the islets seeded on the ECM coated microwell scaffolds. We observed that the amount of insulin secreted by islets seeded on ECM molecule coated PEOT-PBT scaffolds was higher than on poly(ester urethane) scaffolds, and significantly higher compared to islets seeded on polystyrene (Figure 4). We observed that insulin secretion in the microwell scaffold groups (Figure 3 and 4) coated with both FN and Col4 show a significantly increased insulin secretion compared to the control group on both day 3 and 7 of culture. The insulin secretion from islets seeded on PEOT-PBT and poly(ester urethane) microwells scaffolds, and tissue culture polystyrene coated with LN111 was lower than the control group on day 3 . However, insulin secretion increased in islets cultured in microwell scaffolds after seven days, while in the tissue culture polystyrene group no change was observed. Islets cultured on LN332 coated scaffolds show an enhanced insulin secretion only after 3 days but not 7 days. Comparison of insulin secretion of islets cultured on Col4 at day 7 indicate a significant better glucose response in the two microwell scaffold groups, but not in the polystyrene group.

Interestingly, a significant increase in insulin secretion was observed for Col4 and LN111 (ratios used 5:5 and 8:2) coated scaffolds at day 3. Although at seven days a slight decrease in insulin secretion was observed in most conditions, islets cultured on Col4:LN111 (8:2) coated microwell scaffolds showed a clear increase of insulin secretion (Figure 3 and 4). The outcome from islets cultured on 
Col4:LN111 coated scaffolds show consistently that the $\beta$-cell's endocrine function is significantly improved in the presence of these specific ECM proteins.

By comparing the islet function (stimulation indices figure S1) in non-coated microwell scaffolds with tissue culture polystyrene the positive influence of the 3D microwell shape on the islet's endocrine function can be analyzed. In both poly(ester urethane) and PEOT-PBT microwell scaffolds glucose responsiveness of islets increased between day 3 and 7, while on tissue culture polystyrene a slight decrease of insulin release was seen. In short, islets on flat polystyrene plate as well as microwell groups coated with FN, Col4, LN332 and Col4:LN111 (5:5, 2:8 and 8:2) and FN:LN111 (8:2), FN:Col4 (8:2) indicated significantly increase of glucose secretion at day 3 . Following the insulin release, revealed that the insulin release decreased in the presence of aforementioned ECM molecules overtime (from day 3 to 7). However, stimulation index for the flat polystyrene and PEOT-PBT and poly(ester urethane) microwells coated with a combination of Col4:LN111 (8:2) was significantly higher than non-coated groups at both day 3 and 7. It indicated the importance of this combination of Col4:LN111 (8:2) for the function of islets over a longer time span.

\section{Islet structure on the ECM coated surfaces}

To assess whether the presence of ECM molecules on biomaterials can affect islet morphology, human islets were seeded on flat surface polystyrene plates coated with ECM molecules. The ECM proteins include FN, Col4, LN111, LN332, a mixture of LN111, 211, 121, 311, 411 and 511, as well as pairwise combinations of FN, Col4 and LNs at ratios of 5:5, 2:8 and 8:2. Additionally, ultra-low adhesive plates were used as negative controls. Islets were stained for insulin and glucagon using immunofluorescent labeling and imaged using phase contrast transmission light, and confocal fluorescent microscopy. 
We observed that the islets in the control group did not adhere to the surface of non-treated plates and that they kept their round shape until day 3. Histological imaging revealed that in the core of these islets functional $\beta$-cells were mainly lost (Figure 5) [17, 18].

The islets on FN and Col4 coated substrates adhered to the surface and spread over a 7 days period, which resulted in loss of their round morphology. Islets cultured on LN332 coated substrates had partially lost their round morphology. The islets cultured on substrates with coatings of Col4 and LN111 at 5:5 and 8:2 ratios, showed partial cell adherence to the underlying substrate fixing the islets in place, while their round morphology was preserved. Moreover, histology revealed higher numbers of functional $\beta$-cells after 7 days of culture for the islets cultured on substrates coated with of Col4 and LN111 at 5:5 and 8:2 ratios (Figure 5).

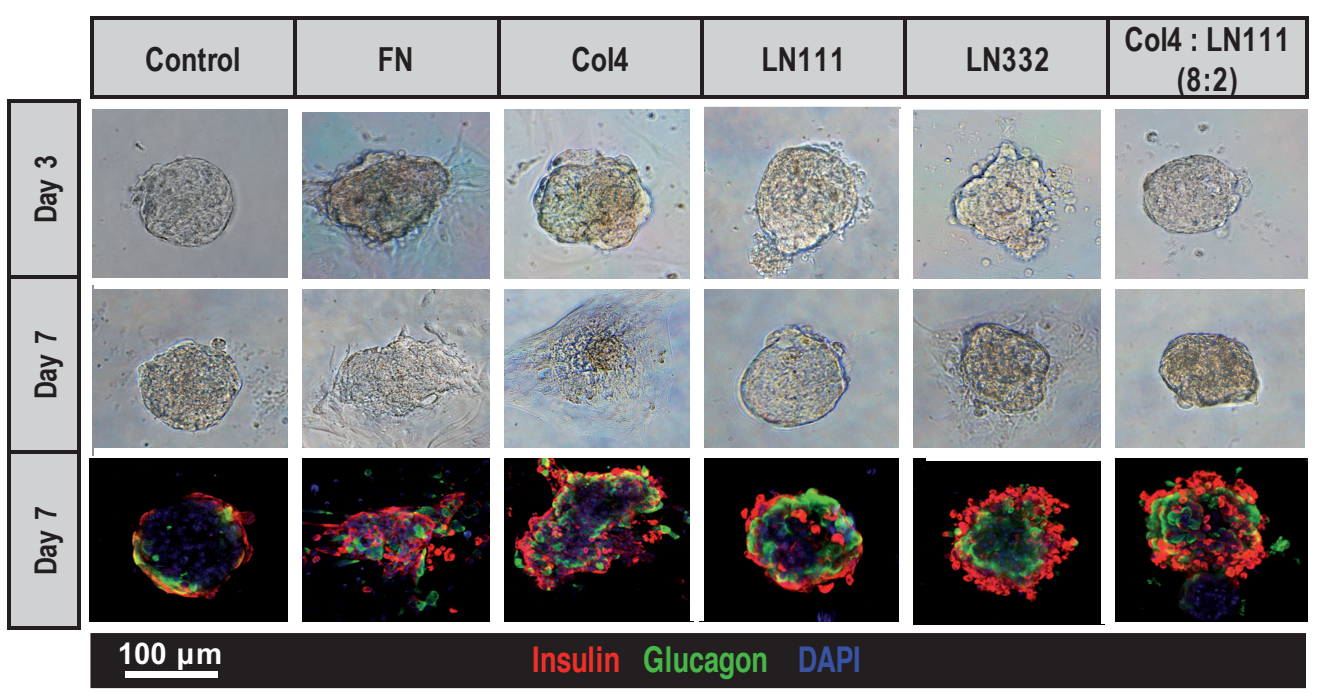

Figure 5. Morphology of islets on polystyrene coated with FN, Col4, LN111, LN332 and a combination of Col4 and LN111 at a ratio of 8:2 at day 3 and 7. The islets cultured on ultralow adhesive plates was used as a control group. 


\section{Discussion}

Islet transplantation is an efficient treatment for type one diabetes. However, it faces with many obstacles such as reduced function of islets or loss of $60 \%$ of the islets in a short period of time after transplantation [19, 20]. For clinical islet transplantation, the islet extracellular matrix is degraded by the enzymes used during the isolation procedure. It was also reported that the $\beta$-cells do not form the extracellular matrix but the matrix is provided by local capillary endothelial cells that produce the basement membrane (BM) [21]. Therefore, it is critical to support transplanted islets with external ECM molecules, particularly in the first 2 weeks, until revascularization and deposition of new ECM can take place [22]. The aim of this study was to investigate the effect of specific islet ECM molecules on function and cell behavior. The selection of ECM molecules was based on their presence in the microenvironment and their influence on the physiology of islets. It is known that the signaling interaction between the ECM molecules and islet cells can regulate multiple aspects of islets physiology such as insulin release, survival and proliferation of $\beta$-cells [23]. Considering that for successful islet transplantation, it is necessary to promote insulin secretion and improve survival of islets, we select FN, Col4, LN111 and LN332 that have shown to boost the function and viability of islets $[3,24,25]$. In this study not only we assessed cells response to the individual ECM molecules, but also we investigated the combinatorial effect of the ECM molecules on islets to find the best combination to improve the function and viability of the cells. Furthermore, the efficiency of our designed poly(ester urethane) and PEOT-PBT microwell scaffolds was compared to polystryrene flat plate after coating with ECM molecules.

One of the drawbacks of macroencapsulated islets and intrahepatic transplanted islets is the fusion and adhesion of islets. Fusion negatively affects the islet structure and function due to lack of oxygen and nutrients as well as necrosis of cells in the 
core of the islets $[19,26,27]$. We could surpass this limitation by seeding islets in microwells, which resulted in preservation of the islets round shape and prevention of islets fusion. As a consequence of maintaining native like shape of islets, the insulin secretion of islets in the microwells were higher in comparison to islets in flat plate. This change in islets function and morphology might relate to the design of scaffolds. Semi-spherical structure of islets can support them to preserve the islet's round shape. In addition, each microwell could act as a mechanical barrier to avoid fusion of neighboring islets. Thus, the scaffold's design might have had positive influence on islets behavior. Another fact that might have positive influence on islets is related to the material properties of the used substrates. Scaffolds were made of a poly(ester urethane) and PEOT-PBT that are more elastic than polystryrene flat plate. In a study of Seewaldt et al. about the influence of ECM stiffness on breast cancer cells indicated that matrix elasticity can induce physical changes in the tissue that can influence the behavior of tumor cells [28]. To the best of our knowledge, there is no study on the effect of ECM matrix stiffness and elasticity, which might regulate the physiology of the islet cells, thus this field requires more investigation.

Regarding the influence of ECM molecules on islets, the GSIS test indicated significant increase of insulin secretion for the islets that were seeded on substrates coated with either FN or Col4. The positive impact of Col4 on the function of islets in in-vivo [29] and in-vitro [30] was reported previously. Moreover, it was reported that cultivation of islets in the presence of FN increases insulin release and preserves the viability and mass of islets after transplantation [24]. Interestingly, although FN and Col4 molecules individually display a positive effect on islets, base on our findings the combinations of these two molecules have a negative effect on insulin secretion. In favor of this finding, Flaim et al. reported similar results regarding the function of hepatocyte cells [31]. They assessed the effect of Col4 and FN on cellular differentiation of primary rat hepatocytes by immunofluorescent staining of intracellular albumin (a marker of specific liver function). Assessing the interaction of islets with Col and FN indicated strong adhesion of islets to these 
molecules, which resulted in a transition from spheroidal aggregates to monolayers [29]. This will affect the function of the islets and reduce the insulin secretion over time. It is reported that the disruption of the spheroidal architecture of the islets may interfere with integrin-mediated signaling and paracrine interactions between islet cells [32]. The integrin-mediated signaling in response to the pancreatic ECM plays crucial roles in $\beta$-cell survival and insulin secretion. For instance, collagens interaction with integrins including $a 1 \beta 1, a 2 \beta 1, a 3 \beta 1$ regulate islet cell adhesion, insulin gene expression and islet survival [107].

The study of Korpos et al. indicated that LNs of Peri-islet BM (consisting of LN a $2, a 4, \beta 1, \beta 2, \gamma 1, \gamma 2$, and $\gamma 3$ chains) are distinct from the BM of surrounding blood vessels (consisting LN a 5 chains, $\beta 1, \gamma 1$ ) [33]. Since, LN $\beta 1, \gamma 1$ chains are common between Peri-islet BM as well as the BM of surrounding blood vessels, we expected to see significant influence of LN111 on islets. Interestingly, interaction of LN111 with islets did not positively affected insulin secretion. Whereas, LN111 in combination with Col4 showed a significant increase in the insulin secretion even higher than only with Col4. It is reported that Col4 tend to bind to via the intermediary glycoproteins nidogen, or entactin, or via direct interaction to heparin sulfate proteoglycans [34]. This binding seems to play a synergistic role on enhancement of insulin secretion. Comparing all different combinations, the highest level of insulin was seen for Col4:LN111 at a ratio of 8:2 with a concentration of $>5 \mu \mathrm{g} / \mathrm{cm}^{2}$ after 7 days. In addition, our results demonstrated that seeded islets in both microwell and flat substrates coated with Col4:LN111 $(8: 2)$ could preserve their round shape and thus improve the function of islets even further. In addition, the semi-spherical structure of the coated microwells gives the opportunity to surround islets all around with ECM molecules. Whereas, in the flat surface the islet are exposed to ECM molecules only in small part. Therefore, based on our data we postulate that the combination of two ECM molecules (Col4 and LN111 with 8:2 ratio) along with specific design of microwell scaffolds could have a positive effect on the function of transplanted islets. 


\section{Conclusions}

In this study, we indicated that Cell-ECM interactions are important for function and survival of islets. Understanding the role of each ECM molecule on insulin secretion, viability and morphology of the islets is a key factor to engineer biomimetic microenvironments for a successful islet transplantation to treat type 1 diabetes.

We showed that LN111 ( $>5 \mu \mathrm{g} / \mathrm{ml})$ in combination with other ECM proteins $(<5$ $\mu \mathrm{g} / \mathrm{ml}$ ), results in a decline in insulin secretion over time. In contrast, lower ratios of LN $(<5 \mu \mathrm{g} / \mathrm{ml})$ in combination with other ECM molecules lead to a sustained release of insulin over a 7 days period. Both PEOT-PBT and poly(ester urethane) microwell scaffolds coated with the combination of Col4 and LN111 with a ratio of $8: 2$ could modify the microenvironment of the transplanted islets. Consequently, islets function and viability were enhanced and their spherical structure was supported. The results indicated the importance of choosing a specific combination of ECM molecules at a distinct ratio for successful scaffold functionalization. Based on this study we propose that functionalized microwell scaffolds with Col4:LN111 $(8: 2)$ are the best candidate for islet transplantation due to its positive influence on insulin release and survival of islets. 


\section{Supplemental material}

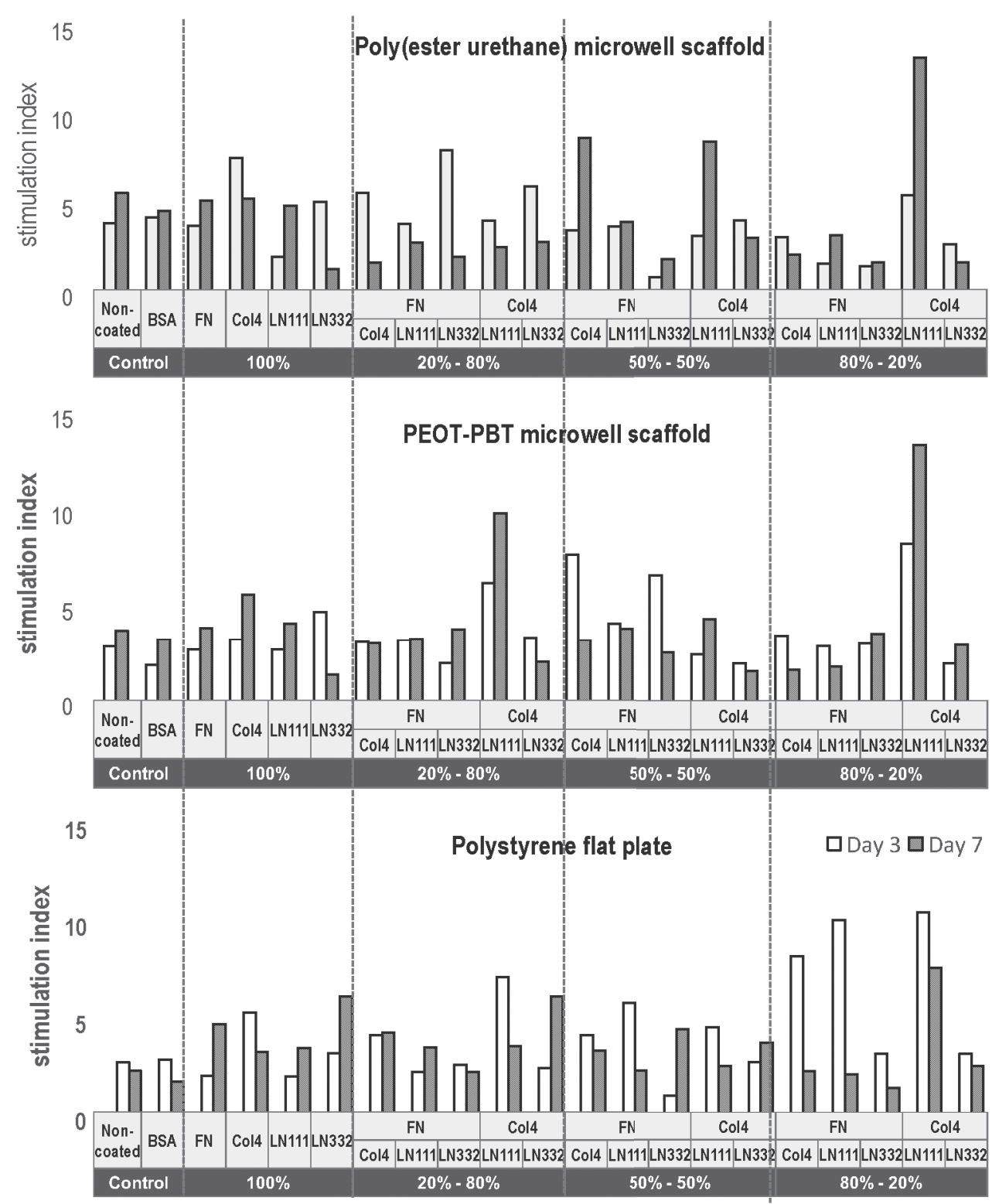

Figure S1. Stimulation index of human islets cultured on monolayer coated ECM molecules on poly(ester urethane) microwell films (top panel), PEOT-PBT microwell films (middle panel) and flat polystyrene tissue culture plastic (bottom panel) with different ratios of ECM proteins at day 3 and 7. 
60

Polystyrene flat plate / Day 7
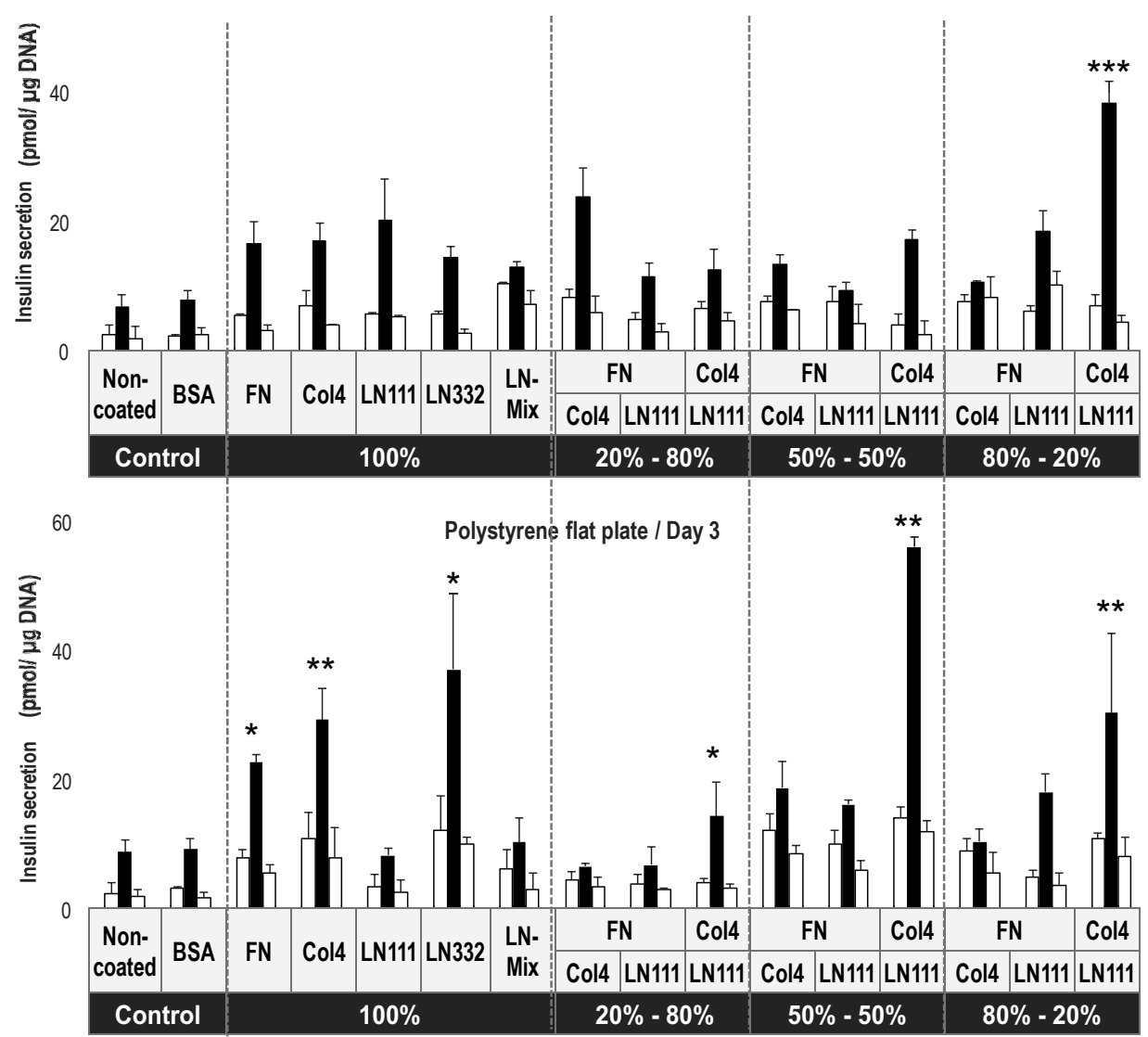

Figure S2. Glucose stimulated insulin secretion test of human islets on ultra-low adhesive plate (Non-coated) and BSA coated plate as controls and monolayer coated ECM molecules on Polystyrene flat plate coated with different ratios of ECM proteins at day 7 (upper panel) and day 3 (bottom panel). 


\section{References}

1. Barton, F.B. et al. (2012) Improvement in outcomes of clinical islet transplantation: 1999-2010. Diabetes care 35 (7), 1436-1445.

2. Davis, N.E. et al. (2012) Enhanced function of pancreatic islets co-encapsulated with ECM proteins and mesenchymal stromal cells in a silk hydrogel. Biomaterials 33 (28), 6691-6697.

3. Kaido, T. et al. (2004) Regulation of human $\beta$-cell adhesion, motility, and insulin secretion by collagen IV and its receptor a1 $\beta 1$. Journal of Biological Chemistry 279 (51), 53762-53769.

4. Beattie, G.M. et al. (1996) Regulation of proliferation and differentiation of human fetal pancreatic islet cells by extracellular matrix, hepatocyte growth factor, and cell-cell contact. Diabetes 45 (9), 1223-1228.

5. Lin, H.-Y. et al. (2010) Fibronectin and laminin promote differentiation of human mesenchymal stem cells into insulin producing cells through activating Akt and ERK. Journal of biomedical science 17 (1), 56.

6. Yap, W.T. et al. (2013) Collagen IV-modified scaffolds improve islet survival and function and reduce time to euglycemia. Tissue Engineering Part A 19 (21-22), 2361-2372.

7. Pinkse, G.G. et al. (2006) Integrin signaling via RGD peptides and anti- $\beta 1$ antibodies confers resistance to apoptosis in islets of Langerhans. Diabetes 55 (2), 312-317.

8. Hammar, E. et al. (2004) Extracellular matrix protects pancreatic $\beta$-cells against apoptosis. Diabetes 53 (8), 2034-2041.

9. Lucas-Clerc, C. et al. (1993) Long-term culture of human pancreatic islets in an extracellular matrix: morphological and metabolic effects. Molecular and cellular endocrinology 94 (1), 9-20.

10. Nagata, N. et al. (2001) Evaluation of insulin secretion of isolated rat islets cultured in extracellular matrix. Cell transplantation 10 (4-1), 447-451.

11. Nagata, N. et al. (2002) Co-culture of extracellular matrix suppresses the cell death of rat pancreatic islets. Journal of Biomaterials Science, Polymer Edition 13 (5), 579-590.

12. Hammar, E.B. et al. (2005) Activation of NF- $\kappa B$ by extracellular matrix is involved in spreading and glucose-stimulated insulin secretion of pancreatic beta cells. Journal of Biological Chemistry 280 (34), 30630-30637.

13. Llacua, A. et al. (2016) Extracellular matrix components supporting human islet function in alginate-based immunoprotective microcapsules for treatment of diabetes. Journal of Biomedical Materials Research Part A 104 (7), 1788-1796. 
14. Buitinga, M. et al. (2013) Microwell scaffolds for the extrahepatic transplantation of islets of Langerhans. PloS one 8 (5), e64772.

15. Truckenmüller, R. et al. (2011) Thermoforming of film-based biomedical microdevices. Advanced materials 23 (11), 1311-1329.

16. Buitinga, M. et al. (2016) Coculturing human islets with proangiogenic support cells to improve islet revascularization at the subcutaneous transplantation site. Tissue Engineering Part A 22 (3-4), 375-385.

17. Komatsu, H. et al. (2017) Oxygen environment and islet size are the primary limiting factors of isolated pancreatic islet survival. PloS one 12 (8), e0183780.

18. Giuliani, M. et al. (2005) Central necrosis in isolated hypoxic human pancreatic islets: evidence for postisolation ischemia. Cell transplantation 14 (1), 67-76.

19. Lehmann, R. et al. (2007) Superiority of small islets in human islet transplantation. Diabetes 56 (3), 594-603.

20. Ricordi, C. et al. (1990) Islet isolation assessment in man and large animals. Acta Diabetologica 27 (3), 185-195.

21. Nikolova, G. et al. (2006) The vascular basement membrane: a niche for insulin gene expression and $\beta$ cell proliferation. Developmental cell 10 (3), 397-405.

22. Menger, M.D. et al. (1989) Angiogenesis and hemodynamics of microvasculature of transplanted islets of Langerhans. Diabetes 38 (Supplement 1), 199-201.

23. Stendahl, J.C. et al. (2009) Extracellular matrix in pancreatic islets: relevance to scaffold design and transplantation. Cell transplantation 18 (1), 1-12.

24. Hamamoto, Y. et al. (2003) Beneficial effect of pretreatment of islets with fibronectin on glucose tolerance after islet transplantation. Hormone and metabolic research 35 (08), 460-465.

25. Jiang, F.-X. et al. (2002) Distinct distribution of laminin and its integrin receptors in the pancreas. Journal of Histochemistry \& Cytochemistry 50 (12), 1625-1632.

26. Nittala, A. et al. (2007) Investigating the role of islet cytoarchitecture in its oscillation using a new $\beta$-cell cluster model. PLoS One 2 (10), e983.

27. MacGregor, R. et al. (2006) Small rat islets are superior to large islets in in vitro function and in transplantation outcomes. American Journal of PhysiologyEndocrinology and Metabolism 290 (5), E771-E779.

28. Seewaldt, V. (2014) ECM stiffness paves the way for tumor cells. Nature medicine 20 (4), 332-333.

29. Kaido, T. et al. (2006) Impact of defined matrix interactions on insulin production by cultured human $\beta$-cells. Diabetes 55 (10), 2723-2729. 
30. Blomeier, H. et al. (2006) Polymer scaffolds as synthetic microenvironments for extrahepatic islet transplantation. Transplantation 82 (4), 452.

31. Flaim, C.J. et al. (2005) An extracellular matrix microarray for probing cellular differentiation. Nature methods 2 (2), 119-125.

32. Cabrera, O. et al. (2006) The unique cytoarchitecture of human pancreatic islets has implications for islet cell function. Proceedings of the National Academy of Sciences of the United States of America 103 (7), 2334-2339.

33. Korpos, É. et al. (2013) The peri-islet basement membrane, a barrier to infiltrating leukocytes in type 1 diabetes in mouse and human. Diabetes 62 (2), 531-542.

34. Olsen, B. (1993) Basement membrane collagens (type IV). Guidebook to the Extracellular Matrix and Adhesion Proteins. 



\title{
Chapter 6
}

PEOT-PBT and poly(ester

urethane) microwell scaffolds for extrahepatic islet transplantation

\author{
E. Hadavi \\ A.M. Smink \\ P. de Vos \\ B.J. de Haan \\ R. de Vries \\ J. Leijten \\ A.A. van Apeldoorn \\ H.B.J. Karperien
}




\section{Abstract}

Intrahepatic islet transplantation is associated with significant loss of islet mass, which hinders long term insulin independence. Extrahepatic islet transplantation by means of a macro-encapsulation method has the potential to improve the survival and function of transplanted islets. Macro-encapsulation has numerous advantages including that it can contain a large number of islets, provides mechanical support and protection, and can be easily retrieved when necessary. However, the selection of a suitable biocompatible material, ability to prevent islet aggregation and fusion, capacity to allow angiogenesis to surround and permeate the implant, and the choice of implantation site have remained challenges that have mitigated this approach's potential.

In the present study, PEOT-PBT and poly(ester urethane) microwell scaffolds were developed to provide a platform for extrahepatic islet transplantation, which prohibits islet fusion. In addition, the scaffolds were fabricated with defined macro-sized pores to stimulate islets revascularization. Both polymers are biodegradable and have similar mechanical properties, but differed in their respective degradation time. The microwell scaffolds were implanted in the intraperitoneal and subcutaneous site of Albino Oxford (AO) rats to test the biocompatibility of the scaffolds. Histological analysis indicated that both type of scaffolds were biocompatible. The structure of PEOT-PBT microwell scaffolds was stable for 12 weeks, while the poly(ester urethane) was fragmented 12 weeks post-implantation. Isolated islets from AO rats were in the microwell scaffolds and transplanted intraperitoneally in Rowett nude rats to study the in vivo survival and function of islets in our scaffolds. Our finding demonstrated rapid islet mass loss shortly after transplantation. Histological analysis indicated that insufficient vascularization could be the main reason of the reduction in islets number and function. Comparing both scaffolds, we demonstrated that PEOT-PBT is suitable for islet transplantation based on its biocompatibility, structural stability, and capability to preserve islets structure. 


\section{Introduction}

Type one diabetes is an autoimmune disease in which the immune reaction of body destroys the insulin producing $\beta$-cells in pancreatic islets. The total number of people in the world with diabetes is predicted to rise from 171 million in 2000 to 366 million in 2030, of which type 1 diabetes accounts for between $5 \%$ and $10 \%$ [1]. Type I diabetic patients rely on daily self-administration of exogenous insulin to control their blood glucose level. This method lacks precise glycemic control, which over time may cause severe complications such as neuropathy, nephropathy, retinopathy and can increase the risk of cardiovascular diseases [2].

Intra-hepatic islet transplantation is a promising therapy to treat type 1 diabetes [3] [4]. It is a relatively easy procedure by infusion of donor islets into the portal vein with minimal side effects. However, a rapid decrease of transplanted islet mass, which is caused by multiple factors, limits the wide-spread implementation of this therapy. It is shown that direct injection of the islets leads to an activation of the hemostasis chain reaction and the instant blood mediated inflammatory reaction (IBMIR), which results in islets loss [5]. Moreover, mechanical stress as well as exposure of a lowered oxygen tension due to impaired vascularization will also negatively affect the survival and function of injected islets. Rapid islet loss is further complicated by the limited availability of suitable pancreas donors [6-8].

An alternative method to overcome the aforementioned limitations is to transplant islets to extrahepatic sites. Significant challenges of extrahepatic islet transplantation are islets diffusion and weak vascularization upon implantation. We used a tissue engineering approach, which is an interdisciplinary field involving the use of cells and materials to repair or generate specific tissues or organs, to engineer an efficient islet transplantation device. 
To this end, we have previously developled a PEOT-PBT porous microwell scaffolds [9]. However, the developed platform was missing an immediate vascularization into the scaffolds to facilitate nutrient and hormonal exchange with islets. In addition, the former design had limited capacity to encapsulate islets. As a consequence, the scaffold was applicable to only small animals like mice. To overcome these limitations, we further optimized and up-scaled the previously developed PEOT-PBT microwell scaffolds by increasing the porousity and size of the scaffolds. Presence of more micro pores can facilitate and accelerate vascularization. Also, larger microwells can encapsulate higher number of islets that is required for islet transplantation in human. Furthermore, we developed a new porous microwell scaffold made of a poly(ester urethane). The short degradation time of this polymer could allow islets to be surrounded by the natural microenvironment of the body soon after transplantation. The microwell shape of both PEOT-PBT and poly(ester urethane) scaffolds could inhibit islets fusion. Additionally, both scaffold designs include a lid to prevent islet loss. Prior to evaluating the efficiency of these scaffolds in vivo to investigate whether the changes would indeed overcome the previous limitations, we performed in vitro studies. In vitro studies on our newly developed scaffolds demonstrated improved insulin release and islets survival.

Moreover, we found that the architecture of the scaffold can preserve the morphology of islets and prevent clustering and clumping of them. Both used polymers were biodegradable and have approval for certain human applications. In the current work, we performed an in vivo experiment to evaluate the function and survival of isogenic islets within our scaffolds. The main aim of this study was to investigate and compare the efficiency of both scaffolds, with encapsulated islets after transplantation in a diabetic rat model with a follow up of 5 months. 


\section{Materials and methods}

\section{Experimental design}

In order to investigate biocompatibility and functionality of our novel scaffold, we performed two experiments. In the first experiment, the biocompatibility of the biomaterials, which were used for fabrication of the scaffolds, were investigated. Thus, PEOT-PBT and poly(ester urethane) microwell scaffolds were implanted subcutaneously and intraperitoneally in Albino Oxford (AO) rats. Biocompatibility of each polymer was examined at 1,4 and 12 weeks post-implantation $(n=4)$.

In the second experiment, islet function in PEOT-PBT and poly (ester urethane) microwell scaffolds was investigated in vivo $(n=4)$. To this end, islets were isolated from AO rats and loaded in PEOT-PBT or poly(ester urethane) microwell scaffolds. The cell laden devices were transplanted in intraperitoneal site of streptozotocininduced diabetic Rowett rats. As a control group for the functional assay, islets were transplanted under the kidney capsule of Rowett rats $(n=4)$. To monitor the function of islets, non-fasting blood glucose level was measured every day and oral glucose tolerance test (OGTT) was performed two and four weeks post transplantation. Both control and test groups were transplanted with approximately 3000 islets.

\section{Fabrication of PEOT-PBT films}

The polymer films were fabricated using biodegradable PEOT-PBT copolymers that were composed of soft block of poly(ethylene oxide terephthalate) and hard block of poly(butylene terephthalate) with the composition 4000PEOT30РВT70 (Polyvation BV, Groningen, the Netherlands, under the trade name PolyActive ${ }^{\mathrm{TM}}$ ). The thin film scaffolds with thickness of $40 \mu \mathrm{m}$ were fabricated using solvent casting. $15 \%(\mathrm{w} / \mathrm{w})$ PEOT-PBT was dissolved in 65\% Chloroform (Merk, Darmstadt, Germany) and 35\% (w/w) 1,1,1,3,3,3-hexafluoro-2-isopropanol 
(Biosolve, Valkenswaard, the NPEOT-PBTlands) and the solution was casted on silicon wafer at a height of $400 \mu \mathrm{m}$ at room temperature. The polymer-films were dried under nitrogen stream for four hours following incubation in ethanol to remove residual solvents and scaffolds were peeled off. Subsequently, the polymer films were dried in a vacuum oven (Heraeus, Hanau, Germany) at $30^{\circ} \mathrm{C}$ for three days to complete solvent evaporation. The films were used for fabricate microwells scaffolds and their lids.

\section{Fabrication of poly(ester urethane) films}

The poly(ester urethane) was provided by Polyganics (Groningen, the Netherlands). It was synthesized by reaction of an a, $\omega$-isocyanate end functionalized pre-polyester and an a,w-hydroxy bis-carbamate (Chapter 3). Thin poly(ester urethane) films with a thickness of $40 \mu \mathrm{m}$ were fabricated using solvent casting. The poly(ester urethane) was dissolved in chloroform (Merck, Darmstadt, Germany) at a concentration of $5 \mathrm{wt} \%$ and casted on a silicon wafer at room temperature. The polymer films were dried under a continuous nitrogen stream for four hours followed by immersion in ethanol to remove residual solvents and allowed for facile collection of the scaffold. Finally, the polymer films were dried in a vacuum oven at $30^{\circ} \mathrm{C}$ for 3 days to complete the solvent evaporation.

\section{Laser drilling}

Scaffolds were composed of a membrane containing microwells that acted as islet containers and a flat film that acted as a lid for the microwell array. In both membranes, laser drilling was used for the introduction of small holes as described previously [9]. Laser drilling was performed by Veldlaser Company ('s-Heerenberg, Netherlands). PEOT-PBT and poly(ester urethane) films were drilled in two different sizes for fabrication of microwells and lid of the scaffolds, respectively. PEOT-PBT films for microwell arrays were drilled with a hole size of $18 \mu \mathrm{m}$ using a $50 \mu \mathrm{m}$ pitch. PEOT-PBT films for microwell lids were drilled with a hole size of 38 $\mu \mathrm{m}$ in dimension, a $100 \mu \mathrm{m}$ pitch, and an outer dimension of $3 \mathrm{~mm}$. Poly(ester 
urethane) films for microwell arrays were drilled with a hole size of $15 \mu \mathrm{m}$, a 50 $\mu \mathrm{m}$ pitch, and an outer dimension of $3 \mathrm{~mm}$. Poly(ester urethane) films for microwell lids were drilled with a hole size of $40 \mu \mathrm{m}$, a $100 \mu \mathrm{m}$ pitch, and an outer dimension of $3 \mathrm{~mm}$.

\section{Fabrication of PEOT-PBT and poly(ester urethane) microwell scaffolds}

The scaffolds consisting of 4630 microwells were manufactured by means of micro-back molding. Micro-thermoforming [10] was performed to create a threedimensional microwell structure [9]. Drilled polymer films with a thickness of 40 $\mu \mathrm{m}$ were pressed into the mold backing material. Polyethylene films with a thickness of $560 \mu \mathrm{m}$ were used as mold backing for the PEOT-PBT microwell scaffold. Paraffin films with a thickness of $540 \mu \mathrm{m}$ and polyethylene films with a thickness of $20 \mu \mathrm{m}$ were used for the fabrication of poly(ester urethane) microwell scaffolds. For fabrication of poly(ester urethane) microwell scaffold, the mold pressure was set at $30 \mathrm{kN}$ at $25^{\circ} \mathrm{C}$. When the temperature reached to $30^{\circ} \mathrm{C}, 33^{\circ} \mathrm{C}$, and $41^{\circ} \mathrm{C}$ the pressure was increased to $40 \mathrm{kN}, 50 \mathrm{kN}$, and $60 \mathrm{kN}$, respectively. Immediately at $41^{\circ} \mathrm{C}$, the pressure was released and the sample was laid in ice-cold ethanol for 15 minutes before peeling off the microwell films. Subsequently, the microwells were laid in xylene for $1 \mathrm{~min}$ to remove any traces of paraffin in the microwells. Xylene was removed by rinsing with ethanol for five times followed by rinsing with distilled water. For fabrication of PEOT-PBT microwell scaffolds, the mold pressure was set at $40 \mathrm{kN}$ at $30^{\circ} \mathrm{C}$. The temperature was increased up to $85^{\circ} \mathrm{C}$ while the pressure was kept stable at $40 \mathrm{kN}$. As soon as the temperature reached $85^{\circ} \mathrm{C}$, the pressure was increased to $65 \mathrm{kN}$ and the temperature was set to $20^{\circ} \mathrm{C}$. When the press device was cooled down to $20^{\circ} \mathrm{C}$, the pressure was released completely. Before peeling off the microwells, the microwells were placed in ethanol for $15 \mathrm{~min}$ at room temperature, peeled off, disinfected using 70\% ethanol overnight, washed vigorously with water, and seeded with islets. The porous microwell scaffolds were used for transplantation of the islets. 
For biocompatibility experiments, non-porous microwell scaffolds of non-drilled polymer films were used.

\section{Animals}

Islets were isolated from pathogen free inbred 8 weeks old male Albino Oxford (AO) rats weighing approximately 200 to 250 grams. Pathogen free inbred Rowett nude rats (Hsd: RH-Foxn1 $\mathrm{rnu} / \mathrm{rnu}$ ) weighing 230 to 270 grams with an age of 7 to 10 weeks served as implantation recipients after diabetes induction. The animals were fed with standard raw chow and acidified water ad libitum. The animal experimentation was approved by the Central Authority for Scientific Procedures on Animals (CCD) and the protocols (3006AA) were approved by local animal care committee of Groningen University.

\section{Islet isolation}

Donor islets were isolated from AO rats under isoflurane anaesthesia according to the Serva protocol [11]. In short, after the $\mathrm{V}$ shape abdominal incision, the bile duct was cannulated and perfused using $10 \mathrm{ml}$ syringe consisting of $9.8 \mathrm{ml}$ collagenase NB8 (Serva) dissolved in Hank's balanced salt solution (HBSS; Gibco) supplemented with DNase (Sigma-Aldrich). The pancreas was placed in a $50 \mathrm{ml}$ tube in ice and transported to the water bath. The pancreases were incubated at $37^{\circ} \mathrm{C}$ with $1 \mathrm{mg} / \mathrm{ml}$ collagenase in Krebs-Ringer-Hepes (KRH) containing 10\% BSA in a total volume of $13 \mathrm{~mL}$ for 10 minutes. Then the tissue fragments were allowed to sediment and washed twice with RPMI containing 1\% BSA. Subsequently, the tissue fragments were centrifuged at $1100 \mathrm{rpm}(\sim 220 \mathrm{~g})$ for 75 seconds with brake on and washed twice with RPMI containing 1\% BSA. Next the tissue fragments were incubated at $37^{\circ} \mathrm{C}$ with $0.7 \mathrm{mg} / \mathrm{ml}$ collagenase in $\mathrm{KRH}$ containing $10 \%$ BSA in a total volume of $13 \mathrm{ml}$ for 8 minutes. Afterwards, the tubes were vigorously handshacked for 30 seconds to break up the pancreases in small pieces. The $4^{\circ} \mathrm{C}$ solution of RPMI/NCS was added to the tubes up to $50 \mathrm{ml}$. Subsequently, the isolation procedure was continued on ice. The tissue fragments were centrifuged at $220 \mathrm{~g}$ for 
75 seconds with brake on and washed twice with RPMI/NCS to remove collagenase and leaked exocrine-derived proteases. The suspension was filtered using a $425 \mu \mathrm{m}$ diameter wire mesh to remove the remaining undigested tissue, fat and lymph. Subsequently, the islets were collected and separated from exocrine tissue and quantified by hand picking. The diameter of islets was measured in a $2 \%$ islet suspension using a 25x magnification dissection microscope (Bauch and Lomb BVB125 and 31-33-66) equipped with an ocular micrometer.

\section{Seeding islets in microwell scaffolds}

The microwell scaffolds were placed on sterile concave glass plates. About 3000 islets were counted and then seeded into microwells under a microscope. The presence of islets was confirmed under the microscope. The microwell and lid of the poly(ester urethane) and PEOT-PBT scaffolds were sealed using a soldering pen with a tip ( $2 \mathrm{~mm} \times 0.5 \mathrm{~mm})$. The tip was coated with Teflon to protect from attaching to the polymers in high temperature. After placing the islets inside of the microwell scaffolds, the lid was placed on top of the scaffold and the border of the poly(ester urethane) and PEOT-PBT scaffolds were sealed at $85^{\circ} \mathrm{C}$ and $185^{\circ} \mathrm{C}$ respectively. Scaffolds were either sealed at 6 spots $(n=2$ for the PEOT-PBT and $n=1$ for the poly(ester urethane)) or were fully sealed ( $n=2$ for the PEOT-PBT and $n=3$ for the poly(ester urethane)).

\section{Islet transplantation}

The Rowett nude rats were rendered diabetic via injection of $75 \mathrm{mg} / \mathrm{kg}$ streptozotocin (STZ) (Zonasar, Upjohn Co., Kalamazoo, MI) or $60 \mathrm{mg} / \mathrm{kg}$ streptozotocin IV (Sigma-Aldrich) in the tail vein. Rats with a blood glucose higher than $20 \mathrm{mmol} / \mathrm{L}$, by two constitutive measurements within two weeks, served as islet recipients. The glucose concentration was detected using the Accu-Check Sensor system (Roche, France). The non-fasting blood glucose of the Rowett nude rats was investigated daily following the islets transplantation. 
As a control group, islets were transplanted without scaffolds under the kidney capsule. For this goal, the diabetic Rowett rats received a small incision in the skin and peritoneum above the kidney in order to have access to the kidney capsule. An opening in the kidney capsule was made using a needle, after which a subscapular pocket under the upper pole of the kidney was created using a metal rod. About 3000 islets in the a volume of $10 \mu \mathrm{l}$ were transplanted under the upper pole of the kidney capsule using a flexible capillary tube.

To evaluate islet function in vivo, islets encapsulated in PEOT-PBT or poly(ester urethane) microwell scaffolds were transplanted into the rats. After a midline abdominal incision the macrocapsules including 3000 islets were transplanted in the intraperitoneal site of the diabetic rats. The macrocapsule was partially located under the liver and fixed to the subcutaneous muscle using a small suture to prohibit from moving and folding inside of the intraperitoneal site.

\section{Post-transplantation monitoring}

After transplantation, the non-fasting blood glucose level and the weight of each rat were measured every day. Normoglycaemia was defined as non-fasting blood glucose level under $10 \mathrm{mmol} / \mathrm{L}$. When the blood glucose level was higher than 20 $\mathrm{mmol} / \mathrm{L}$ for a few consecutive days the animal was euthanized. Afterwards the scaffold, kidney and pancreas were removed for histological analysis.

\section{Oral glucose tolerance test (OGTT)}

Glucose tolerance test was performed on the Rowett nude rats in order to determine the islets' function and glucose sensitivity. OGTT test was carried at two and four weeks post transplantation following a fasting period of two hours. For this purpose, animals did not have access to food for 2 hours after which they were fed with 2 grams of normal rat chow (RM1: SDS diets, Essex, United Kingdom), which was dissolved in water for 5 minutes. Rats were trained to eat at least $70 \%$ of the food within 5 minutes. Next, about $100 \mu$ l of blood sample was taken from 
the tail at the following time intervals: 5 minutes before feeding (-5) and 1, 5, 10, $15,20,25,30,45,60,75,90,105$, and 120 minutes after feeding. After centrifugation at $2000 \mathrm{x} \mathrm{g}$ for $10 \mathrm{~min}$ at $4{ }^{\circ} \mathrm{C}$, blood plasma was used to measure the insulin concentration by means of a rat insulin ELISA kit (Mercodia). Manufacture's instruction was followed. Moreover, the insulin: glucose ratio was determined at the time point of the highest insulin peak.

\section{Immunohistochemistry}

To assess the biocompatibility of the implanted microwell scaffolds, the samples containing PEOT-PBT microwell scaffolds and kidney control samples were embedded in paraffin with a HistoStar ${ }^{\mathrm{TM}}$ embedding workstation (ThermoFisher Scientific). Subsequently, $5 \mu \mathrm{m}$ sections were prepared with a microtome (Microm HM 355S, Thermo Scientific). Since the melting point of the poly(ester urethane) is lower than that of paraffin, samples containing the poly(ester urethane) micro well scaffolds were embedded in Tissue Tek (OCT, Sakura, catalogue\# 4583) and sectioned in $5 \mu \mathrm{m}$ sections with a cryotome (Leica, CM3050). All sections were stained with haematoxylin (VWR, VWRK4085-9002) and eosin (Sigma Aldrich, E4009) to evaluate the tissue-biomaterial interactions, or with trichrome (Sigma Aldrich HT15) for the visualization of blood vessels and fibrous tissue. Immunohistochemical analysis was performed to identify the insulin and glucagon producing cells. The morphology of the transplanted islets was also investigated and compared to the healthy islets of the control group. The pancreases from the healthy rats and the islets of the transplanted rats were immersed in $2 \% \mathrm{wt} / \mathrm{v}$ buffered paraformaldehyde in PBS overnight at $4^{\circ} \mathrm{C}$. Then the paraformaldehyde was replaced with PBS and prepared for embedding. Sections were stained overnight at $4^{\circ} \mathrm{C}$ with $2 \mu \mathrm{g} / \mathrm{mL}$ guinea pig anti-insulin (Abcam, ab7842) and $4 \mu$ g/mL mouse monoclonal anti-glucagon (Sigma Aldrich, G2654). Subsequently, samples were incubated overnight at $4^{\circ} \mathrm{C}$ with $5 \mu \mathrm{g} / \mathrm{mL}$ goat anti-guinea pig 
(Thermo Fisher Scientific, A-11075) and $5 \mu \mathrm{g} / \mathrm{mL}$ goat anti-mouse (Fisher Scientific, 1069113) and counterstained for $10 \mathrm{~min}$ with 4,6-diamideino-2phenylindole dihydrochloride (DAPI, Sigma Aldrich, 32670). The stained slides were imaged using a Nikon Eclipse Ti inverted microscope and analysed using ImageJ software (http://rsb.info.nih.gov.ij).

\section{Results}

\section{Scaffolds structure}

Laser drilling effectively endowed the poly(ester urethane) and PEOT-PBT polymers films with arrays of well-defined microholes. The drilled films were fabricated into microwell scaffolds using micro-back molding (Figure 1). SEM analysis indicated homogeneously shaped microwells throughout the scaffold (Figure 1). The process was reproducible and the shape of both types of formatted microwell scaffolds remained stable upon handling. The microells in PEOT-PBT scaffolds preserve their primary structure and shape while deformation of them were observed in poly(ester urethane) scaffolds. In order to the size of each well of PEOT-PBT and poly(ester urethane) microwells, the upper well diameter were $380 \pm 5$ and $425 \pm 5 \mu \mathrm{m}$, the lower well diameter were $290 \pm 5$ and $170 \pm 5 \mu \mathrm{m}$, and the depth of each well was $280 \pm 10$ and $210 \pm 10 \mu \mathrm{m}$, respectively. The wall thickness of the upper and bottom part of the PEOT-PBT wells was $15 \pm 2$ and $26 \pm 3$ $\mu \mathrm{m}$ respectively. The thickness of the upper and bottom part of the poly(ester urethane) wells was $40 \pm 3$ and $15 \pm 4 \mu \mathrm{m}$, respectively. The hole size of the both PEOT-PBT and poly(ester urethane) microwells varied in size depending on their location, which ranged from $15 \mu \mathrm{m}$ at the upper part of the film to $38 \mu \mathrm{m}$ at the bottom of the microwell.

To test the biocompatibility of the scaffolds, thin films of each polymer were placed intraperitoneally as well as subcutaneously in 12 AO rats. Scaffold folding 
was observed at both implantation sites for both polymers. PEOT-PBT and poly(ester urethane) scaffolds did not attach to the subcutaneous tissue up to 12 weeks and the scaffold's structure were stable. Subcutaneous swelling was observed in 3 out of 9 rats in each PEOT-PBT and poly(ester urethane) implanted groups, in which the swelling disappeared between day 7 and day 25. In the intraperitoneal site, most of the scaffolds were covered by vascular-fat tissue from the first week and the thickness of the fat tissue was increasing over time until week 12 . In some rats the scaffolds were displaced from the transplanted site and no swelling or fluid retention was observed (Figure 1).

In order to increase the chance of vascularization inside of the microwells and facilitate mass transport, it was important to prevent the movement and folding of the scaffolds in the transplanted site. Since the scaffolds occupy a large area of the rat's body, some samples in the biocompatible test demonstrated folding of both PEOT-PBT and poly(ester urethane) scaffolds. Thus, to prohibit folding of the scaffolds in the islet transplanted groups, the microwell scaffolds were placed in the intraperitoneal site, partially under the liver and they were fixed to the subcutaneous muscle by a small suture. As a consequence of fixing the scaffolds to the muscle, $50 \%$ of the scaffolds stayed intact and $50 \%$ of them were only partially folded.

Histological assessment of the scaffolds indicated that the structure of the PEOTPBT wells was better preserved than poly(ester urethane) wells. The PEOT-PBT scaffolds transplanted in both intraperitoneal and subcutaneous sites preserved their microwell structure for at least 12 weeks. In contrast, poly(ester urethane) scaffolds showed buckling, increased well interspacing and decreased well depth a week after transplantation. The microwell structure of poly(ester urethane) scaffolds after 1 week in intraperitoneal site were relatively better than subcutaneous site. The wall thickness of poly(ester urethane) scaffolds slightly decreased after 4 weeks and severely lost their shape and were torn apart after 12 weeks (Figure 2). The poly(ester urethane) scaffolds were degraded in the intraperitoneal site, which resulted in the fragmentation and buckling of the well's 
structures after 12 weeks. In contrast, the transplanted poly(ester urethane) scaffolds in the subcutaneous site indicated just minor buckling after 12 weeks (Figure 2).
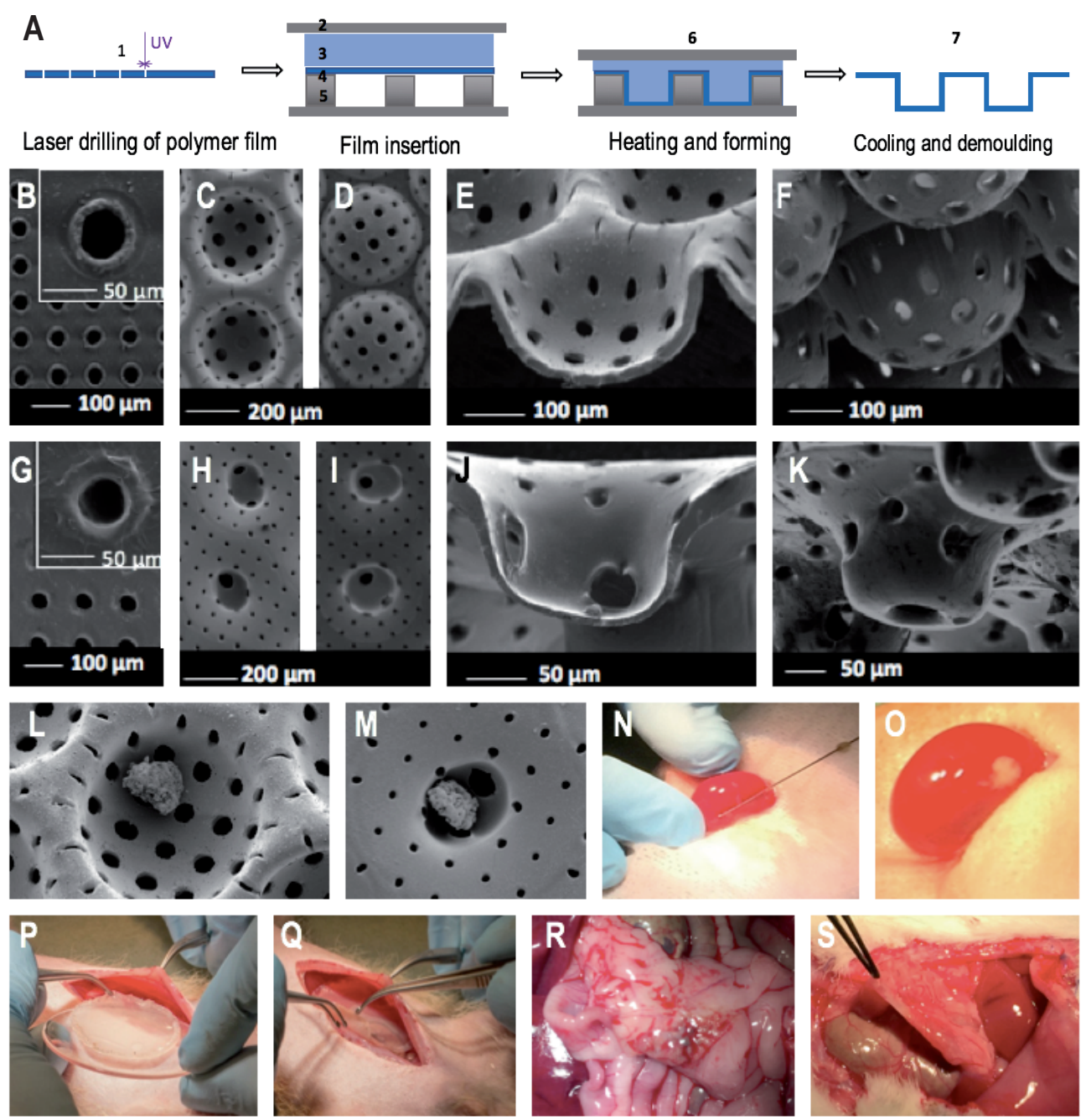

Figure 1. Microwell scaffold structure. A) Schematic representation of the fabrication of microwell scaffold using micro-back molding. A1) Laser drilling of the polymer film, A2) Press device A3) Backing material A4) PEOT-PBT or poly(ester urethane) film A5) mould A6) Microthermoformed microwell scaffold. SEM image of B) Laser drilled PEOT-PBT lid CF) PEOT-PBT microwell scaffold G) Laser drilled Poly(ester urethane) lid H-K) poly(ester urethane) microwell scaffold L) SEM image of seeded islet in PEOT-PBT microwells $\mathbf{M}$ ) SEM image of the seeded islet in poly(ester urethane) microwell, $\mathrm{N}-\mathrm{O}$ ) transplantation of islets 
under the kidney capsule, $\mathbf{P}-\mathbf{Q}$ ) Transplantation of the microwells in intraperitoneal site R) scaffold covered with vascular-fat after 1 week S) scaffold covered with vascular-fat after 12 weeks.

\section{Tissue response against the polymer}

Histological assessment of the samples retrieved in the biocompatibility study indicated that scaffolds did not integrate with tissue and instead they remained as separate layers (Figure 2). Fibrous tissue surrounded the PEOT-PBT implant with a thickness ranging between 50-150 $\mu \mathrm{m}$ at the open side of the scaffold and $14 \pm 4$ $\mu \mathrm{m}$ at the bottom side of the microwells. Even though scaffolds were often folded, the microwells' structure remained intact. Due to omitting of laser drilling in scaffolds of the biocompatibility groups there was a lack of tissue integration with the scaffold, which impeded the biocompatibility analysis. PEOT-PBT scaffolds were evaluated in the sagittal direction on the condition that adipose tissue had grown over the scaffold.
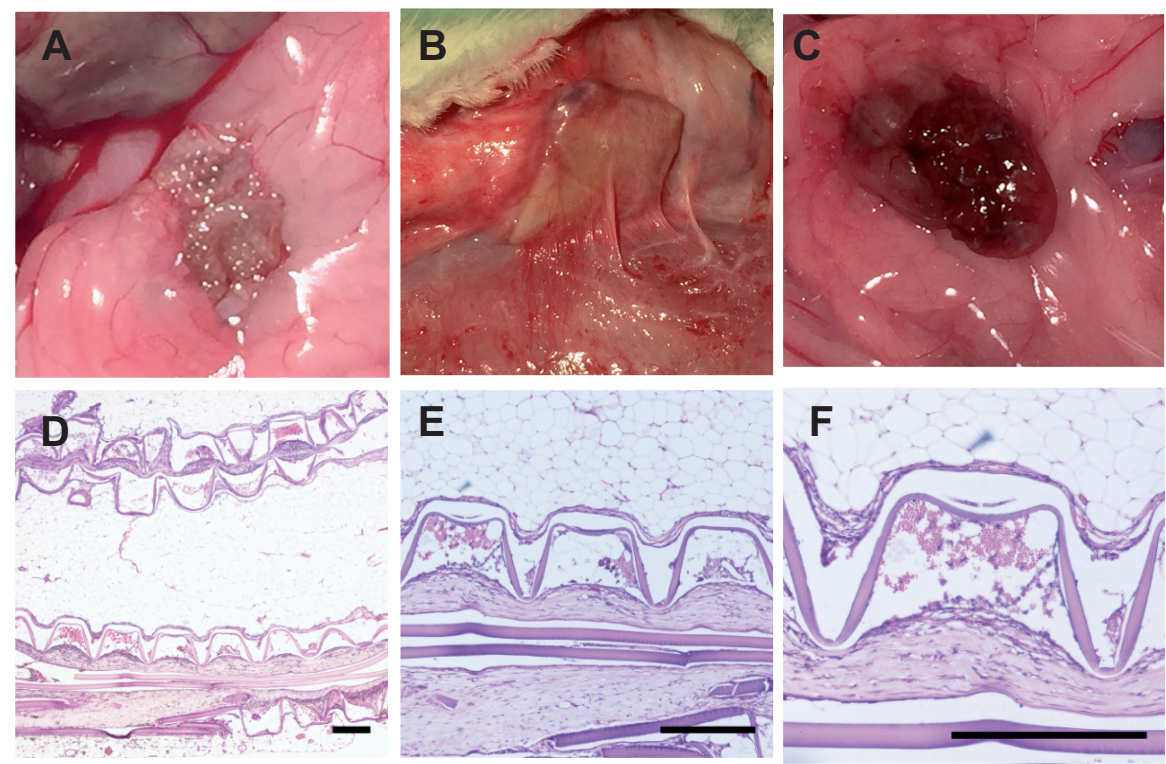

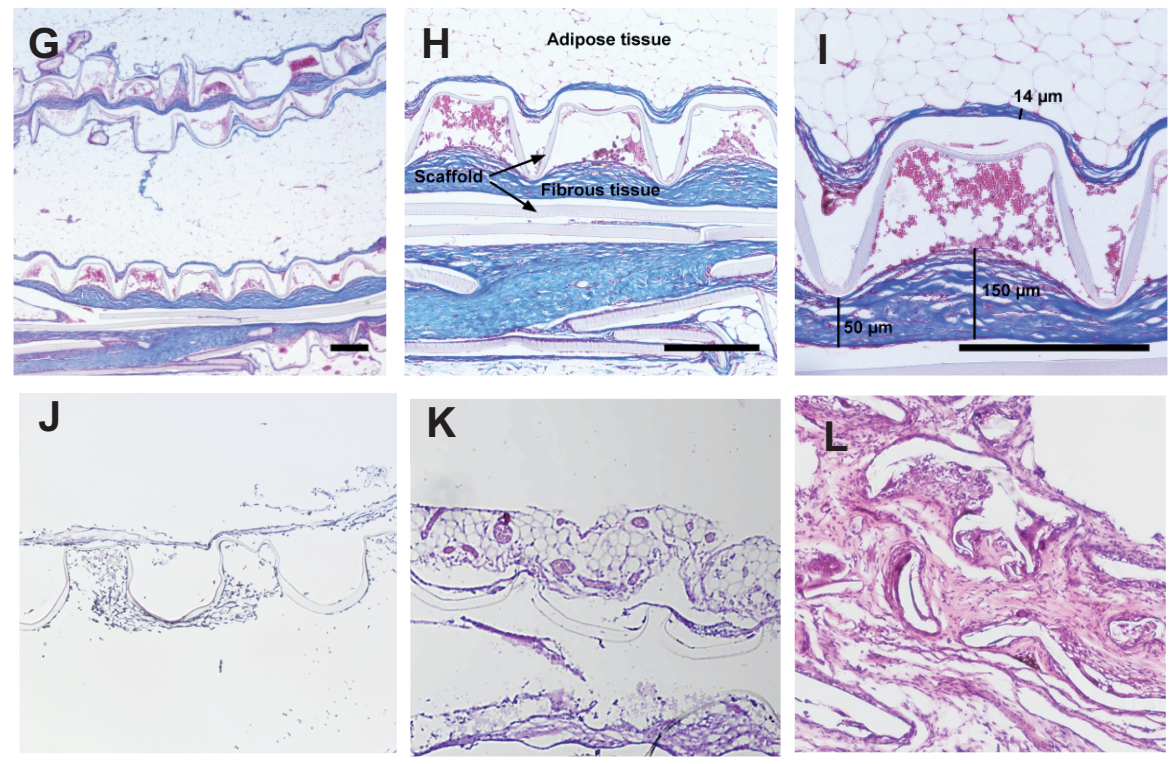

Figure 2. Histology of implanted non-porous microwells scaffolds in subcutaneous and intraperitoneal sites for biocompatibility test: A) A PEOT-PBT scaffold (rat 14) implanted in intraperitoneal site and B) subcutaneous site. C) A poly(ester urethane) scaffold implanted in intraperitoneal site. D-F) HE staining of a PEOT-PBT implant and G-I) trichrome stain in the sagittal direction. J-L) HE stain in the sagittal direction respresent the structure of the poly(ester urethane) at different time points. G) at week 1, K) week 4, I) Week 12. Scale bar indicates $300 \mu \mathrm{m}$.

Both hematoxylin-eosin and trichrome staining (Figure 3 D-F) indicated that implantation of both islet-laden polymer scaffolds led to very low tissue response in the form of macrophage-like cell infiltration. Moreover, blood vessels in growth was observed within the wells. Moreover, vessels successfully grew through the scaffolds micropores in the PEOT-PBT scaffolds (Figure 3 D and F). Positively stained $\beta$-cells were found in about $50 \%$ of PEOT-PBT wells that were implanted in rat number 11 (Figure $3 \mathrm{~J}$-L). Islets did not seem to adhere to the polymer, but were located in the centre of their wells. However, islets could only be detected in $0-10 \%$ of wells in the rest of the PEOT-PBT and poly(ester urethane)-scaffold implanted rats (Figure 3 G-I). In all control animals, islet fusion was observed after 40 days of implantation (Figure 3 M-O). 

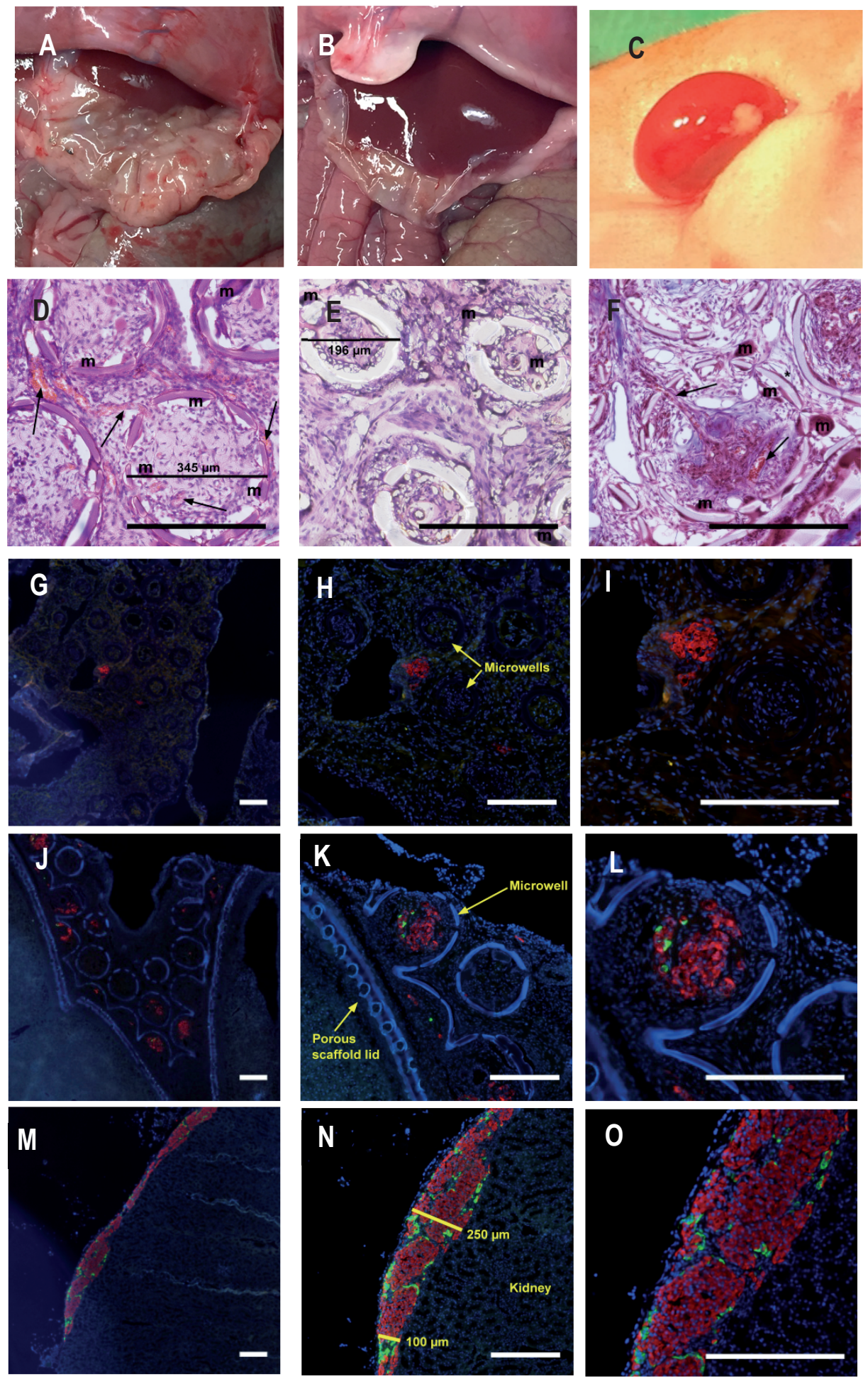
Figure 3. Histology results of islet-seeded scaffold implants. A-C) Show the urethane) and C) kidney control, in which islets are injected under the kidney capsule. Hematoxylin and eosin stainings of tissue-embedded D) (PEOT-PBT) and E) blood vessels (arrows), macrophages (m) and fibrous layer surrounding the implant (*). F) An trichrome stain of PEOT-PBT, which visualizes blood vessels (color) and fibrous structures (color). In addition, IHC was performed for cell nuclei (DAPI, blue), glucagon (green) and insulin (red) and shown G-I) for the poly(ester urethane), J-L) for PEOT-PBT and M-O) for kidney control samples.

\section{Normoglycemia after transplantation}

rats demonstrating the functionality of implanted islets. The blood glucose level progressively reduced from the second day post transplantation onwards and reached normoglycemic levels after 4 days (Figure 4). A stable blood glucose level of $\sim 3.5 \mathrm{mmol} / \mathrm{L}$ was maintained through the study period (>40 days). In contrast, both types of scaffolds demonstrated fluctuation of blood glucose level throughout the study and failed to restore normoglycemia. Regardless of the polymer type in all of the transplanted rats, blood glucose level was higher than $10 \mathrm{mmol} / \mathrm{L}$.

One of the rats that received a poly(ester urethane) scaffold managed to reach a blood glucose level lower than $10 \mathrm{mmol} / \mathrm{L}$ at day 13; and it remained in low level until day 15. However, the reduction of glucose level was not consistent and started to increase 16 days post-implantation (Figure $4 \mathrm{C}$ ). 
A

Control

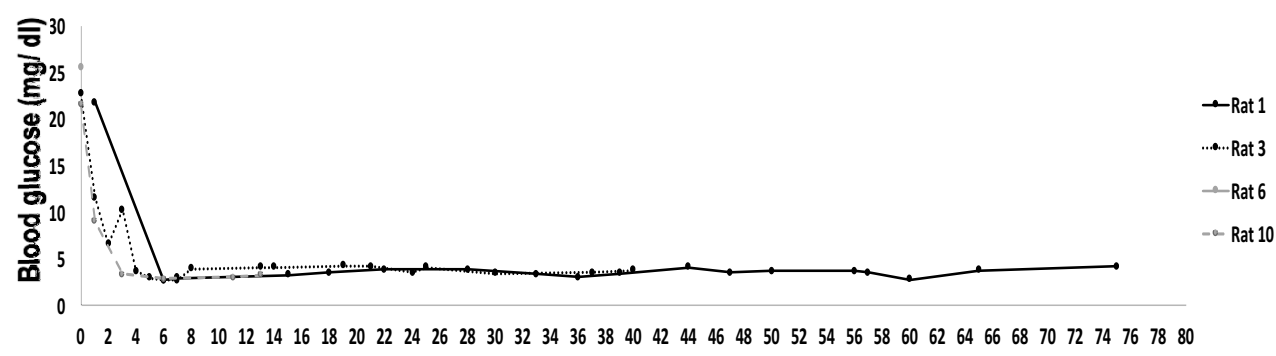

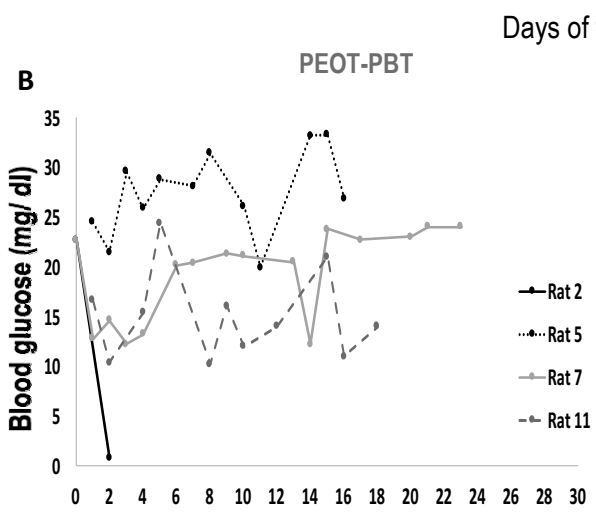

Days of transplantation
C Poly(ester urethane)

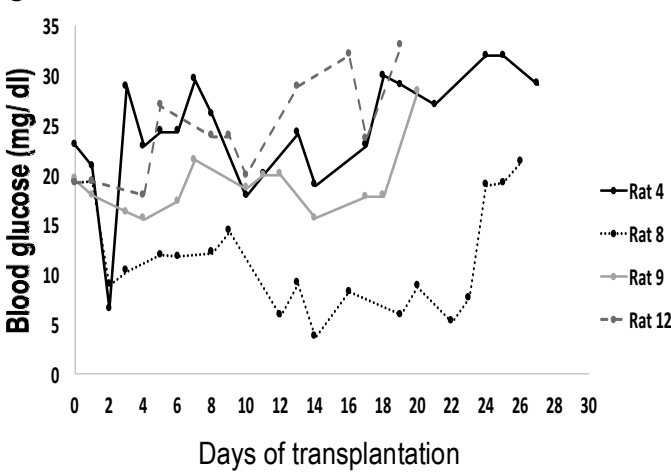

Figure 4. Daily blood glucose level of the rats after islet transplantation under the A) kidney capsule (control), B) and in the PEOT-PBT and poly(ester urethane) scaffolds.

\section{Oral glucose tolerance test (OGTT)}

To evaluate the function of the transplanted islets, an OGTT was performed two weeks after transplantation. The islets that were transplanted under the kidney capsule (control group) demonstrated an immediate response to the glucose rise by releasing insulin. The increase in the insulin level initiated instantly after meal consumption and reached a peak level of $1.67 \mathrm{ng} / \mathrm{ml}$ after 10 minutes. However, variation in insulin release pattern was observed for the PEOT-PBT and poly(ester urethane). The increase in insulin level began immediately after glucose consumption in rat 7 and 11 (transplanted with PEOT-PBT scaffolds) and rat 8 and 
9 (transplanted with poly(ester urethane) scaffolds). In these rats the level of insulin reached a similar value compared to the control group and demonstrated a peak value of approximately $1.5 \mathrm{ng} / \mathrm{ml}$ in $5-10$ minutes. Additionally, the glucose levels of the same rates reached a normoglycemic level in random time intervals, while the control group stayed in normoglycemic level within the test's duration. The insulin:glucose rate of rat 7, 8, 9 and 11 at their insulin peak were about 0.26 , which was almost at the same rate as the control group (0.33). All rats from the control group gained weight $(\sim 29.5 \%)$ and their overall health was improved after transplantation. However, animal treated with encapsulated islets increased in weight $(\sim 11 \%)$ until day 22 after which their weight either remained stable or decreased. The rats displayed a normal behaviour and appearance until day 26.
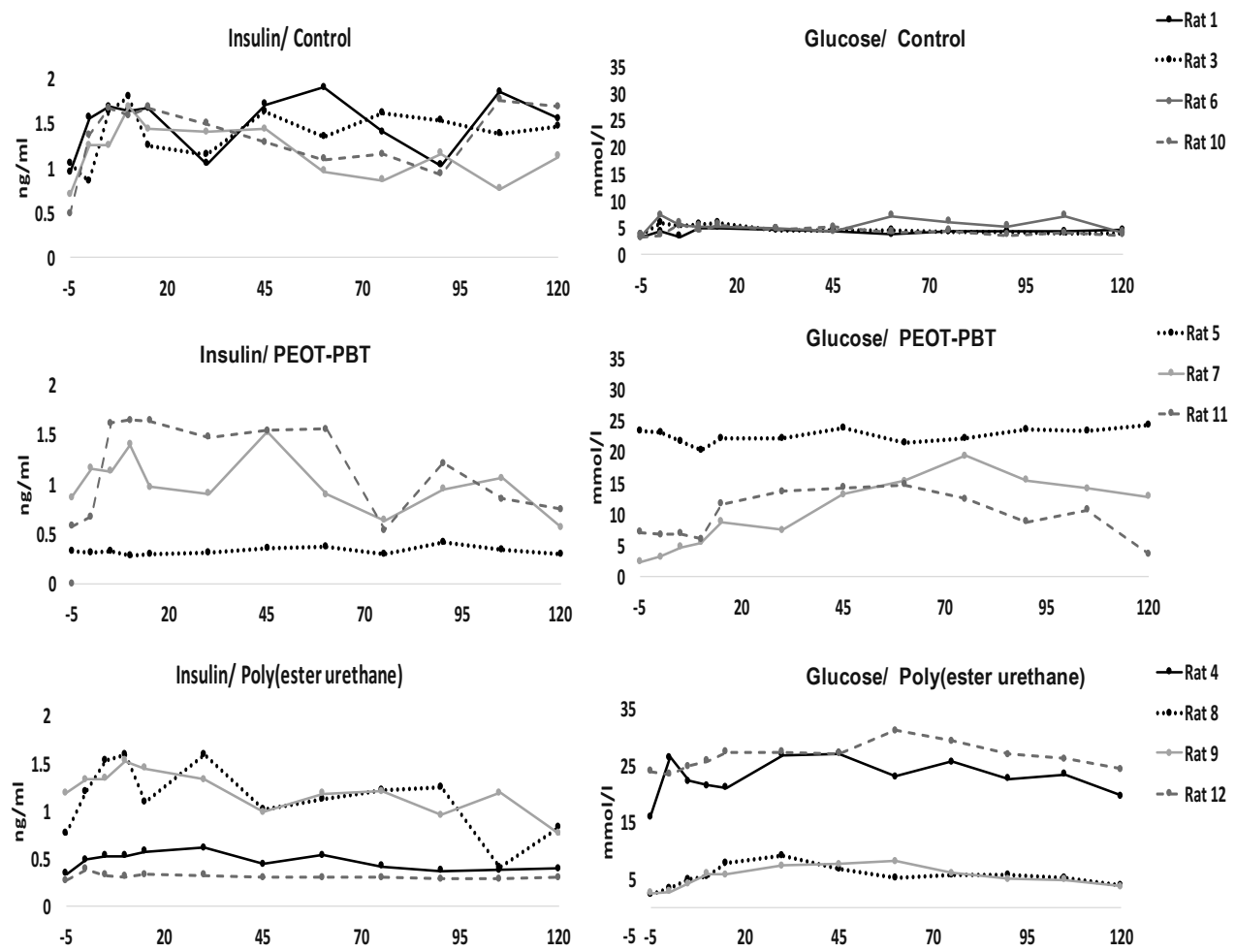

Figure 5. Insulin level during oral glucose tolerance (OGTT) test for the transplanted islets under the kidney capsule (control), PEOT-PBT microwells and poly(ester urethane) microwells after 2 weeks transplantation (within 120 minutes). 


\section{Insulin positive islets}

After 40 days of islet transplantation, the islet-containing kidneys of the control group were carefully excised. Histological evaluation revealed that although a large number of islets were insulin positive, the islets were unorganized, fused together, and lost their spherical shape.

In the scaffold group, considerable number of islets ( $\sim 90 \%)$ were lost likely explain why normoglycemia was not achieved. Interestingly the remaining islets in the scaffold preserved their spherical shape. Additionally, using these scaffolds one of the biggest challenges of islet transplantation, which is islets fusion, was prevented. These results suggest that the porous microwell scaffolds have the potential to overcome the limitations of previous approaches for islet transplantation once the issue of islet loss is solved.

\section{Discussion}

In this study, we explored an approach that could mitigate several drawbacks of macro-encapsulation, such as the selection of a biocompatible material to fabricate macro-encapsulation devices. Specifically, we demonstrated that the microwells allow for the transplantation of a high number of islets to the both subcutaneous and intraperitoneal sites. Our scaffolds possess the advantages of preserving islets morphology by maintaining the native round shape and preventing the aggregation and fusion of islets (Figure 3). The correlation between the function of the islets with their native shape and aggregation size has been proved in several studies [1214]. Disruption of the islets' round structure has been shown to reduce capability of the cells to produce insulin [15]. In addition to shape, it is of particular importance to provide cells of islets with sufficient amount of nutrient. Cell nourishment cannot be achieved if the islets form big aggregates or are fused. The aggregation can result in cell necrosis, specifically in the core part of the aggregate 
due to nutrient diffusion limitations. Microwell scaffolds have the potential to control the size of islets aggregates and protect them from deformation, which consequently might maintain islets function (chapter 5).

To study the biocompatibility of the microwell scaffolds in vivo, empty non-porous microwell scaffolds were implanted in rats. Another group of rats were transplanted with porous microwells laden with islets to evaluate the functionality of implanted islets. Histological assessment of the biocompatibility group indicated that laser drilling of the scaffold is essential to obtain proper tissue integration with the scaffolds (Figure 2). In the absence of micropores, tissue and scaffold form separate layers which impedes biocompatibility analysis. Moreover, the pores facilitated high quality histological analysis by enabling facile sectioning of the constructs. Nevertheless, the coverage of the scaffolds with fat tissues introduced difficulties to perform proper cryotome sectioning and subsequent histological analyses.

Transplanting islets in scaffolds effectively prevented islet aggregation and fusion. In contrast the transplanted islets in the control group clustered together and sometimes individual islets had fused into bigger aggregates (Figure 3 M-O). This observation demonstrated an added value of the microwell array delivery device; it enables islets to retain their morphology and prevent their aggregation. Due to the functional dependence of the islet cells to cell-cell signalling, the islets have a high affinity to form aggregates. However, the dimension of the aggregates can limit the mass transport of nutrients and consequently influence the function and survival of islet cells [16]. Thus, confining the limited number of islets in the structure of scaffolds could prevent the formation of large cell cluster and reduce diffusion restrictions. Nevertheless, the highest number of glucagon- and insulinpositive cells was observed in control group rather than in scaffold group. This might be due to the easy access of the seeded islets under the kidney capsule to the surrounding capillary system, while the penetration of vasculature into microwells requires few days to weeks. 
Normoglycemic levels were reached in most of the rats that were transplanted with the scaffold only until day 2 . Only one of the rats remained normoglycemic for 24 days. Transplanted islets in most of the rats were likely not sufficiently and metabolically supported, which prevented reaching normoglycemia and inevitably led to massive islet loss due to starvation induced cell death. In fact, the islets could be lost during the initial phase of the implantation, which is during the prevascular phase. Moreover, our histology data shows the presence of macrophages, which could suggest the influence of immune reaction in loss of islet mass. However, further investigation is required to confirm affect of immune cells on the transplantation outcome.

Another reason of islet mass reduction can relate to change in the microwell scaffolds structure after transplantation. On the contrary to PEOT-PBT scaffolds that preserved their well structure, the poly(ester urethane) scaffolds lost their primary microwell shape, which is likely due to its relatively soft nature and short degradation time in comparison to PEOT-PBT. Build-up of fibrous tissue and wound contraction could cause the well structures of poly(ester urethane) scaffolds to be pressurized and eventually buckled. Consequently, either the round structure of islets were negatively influenced and thus islets were susceptible to undergo apoptosis or islets were pushed out of their respective wells (Figure 3 G-I).

In the previous study of our group, PEOT-PBT microwell scaffolds were transplanted in the epididymal fat of the BALB/c mice [17]. Unlike the previous study, in which the lid and microwells with the size of $\sim 1 \mathrm{~cm}^{2}$ were attached together by means of 3 sutures, in this study we applied an easier, faster and more controllable method to seal microwells to their lid. We used soldering pen for either fully sealing of scaffolds or partial sealing (in 6 spots) and demonstrated that the efficiency of islet transplantation could be improved by fully sealing of the scaffold to its lid. Sealing scaffold in fewer spots might increase the chance of islet mass loss through the gaps between the sealed spots after transplantation. 
Apart from sealing method, our platform was different from the previous study in the porosity and size of the scaffolds as well as the number of the seeded islets. We believed that higher number of pores and islets in a spacious microwell would not only increase the rate of vascularization, but also would meet the needs of a bigger animal (rat in comparison to mice) in order to reverse diabetes.

The most important difference between these two studies relate to the site of transplantation. In the previous study, the epididymal fat as a highly vascularised site and structurally similar to the greater omentum $[18,19]$ was selected for islet transplantation and they showed that 6 out of 8 animals reverted to stable normoglycemia within 26 days. This data is inconsistent with our current findings, where normoglycemia was observed in the transplanted scaffolds in the intraperitoneal site of rats that were covered with vascularized-fat for few days. In our current study, the intraperitoneal site was chosen because it is large enough to contain a large microwell film to develiver sufficient amounts of islets to achieve normoglycemia in a rat (or human in the future studies). Furthermore, the less intense immune reaction in the intraperitoneal site could protect the transplanted islets from an intense invasion of immune cells. We also chose subcutaneous site of rat for transplantation because it is an easy accessible and retrievable site. Additionally, the presence of intense vasculature in this site could accelerate the ingrowth of vessels into the microwells. However, after transplanting the islets encapsulated in microwell scaffolds in the subcutaneous and intraperitoneal sites, we observed rapid islet mass reduction. This is potentially due to poor vascularization into the scaffolds. Since, the process of islet isolation from a donor damages the vasculature system of islets, the survival of cells mainly depends on the passive diffusion of the necessary nutrients. However, this mechanism cannot fulfil the needs of the cells for long time and consequently in the absence of vascular system, islet cells tend to die. To overcome this limitation, we suggest to optimize our porous microwell platform to promote vascularization. This aim can be achieved either by prevascularization of the scaffolds or by including a growth factor delivery system in the scaffolds. Additionally, functionalizing the scaffolds 
with the extracellular matrix proteins can also enhance the function and improve the survival of encapsulated islets.

\section{Conclusions}

The current study presents the PEOT-PBT microwell scaffold as a suitable carrier for extrahepatic islet transplantation. Based on the results the structure of PEOTPBT microwell scaffolds were stable for 12 week. In contrast, the poly(ester urethane) microwells lost their shape slightly due to the soft nature of the polymer at week 1 and strongly after 4 week. The microwells torn apart after 12 weeks implantation. Both types of scaffolds proved sufficiently biocompatible. Moreover, the immune reaction in subcutaneous site was slightly higher than intraperitoneal site. After transplantation of islets using both types of scaffold, we did not observe consistent normoglycemia. In addition, our finding demonstrated loss of islet mass shortly after transplantation. Based on our results, an insufficient vascularization rate might be the main reason for the reduction islets number and function. Further investigation is necessary to optimize the scaffold and improve the outcome of islet transplantation using microwell scaffolds. 


\section{References}

1. Rathmann, W. and Giani, G. (2004) Global prevalence of diabetes: estimates for the year 2000 and projections for 2030. Diabetes care 27 (10), 2568-2569.

2. Cade, W.T. (2008) Diabetes-related microvascular and macrovascular diseases in the physical therapy setting. Physical therapy 88 (11), 1322.

3. Paty, B.W. et al. (2002) Intrahepatic islet transplantation in type 1 diabetic patients does not restore hypoglycemic hormonal counterregulation or symptom recognition after insulin independence. Diabetes 51 (12), 3428-3434.

4. Ricordi, C. (2003) Lilly Lecture 2002: islet transplantation: a brave new world.(Perspectives in Diabetes). Diabetes 52 (7), 1595-1604.

5. Moberg, L. et al. (2002) Production of tissue factor by pancreatic islet cells as a trigger of detrimental thrombotic reactions in clinical islet transplantation. The Lancet 360 (9350), 2039-2045.

6. Biarnés, M. et al. (2002) $\beta$-cell death and mass in syngeneically transplanted islets exposed to short-and long-term hyperglycemia. Diabetes 51 (1), 66-72.

7. Davalli, A.M. et al. (1996) Vulnerability of islets in the immediate posttransplantation period: dynamic changes in structure and function. Diabetes 45 (9), 1161-1167.

8. Carlsson, P.-O. (2011) Influence of microenvironment on engraftment of transplanted $\beta$-cells. Upsala journal of medical sciences 116 (1), 1-7.

9. Buitinga, M. et al. (2013) Microwell scaffolds for the extrahepatic transplantation of islets of Langerhans. PloS one 8 (5), e64772.

10. Truckenmüller, R. et al. (2011) Thermoforming of film-based biomedical microdevices. Advanced materials 23 (11), 1311-1329.

11. Barkai, U. et al. (2013) Enhanced oxygen supply improves islet viability in a new bioartificial pancreas. Cell transplantation 22 (8), 1463-1476.

12. Kim, A. et al. (2009) Islet architecture: a comparative study. Islets 1 (2), 129136.

13. Cabrera, O. et al. (2006) The unique cytoarchitecture of human pancreatic islets has implications for islet cell function. Proceedings of the National Academy of Sciences of the United States of America 103 (7), 2334-2339.

14. Reaven, E. et al. (1981) Effect of variations in islet size and shape on glucosestimulated insulin secretion. Hormone and Metabolic Research 13 (12), 673-674.

15. Kelly, C. et al. (2011) Role of islet structure and cellular interactions in the control of insulin secretion. Islets 3 (2), 41-47.

16. Nam, K.-H. et al. (2010) Size-based separation and collection of mouse 
17. Buitinga, M. et al. (2017) Micro-fabricated scaffolds lead to efficient remission of diabetes in mice. Biomaterials 135, 10-22.

18. Salvay, D.M. et al. (2008) Extracellular matrix protein-coated scaffolds promote the reversal of diabetes after extrahepatic islet transplantation. Transplantation 85 (10), 1456.

19. Gibly, R.F. et al. (2013) Porous scaffolds support extrahepatic human islet transplantation, engraftment, and function in mice. Cell transplantation 22 (5), 811-819. 



\section{Chapter 7}

Endothelial growth factor delivery systems from assembled polymer nanolayers

E. Hadavi

F.F.R. Damanik

C.M. Bruinik

J. Leijten

L. Moroni

A.A. van Apeldoorn

H.B.J. Karperien 


\section{Abstract}

A widely studied and applied method to reconstitute glucose homeostasis in diabetes mellitus is clinical islet transplantation. The islet isolation process from donor tissue disrupts amongst others the connectivity and function of the islets' blood vessels. Upon implantation of the isolated islets, lack of nutrients and oxygen due to deficiency in vascularization results in a major loss of islet mass. Re-integrating islets with a functional circulatory system may affecting the secretory cell function, final metabolic regulation and survival of islets. Implants currently studied for the transplantation of islets may be improved when allowing fast blood vessel ingrowth. In this study, we set a solution to increase the rate of blood vessel ingrowth. This was achieved by means of a controlled growth factor releasing system on polymer films. Our currently applied biomaterial scaffolds for clinical islet transplantation were used to deposit growth factors. Oxygen plasma treated PEOT-PBT and poly(ester urethane) polymer films were either coated with vascular endothelial growth factor (VEGF) and heparin or a self-assembled layer by layer (LBL) system composed of poly(ethylene imine) (PEI), poly(styrene sulfonate) (PSS), heparin, and VEGF. The heparin-VEGF system released a very low amount of growth factors during the first week $(<1 \mathrm{ng} / \mathrm{ml})$ which gradually increased the following 9 days. A constant release of growth factor from the PEOT-PBT and poly(ester urethane) films coated with the LBL system was observed (>2.17 $\mathrm{ng} / \mathrm{ml}$ ). The delivery of VEGF from both coatings significantly increased endothelial tube formation in vitro compared to non-coated or no VEGF containing control scaffolds. Coated polymer films also presented longer tube lengths as compared to the control groups at day 14. Based on these results, the presented LBL system has a growth factor release profile that is highly suited to stimulate the vascularization of the transplanted islets using biomaterial scaffolds. 


\section{Introduction}

Islets of Langerhans require a rich blood supply for the delivery of oxygen, nutrients and the distribution of secreted hormones to their target organs [1]. The islet isolation process from donor tissue requires enzymatic digestion, which results in disruption of islets from their extracellular matrix and surrounding blood vessels. Re-implanted islets are strongly dependent on the long-range diffusion of nutrients and oxygen from the host's vascular system. This limits the survival of cells in the center of the islets and causes a rapid loss of islet mass $[2,3]$. Therefore, reestablishing an adequate microcirculation to the re-implanted isolated islets is necessary to increase their survival rate and functional behavior.

Angiogenesis is a complex process consisting of pericellular proteolysis, migration and proliferation of endothelial cells as well as sprouting, formation of capillaries and maturation of blood vessels. Angiogenesis is stimulated by activation of signaling pathways that have receptors for hypoxia driven growth factors. Since in the transplanted islets the oxygen tension is lower than in native islets, hypoxic conditions could lead to secretion of vessel regulatory growth factors such as vascular endothelial growth factor (VEGF) and basic fibroblast growth factors (bFGF) [4]. However, revascularization of transplanted islets only occurs approximately 10 days after transplantation, which usually is too late to maintain cell viability.

Several studies have focused on the enhancement of vascularization following islet transplantation. Different strategies have been explored, such as delivery of growth factors, expression of angiogenic genes as well as the use of vascularinductive cell types. Genetic overexpression of VEGF [5-8], angiopoietin-1 [9] or thrombospondin-1 [29] was shown to improve the vascularization of the transplanted islets in rodents. Despite the promising results of this technique, the clinical application of it is limited due to safety considerations associated with gene transfer. Moreover, it is reported that overexpression of angiogenic genes in islets 
may cause hyper-vascularization and enhanced inflammation leading to an adverse effect on $\beta$-cell function $[10,11]$.

Co-culturing of pro-angiogenic cells, such as mesenchymal stem cells with islets, enhances islet vascularization by expression of high levels of VEGF. Moreover, these cells contribute to the activation of the signaling pathway in islet endothelial proliferation, differentiation, and angiogenesis [12-15]. However, this strategy appears of limited use due to the clinical complexity of delivering cells from multiple sources as well as its high costs.

Protein-based angiogenic growth factor delivery methods contribute to a safe and simple strategy to improve vascularization [16-18]. This strategy has a high potential to improve the outcome of islet transplantation when using biomaterial scaffolds with low safety concern.

Different strategies have been developed to control the release of growth factors from hydrogels $[19,20]$, microspheres [21, 22], or covalent immobilization of growth factors to biomaterials [23]. Another method applied is the electrostatic binding of growth factors to heparin-sulfate or heparin [24, 25]. Heparin is a natural component of the ECM and has a high affinity for electrostatic binding of growth factors including VEGF and FGF-2 [26, 27]. This ability provides stabilization of the active conformation of growth factors and facilitates their use in release systems [26, 28].

VEGF and bFGF have an important role in the vascularization process of islets. VEGF is a critical regulator of neovascularization that is used for clinical application [29]. The receptors of VEGF, including flk-1 and flt-1, are expressed in healthy islets and are up-regulated in hypoxic or de-vascularized islets [30]. VEGF-mediated angiogenesis stimulates the production of metalloproteinases (MMPs), which leads to degradation of the surrounding ECM, migration of endothelial cells and formation of buds and sprouts [31]. Some studies indicated that both endothelial cells of the host tissue and donor islets are involved in the revascularization process. It was indicated that endothelial cells remaining in isolated islets, used for 
transplantation purposes, retain a significant potency to develop tubule-like structures [32]. Thus, for effective therapeutic angiogenesis and establishment of a stable and functional vascular network, precise dosing of growth factors by temporal or spatial delivery to the cells and control over their release are important factors to consider.

In previous research, we developed polymeric devices potentially applicable for the transplantation of islets. Both a polyester based poly(ethylene oxide terephthalate) and poly(butylene terephthalate) (PEOT-PBT) and the poly(ester urethane) were used to encapsulate islets in microwell porous scaffold for islet transplantation. To increase the efficiency of islet transplantation, it is necessary to accelerate vascularization in these devices. For this aim, we recently studied the influence of a VEGF-heparin coating as well as a novel self-assembled multilayer, prepared by layer by layer deposition (LBL), on vascularization. In the first method polymer films were coated by VEGF and heparin. In the second method, a layer-by layer (LBL) self-assembled coating composed of layers of poly(ethylene imine) (PEI), poly(sodium 4-styrenesulfonate) (PSS), heparin, and VEGF was applied. An in vitro tube formation assay was performed to evaluate biological function of the released VEGF for each coating over time. These controlled growth factor delivery systems may be applied to the microwell scaffolds to improve the function and survival of islets by accelerating revascularization after transplantation.

\section{Materials and methods}

\section{Polymer film fabrication}

The 4000PEOT30PBT70, abbreviated as PEOT-PBT was produced by Polyvation BV (Groningen, the Netherlands, under the trade name PolyActive ${ }^{\mathrm{TM}}$ ). The copolymer is composed of poly(ethylene oxide terephthalate) blocks with a PEO molecular weight $(\mathrm{Mw})$ of 4000 and the weight percentage (wt\%) of PEOT and 
poly(butylene terephthalate) (PBT) blocks was $30 \mathrm{wt} \%$ and $70 \mathrm{wt} \%$, respectively [33]. The poly(ester urethane) was provided by Polyganics BV (Groningen, the Netherlands). It was synthesized by reaction of an $a, \omega$-isocyanate end functionalized pre-polyester and an a,w-hydroxy bis-carbamate (Chapter 3). Chloroform (Merck, Darmstadt, Germany) and 1,1,1,3,3,3-hexafluoro-2isopropanol (Biosolve, Valkenswaard, the Netherlands) were used as solvents.

Thin films with a thickness of $40 \mu \mathrm{m}$ were fabricated using solvent casting. The PEOT-PBT was dissolved in a 65:35 (w/w) mixture of chloroform and 1,1,1,3,3,3hexafluoro-2-isopropanol at a concentration of $15 \mathrm{wt} \%$ and casted on silicon wafers at room temperature. The polymer films were dried under a continuous nitrogen stream for $4 \mathrm{~h}$ followed by immersion in ethanol to remove residual solvent and easily peeling off from the substrate. Subsequently, the polymer films were dried in a vacuum oven (Heraeus, Hanau, Germany) at $30^{\circ} \mathrm{C}$ for 3 days. Poly(ester urethane) films with a thickness of $40 \mu \mathrm{m}$ were fabricated in a similar way from a $5 \mathrm{wt} \%$ polymer solution in chloroform. Circular specimens of $1 \mathrm{~cm}$ in diameter were punched from both PEOT-PBT and poly(ester urethane) films.

\section{Deposition of a VEGF/heparin coating}

The surfaces of the polymer discs were treated (for 5 min, each side) with an oxygen plasma (Tetske, Nanolab, University Twente) at a pressure of 100 mTorr and power of $100 \mathrm{~W}$. The polymer films were incubated in $250 \mu \mathrm{l}$ of $80 \mathrm{ng} / \mathrm{ml}$ VEGF in $0.1 \%$ BSA (pH 5) for $20 \mathrm{~min}$ and thereafter in $1 \mathrm{mg} / \mathrm{ml}$ heparin in $\mathrm{PBS}(\mathrm{pH} 9)$ for $10 \mathrm{~min}$. This cycle was performed 4 times. After each step incubation with heparin and VEGF, discs were rinsed with milliQ water and PBS for 1 minute, respectively.

\section{Deposition of multilayer thin films}

Multilayer thin coatings were deposited on PEOT-PBT and poly(ester urethane) polymer discs by the LBL assembly method. Both sides of the polymer discs were treated with an oxygen plasma (Tetske, Nanolab, University Twente) at a pressure 
of $100 \mathrm{mTorr}, 100 \mathrm{~W}$, and $50 \mathrm{sccm} \mathrm{O}_{2}$ for $5 \mathrm{~min}$. For the deposition of the layers the following solutions were prepared; $2 \mathrm{mg} / \mathrm{ml}$ PEI in milliQ water ( $\mathrm{pH} 3$ ), 2 $\mathrm{mg} / \mathrm{ml}$ PSS in milliQ water ( $\mathrm{pH} 10), 1 \mathrm{mg} / \mathrm{ml}$ heparin in PBS (pH 9) and $80 \mathrm{ng} / \mathrm{ml}$ VEGF in 0.1 bovine serum albumin (BSA) ( $\mathrm{pH} 5)$.

The surface of ultra-low attachment multiwell plates was coated with $2 \%$ BSA overnight to prohibit non-specific binding on the surface of the plates. The treated PEOT-PBT and poly(ester urethane) polymer discs were placed in the wells and incubated with $250 \mu \mathrm{l}$ of solutions in the following sequence; PEI; PSS; PEI; PSS. Then 4 times alternating with VEGF and heparin and then a final layer of PEI (Figure 1). The discs were rinsed for $1 \mathrm{~min}$ with milliQ water after each PEI, PSS and heparin incubation step. After incubation with VEGF solution, discs were rinsed with PBS for 1 minute.

\section{Ellipsometry}

The thickness of each layer deposited by the VEGH-heparin and LBL method was determined using an M-2000UI ellipsometer (JA Woollam Co, Lincoln, NE, USA). The principle of ellipsometer measurement is based on the reflection of the exposed beam. Since poly(ester urethane) or PEOTPBT polymer discs did not reflect the beam, silicon wafers were used instead. Silicon wafers were treated for 5 min with an oxygen plasma (TePla300, PVA Tepla AG, Germany) at a power of $300 \mathrm{~W}, 350 \mathrm{sccm} \mathrm{\textrm {O } _ { 2 }}$ at $0.76 \mathrm{mbar}$. Deposition of VEGF/heparin and LBL assembling were performed on silicon wafers, using the same procedure for polymer discs.

\section{VEGF immobilization efficiency and release profile}

VEGF immobilization on VEGF-heparin and LBL coated PEOT-PBT and poly(ester urethane) discs ( $1 \mathrm{~cm}$ in diameter) was determined by measuring the amount of VEGF left in the immobilization medium after each VEGF coating step. For determining the release profile, discs were immersed in $1 \mathrm{ml}$ of PBS containing $0.1 \% \mathrm{BSA}$ at $37^{\circ} \mathrm{C}$ for 14 days. Every day the solutions were removed and placed in 
low binding microcentrifuge tubes (Eppendorf) that were coated overnight with $2 \%$ BSA. The solutions removed were replaced with $1 \mathrm{ml}$ of PBS, containing $0.1 \%$ BSA. The released VEGF was quantified using a Quantikine VEGF ELISA kit (R\&D Systems) according to the manufacturer's instructions.

\section{Cell culturing}

HUVECs primary cells were cultured in endothelial cell basal medium (EBM-2) supplemented with the EGM-2 BulletKit (Lonza) at $37^{\circ} \mathrm{C}$ in humidified air containing $5 \% \mathrm{CO}_{2}$. In all experiments HUVECs were used up to passage 5.

\section{In vitro tube formation assay}

An in vitro tube formation assay served as a model to qualitatively determine the influence of the release system on the angiogenic activity of endothelial cells. The polymer discs of $1 \mathrm{~cm}$ in diameter were placed in $\mu$-slide 8-well plates (Ibidi, Munich, Germany). GeltrexTM reduced growth factor basement membrane matrix (Invitrogen) was thawed at $4^{\circ} \mathrm{C}$ overnight to prevent premature polymerization. The GeltrexTM matrix (150 $\mu \mathrm{l} /$ well) was added to each well and allowed to polymerize at $37^{\circ} \mathrm{C}$ for $1 \mathrm{~h}$. HUVEC cells (40,000 cells/well) were seeded onto the GeltrexTM matrix together with $250 \mu \mathrm{l}$ of EBM medium. As control groups EBM supplemented with $2 \%$ FBS and 0, 1, 2, or $4 \mathrm{ng} / \mathrm{ml} \mathrm{VEGF}$ was used. Endothelial cell growth medium-2 (EGM-2; Lonza) was used as a positive control. For each heparin-VEGF and LBL group EBM media with supplements but without any growth factor was used. HUVECs endothelial cells were incubated up to $48 \mathrm{~h}$ after 1, 7, and 14 days to allow the formation of tube-like structures. The extent of tube formation at 10 random fields per well was photographed using an EVOS microscope (Advanced Microscopy Group, Bothell, WA) after 4, 8, 24 and 48 hours. Tube formation was quantified by measuring the length of tubes, tube numbers and tube mesh (loops) in random fields using ImageJ. 


\section{Results}

\section{Ellipsometry}

In order to create a controlled VEGF releasing system, two methods were investigated. Polymer discs from punched PEOT-PBT and poly(ester urethane) films were plasma treated with oxygen to create a negatively charged surface. In a first method, alternating incubation steps of VEGF and heparin solutions were applied to deposit a coating on the substrates. In the second method, layer by layer (LBL) deposition of positively charged PEI and negatively charged PSS was used to create multiple layers on the polymer discs. Then, the discs were alternatingly incubated with a VEGF and heparin solution and finally covered with a PEI layer (Figure 1). To evaluate the thickness of each deposited layer using ellipsometry. As the polyester and poly(ester urethane) were incompatible with the process a silicon wafer was used as a model substrate. The silicon wafer was plasma treated with oxygen to similarly create a negatively charged surface. By repeated addition of VEGF-heparin bilayers, the thickness remained unchanged after the first incubation step with VEGF (Figure 1). In the LBL deposition method a linear increase in thickness of the first 4 layers of alternating PEI and PSS was observed. However, the thickness after addition of the bilayers of VEGF-heparin did not change possibly due to interpenetration of VEGF and heparin molecules in the PEI and PSS layers. By deposition of the final PEI layer an increase in thickness was observed. In detail, the total thickness of multilayers was $9.44 \pm 0.41 \mathrm{~nm}$ and thickness of layers up to first layer of PEI, PSS and VEGF were 0.86 $\pm 0.07,3.10 \pm 0.16$ and 8.92 $\pm 0.37 \mathrm{~nm}$, respectively. The same molecular structure and thickness of VEGF-heparin layer in LBL system observed in VEGF-heparin bilayers coated directly on silicon with the thickness of $2.67 \pm 0.11,3.30 \pm 0.10$ and $3.57 \pm 0.22 \mathrm{~nm}$ for initial layer of VEGF, bilayer of VEGF-heparin and total coated layer, respectively (Figure 1). 


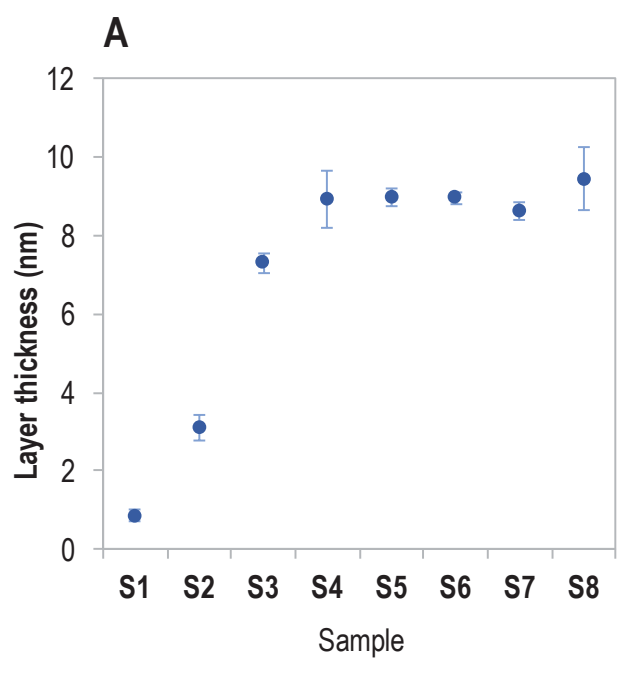

C



LBL

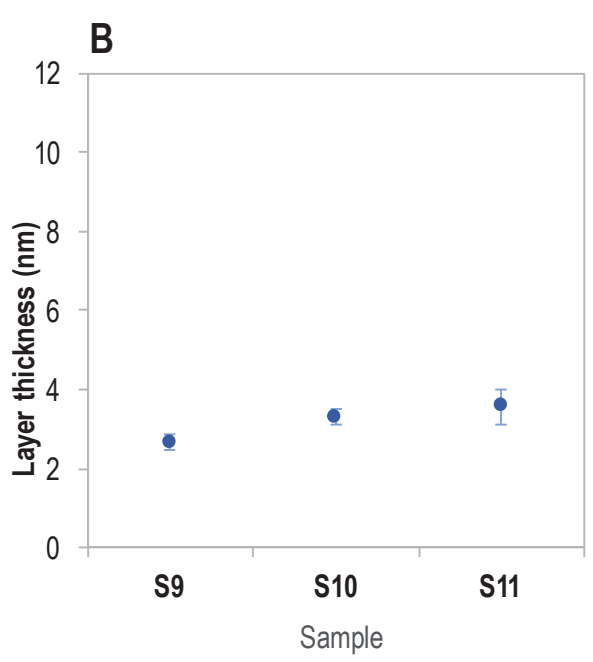

O VEGF

$\square$ Heparin

PSS

PEI

Silicon wafer

\section{VEGF-Heparin}

Figure 1. Thickness of the layers deposited on a silicon wafer substrate following the LBL and VEGF-heparin deposition methods and as determined ellipsometry. A) Thickness of LBL deposited layers after incubation steps (S); S1:PEI, S2: PSS, S3: PEI, S4: VEGF, S5: heparin, S6: VEGF, S7: heparin and S8: PEI B) Thickness of VEGH-heparin immobilized on a surface of silicon; S9: VEGF, S10: heparin and S11: after 4 times alternating incubation with VEGF and heparin $\mathbf{C}$ ) schematic illustration of the LBL and VEGF-heparin deposition.

\section{VEGF immobilization efficiency and release profile}

Upon multiple incubation steps with VEGF and heparin solutions the thickness of the layers did not increase after the first deposited layer. However, the loading efficiency of the VEGF in the first layer was very effective on both PEOT-PBT and poly(ester urethane) films and revealed to be approximately $89 \%$ and $92 \%$ applying the VEGF-heparin and LBL deposition methods, respectively. In detail, the VEGF loading on the PEOT-PBT and poly(ester urethane) discs, was 72.4 2.6 and $71.6 \pm 2.9 \mathrm{ng} / \mathrm{cm}^{2}$, respectively, applying the VEGF-heparin deposition method. The loading efficiency of VEGF on the PEOT-PBT and poly(ester urethane) films using 
the LBL method was $74.3 \pm 2.3$ and $73.9 \pm 1.9 \mathrm{ng} / \mathrm{cm}^{2}$, respectively (Figure $2 \mathrm{~A}$ and B).

The release of VEGF from VEGF-heparin coated polymer films was less than 0.7\% up to 9 days and increased after that exponentially up to $\sim 4 \%$ on day 14 . In detail, the observed VEGF release was less than $0.3 \mathrm{ng} / \mathrm{ml}$ for both the PEOT-PBT and poly(ester urethane) coated films by VEGF-heparin up to about 9 days. The low release of VEGF from these coatings indicated a strong binding between VEGF and plasma treated polymer films. Release may be dependent on the degradation of the heparin (Figure 2C and D). An almost constant release was observed for the LBL multilayers coated PEOT-PBT and poly(ester urethane) polymer films. Over a period of 14 days the release increased from 3 to $5 \mathrm{ng} / \mathrm{ml}$.
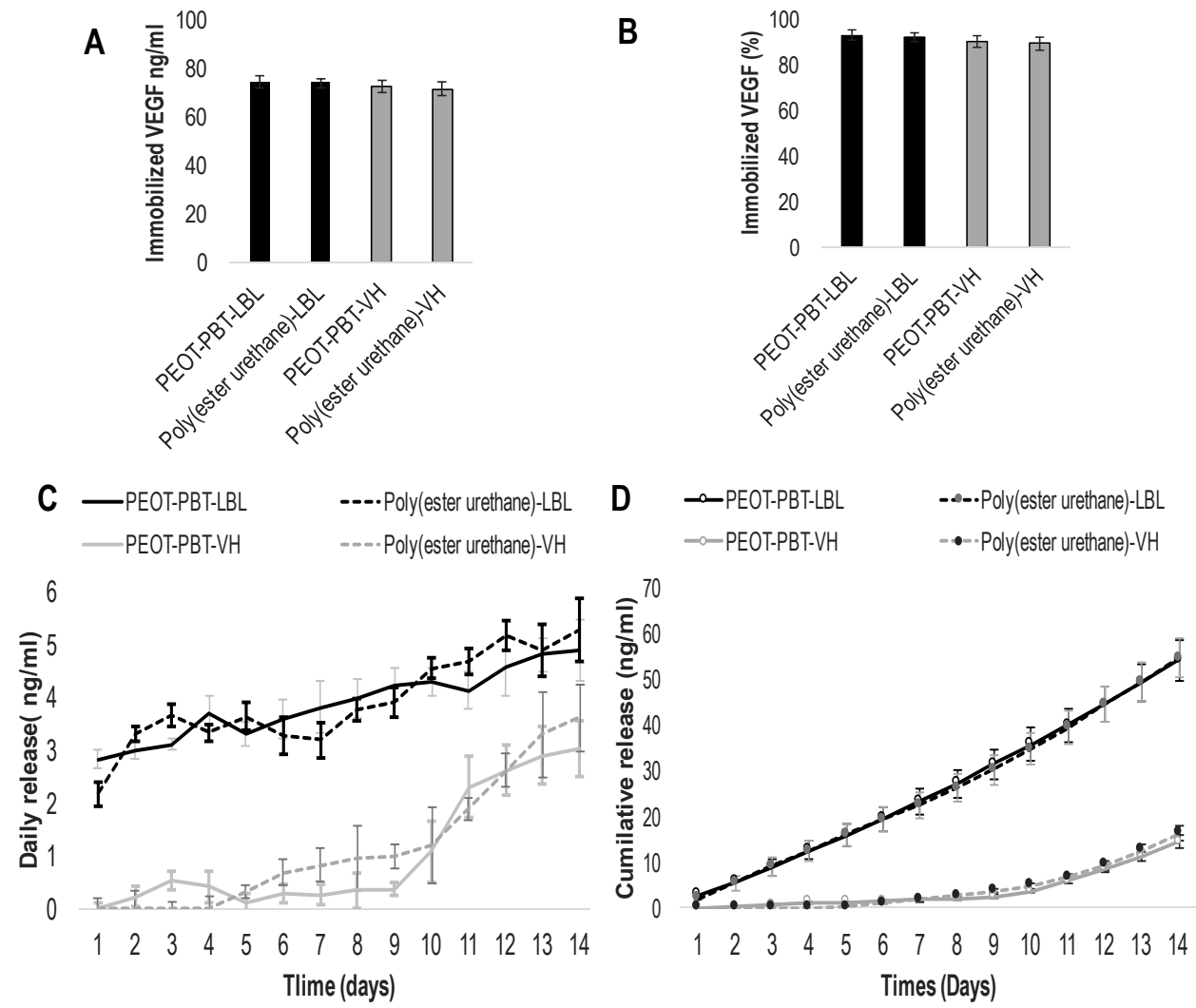

Figure 2. A) Immobilized amount of VEGF on (PEOT-PBT -LBL), (Poly(ester urethane)LBL), (PEOT-PBT-VEGF/Heparin) and (Poly(ester urethane)-VEGF/Heparin). B) Percentage of the immobilized VEGF C) Daily release of VEGF D) Cumulative release of VEGF. 


\section{Tube formation}

An in vitro tube formation assay was applied to qualitatively evaluate the influence of the coatings deposited on the PEOT-PBT and poly(ester urethane) polymer films on the angiogenic activity of endothelial cells.

At day 1,7 and 14 media containing the VEGF released in the last 48 hours was used to perform a tube forming assay. Based on the results, at day 1, 7 and 14 the average tubular length and mesh number of the LBL groups were higher than of the control group (EGM as a medium) and dramatically higher than the control group without growth factors (Figure 3). Monitoring the release process for 48 hours after initiation of tube formation at each time point indicated that the tubular length slightly decreased after 24 hours, however, the tubular length remained constant up to 48 hours (Figure S1). The tubular length after 48 hours was higher in the LBL groups than all control groups with $1 \mathrm{ng} / \mathrm{ml}, 2 \mathrm{ng} / \mathrm{ml}, 4 \mathrm{ng} / \mathrm{ml}$ VEGF in EBM media, EBM media without VEGF and EGM media. These data indicated the efficiency of the sustained VEGF release on proliferation of the endothelial cells by means of the LBL system and subsequent formation of a tubular network. The tubular length might be consistent or even higher in the case of daily refreshment of the media in LBL group. These data matched with the number of meshes (loops) after tube formation.

The tube length and mesh number for both polymer films coated with VEGFheparin at day 1 and 7 was almost the same as the control group without any growth factors (Figure 3). However, evaluating the tubular length of for PEOT-PBT group at day 1 and 7 as well as poly(ester urethane) group at day 7 indicated a gradual increase of tube length which was even higher than that found for the EGM media control group (Figure S1). These data revealed that the release of VEGF at low concentrations already have a positive effect on HUVECs proliferation and tubular formation. The tubular length and mesh numbers after 14 days for all groups were dramatically higher than those of the control group without growth factor and somewhat higher than those of the control groups with EGM and $4 \mathrm{ng} / \mathrm{ml}$ 
VEGF (Figure 3). Evaluating the tubular formation at day 14 for 48 hours indicated higher tubular length in all groups as compared to both control groups with EGM or $4 \mathrm{ng} / \mathrm{ml}$ VEGF (Figure $\mathbf{S} 1$ ).

A Total Tube Length after 8 hours

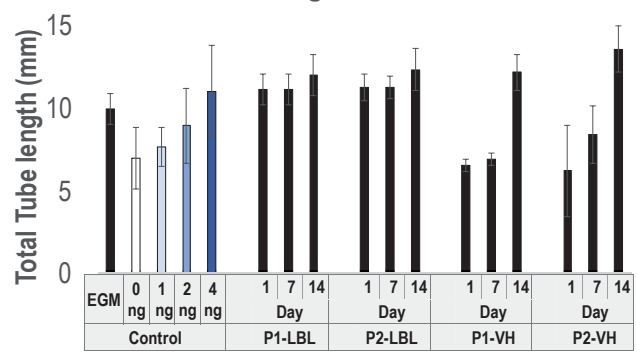

B Tube numbers after 8 hours

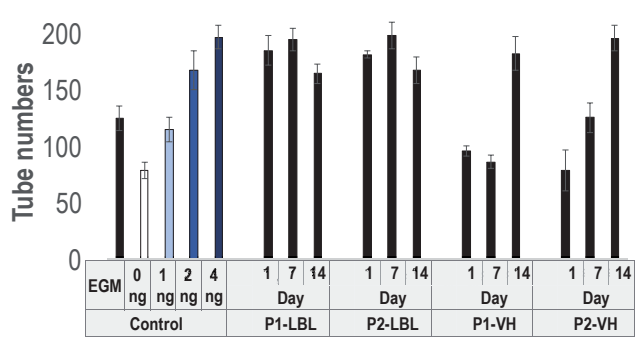

C

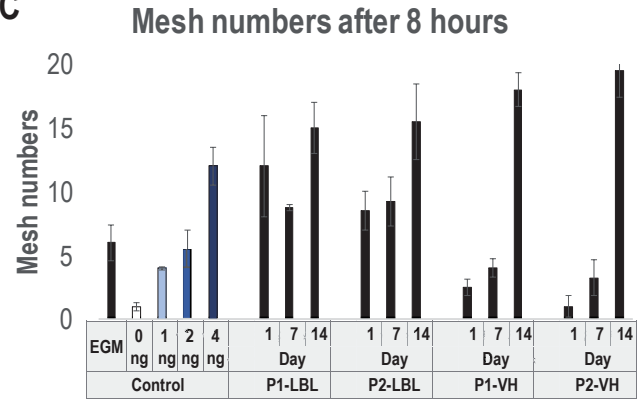

Total Tube Length after 48 hours



Tube numbers after 48 hours
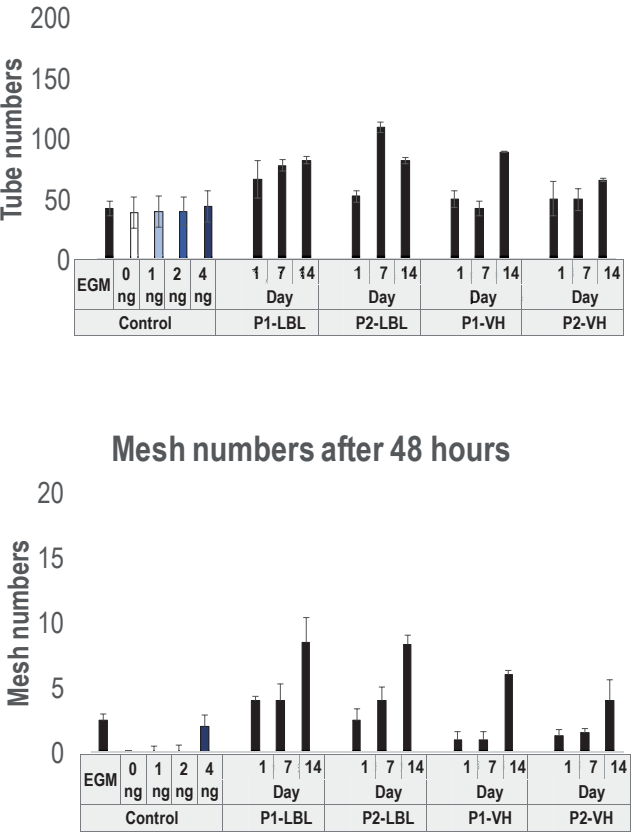

Figure 3. Tube formation of VEGF stimulated by EGM, $0,1,2$ and 4 ng VEGF as control groups, and VEGF relesing systems including PEOT-PBT-LBL (P1-LBL), Poly(ester urethabe)-LBL (P2-LBL), PEOT-PBT-VEGF-Heparin (P1-VH) and Poly(ester urethabe) (P2-VH) at day 1,7 and 14. A) total tube length B) Tube numbers and C) mesh (loop) numbers after 8 and 48 hours. 


\section{Discussion}

One of the main obstacles for clinical islet transplantation is the lack of vascularization immediately following transplantation, which adversely affect the function and survival of islets. Acceleration of vascularization after transplantation and connection of the new vasculature to the systemic circulation, in order to provide adequate oxygen levels and nutrients to islets, could improve the outcome of clinical transplantation. In this study, we present a novel self-assembled multilayer deposition, prepared by a layer by layer (LBL) technique, to immobilize VEGF on PEOT-PBT and poly(ester urethane) polymers films. We hypothesized that such coatings would yield a sustained release of VEGF in time. We expect that this approach may accelerate vascularization of islet in implants i.e. by depositing these coatings on the lid of the microwell scaffolds used in the transplantation of islets [ref chapter 6].

Important factors to be considered in the development of successful growth factor delivery systems and regulate cell behavior are the loading efficiency, release and prohibition of a high initial burst release [34]. To develop suitable VEGF releasing systems, heparin and VEGF were used due to their positive role in improving vascularization. Moreover, it is known that heparin binds to different angiogenic growth factors including FGF-2 [35] and VEGF [4], via their heparin binding domains. A high loading efficiency of VEGF in both systems, due to both the generated negative charge on the substrates and high affinity between heparin and VEGF, was achieved. This binding also protects growth factors from enzymatic degradation [36], which is important in enhancement of cell signaling in new blood vessel formation [37]. It has been reported that heparin is likely to form a complex with VEGF-A and its receptor VEGFR-2 which results in increasing the VEGF-Ainduced phosphorylation of VEGFR [38].

Based on previous studies, surface charge of biomaterials could be used to grow multilayers on their surfaces [39]. Plasma oxidation treatment of polymer surfaces appeared a suitable method to introduce a negative charge on the surface of PEOT- 
PBT and poly(ester urethane) polymer films. Using a layer by layer assembly technique, by immersion of the polymer films in sequential solutions of positively and negatively charged polymers, allows the deposition and built up of a homogenous layer. Compared to non-LBL systems including gas deposition, nucleation deposition this technique is highly suitable for thin film formation on polymer surfaces.

Using ellipsometry we tried to determine the thickness of the deposited layers of PEI and PSS on the surface of the polyester and poly(ester urethane) discs. However, these polymer discs appeared not suitable for ellipsometry. In contrast to the polymer films, silicon is not transparent at the wavelengths of interest (i.e. UVVIS region), so there are no backside reflection issues. Silicon also has a very high refractive index, so the contrast with transparent materials e.g. mono- and multilayers is high. The native $\mathrm{SiO}_{2}$ layer thickness is very uniform and allows activation by $\mathrm{O}_{2}$ plasma for LBL assembly of polyelectrolytes[40]. Therefore, we used silicon wafers as a model system. Depositing VEGF and after that heparin to the silicon wafer surface showed the formation of a layer with a thickness of $\sim 2.7$ nm (Figure 1). Attempts to increase the thickness of this layer by immersion in VEGF and heparin solutions was not successful. In the LBL system sequential layers of PEI and PSS (3.6 nm) were deposited first. The thickness of PEI and PSS layers $(2.3 \mathrm{~nm})$ at pH 6 was similar as reported in a study by Elzbieciak et al [40]. Addition of sequential VEGF and heparin to the deposited layer for 4 times showed that the thickness of the layer did not change which might be representative of an interpenetration of heparin and VEGF molecules in the sublayer. Subsequently, after addition of a final PEI layer, the thickness increases, showing the deposition of a membrane covering the heparin VEGF layer. In spite of the small changes in thickness for the VEGF-heparin deposition using both the VEGF-heparin and LBL systems it was found that approximately $80 \mathrm{ng}$ of VEGF was incorporated in the layer as determined by depletion of the incubation solution.

The release profiles of VEGF from the different coatings were notably different. VEGF release of VEGF-heparin coated polymer films was very low during the first 
7 days, which may be due to the strong binding of VEGF and the negatively charged PEOT-PBT and poly(ester urethane) polymer surfaces. The VEGF release exponentially increased after 9 days, which might be due to degradation of heparin layer. In contract, a constant VEGF release was observed from the LBL coating. A gradual increase was determined in the daily release (Figure 1). Diffusion is regulated and limited by the PEI membrane on top of the VEGF-heparin layer in this system.

Comparing the VEGF release profile and tube formation process for all groups indicated that the tube length almost matches the data of the VEGF daily release. The release profile of VEGF from VEGF-heparin coated polymer films was very low $(<0.3 \mathrm{ng} / \mathrm{ml})$ at day 1 which resulted in tube length lower than the control group without any growth factors. The increase in the VEGF release of $\sim 3 \mathrm{ng} / \mathrm{ml}$ at day 14, showed an increase in the tube length to the same level as the control group with $\sim 4 \mathrm{ng} / \mathrm{ml}$ VEGF. For the LBL group the VEGF release starts from $\sim 3 \mathrm{ng}$ at day 1 and increases up to $\sim 5 \mathrm{ng}$ at day 14 . An almost similar tube length is found compared to the control group with $\sim 4 \mathrm{ng} / \mathrm{ml}$ VEGF. The tube length for all LBL groups (at day 1, 7 and 14) after 48 hours is higher than tube length in the control group with $4 \mathrm{ng} / \mathrm{ml}$ VEGF which indicated the efficiency of the LBL releasing system. Based on the results, the LBL system formed even higher tubular length, more tube and mesh number compare to the EGM positive control group. It should be noted that tube forming assays are not truly predictive for in vivo angiogenesis. Regardless, the observation of an almost release of VEGF for coatings on both PEOT-PBT and poly(ester urethane) polymer films suggested that the LBL method might be transferable to other polymers as well. This system has a high potential for application on improvement of vascularization after islet transplantation.

Our data suggested that upregulation of VEGF-induced tube formation as well as proliferation of HUVECs was correlated with heparin binding. This phenomenon could be leveraged to enhance angiogenesis for tissue engineering applications. 


\section{Conclusion}

This study indicated that VEGF immobilization in VEGF-heparin coatings is a convenient strategy for protection of VEGF. The LBL method provided a sustained release of VEGF while the VEGF-heparin system resulted in an initial delayed release from coatings on PEOT-PBT and poly(ester urethane) polymer films. The LBL assembly system represents a new approach for improving vascularization. 


\section{Supplemental Material}

A -- EGM ……ng - $1 \mathrm{ng} \quad-2 \mathrm{ng}-4 \mathrm{ng} \quad$-P1-LBL -- P2-LBL -P1-VH --P2-VH
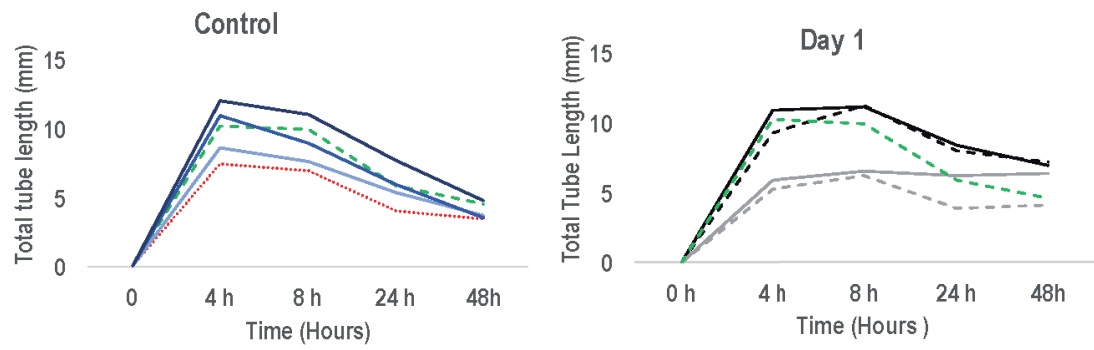

Day 7
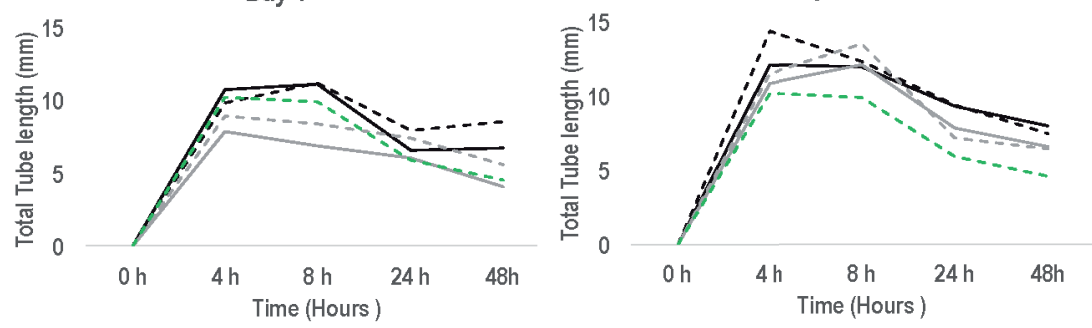

B
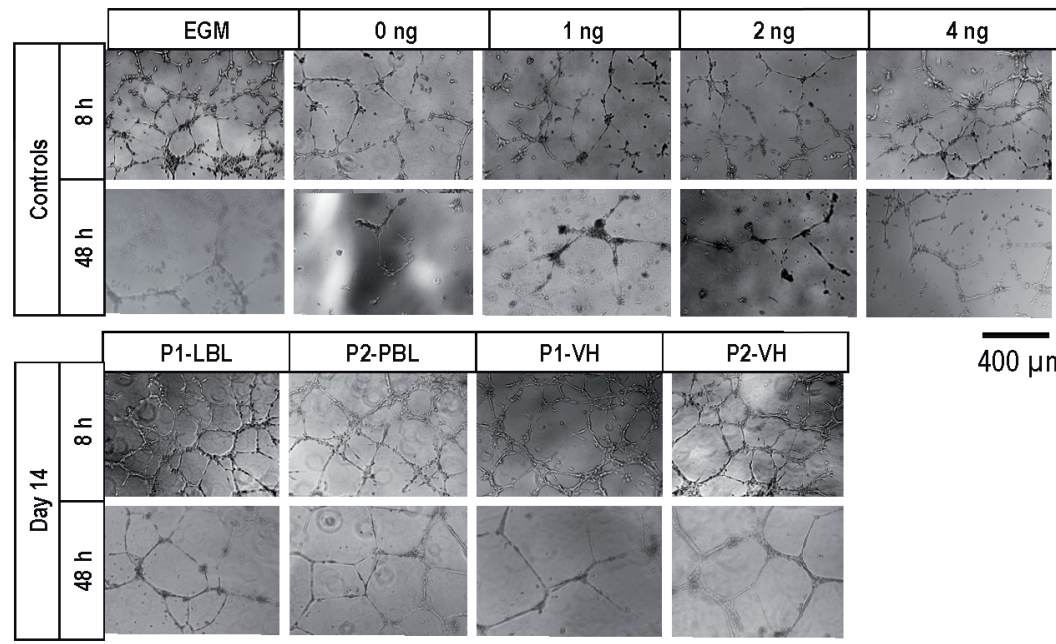

$\overline{400 \mu \mathrm{m}}$

$400 \mathrm{um}$

Supplemental figure 1. Tube formation of VEGF stimulated by EGM, 0 , 1, 2 and 4 ng VEGF as control groups, and VEGF relesing systems including PEOT-PBT-LBL (P1-LBL), Poly(ester urethabe)-LBL (P2-LBL), PEOT-PBT-VEGF-Heparin (P1-VH) and Poly(ester urethabe) (P2VH) whitin 48 hours at day 1,7 and 14. A) Total tube lengh, B) Representative phase contrast images of tube formation for control groups and VEGF relesing systems at day 14, after 8 and 48 hours. 


\section{References}

1. Brunicardi, F.C. et al. (1996) Microcirculation of the Islets of Langerhans: Long Beach Veterans Administration Regional Medical Education Center Symposium. Diabetes 45 (4), 385-393.

2. Brandhorst, H. et al. (1999) SIGNIFICANT PROGRESS IN PORCINE ISLET MASS ISOLATION UTILIZING LIBERASE HI FOR ENZYMATIC LOWTEMPERATURE PANCREAS DIGESTION1. Transplantation 68 (3), 355-361.

3. Dionne, K.E. et al. (1993) Effect of hypoxia on insulin secretion by isolated rat and canine islets of Langerhans. Diabetes 42 (1), 12-21.

4. Neufeld, G. et al. (1999) Vascular endothelial growth factor (VEGF) and its receptors. The FASEB journal 13 (1), 9-22.

5. Cheng, K. et al. (2004) Adenovirus-based vascular endothelial growth factor gene delivery to human pancreatic islets. Gene therapy 11 (14), 1105.

6. Narang, A.S. et al. (2004) Vascular endothelial growth factor gene delivery for revascularization in transplanted human islets. Pharmaceutical research 21 (1), $15-25$.

7. Cheng, Y. et al. (2007) Elevation of vascular endothelial growth factor production and its effect on revascularization and function of graft islets in diabetic rats. World Journal of Gastroenterology: WJG 13 (20), 2862.

8. Langlois, A. et al. (2011) Improvement of rat islet viability during transplantation: validation of pharmacological approach to induce VEGF overexpression. Cell transplantation 20 (9), 1333-1342.

9. Su, D. et al. (2007) Angiopoietin-1 production in islets improves islet engraftment and protects islets from cytokine-induced apoptosis. Diabetes 56 (9), 2274-2283.

10. Agudo, J. et al. (2012) Vascular endothelial growth factor-mediated islet hypervascularization and inflammation contribute to progressive reduction of $\beta$ cell mass. Diabetes 61 (11), 2851-2861.

11. Cai, Q. et al. (2012) Enhanced expression of VEGF-A in $\beta$ cells increases endothelial cell number but impairs islet morphogenesis and $\beta$ cell proliferation. Developmental biology 367 (1), 40-54.

12. Cavallari, G. et al. (2012) Mesenchymal stem cells and islet cotransplantation in diabetic rats: improved islet graft revascularization and function by human adipose tissue-derived stem cells preconditioned with natural molecules. Cell transplantation 21 (12), 2771-2781.

13. Sakata, N. et al. (2011) Utility of co-transplanting mesenchymal stem cells in islet transplantation. World Journal of Gastroenterology: WJG 17 (47), 5150. 
14. Yeung, T.Y. et al. (2012) Human mesenchymal stem cells protect human islets from pro-inflammatory cytokines. PloS one 7 (5), e38189.

15. Cantaluppi, V. et al. (2012) Microvesicles derived from endothelial progenitor cells enhance neoangiogenesis of human pancreatic islets. Cell transplantation 21 (6), 1305-1320.

16. Stendahl, J.C. et al. (2009) Extracellular matrix in pancreatic islets: relevance to scaffold design and transplantation. Cell transplantation 18 (1), 1-12.

17. Sigrist, S. et al. (2003) Influence of VEGF on the viability of encapsulated pancreatic rat islets after transplantation in diabetic mice. Cell transplantation 12 (6), 627-635.

18. Chow, L.W. et al. (2010) Self-assembling nanostructures to deliver angiogenic factors to pancreatic islets. Biomaterials 31 (24), 6154-6161.

19. Moon, J.J. et al. (2010) Biomimetic hydrogels with pro-angiogenic properties. Biomaterials 31 (14), 3840-3847.

20. Lee, K.Y. and Yuk, S.H. (2007) Polymeric protein delivery systems. Progress in polymer science 32 (7), 669-697.

21. Perets, A. et al. (2003) Enhancing the vascularization of three-dimensional porous alginate scaffolds by incorporating controlled release basic fibroblast growth factor microspheres. Journal of Biomedical Materials Research Part A 65 (4), 489-497.

22. Young, S. et al. (2005) Gelatin as a delivery vehicle for the controlled release of bioactive molecules. Journal of controlled release 109 (1), 256-274.

23. Chiu, L.L. et al. (2011) Defining conditions for covalent immobilization of angiogenic growth factors onto scaffolds for tissue engineering. Journal of tissue engineering and regenerative medicine 5 (1), 69-84.

24. Zieris, A. et al. (2010) FGF-2 and VEGF functionalization of starPEG-heparin hydrogels to modulate biomolecular and physical cues of angiogenesis. Biomaterials 31 (31), 7985-7994.

25. Pike, D.B. et al. (2006) Heparin-regulated release of growth factors in vitro and angiogenic response in vivo to implanted hyaluronan hydrogels containing VEGF and bFGF. Biomaterials 27 (30), 5242-5251.

26. Taipale, J. and Keski-Oja, J. (1997) Growth factors in the extracellular matrix. The FASEB Journal 11 (1), 51-59.

27. Ono, K. et al. (1999) Structural features in heparin that interact with VEGF165 and modulate its biological activity. Glycobiology 9 (7), 705-711.

28. Ruhrberg, C. et al. (2002) Spatially restricted patterning cues provided by heparin-binding VEGF-A control blood vessel branching morphogenesis. Genes \& development 16 (20), 2684-2698. 
29. Ferrara, N. and Kerbel, R.S. (2005) Angiogenesis as a therapeutic target. Nature 438 (7070), 967.

30. Christofori, G. et al. (1995) Vascular endothelial growth factor and its receptors, flt-1 and flk-1, are expressed in normal pancreatic islets and throughout islet cell tumorigenesis. Molecular Endocrinology 9 (12), 1760-1770.

31. Richardson, T.P. et al. (2001) Polymeric system for dual growth factor delivery. Nature biotechnology 19 (11), 1029-1034.

32. Linn, T. et al. (2003) Angiogenic capacity of endothelial cells in islets of Langerhans. The FASEB journal 17 (8), 881-883.

33. Buitinga, M. et al. (2013) Microwell scaffolds for the extrahepatic transplantation of islets of Langerhans. PloS one 8 (5), e64772.

34. Lee, K. et al. (2011) Growth factor delivery-based tissue engineering: general approaches and a review of recent developments. Journal of the Royal Society Interface 8 (55), 153-170.

35. Nugent, M.A. and Iozzo, R.V. (2000) Fibroblast growth factor-2. The international journal of biochemistry \& cell biology 32 (2), 115-120.

36. Saksela, O. et al. (1988) Endothelial cell-derived heparan sulfate binds basic fibroblast growth factor and protects it from proteolytic degradation. The Journal of cell biology 107 (2), 743-751.

37. Abhinand, C.S. et al. (2016) VEGF-A/VEGFR2 signaling network in endothelial cells relevant to angiogenesis. Journal of cell communication and signaling 10 (4), 347-354.

38. Ashikari-Hada, S. et al. (2005) Heparin regulates vascular endothelial growth factor165-dependent mitogenic activity, tube formation, and its receptor phosphorylation of human endothelial cells comparison of the effects of heparin and modified heparins. Journal of Biological Chemistry 280 (36), 31508-31515.

39. Peng, C. et al. (2011) Effect of precursor-layer surface charge on the layerby-layer assembly of polyelectrolyte/nanoparticle multilayers. Langmuir 28 (1), 84-91.

40. Elzbieciak, M. et al. (2009) Influence of $\mathrm{pH}$ on the structure of multilayer films composed of strong and weak polyelectrolytes. Langmuir 25 (5), 32553259. 

Chapter 8

Conclusion and outlook 


\section{Conclusion}

A considerable number of people suffer from type 1 diabetes. Although the systematic application of exogenous insulin prolongs the lifetime of millions of diabetic patients, it is associated with inevitable side effects and thus reduces their quality of life. Currently, an alternative for treatment of type 1 diabetes is pancreatic islet transplantation, which may result in insulin independency of patients for several years. However, islet failure shortly after transplantation, insufficient insulin secretion and shortage of donors are major obstacles for this therapy. The main objective of the research described in this thesis was to improve the outcome of islet transplantation. By mimicking the islet's native microenvironment and exploring various bioengineering strategies devices were developed that could be applied for the transplantation of islets.

\section{Comprehensive review on the islet's native microenvironment}

A key reason for islet transplantation failure is the isolation-induced damage to the islet microenvironment. To set the stage for this thesis, we have provided a comprehensive review on the biological, biochemical and biophysical characteristics of the islet microenvironment and the relation of these parameters with islets function and survival in chapter 2 . This understanding will aid the development of an ideal engineered islet and includes the essential aspects of natural pancreatic islets such as extracellular matrix (ECM)-cell interaction, cellcell interaction, islet aggregate size, and vasculature network. In addition, biomaterial choice, scaffold architecture, and immunoprotection techniques play an important role in the engineering of a functional islet microenvironment. Despite the progress in the field of islet transplantation, current methods are still unable to successfully mimic the microenvironment of human islets. 
With the aim of coming up with an alternative transplantation technique, we focused on the engineering of the islets' microenvironment by applying tissue engineering approaches. We set our goal to enhance the survival and functional performance of islets' after transplantation by simulating the natural microenvironment and morphology of pancreatic islets as well as stimulating islet vascularization.

\section{Biomaterial properties for islets engineering}

The first experimental step described in this thesis was the selection of an appropriate biomaterial for islet encapsulation. In chapter $\mathbf{3}$, we highlighted the required biomaterial properties to be considered in the design of scaffolds. This included; coinciding biomaterials' degradation time with their intended function, possessing suitable mechanical properties and being biocompatible. A major consideration for islet engineering is; whether a long-lasting scaffold preserving the islet shape and function would be desired, or whether a short-aged biomaterial, that permits the islets to adapt to the grafted sites microenvironment, would be preferred. With this dilemma in mind, we compared the physical and mechanical properties of the poly(ester urethane) and 4000PEOT30PBT70 in relation to their degradation. The poly(ester urethane) was synthesized by reaction of an a,wisocyanate end functionalized pre-polyester and an a,w-hydroxy bis-carbamate, and 4000PEOT30PBT70 was composed of poly(ethylene oxide terephthalate) and poly(butylene terephthalate) blocks which abbreviated as PEOT-PBT. The in vitro degradation was followed at $\mathrm{pH} 5,7.4$ and 9 at $37^{\circ} \mathrm{C}$ over a period of 34 weeks. The physical properties of these materials largely depended on the molecular structure of the polymer chains. The PEOT-PBT polymer is hydrophobic and semicrystalline and slowly degrades in physiological conditions. The shape of PEOT-PBT films was retained up to 34 weeks, although the film's surface became irregular and showed some cracks. Unlike the PEOT-PBT, a shorter degradation time was observed for the poly(ester urethane), which was coincident with previous findings 
[1-3]. Furthermore, the poly(ester urethane) rapidly became brittle within 5 weeks and showed the characteristics of a bulk degradable material with some increase in crystallinity due to hydrogen bonding of remaining carbamate-containing segments. Based on our findings, we propose that the PEOT-PBT material can be used to create stable three-dimensional tissue engineered scaffolds for transplantation of islets for long-term use. However, the poly(ester urethane), due to its short degradation time can be useful for the support of islets for a short period of time until they become compatible with the transplanted site's microenvironment. Subsequently, we analyzed the ECM-cell interaction to study the islets transplantation outcome.

\section{ECM pattering to biomimic the islet microenvironment}

When engineering tissues, the ECM is of particular importance due to its structural and biochemical supportive roles [4] as well as its correlation with cellular processes including proliferation, differentiation, adhesion, and cell-cell communication [5-7]. In pancreatic islets, Collagen (Col), Fibronectin (FN), and Laminin (LN) are the most important ECM molecules that exist in the basement membrane and stromal ECM of islets [8]. Interaction of these ECM molecules with islet cells (e.g. $\beta$-cells) has demonstrated to improve the survival rate and function of cells both in vitro and in vivo $[9,10]$. However, previous works have mainly focused on evaluating islet cells, in particular $\beta$-cells, cultured on scaffolds with conventionally coated ECM molecules. Such a uniform ECM coating cannot optimally simulate and support the natural morphology of cells and could even negatively affect the $\beta$-cells physiology and function [11].

To explore the effect of ECM molecules and to improve the function and viability of INS-1E cells, we immobilized FN, Col4, and LN111 molecules on PDMS by microcontact printing (chapter 4). Microcontact printing makes it feasible to have a defined coated pattern, which both restricts the cells to a predetermined sized 
area and allows to study the interaction with the coated molecules. Using this technique, we created homogeneous and uniform arrays of circular FN, Col4, and LN111 spots of $100 \mu \mathrm{m}$ in diameter on a poly(dimethyl siloxane) substrate. Our findings demonstrated that cultivation of INS-1E cells on these spots coated with ECM molecules, in marked contrast to conventional uniform coatings, resulted in spontaneous cellular self-assembly into islet-like clusters. This 3D biomimetic microenvironment resulted in an improved INS-1E cells insulin secretion.

Based on our findings we concluded that microcontact printing represents a useful technique to study the cluster size dependent behavior of $\beta$-cells. In addition, this method can improve the outcome of islet transplantation by developing implants that provide biomimetic microenvironments. In the following parts, we focused on transferring our findings from single ECM molecules patterning assessments to a more effective and complex combinatorial ECM coated platform to come up with an optimal model for islet transplantation.

\section{The influence of ECM molecules on islet function by a combinatorial approach}

The main focus of past research was the assessment of the interaction of cells with individual ECM molecules or extraction of all ECM molecules from a specific tissue or cultured cells. However, a thorough study on the combined influence of ECM molecules on islets was lacking. Previous studies on the influence of various ECM molecules on islets function and morphology indicated that each molecule impacts islets' differently. For instance, islet adhesion was induced by Col and FN and not by LN. In chapter 5, our hypothesis was that the combination of ECM molecules, each with its own mode of action could further improve the islets function and survival. Therefore, we coated tissue culture plates (TCPs) with pairwise combinations of FN, Col4 and various kinds of laminins at different ratios and explored the islets responses. This study was not limited to coated flat TCP surfaces. Since the disruption of islets microanatomy after seeding can negatively 
affect islet function, we also aimed to simulate the structure and composition of native islet microenvironment by developing microwell scaffolds (chapter 5).

\section{Microwell scaffolds for islet engineering}

As described in chapter 5, we developed a microwell scaffold platform to not only act as a mechanical support and preserve the native morphology of islets, but also to maximize nutrient diffusion, while islet perfusion is kept at a minimum. In this chapter we indicated the beneficial effect of PEOT-PBT and poly(ester urethane) scaffolds, with their specific microwell structures, mechanical properties and low adhering properties, on morphology and function of the islets. Since, our findings in chapter 4 demonstrated enhanced functional performance of INS-1E cells due to induced islet-like structures, we expected that the microwell scaffolds may also improve insulin release by preserving their round shape.

Thus, using PEOT-PBT and poly(ester urethane) polymers we engineered microwell scaffolds comprising separate containers each to encapsulate a single islet. Moreover, other studies reported that the spreading of the islets due to islet attachment to TCP as well as disruption of the islet anatomy by dispersion negatively affect the insulin secretory response [12-14]. However, normal insulin release could be reversed by re-aggregation of the dispersed islet cells which indicates the importance of the cell-cell interaction on insulin release $[12,13,15]$. In addition to islet adhesion and loss of islets native morphology, fusion of islets is associated with loss of insulin secretion [16-19].

\section{Islet engineering via ECM coated microwell scaffolds}

In chapter 5, to mimic the islet's native microenvironment, microwell scaffolds were coated with FN, Col4, as well as various laminins solely and pairwise at 
different ratios. The islets morphology, viability and function after culturing on TCP as well as coated microwell scaffolds were monitored.

Improved survival, enhanced function and the preserved round structure of islets were prominent on TCP and microwells coated with Col4:LN111 at a 8:2 ratio in comparison to the other ratios. Notably, while others reported that only LN does not have any enhancing effects on islets [20], we revealed that the percentage of LN111 mixed with other ECM molecules plays a significant role on insulin secretion. Based on our results, lower than 50\% LN111 combined with FN and Col4 leads to a decrease in insulin secretion. In contrast, higher that $80 \% \mathrm{LN}$ mixed with another ECM molecule provided a stable insulin release for 7 days. When comparing islets cultured in TCP and islets encapsulated in microwells, the highest level of insulin release was observed for the encapsulated islets. We believe this might be due to preservation of the morphology of the islets in the microwells. Finally, by encapsulating individual islets into separate microwell containers, we could overcome a major challenge of macroencapsulation, which is islet fusion. In general, we concluded that the microwell polymeric scaffolds with combinations of ECM molecules could serve as an efficient platform for islet transplantation.

\section{Selection of an islet transplantation site}

Although graft vascularization is of essential importance, the revascularization in the grafted islets starts only several days after transplantation. This temporally limits the nutrient and oxygen availability to the islets as it is fully dependent on the diffusion capacity of the applied scaffold [21]. Using a fully closed scaffold with low diffusion capacity thus hampers metabolites, oxygen and nutrient exchange. In addition, such a system also impedes vascular infiltration. With these limitations in mind, we managed to fabricate microwell scaffolds with high degree of porosity and controlled pore size that can serve as artificial transplantation sites (chapter 6). 
Following profoundly the mechanical properties of the PEOT-PBT and poly(ester urethane) polymers films (chapter 3), we used these polymers to fabricate porous microwell scaffolds that can prohibit islet fusion as well as stimulate islet revascularization.

These scaffolds were fabricated with a defined pore size (10 to $40 \mu \mathrm{m}$ ) using an UV-laser drilling device. Considering the promising results of in vitro experiments these scaffolds could provide a mechanically protective environment and maintain the islets morphology and function in vivo. We first assessed the biocompatibility of the porous microwell scaffolds implanted in the intraperitoneal and subcutaneous site of Albino Oxford (AO) rats. The results indicated minor immune reactions for PEOT-PBT and poly (ester urethane) polymers at both transplanted sites over 12 weeks. The immune reaction at the subcutaneous site was slightly higher than at the intraperitoneal site. At the intraperitoneal site, most scaffolds were covered by vascular-fat tissue from the first week and the thickness of the fat tissue was increasing over time until week 12. In contrast, the microwells at the subcutaneous site were not covered with vascular-fat tissue. Considering the negligible immune reaction and vascular-fat tissue formation that were seen in the intraperitoneal site rather than subcutaneous site, we selected the intraperitoneal site for the following step.

\section{Evaluating the islet transplantation site in vivo}

The ultimate goal of this study was to evaluate the potential of our fabricated scaffolds to induce normoglycemia in diabetic rats. Thus, as presented in chapter 6, the encapsulated islets in the porous microwell scaffolds were transplanted at the intraperitoneal site of diabetic nude Rowett rats and blood glucose levels were measured. Based on our findings, normoglycemia was achieved in none of the rats over 2 weeks and almost all of the islets were lost. Inadequate vascularization of the transplanted islets, which leads to lack of nutrients and oxygen in addition to 
minor immune reaction due to the biomaterial might be the reasons of islet mass loss. In addition, the minor immune reaction induced by the biomaterial could also result in apoptosis of the transplanted islet.

Investigating the structural characteristics of islets and their supportive scaffolds, we observed that the PEOT-PBT microwells stayed well-structured and consequently the scaffolds preserved the round structure of the islets. In contrast, the poly(ester urethane) could not support the islets morphology shortly after transplantation. This might be due to the lower mechanical properties of the poly(ester urethane) compared to the PEOT-PBT.

\section{Vascularization of implants}

Based on the study described in the previous chapter, in the next step of this research, we focused on one of the most important obstacles in islet transplantation, which is implant vascularization. Since human pancreatic islets have a dense vascular network, insufficient vasculature results in hypoxia and eventually reduces islets viability and function [22]. In addition, to provide cells with oxygen and nutrients, the vascular system has a regulatory effect on the endocrine function of islets. The endocrine function of islets depends on the blood flow in and around their microenvironment to adequately sense changes in blood glucose concentrations and efficiently secrete the endocrine hormones. Therefore, it is of particular importance to stimulate vascularization of the transplanted islets and circumvent related problems. One of the most efficient ways to induce vascularization is to apply a controlled release system of growth factors.

As described in chapter 7, in an initial study we developed two systems for the delivery of growth factors from poly(ester urethane) and PEOT-PBT polymer films with the aim of accelerating the islet revascularization. We designed and compared a homogenous coating of heparin and VEGF and a novel layer by layer platform with control over the release of vascular endothelial growth factor (VEGF). The 
multilayer construct consisted of poly(ethylene imine) (PEI), poly(sodium 4styrenesulfonate) (PSS), heparin, and VEGF. The efficiency of the layer by layer system was assessed in vitro and was based on endothelial tubule formation of human umbilical vein cells. Our findings indicated sustained release of the growth factor from both polymers films with a PEI/PSS/heparin/VEGF coating for 2 weeks. Interestingly, the VEGF-heparin coated polymer films demonstrated delayed release of VEGF for more than a week. As a consequence of sustained release of the growth factor from PEI/PSS/Heparin/VEGF coated polymers, the endothelial tubes' length, loops and nodes remained stable over 2 weeks and in case of VEGF-Heparin coated films, tube length increased after about 9 days. Based on the results, heparin immobilization appeared a convenient strategy for protection of VEGF from degradation. The LBL self-assembly of both PEOT-PBT and poly(ester urethane) polymer films provided a controlled release of VEGF, while the VEGF-heparin system resulted in a delayed release. Since immediate oxygenation after islet transplantation is necessary to keep the islets functional and alive, the LBL assembly system represents a new approach for improving vascularization. Therefore, we combining the LBL assembly system and the microwell scaffold the round morphology of the islets will be preserved by the microwell, and the vascularization will be improved witch may highly improve the outcome of islet transplantation.

\section{Outlook}

Based on the results of this research, we have identified several aspects of the islet microenvironment that will benefit future investigations. Pancreatic islets are highly vascularized multicellular micro-organs. The vessels around each islet provide the essential oxygen, nutrients, and hormones. Lack of vascularization results in depletion of nutrients and oxygen, which leads to starvation-induced cell death. Indeed, lack of vascularization has been reported as a main cause of islet loss following their transplantation. There are different strategies to improve 
vascularization either by stimulating revascularization or prevascularization of the scaffold [23-26]. Stimulation of revascularization using different growth factors such as VEGF has been reported as an efficient method. In chapter 7 we indicated an efficient layer by layer assembling method for the controlled release of growth factors. This system can be used for functionalization of our scaffold to accelerate revascularization after transplantation (in vivo).

In vitro previsualization by pre-culturing of endothelial cells on the scaffold could accelerate vessel formation immediately after implantation in vivo. In other studies, it was reported that co-culturing of various cell types such as bone marrow progenitor cells, endothelial cells, MSCs and fibroblast with endothelial cells potential could enhance the rate of vascularization. It is reported that co-culturing endothelial cells with support cells such as fibroblasts and MSCs could significantly enhance the efficiency of revascularization. Smink et al. indicated that in vivo vascularization of the scaffolds before transplantation of the islets has a positive effect on the viability and function of the islets [23]. Otherwise prevascularization of the microwell scaffold may improve further islets function. It is hypothesized that a layer of a highly hydrophobic polymer such as polyethylene located at the inside of the microwell scaffold may induce sufficient vascularization of the scaffold upon implantation in vivo for a few weeks. Then, the hydrophobic material will be removed and the space created will be used for islet transplantation in the prevascularized scaffold. Other techiques may also be investigated. As an example, it was demonstrated by Johansson et al., that incubation of endogenous human islets in prolactin prior to transplantation in the renal capsule of C57BL/6 mice enhanced revascularization as well as improved blood perfusion and oxygen tension [27].

The microwell scaffold appears efficient as an immune protective device. Loudovaris et al. indicated that a $0.45 \mu \mathrm{m}$ pore size membrane can successfully shield the allogenic cells from immunogenic reactions [28]. Encapsulation of islets in a scaffold with a $5 \mu \mathrm{m}$ pore size allows infiltration of IgG, IgM and formation of a capillary network into the scaffold while the allogenic tissue survived. Decreasing 
the pore size of the microwells from $40 \mu \mathrm{m}$ to $5 \mu \mathrm{m}$ in combination with an increase of porosity of the microwell scaffold may be regarded a potential solution.

The low availability as well as suboptimal quality of isolated islets from donors contributes to the low long-term success of islet transplantation. Therefore, an alternative cell source for islet transplantation can increase the efficiency of this method for treatment of type 1 diabetes. Application of porcine islets, human embryonic stem cells, human induced pluripotent stem cell derived pancreatic progenitor cells were reported as an insulin producing cell source for transplantation. The microwell scaffold may be applicable not only for human islets but also for alternative cell sources [29, 30]. As presented in chapter 4 and 5, we introduced an efficient method for enhancement of the function of $\beta$-cells by using ECM molecules and a controllable cluster size using microcontact printing. This technology allowed the generation of islet like structures with high functionality. Generation of $\beta$-cells from alternative sources, such as stem cells, on microcontact printed spots of ECM molecules inside each well of the scaffold may increase the efficiency of the microwells and needs further investigation.

In summary, in this research we provided key factors by mimicking the native microenvironment of islets which can be used for engineering of a definite islet transplantation technique and improving the outcome of islet transplantation. 


\section{References}

1. Schoonover, J.R. et al. (2001) Infrared linear dichroism study of a hydrolytically degraded poly (ester urethane). Polymer degradation and stability 74 (1), 87-96.

2. Stokes, K. et al. (1995) Polyurethane elastomer biostability. Journal of biomaterials applications 9 (4), 321-354.

3. Deschamps, A.A. et al. (2001) Poly (ethylene oxide)/poly (butylene terephthalate) segmented block copolymers: the effect of copolymer composition on physical properties and degradation behavior. Polymer 42 (23), 9335-9345.

4. Thivolet, C. et al. (1985) Morphological and functional effects of extracellular matrix on pancreatic islet cell cultures. Experimental cell research 159 (2), 313322.

5. Beattie, G.M. et al. (1996) Regulation of proliferation and differentiation of human fetal pancreatic islet cells by extracellular matrix, hepatocyte growth factor, and cell-cell contact. Diabetes 45 (9), 1223-1228.

6. Jiang, F.-X. et al. (1999) Laminin-1 promotes differentiation of fetal mouse pancreatic beta-cells. Diabetes 48 (4), 722-730.

7. Edamura, K. et al. (2003) BRIEF COMMUNICATION: Effect of Adhesion or Collagen Molecules on Cell Attachment, Insulin Secretion, and Glucose Responsiveness in the Cultured Adult Porcine Endocrine Pancreas: A Preliminary Study. Cell transplantation 12 (4), 439-446.

8. Deijnen, J. et al. (1994) Distribution of collagens type I, type III and type V in the pancreas of rat, dog, pig and man. Cell and tissue research 277 (1), 115-121.

9. Navarro-Alvarez, N. et al. (2008) Reestablishment of microenvironment is necessary to maintain in vitro and in vivo human islet function. Cell transplantation 17 (1-1), 111-119.

10. Weber, L.M. et al. (2008) Cell-matrix interactions improve $\beta$-cell survival and insulin secretion in three-dimensional culture. Tissue Engineering Part A 14 (12), 1959-1968.

11. Edmondson, R. et al. (2014) Three-dimensional cell culture systems and their applications in drug discovery and cell-based biosensors. Assay and drug development technologies 12 (4), 207-218.

12. Cirulli, V. et al. (2000) Expression and Function of av $\beta 3$ and av $\beta 5$ Integrins in the Developing Pancreas. The Journal of cell biology 150 (6), 1445-1460.

13. Hopcroft, D. et al. (1985) Structure-function relationships in pancreatic islets: support for intraislet modulation of insulin secretion. Endocrinology 117 (5), 20732080. 
14. Andersson, A. (1978) Isolated mouse pancreatic islets in culture: effects of serum and different culture media on the insulin production of the islets. Diabetologia 14 (6), 397-404.

15. Benninger, R.K. and Piston, D.W. (2014) Cellular communication and heterogeneity in pancreatic islet insulin secretion dynamics. Trends in Endocrinology \& Metabolism 25 (8), 399-406.

16. Lehmann, R. et al. (2007) Superiority of small islets in human islet transplantation. Diabetes 56 (3), 594-603.

17. Giuliani, M. et al. (2005) Central necrosis in isolated hypoxic human pancreatic islets: evidence for postisolation ischemia. Cell transplantation 14 (1), 67-76.

18. MacGregor, R. et al. (2006) Small rat islets are superior to large islets in in vitro function and in transplantation outcomes. American Journal of PhysiologyEndocrinology and Metabolism 290 (5), E771-E779.

19. Weinberg, N. et al. (2007) Lineage tracing evidence for in vitro dedifferentiation but rare proliferation of mouse pancreatic $\beta$-cells. Diabetes 56 (5), 1299-1304.

20. Fu, V., LAMININS AS A POTENTIAL ENHANCER OF BETA CELLS: PROLIFERATION AND SUBSEQUENT GENE EXPRESSION FOR THERAPEUTIC TREATMENT OF DIABETES MELLITUS, The University of Utah, 2017.

21. Jansson, L. and Carlsson, P.-O. (2002) Graft vascular function after transplantation of pancreatic islets. Diabetologia 45 (6), 749-763.

22. Sakata, N. et al. (2014) Strategy for clinical setting in intramuscular and subcutaneous islet transplantation. Diabetes/metabolism research and reviews 30 (1), 1-10.

23. Smink, A.M. et al. (2017) The Efficacy of a Prevascularized, Retrievable Poly (D, L,-lactide-co-[epsilon]-caprolactone) Subcutaneous Scaffold as Transplantation Site for Pancreatic Islets. Transplantation.

24. Pepper, A.R. et al. (2015) A prevascularized subcutaneous device-less site for islet and cellular transplantation. Nature biotechnology 33 (5), 518-523.

25. Balamurugan, A. et al. (2003) Bioartificial pancreas transplantation at prevascularized intermuscular space: effect of angiogenesis induction on islet survival. Pancreas 26 (3), 279-285.

26. Linn, T. et al. (2003) Angiogenic capacity of endothelial cells in islets of Langerhans. The FASEB journal 17 (8), 881-883.

27. Johansson, M. et al. (2008) Prolactin treatment improves engraftment and function of transplanted pancreatic islets. Endocrinology 150 (4), 1646-1653.

28. Loudovaris, T. et al., Destruction of xenografts but not allografts within cell impermeable membranes, Transplantation proceedings, 1992, pp. 2291-2292. 
29. D'Amour, K.A. et al. (2006) Production of pancreatic hormone-expressing endocrine cells from human embryonic stem cells. Nature biotechnology 24 (11), 1392.

30. Kroon, E. et al. (2008) Pancreatic endoderm derived from human embryonic stem cells generates glucose-responsive insulin-secreting cells in vivo. Nature biotechnology 26 (4), 443. 



\section{Summary}

Type 1 diabetes is an autoimmune disease in which the immune system destroys the insulin producing ß-cells of the pancreatic islets, which results in absolute insulin deficiency. Currently, intrahepatic islet transplantation is a promising treatment for type 1 diabetes, which may result in insulin independency of patients for several years. However, islet failure shortly after transplantation, insufficient insulin secretion and shortage of donors are major obstacles for this therapy. The main objective of the research described in this thesis was to improve the outcome of islet transplantation. By mimicking the islet's native microenvironment and exploring various bioengineering strategies, we have developed devices that could be applied for successful transplantation of islets.

To set the stages for this thesis, I) we have provided a comprehensive review on the biological, biochemical and biophysical characteristics of the islet microenvironment and the relation of these parameters with islets function and survival. II) We compared the physical and mechanical properties of biomaterials to be considered in the design of scaffolds. III) We explored the effect of microcontact printed extracelular matrix (ECM) molecules on the function and viability of INS-1E cells by providing a 3D biomimetic microenvironment. IV) We developed a microwell scaffold platform to not only act as a mechanical support and preserve the native morphology of islets, but also to maximize nutrient diffusion, while islet perfusion was kept at a minimum. Moreover, to mimic the islet's native microenvironment, microwell scaffolds were coated with different ECM molecules solely and pairwise at various ratios. V) We fabricated porous microwell scaffolds to not only prohibit islet fusion but also stimulate islet revascularization, which are essential factors for islets function and survival post transplantation. We also evaluated the biocompatibility and potential of our fabricated porous microwell scaffolds to induce normoglycemia in rat models. VI) Finally, we focused on one of the most important obstacles in islet transplantation, 
which is insufficient vascularization. Therefore, we designed and compared two different in vitro growth factor releasing systems to induce vascularization.

Altogether, in this thesis we have developed microwell scaffolds and identified several aspects of the islet microenvironment to improve the outcome of islet transplantation to treat type 1 diabetes. 


\section{Samenvatting}

Type 1 diabetes is een auto immuun ziekte waarbij het immuun systeem insuline producerende ß-cellen van de eilandjes van Langerhans afbreekt, wat resulteert in een absoluut insuline tekort. Op dit moment is transplantatie van donor eilandjes van Langerhans via infusie in de poortader in het vaatbed van lever een veelbelovende behandeling voor type 1 diabetes, welke kan resulteren in een insuline onafhankelijkheid van patiënten voor meerdere jaren. Echter, falen van eilandjes van Langerhans kort na transplantatie, een tekort aan insuline uitscheiding en een tekort aan donoren zijn grote obstakels voor dit soort behandelingen. Het hoofddoel van het onderzoek beschreven in dit proefschrift was het ontwikkelen van strategiën waarmee de de uitkomst van eilandjes transplantatie verbeterd kan worden. Door het nabootsen van de micro-omgeving van het eilandje en het onderzoeken van verschillende bio-engineering strategieën, hebben we een apparaat ontworpen welke kan worden gebruikt voor het succesvol transplanteren van donor eilandjes.

De volgende onderwerpen worden behandeld in dit proefschrift; I) Het proefschrift beging met grondig review op het gebied van de biologische, biochemische en biofysische eigenschappen van de micro-omgeving van de eilandjes van Langerhans en de relatie van deze parameters met het functioneren en overleven van getransplanteerde eilandjes. II) We hebben een vergelijking gemaakt van de fysieke en mechanische eigenschappen van twee biomaterialen die overwogen kunnen worden voor het ontwerp van een dragermateriaal voor eilandjes transplantatie, een zogenaamde scaffold. III) We onderzochten het effect van microcontact printed extracelulaire matrix (ECM) eiwitten op het gedrag en het vermogen om te reageren op een stijging in glucose spiegels van INS-1E cellen die ß-cel achtige eigenschappen hebben. Met deze techniek zijn we in staat om een meer realistische 3D biomimetische micro omgeving te engineren. IV) We hebben een microwell scaffold ontwikkeld die niet alleen als mechanische ondersteuning 
dient voor eilandjes transplantatie maar de eilandjes ook ondersteund in het behouden van hun oorspronkelijke morfologie. Daarnaast is de scaffold ontworpen omde diffusie van voedings- en afvalstoffen te maximaliseren. Om de oorspronkelijke omgeving van de eilandjes zo veel mogelijk te benaderen hebben we vervolgens de welletjes gecoat met verschillende combinaties van ECM eiwitten die van nature voorkomen in het eilandje. V) We hebben vervolgens poreuze microwell scaffolds gemaakt om enerzijds fusie van eilandjes te voorkomen, en anderzijds de hun revascularisatie te stimuleren, hetgeen essentiële factoren zijn voor het functioneren en het overleven van eilandjes na een transplantatie. We hebben ook de biocompatibiliteit en de potentie van de door ons geproduceerde poreuze microwell scaffolds om normoglycemia in rat modellen te veroorzaken geëvalueerd. VI) Tot slot hebben wij ons gericht op een van de meest belangrijke obstakels van voor succesvolle eilandjes transplantatie, namelijk onvoldoende vascularisatie. We hebben dit gedaan door strategiën uit te testen voor de afgifte van groeifactoren die bloedvatvorming stimuleren.

Samengevat, in dit proefschrift hebben we microwell scaffolds ontwikkeld en verschillende aspecten van de micro-omgeving van eilandjes geïdentificeerd waarmee in de toekomst de uitkomst van de transplantatie van eilandjes van Langerhans voor de behandeling van type I diabetesverbeterd kan worden. 


\section{Acknowledgments}

The wonderful journey of $\mathrm{PhD}$ with all exciting challenges passed so fast. As this thesis is the output of unyielding support and encouragements of colleagues, friends and family, I would like to express my sincere appreciation and respect to all involved.

Firstly, I would like to express my sincere gratitude to my supervisor Prof. Marcel Karperinen, and my co-supervisor Dr. Aart van Apeldoorn for giving me opportunity to pursue my PhD project in the Department of Developmental Bioengineering. I have learnt many things from you, which not only have been useful for me over these years but also will be great lessons for my future goals and plans.

I also would like to thank my committee members Prof. Robert Passier, Prof. Dirk Grijpma, Prof. Dimitrios Stamatialis, Prof. Paul de Vos, Prof. Ruud Bank, Dr. Roman Truckenmüller and Prof. Hans Hilgenkamp. During my PhD, I was consistently inspired by your scientific researches. Your presence in my $\mathrm{PhD}$ defense ceremony will surely be a great honor for me.

I also would like to express my heartfelt thanks to Prof. Piet Dijkstra for all his continuous support and valuable inputs on my project, over these years. Your office was always open to have scientific discussions. Meeting with you has always given me super positive energy to overcome the challenges of my $\mathrm{PhD}$.

I would like to extend my sincere appreciation to Dr. Jeroen Leijten for all his support and valuable scientific feedbacks. Indeed, I learnt many treasured things from you in a short time. Your brilliant and creative mind, charismatic characteristic, integrity, discipline and passion for science have always inspired me to become an even better version of myself. I am so proud to have the opportunity of working with you. 
I would like to express my special thanks to Prof. Paul de Vos. Collaborating with you and your wonderful group to perform in vivo study in the University Medical Center Groningen (UMCG) was one of the best events of my PhD. Beside of you deep knowledge in diabetes, I could learn a lot from your unique management skill. I also would like to thank Sandra, Baart and Arjen for all their help. Working with you was my great pleasure.

I would also like to thank Prof. Pascal Jonkheijm. Collaborating with you and working in your department with a very friendly atmosphere was a great honor for me.

I would like to thank Prof. Lorenzo Moroni. It was my great honor to begin a collaboration with you. Your quick and detailed replies to my emails (24/7) along with your scientific feedbacks with strong and wide background in the field of tissue engineering have always inspired me. I would also like to thank my great friend, Febriyani Damanik. Collaboration with you on development of a self-assembled layer by layer (LBL) system was a great pleasure for me.

Dear paranymphs; Marzieh and Febriyani thanks for standing always behind of me. Spending time with you always cheers me up and gives me energy to overcome all the challenges of life. So grateful to have you in my life. You are the best.

I also thank my colleagues at the department of Developmental Bioengineering (DBE) for all the stimulating discussions, help, lunches and group outings. I express my special thanks to Janine, Ingrid, Tom, Vasilis, Kannan, Sakshi, Elaheh, Sarah, Jan, Joao, Bram, Lissana, Brenda, Yao, Corina, Parthiban, Leilei, Xiaobin, Rong, Jacqueline, Tim, Janneke, Shuan, Dirk-jan, Don, Lorenzo, Barbara, Jere, David, Michele, Paula, Jacqueline and Anne.

I would also like to expend my special thanks to my colleagues and friends from different departments at university of Twente for great scientific discussions and 
supports over these years. My special thanks to Dr. Christiaan Bruinink, Dr. Ruchi Bansal, Dr. Harry Blom, Andries, Jenny, Yusuf, Aisen, Jean-Babtis, Kasia, Adithya, Rolf, Bastien, Dooli, Nattali, Jenny, Jasper, Gulistan, Alireza, Sina, Damoon, Hossein, Frederik, Hasib, Faraz, Sarah, Renee, Ehsan.

My dear friends Ivan, David, Angad and Emmanuella, our trip to Maastricht and attending in the advance microscopy course was one the memorable courses during my PhD. Dariush (P.Kh), many thanks for your positive energy and support. It was always enjoyable for me to discuss about politics with you during lunch. David thanks for helping me to improve my Dutch language. Alireza, my best friend for the doing amazing adrenaline-rush activities. I will never forget the day that we were heading to the flight course in an orange-code weather. Dr. Farhad Motae, Babak, Parsa and Masoud many thanks for your support. Jeroen, special thanks for helping me to improve my Dutch language and teaching me jive and your support. Your curiosity about the details of my project always impressed me. Niloofer, Febriyani and Ziryan still missing the memorable days that we had all together at UT. Class Willem, my tallest friend ever, your creative mind and scientific achievements always inspire me. Wishing you all the best.

I would like to express my appreciation to Bettie for providing an opportunity to assist in taking care of animal facility of the university Twente. Indeed, it was great experience. I learnt a lot from you.

I would like to thank my master and bachelor students Nadia, Thijs and Bouke for joining me in this wonderful journey of $\mathrm{PhD}$.

My special thanks to my besties, Zainab and Fatemeh for all your support and positive energy form far distance.

I would like to express my special thanks to all my dear friends in the board of $\mathrm{PhD}$ Network of the University of Twente (P-NUT). Thanks to Sarah, Koen, Guido, Federica, Bugra, Koen, Effie, Ines, Matteo, Imke, Anvar, Ozlem, Francesco, Davood, 
Qianyu, Simone and Jan. All brainstorming meetings and activities with you for organizing the social activities and events for PhD students have been enjoyable for me.

Last but not the least, I would like to express a deep sense of gratitude to my family. My lovely parents, Azim and Ziba, my deepest gratitude and love for all of your dedication and limitless support and inspiration to follow my dreams. Your positive energies even from far distance, from Iran, always gave me power to take my steps stronger than before! You have always impressed me by precisely following my research and giving me new ideas. Indeed, words are powerless to express my appreciation to you.

My dearest brother, Rashed, thanks a million for all your kindness and continuous support and fruitful advices. You taught me that risk management and backup plan are essential to achieve success in all the step of life. Your wise points always have been useful for me.

My admirable sister, Darya, you came to the Netherlands in the third year of my $\mathrm{PhD}$ and your presence made my life sweeter than ever. You are not only my sister but also my best friend. Spending every single moment with you, the trips all over the Europe, fun plans, events and deep scientific discussions always have been absolutely delightful for me. 


\section{List of Publications}

\section{Peer-reviewed papers}

- In preparation

E. Hadavi, M Karperien, A.A. van Apeldoorn, J. Leijten Tissue engineering of Islets for treatment of type one diabetes.

E. Hadavi, P.J. Dijkstra, A.A. van Apeldoorn, M. Karperien. Degradation kinetics and changes of poly(ester urethane) and PEOT-PBT thin films used for internal biomedical applications.

E. Hadavi, J. Leijten, P.J. Dijkstra, P. Jonkheijm, A.A. van Apeldoorn, M. Karperien. Microcontact printing of extracellular matrix induces self-assemply of INS-1E cells in islet-like organoids with increased insulin secreation.

E. Hadavi, J. Leijten, P. Jonkheijm, P.J. Dijkstra, M. Engelse, E. de Koning, A.A. van Apeldoorn, M. Karperien. Mimicking the islet extra cellular matrix niche in microwell islet delivery device using collgen-IV and laminin-111 leads to improved insulin secretion in human islets.

E. Hadavi, F.F.R. Damanik, C.M. Bruinik, J. Leijten, P.J. Dijkstra, L. Moroni,, A.A. van Apeldoorn, M. Karperien Endothelial growth factor delivery system from assembled polymer nanolayers.

E. Hadavi, A.M.Smink, B.J. de Haan, R. de Vries, J. Leijten, A.A. van Apeldoorn, M Karperien, Paul de Vos. PEOT-PBT and poly(ester urethane) microwell scaffolds for extrahepatic islet transplantation (invivo).

E. Hadavi, A.M. Smink, P. de Vos, B.J. de Haan, R. de Vries, J. Leijten, P.J. Dijkstra, A.A. van Apeldoorn, M. Karperien. PEOT-PBT and poly(ester urethane) microwell scaffold for extrahepatic islet transplantation. 


\section{Conferances}

E. Hadavi, E.J.P de Koning, H.B.J. Karperien, P. Jonkheijm, A.A. van Apeldoorn, (2015) Stimulation of beta-cell function by combinatorial extracellular matrix proteins. Poster presentation in TERMIS, Boston MA, USA, 2015.

E. Hadavi, E.J.P. de Koning, H.B.J. Karperien, P. Jonkheijm, A.A. van Apeldoorn, Microcontact printing of extracellular matrix induces self-assemply of INS-1E cells in islet-like organoids with increased insulin secreation. Poster presentation in the Netherlands Society for Biomaterial and Tissue Engineering annual meeting (NBTE), Lunteren, The Netherlands, 2015.

E. Hadavi, E.J.P. de Koning, H.B.J. Karperien, P. Jonkheijm, A.A. van Apeldoorn, Stimulation of $ß$-cell function by extracellular matrix proteins. Poster presentation in the Netherlands Society for Biomaterial and Tissue Engineering annual meeting (NBTE), Lunteren, The Netherlands, 2014.

E. Hadavi, H.B.J. Karperien, A.A. van Apeldoorn, P. Jonkheijm. Optimization of ßcell function and viability. Poster presentation in New Frontiers Symposium in Regenerative Medicine, Nijmegen, The Netherlands, 2014

E. Hadavi, H.B.J. Karperien, A.A. van Apeldoorn, P. Jonkheijm. Role of extracellular matrix on function and viability of $ß$-cell. Poster presentation in MIRA conference, University of Twente, Enschede, The Netherlands, 2014.

E.Hadavi, H.B.J. Karperien, A.A. van Apeldoorn, Biomaterials for islet transplantation, Poster presentation in The BMM/TeRM/DCTI Annual Meeting on Biomaterial and Regenerative medicine, Ermelo, The Netherlands, 2013.

E.Hadavi, H.B.J. Karperien, A.A. van Apeldoorn. Biomaterials for islet tissue engineering. Poster presentation in International symposium on vascular tissue engineering, Leiden, The Netherlands, 2013. 


\section{Biography}

Elahe Hadavi was born on $21^{\text {st }}$ September 1984 in Ardebil, Iran. She studied her B.Sc in the field of "Cellular and Molecular Biology-Microbiology" in Azad University of Tonekabon, Iran. Beside of her bachelor curiculum, she also performed different side

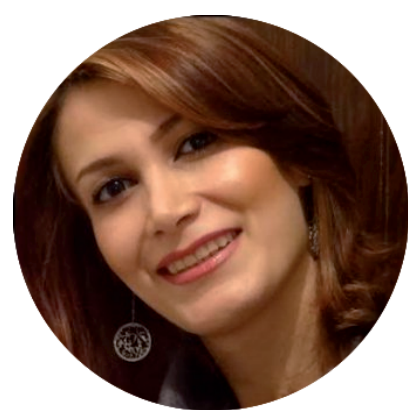
research projects and worked in various clinical laboratories as a technician of hematology, microbiology and biochemistry sections to enhance her practical experience. She accomplished her B.Sc. program cum laude (Top 2\%) in 2008.

After being awarded the highly competitive Nuffic Huygens Scholarship (HSP) (ranked 14 out of 3000 candidates), she began to study master of "Molecular Life Sciences" at University of Radboud, The Netherlands on August 2010. During her master's program she worked on two master's project of each 9 months. In her first year, she worked on a project entitled: "Identification of prostate cancer stem cells" under the supervision of Prof. Jack Schalken. In her second year of M.Sc. she worked on a project entitled: "Investigation of protective effect of heparin on FGF2 during gamma-sterilization for tissue engineering applications" under the supervision of Toin van Kuppevelt and Willeke Daamen. She successfully graduated her M.Sc on Aug 2012.

In 2013, she started her $\mathrm{PhD}$ at the Developmental BioEngineering within the MIRA Institute, University of Twente, under supervision of prof. Dr. Marcel Karperien and A.A. van Apeldoorn. Her project focused on "A Tissue Engineering Approach Towards Treatment of Type 1 Diabete". The results of her work are presented in this thesis. 




